\title{
Two cysteine-rich receptor-like protein kinases, CRK7 and CRK43, are required for CERK1-4 dependent cell death responses in Arabidopsis thaliana
}

\author{
Dissertation \\ zur Erlangung des mathematisch-naturwissenschaftlichen Doktorgrades \\ "Doctor rerum naturalium" \\ der Georg-August-Universität Göttingen \\ im Promotionsprogramm Biologie \\ der Georg-August University School of Science (GAUSS)
}

vorgelegt von

\section{Christine Trippel}

aus Schaafheim

Göttingen, 2020 

$\underline{\text { Betreuungsausschuss }}$

1. Betreuer: Prof. Dr. Volker Lipka

Zellbiologie der Pflanze

Albrecht-von-Haller Institut für Pflanzenwissenschaften

\section{Betreuer: PD Dr. Thomas Teichmann}

Zellbiologie der Pflanze

Albrecht-von-Haller Institut für Pflanzenwissenschaften

\section{Betreuer: PD Dr. Marcel Wiermer}

Molekularbiologie der Pflanze-Mikroben Interaktionen

Albrecht-von-Haller Institut für Pflanzenwissenschaften

\section{Anleiterin: Dr. Elena Petutschnig}

Zellbiologie der Pflanze

Albrecht-von-Haller Institut für Pflanzenwissenschaften

Mitglieder der Prüfungskommission

Referent: $\quad$ Prof. Dr. Volker Lipka

Zellbiologie der Pflanze

Albrecht-von-Haller Institut für Pflanzenwissenschaften

\section{Korreferent: PD Dr. Marcel Wiermer}

Molekularbiologie der Pflanze-Mikroben Interaktionen

Albrecht-von-Haller Institut für Pflanzenwissenschaften

$\underline{\text { Weitere Mitglieder der Prüfungskommission }}$

PD Dr. Thomas Teichmann

Zellbiologie der Pflanze

Albrecht-von-Haller Institut für Pflanzenwissenschaften

Prof. Dr. Kai Heimel

Mikrobielle Zellbiologie

Institut für Mikrobiologie und Genetik

Prof. Dr. Christiane Gatz

Molekularbiologie und Physiologie der Pflanze

Albrecht-von-Haller Institut für Pflanzenwissenschaften

Prof. Dr. Andrea Polle

Forstbotanik und Baumphysiologie

Fakultät für Forstwissenschaften und Waldökologie 


\section{Promovierenden-Erklärung der Georg-August-Universität Göttingen}

Die Gelegenheit zum vorliegenden Promotionsvorhaben ist mir nicht kommerziell vermittelt worden. Insbesondere habe ich keine Organisation eingeschaltet, die gegen Entgelt Betreuerinnen und Betreuer für die Anfertigung von Dissertationen sucht oder die mir obliegenden Pflichten hinsichtlich der Prüfungsleistungen für mich ganz oder teilweise erledigt.

Hilfe Dritter wurde bis jetzt und wird auch künftig nur in wissenschaftlich vertretbarem und prüfungsrechtlich zulässigem Ausmaß in Anspruch genommen. Insbesondere werden alle Teile der Dissertation selbst angefertigt; unzulässige fremde Hilfe habe ich dazu weder unentgeltlich noch entgeltlich entgegengenommen und werde dies auch zukünftig so halten.

Die Ordnung zur Sicherung der guten wissenschaftlichen Praxis an der Universität Göttingen wird von mir beachtet.

Eine entsprechende Promotion wurde an keiner anderen Hochschule im In- oder Ausland beantragt; die eingereichte Dissertation oder Teile von ihr wurden nicht für ein anderes Promotionsvorhaben verwendet.

Mir ist bekannt, dass unrichtige Angaben die Zulassung zur Promotion ausschließen bzw. später zum Verfahrensabbruch oder zur Rücknahme des erlangten Grades führen. 


\section{Abstract}

Plants recognize potential pathogens via conserved molecular patterns. These are perceived by plasma membrane-associated receptor complexes leading to downstream signaling and activation of the plant immune system. In Arabidopsis thaliana, the LysM containing kinase CERK1 is an essential part of the receptor for chitin, a major component of fungal cell walls. Besides full length CERK1, a shorter N-terminal fragment is present, that is generated by ectodomain shedding and released into the apoplast. This N-terminal fragment cannot be detected in plants carrying a CERK1 mutant version with an amino acid exchange in the ectodomain (CERK1-4). Furthermore, cerk1-4 mutants show a deregulated salicylic acid dependent cell death response upon infection with powdery mildews and in senescent plants, whereas chitin responses are WT-like. The role of the shed ectodomain and the mechanism underlying the deregulated cell death response are still unclear. To identify molecular components necessary for cell death induction in cerk1-4, a suppressor screen was conducted.

In this work, three independent suppressor mutations were identified. In one mutant line suppression of the cerk1-4 phenotype was probably caused by an amino acid exchange in PAD4. Since PAD4 was already known to be necessary for cerk1-4 cell death induction, this mutant was not further characterized. The other suppressor mutations affected two different cysteine-rich receptor-like protein kinases, CRK7 and CRK43. CRKs are one of the largest subgroups among Arabidopsis receptor-like kinases and contain characteristic and highly conserved cysteine-rich motifs in their ectodomains. Moreover, several CRKs were shown to play a role in plant immunity and cell death regulation. CRK7 and CRK43 were confirmed as components of the cerk1-4 pathway by independent mutants and/ or complementation approaches and localization studies were conducted. In both crk7 and crk43 mutants, immune responses upon chitin and flg22 treatment were not altered compared to the wildtype.

In a point mutant isolated from the cerk1-4 suppressor screen (CRK7-4), a highly conserved cysteine residue in the ectodomain is mutated. Co-immunoprecipitation analyses revealed that CRK7 is able to interact with itself whereas CRK7-4 is not. Moreover, it was shown that CRK7 localizes predominantly to the plasma membrane, while CRK7-4 is found in the ER. Structural comparisons with related proteins and proteomics data suggest that CRK7-4 is misfolded, resulting in ER retention. The phosphorylation status was investigated in CRK7 and CRK7-4 isolated from Arabidopsis or Nicotiana benthamiana plants. Strongly phosphorylated amino acids in the activation and $\mathrm{P}+1$ loop of the kinase domain were identified in CRK7, but phosphorylation of these residues was strongly reduced in CRK7-4. Nevertheless, in vitro kinase activity was shown for both CRK7 and 
CRK7-4. A mutant in which all phosphorylateable amino acids in the activation and $\mathrm{P}+1$ loop of CRK7 were exchanged for alanine was unable to autophosphorylate or to phosphorylate an artificial substrate. These findings suggest that CRK7 might be phosphorylated and activated after homomerisation. CRK7 might function as a receptor perceiving either the CERK1 ectodomain, turnover products of the CERK1-4 ectodomain, or another yet unknown signal, and subsequently mediate cell death induction in cerk1-4. 


\section{Zusammenfassung}

Pflanzen erkennen potentiell pathogene Mikroorganismen anhand konservierter molekularer Strukturen, welche von Plasmamembran-assoziierten Rezeptorkomplexen gebunden werden. Dies führt zur Signaltransduktion und zur Aktivierung des pflanzlichen Immunsystems. In Arabidopsis thaliana ist die LysM Rezeptorkinase CERK1 ein essentieller Bestandteil des Rezeptors für Chitin, einer Hauptkomponente der pilzlichen Zellwand. Zusätzlich zum CERK1-Volllängenprotein ist ein Ektodomänenfragment vorhanden, welches durch so genanntes Ektodomänen-Shedding von CERK1 abgespalten und in den Apoplasten freigesetzt wird. Bei einer mutierten Variante von CERK1, die einen Aminosäureaustausch in der Ektodomäne trägt (CERK1-4), kann das Nterminale Ektodomänenfragment nicht nachgewiesen werden. Des Weiteren zeigen cerk1-4 Pflanzen eine deregulierte Salizylsäure-abhängige Zelltodreaktion nach Infektion mit Mehltaupilzen und in seneszenten Pflanzen, weisen jedoch eine Wildtyp-ähnliche Chitinantwort auf. Bisher ist nicht bekannt, wie die deregulierte Zelltodreaktion induziert wird und welche Funktion die CERK1 Ektodomäne hat. Um molekulare Komponenten, die an der Ausbildung des Zelltods beteiligt sind, zu identifizieren, wurde ein cerk1-4 Suppressor-Screen durchgeführt.

In dieser Arbeit wurden Polymorphismen, die zur Suppression des Zelltod-Phänotyps führen, in drei unabhängigen Mutanten identifiziert. In einer Mutante war vermutlich ein Aminosäureaustausch in PAD4 für die Unterdrückung des Zelltods verantwortlich. Da jedoch bereits bekannt war, dass PAD4 für die Induktion des cerk1-4-abhängigen Zelltods notwendig ist, wurde dies nicht weiter verfolgt. In den beiden anderen isolierten Mutanten waren Mutationen in den Cysteinreichen Rezeptorkinasen CRK7 und CRK43 die Ursache des Suppressor-Phänotyps. CRKs stellen eine große Gruppe innerhalb der Rezeptorkinasen dar und zeichnen sich durch charakteristische Cystein-reiche Motive in der Ektodomäne aus. Für etliche CRKs wurde auch gezeigt, daß sie in der pflanzlichen Immunität und der Zelltod-Regulation eine Rolle spielen. Beide CRKs wurden mittels unabhänger Mutanten und/ oder Komplementationsanalysen als Komponenten der cerk1-4 Signaltransduktion bestätigt und hinsichtlich ihrer zellulären Lokalisation untersucht. Sowohl in den identifizierten $c r k 7$ als auch crk43 Mutanten waren die Immunantworten nach Chitin und flg22 Behandlung vergleichbar zum Wildtyp.

In der im Suppressor-Screen identifizierten Punktmutante crk7-4 ist ein hoch konserviertes Cystein in der Ektodomäne mutiert. Co-Immunoprezipitationsanalysen zeigten, dass CRK7 Homomere ausbildet, CRK7-4 jedoch nicht. Außerdem wurde gezeigt, dass CRK7 vorrangig an der Plasmamembran und CRK7-4 im ER lokalisiert. Vergleiche mit verwandten Proteinen sowie ProteomicsDaten legen nahe, dass CRK7-4 durch die Mutation des konservierten Cysteins falsch gefaltet vor- 
liegt und daher im ER festgehalten wird. CRK7 und CRK7-4 wurden aus Arabidopsis oder Nicotiana benthamiana isoliert und hinsichtlich phosphorylierter Aminosäuren untersucht. Dabei wurden stark phosphorylierte Aminosäuren im Aktivierungs- und P+1-Loop von CRK7 identifiziert, diese zeigten jedoch eine deutlich reduzierte Phosphorylierung in CRK7-4. Dennoch wurde in vitro Kinase-Aktivität sowohl für CRK7 als auch für CRK7-4 nachgewiesen. Eine Mutante, in welcher alle phosphorylierbaren Aminosäuren im Aktivierungs- und P+1-Loop gegen Alanine ausgetauscht wurden, war jedoch nicht in der Lage sich selbst oder ein artifizielles Substrat zu phosphorylieren. Dies legt nahe, daß CRK7 durch Homomerisierung phosphoryliert und aktiviert wird. CRK7 könnte möglicherweise als Plasmamembran-Rezeptor für die CERK1 Ektodomäne, CERK1-4 Ektodomänen-Fragmente oder ein anderes bisher unbekanntes Signal fungieren und daraufhin Zelltod auslösen. 


\section{List of abbreviations}

\begin{tabular}{|c|c|c|c|}
\hline$\alpha$ & anti/ alpha & CRISPR & clustered regularly interspaced \\
\hline$\Omega$ & $\mathrm{Ohm}$ & & short palindromic repeats \\
\hline${ }^{\circ} \mathrm{C}$ & degree Celsius & CRK & CYSTEINE-RICH RECEPTOR- \\
\hline$\mu \mathrm{g}$ & microgramm & & LIKE PROTEIN KINASE \\
\hline$\mu 1$ & microliter & CRRSP & CYSTEINE-RICH RECEPTOR- \\
\hline$\mu \mathrm{m}$ & micrometer & & LIKE SECRETED PROTEIN \\
\hline$\mu \mathrm{M}$ & micromolar & $\mathrm{Da}$ & dalton \\
\hline ABA & abscisic acid & DAMP & damage-associated \\
\hline AFP1/2 & ANTIFUNGAL PROTEIN $1 / 2$ & & pattern \\
\hline AP & alkaline phosphatase & $\mathrm{ddH}_{2} \mathrm{O}$ & double-distilled water \\
\hline APS & ammonium persulfate & DMSO & dimethylsulfoxid \\
\hline$A t$ & Arabidopsis thaliana & DNA & desoxyribonucleic acid \\
\hline Avr & avirulence & dpi & day post infection/ infiltration \\
\hline BAK1 & BRI1-ASSOCIATED KINASE 1 & DTT & dithiothreitol \\
\hline$B g h$ & Blumeria graminis f.sp. hordei & DUF26 & domain of unknown function 26 \\
\hline \multirow[t]{2}{*}{ BIK1 } & BOTRYTIS-INDUCED KINASE & DYT & double yeast tryptone \\
\hline & 1 & $E c$ & Erysiphe cruciferarum \\
\hline BKK1 & BAK1-LIKE 1 & EDS1 & ENHANCED DISEASE SUS- \\
\hline $\mathrm{bp} / \mathrm{bps}$ & base pair/ base pairs & & CEPTIBILITY 1 \\
\hline $\mathrm{Bq}$ & becquerel & EDTA & ethylenediaminetetraacetic acid \\
\hline $\mathrm{BR}$ & brassinosteroid & EFR & elongation-factor thermo unstable \\
\hline \multirow[t]{2}{*}{ BRI1 } & BRASSINOSTEROID INSEN- & & receptor \\
\hline & SITIVE 1 & EMS & ethyl methane sulfonate \\
\hline BSA & bovine serum albumin & ER & endoplasmic reticulum \\
\hline CaMV & Cauliflower mosaic virus & et al. & et alii; and others \\
\hline $\mathrm{CC}$ & coiled-coil & ETI & effector-triggered immunity \\
\hline cDNA & complementary DNA & ETS & effector-triggered susceptibility \\
\hline \multirow[t]{2}{*}{ CEBiP } & CHITIN ELICITOR BINDING & f.sp. & forma specialis \\
\hline & PROTEIN & $\mathrm{F} 1 / 2$ & filialgeneration $1 / 2$ \\
\hline \multirow[t]{2}{*}{ CERK1 } & CHITIN ELICITOR RECEPTOR & flg22 & flagellin ( 22 amino acid peptide) \\
\hline & KINASE & FLS2 & FLAGELLIN SENSING 2 \\
\hline CNL & CC-NLR & $\mathrm{g}$ & gram or gravitation \\
\hline \multirow[t]{3}{*}{ CRCK } & CYSTEINE-RICH RECEPTOR- & GFP & green fluorescence protein \\
\hline & LIKE CYTOPLASMIC KINASE & GlcNAc & N-acetyl-D-glucosamine \\
\hline & & GNK2 & GINKBILOBIN 2 \\
\hline
\end{tabular}




\begin{tabular}{|c|c|c|c|}
\hline Go & Golovinomyces orontii & MBP & myelin basic protein \\
\hline GPI-anchor & $\begin{array}{l}\text { glycosylphosphatidylinositol- } \\
\text { anchor }\end{array}$ & MES & $\begin{array}{l}\text { 2-(N-morpholino)ethanesulfonic } \\
\text { acid }\end{array}$ \\
\hline gRNA & guide RNA & $\min$ & minute \\
\hline GST & gluthathione-S-transferase & $\mathrm{ml}$ & milliliter \\
\hline $\mathrm{h}$ & hour(s) & $\mathrm{mm}$ & millimeter \\
\hline Hpa & Hyaloperanospora arabidopsidis & $\mathrm{mM}$ & millimolar \\
\hline HR & hypersensitive response & MOPS & 3-(N-morpholino)propanesulfonic \\
\hline HRP & horseradish peroxidase & & acid \\
\hline HrpZ & harpinZ & MOS7 & MODIFIER OF SNC1 7 \\
\hline i.e. & id est & MS & Murashige-Skoog \\
\hline IAM & iodoacetamide & $M t$ & Medicargo truncatula \\
\hline ICS1 & $\begin{array}{l}\text { ISOCHORISMATE SYNTHASE } \\
1\end{array}$ & NADPH & $\begin{array}{l}\text { nicotinamidadenindinukleotid } \\
\text { phosphat }\end{array}$ \\
\hline IP & immunoprecipitation & NASC & Nottingham Arabidopsis Stock \\
\hline JX & Juxtamembrane domain & & Center \\
\hline $\mathrm{kDa}$ & kilo Dalton & $\mathrm{Nb}$ & Nicotiana benthamiana \\
\hline 1 & liter & NB & nucleotide binding \\
\hline LB & $\begin{array}{l}\text { Lysogeny broth/ Luria-Bertani } \\
\text { broth or left border primer }\end{array}$ & NDR1 & $\begin{array}{l}\text { NON-RACE-SPECIFIC } \\
\text { EASE RESISTANCE } 1\end{array}$ \\
\hline$L j$ & Lotus japonicus & nESI & nano-electrospray \\
\hline LRR & leucine-rich repeat & NF & nodulation factor \\
\hline LYK & LYSM RECEPTOR-LIKE KI- & NFP & NOD FACTOR PERCEPTION \\
\hline & NASE & NFR & NOD FACTOR RECEPTOR \\
\hline LYM & LYSM RECEPTOR-LIKE PRO- & NGS & next generation sequencing \\
\hline & TEIN & NHR & non-host resistance \\
\hline LYP & LYSM CONTAINING PRO- & NiCK4 & NFR5-INTERACTING CYTO- \\
\hline & TEIN & & PLASMIC KINASE 4 \\
\hline LYR3 & $\begin{array}{l}\text { LysM RECEPTOR-LIKE KI- } \\
\text { NASE } 3\end{array}$ & NLR & $\begin{array}{l}\text { nucleotide-binding leucine-rich } \\
\text { repeat }\end{array}$ \\
\hline LysM & lysin motif & $\mathrm{nm}$ & nanometer \\
\hline M & molar & $\mathrm{nM}$ & nanomolar \\
\hline $\mathrm{m}$ & meter & NNP1 & NECROSIS-INDUCING \\
\hline $\mathrm{M} 1 / 2 / 3$ & mutagenesis generation $1 / 2 / 3$ & & TOPHTORA PROTEIN 1 \\
\hline MAMP & microbe associated molecular & noce & no cerk1-4 cell death phenotype \\
\hline & pattern & Nod & Nodulation \\
\hline
\end{tabular}

MAPK mitogen-activated protein kinase 


\begin{tabular}{|c|c|c|c|}
\hline \multirow[t]{2}{*}{ NOD } & Nucleotide binding oligomeriza- & RLP & receptor-like protein \\
\hline & tion domain & RNA & ribonucleic acid \\
\hline \multirow[t]{3}{*}{ NPR1 } & NON-EXPRESSOR OF PATH- & ROS & reactive oxygen species \\
\hline & OGENESIS-RELATED GENES & $\mathrm{rpm}$ & rounds per minute \\
\hline & 1 & RT & room temperature \\
\hline NUP88 & NUCLEOPORIN 88 & $\mathrm{~s}$ & second(s) \\
\hline OD & optical density & SA & salicylic acid \\
\hline $\mathrm{OE}$ & overexpression & SAG101 & SENESCENCE-ASSOCIATED \\
\hline Os & Oryza sativa & & GENE 101 \\
\hline \multirow[t]{2}{*}{ OSER } & organized smooth endoplasmic & SAP & shrimp alkaline phosphatase \\
\hline & reticulum & SAR & systemic acquired resistance \\
\hline PAD4 & PHYTOALEXIN-DEFICIENT 4 & SDS & sodium dodecyl sulfate \\
\hline PAM & protospacer adjacent motif & SDS-PAGE & sodium dodecyl sulfate poly- \\
\hline \multirow[t]{2}{*}{ PAMP } & pathogen-associated molecular & & acrylamide gel electrophoresis \\
\hline & pattern & SERK & SOMATIC-EMBRYOGENESIS \\
\hline PCR & polymerase chain reaction & & RECEPTOR-LIKE KINASE \\
\hline $\mathrm{PD}$ & plasmodesma(ta) & SID2 & SA-DEFICIENT MUTANT 2 \\
\hline \multirow[t]{2}{*}{ PDLP } & PLASMODESMATA LOCAL- & SNP & single nucleotide polymorphism \\
\hline & IZED PROTEIN & SOBIR1 & SUPPRESSOR OF BIR1-1 \\
\hline PEP1 & PLANT ELICITOR PROTEIN 1 & SYMRK & SYMBIOSIS RECEPTOR-LIKE \\
\hline PEPR & PEP receptor & & KINASE \\
\hline pFAM & protein families & $\mathrm{T} 1 / 2$ & transformed generation $1 / 2$ \\
\hline PGN & peptidoglycan & TAE & Tris-acetic acid EDTA \\
\hline PM & plasma membrane & TAIR & The Arabidopsis Information \\
\hline PR & PATHOGENESIS RELATED & & Resource \\
\hline PRR & pattern recognition receptor & TBS-T & tris buffered saline - Tween- 20 \\
\hline \multirow[t]{2}{*}{ Pst } & Pseudomonas syringae pv. toma- & T-DNA & transfer-DNA \\
\hline & to & TEMED & tetramethylethylenediamine \\
\hline PTI & pattern-triggered immunity & TFA & trifluoroacetic acid \\
\hline pv. & pathovar & TIR & toll-Interleukin-1 receptor \\
\hline PVDF & polyvinylidene fluoride & TNL & TIR-NLR \\
\hline $\mathrm{R}$ & resistance & TTSS & type III secretion system \\
\hline \multirow[t]{2}{*}{ RBOHD/F } & RESPIRATORY BURST OXI- & $\mathrm{U}$ & unit \\
\hline & DASE HOMOLOG D/F & UTR & untranslated region \\
\hline rel. & relative & UV & ultraviolet \\
\hline RLCK & receptor-like cytoplasmic kinase & $\mathrm{V}$ & volt \\
\hline RLK & receptor-like kinase & & \\
\hline
\end{tabular}


WRKY transcription factor with WRKY

amino acid sequence at the $\mathrm{N}$ -

terminus

WT

wild type

XLG2

EXTRA-LARGE G-PROTEIN

ZAR1

HOPZ-ACTIVATED RESIS-

TANCE 1 


\section{Table of contents}

ABSTRACT

ZUSAMMENFASSUNG .III

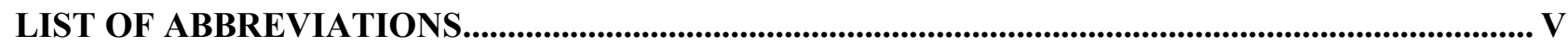

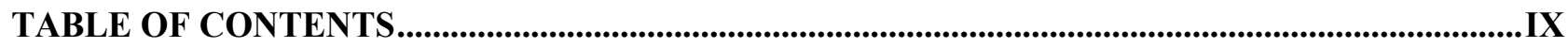

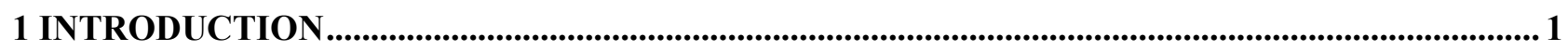

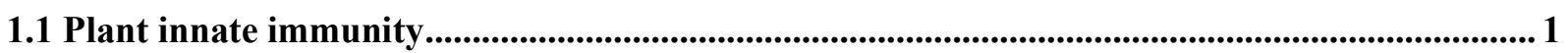

1.2 MAMP recognition via pattern recognition receptors.................................................................5

1.2.1 LRR-RLK complexes and the recognition of peptide MAMPs ................................................ 5

1.2.2 LysM-RLK complexes and the recognition of carbohydrate MAMPs ...................................... 7

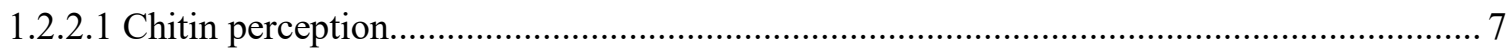

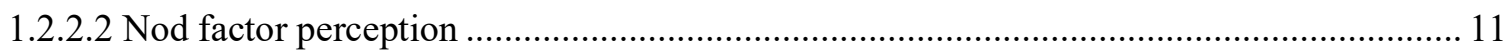

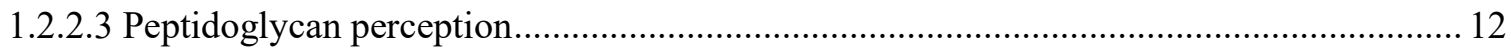

1.3 A CERK1 mutant with enhanced pathogen-induced cell death and altered receptor processing .............................................................................................................................................................. 13

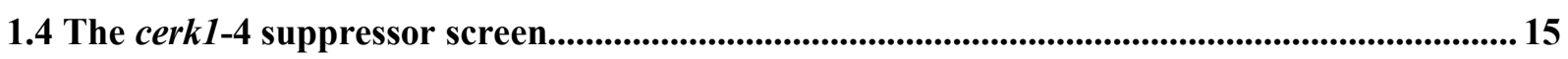

1.5 DUF26 domain containing proteins in plants ................................................................................. 16

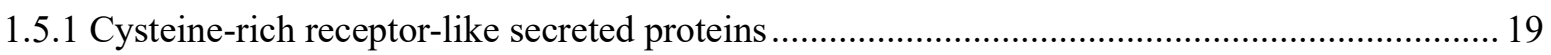

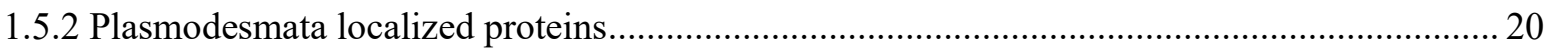

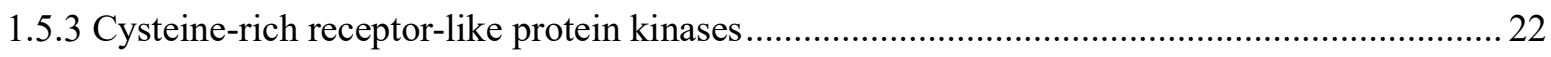

1.6 Thesis aims ...................................................................................................................................................... 30

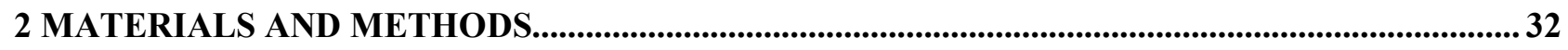

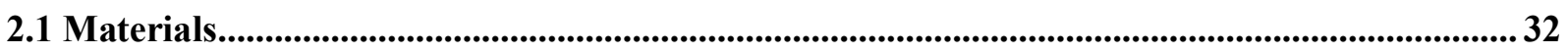

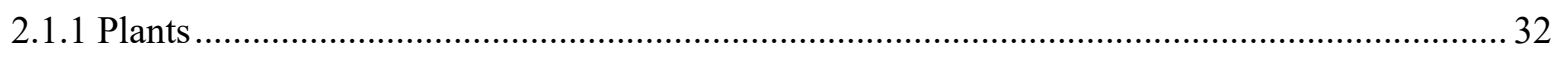

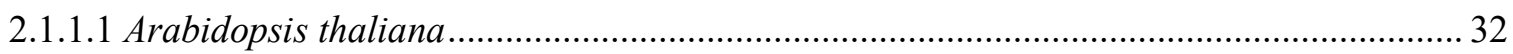

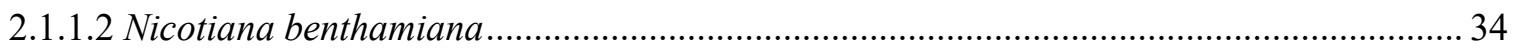

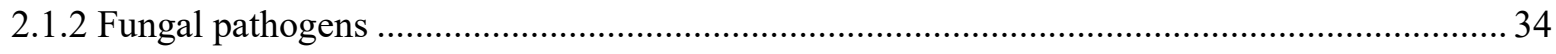

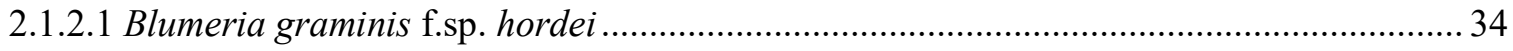

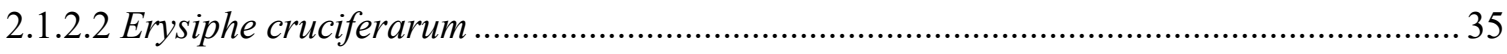

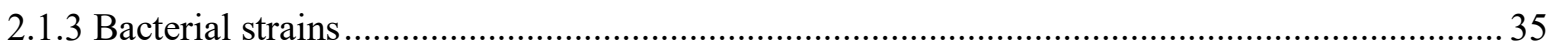

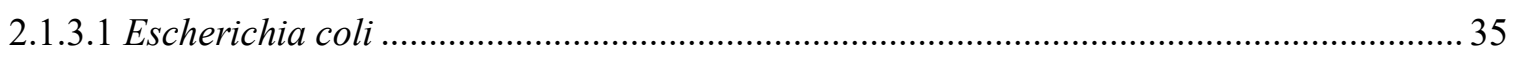

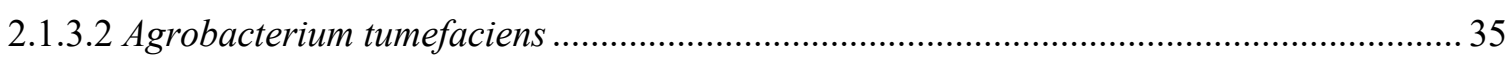

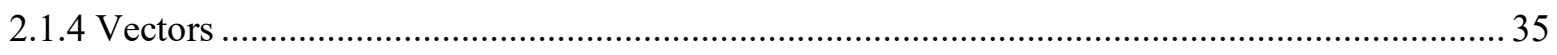

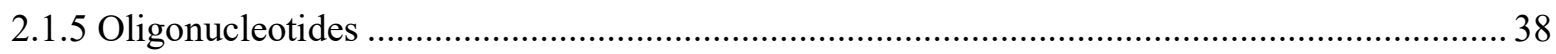




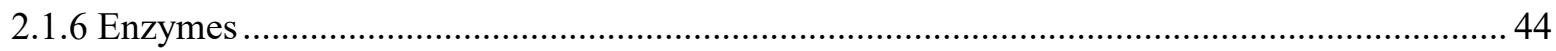

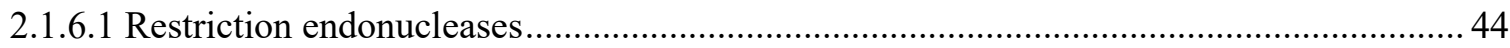

2.1.6.2 Polymerases and nucleic acid modifying enzymes........................................................... 44

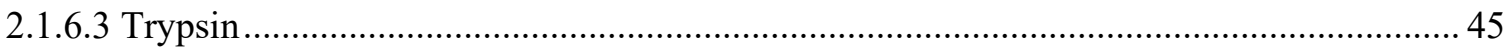

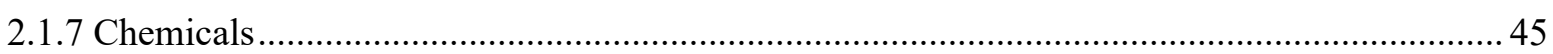

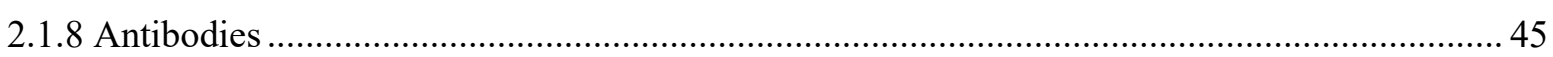

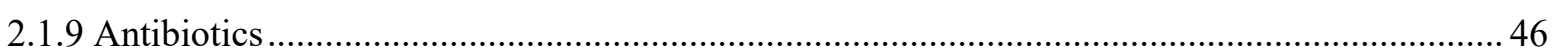

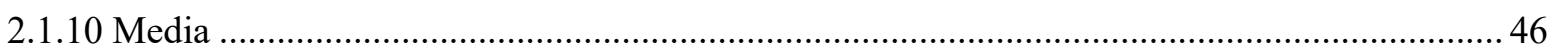

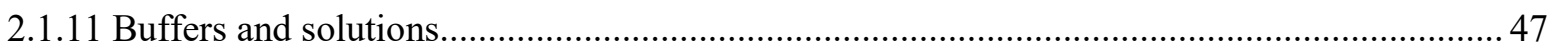

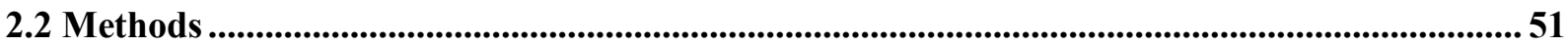

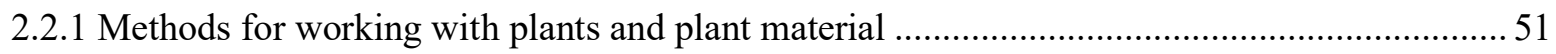

2.2.1.1 Methods for working with Arabidopsis thaliana ............................................................... 51

2.2.1.1.1 Surface sterilization of Arabidopsis seeds................................................................. 51

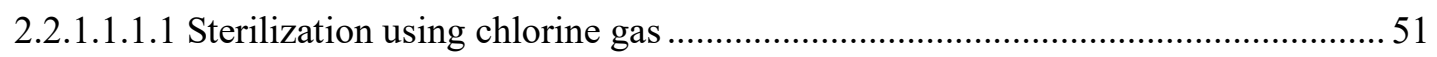

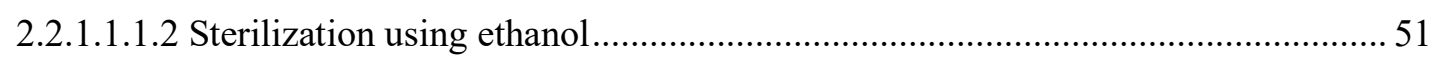

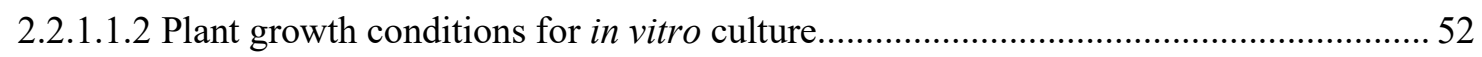

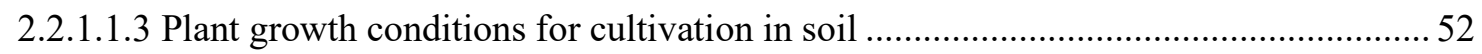

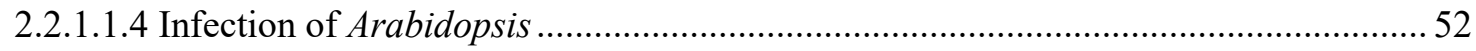

2.2.1.1.4.1 Infection with Blumeria graminis f. sp. hordei (Bgh) ....................................... 52

2.2.1.1.4.2 Infection with Erysiphe cruciferarum ............................................................. 53

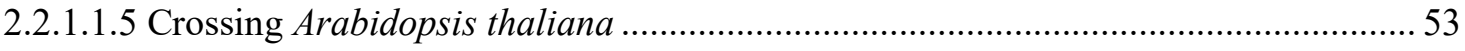

2.2.1.1.6 Agrobacterium-mediated stable transformation of Arabidopsis................................. 53

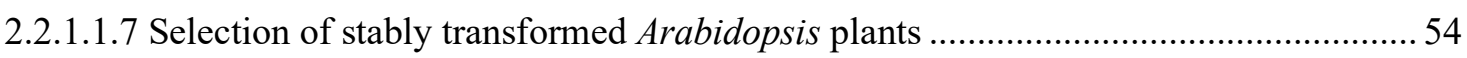

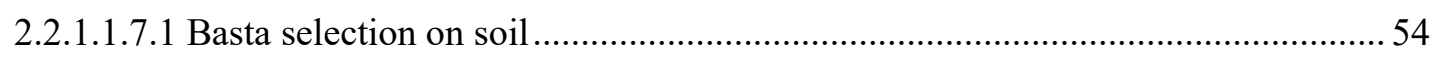

2.2.1.1.7.2 In vitro selection of Arabidopsis transformands ................................................... 54

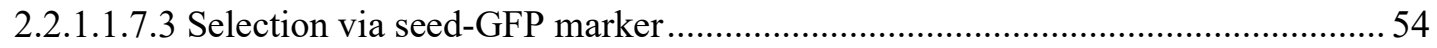

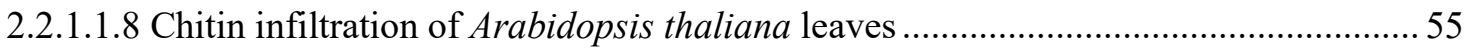

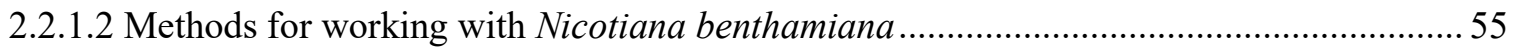

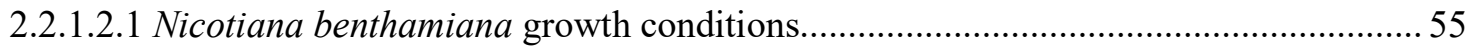

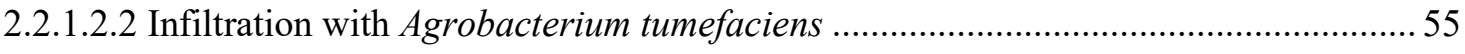

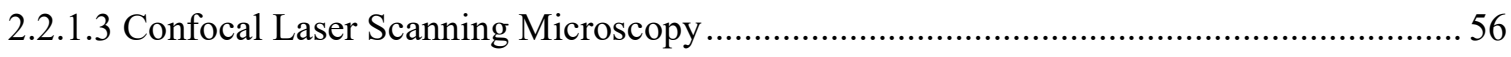

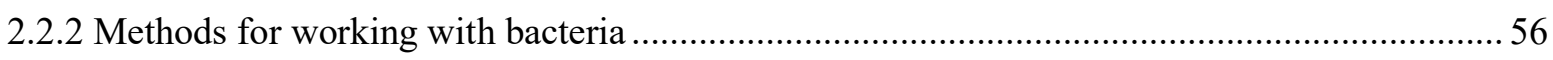

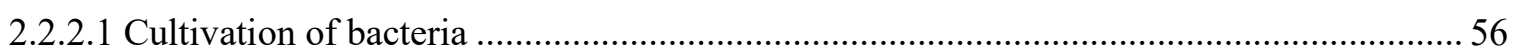

2.2.2.2 Preparation of chemically competent E.coli cells ............................................................ 57

2.2.2.3 Preparation of electro-competent $A$. tumefaciens cells .................................................... 57

2.2.2.4 Transformation of chemically competent E.coli cells .................................................... 57

2.2.2.5 Transformation of electro-competent $A$. tumefaciens cells.............................................. 58 


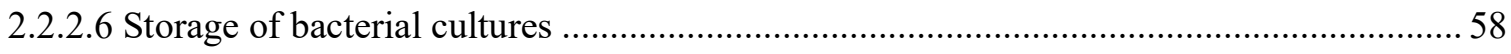

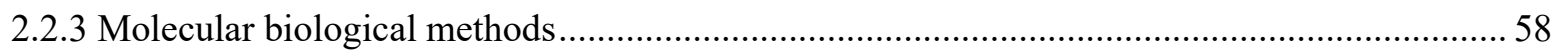

2.2.3.1 Isolation of genomic DNA (gDNA) from Arabidopsis thaliana ..........................................58

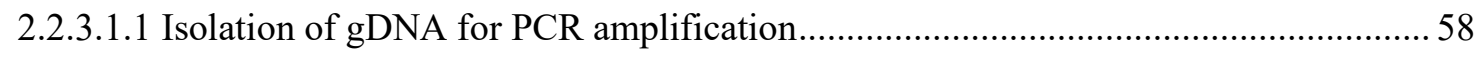

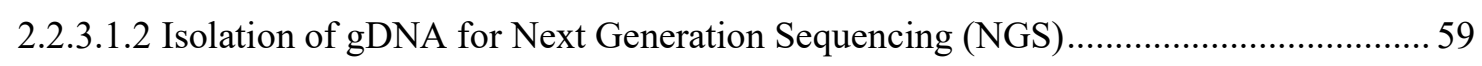

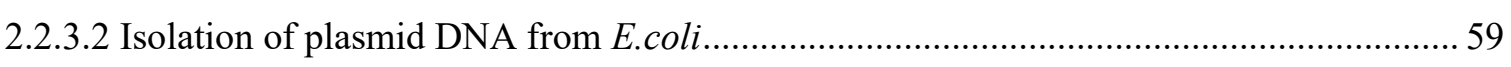

2.2.3.2.1 Small-scale plasmid preparation (Birnboim and Doly, 1979) ................................... 59

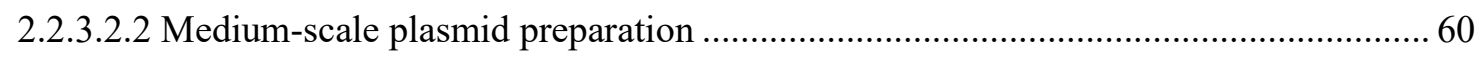

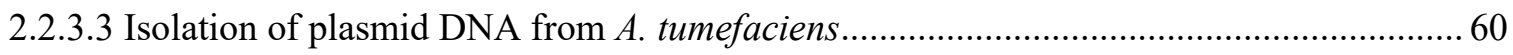

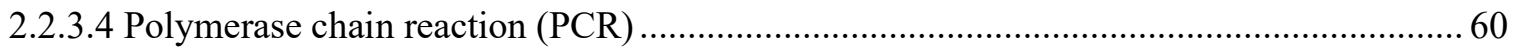

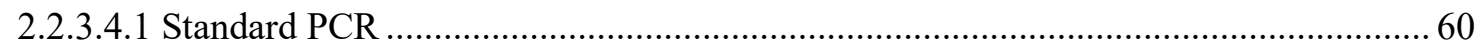

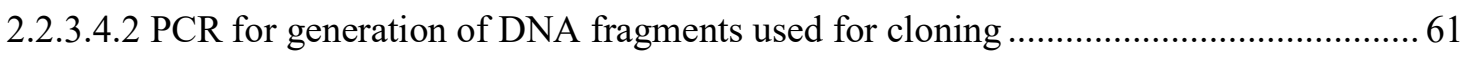

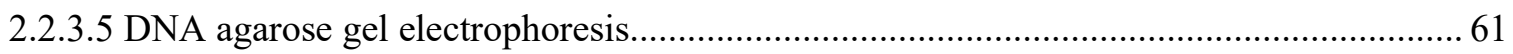

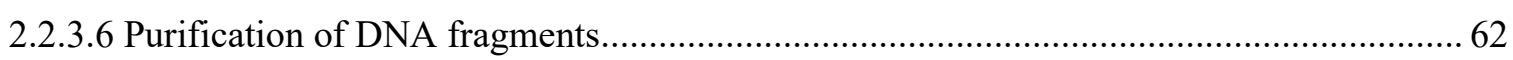

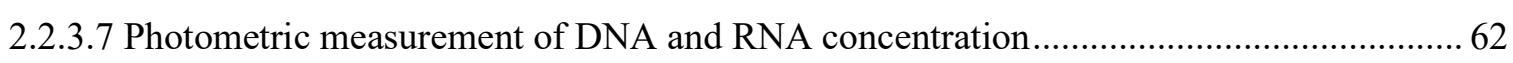

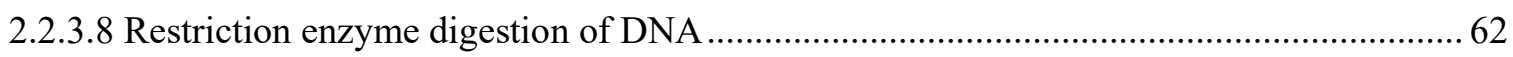

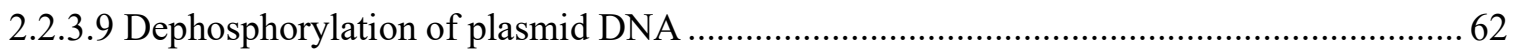

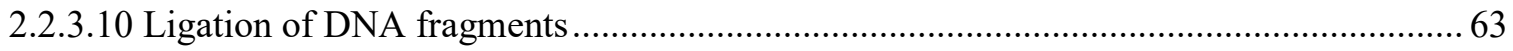

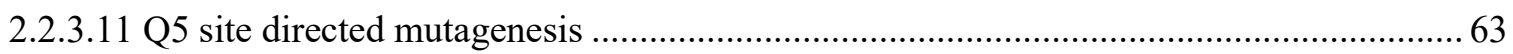

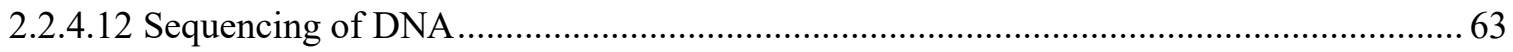

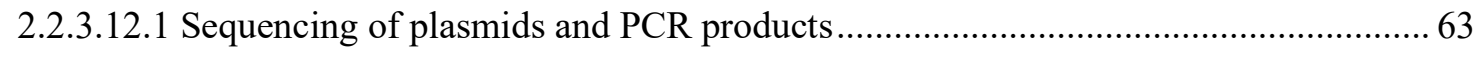

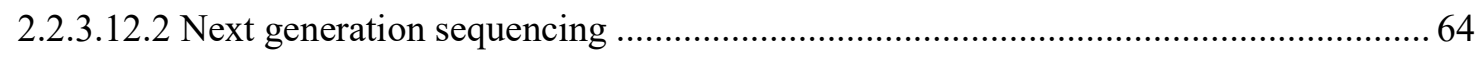

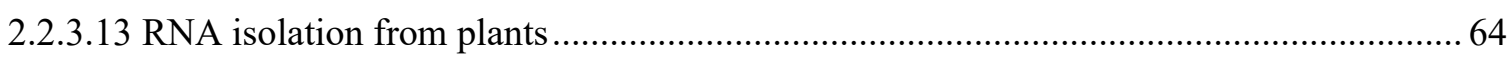

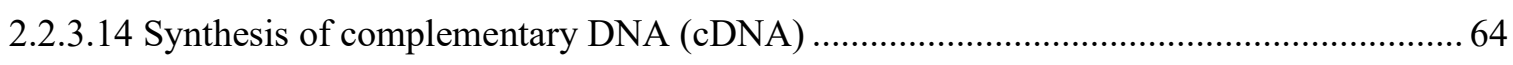

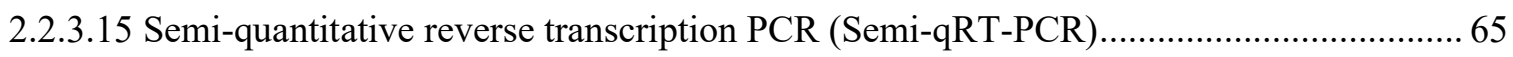

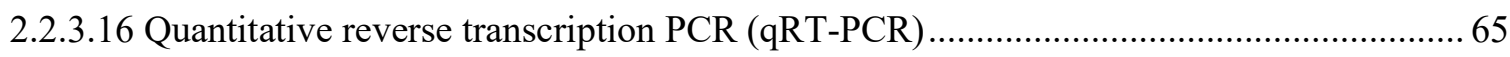

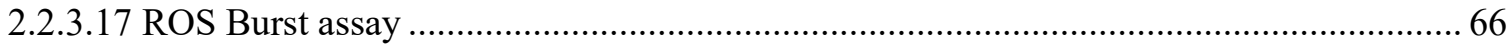

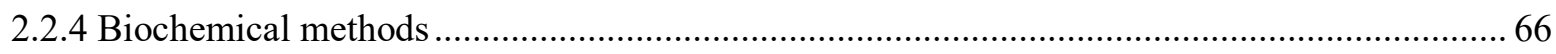

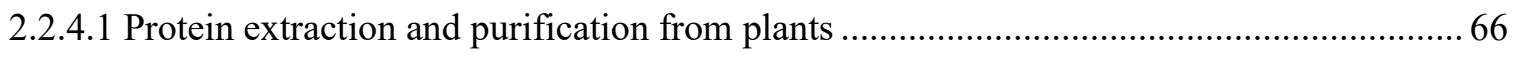

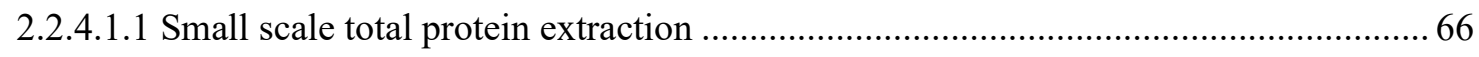

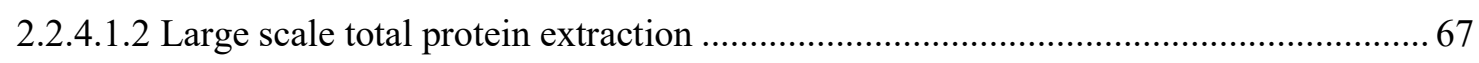

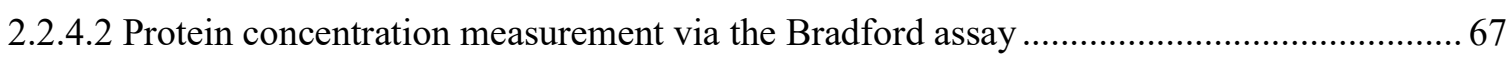

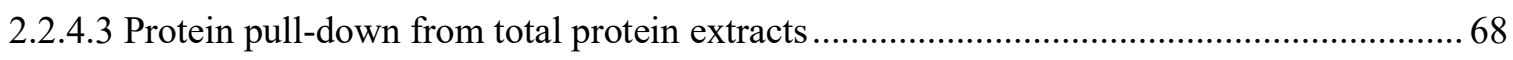

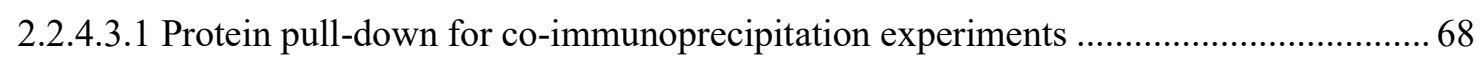

2.2.4.3.2 Protein pull-down for proteomics experiments and in vitro kinase assays.................. 68

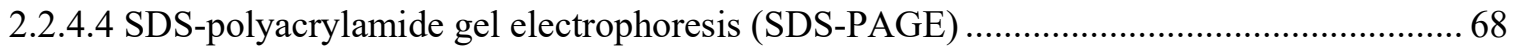

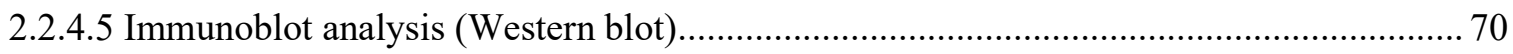




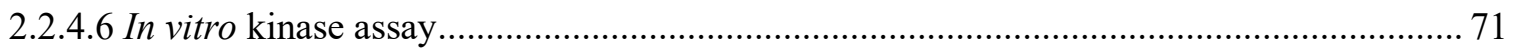

2.2.4.7 Coomassie staining of SDS-PAGE gels and PVDF membranes ...................................... 72

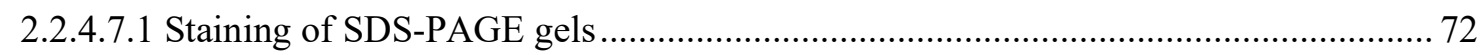

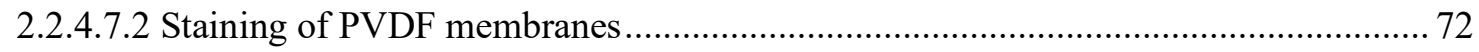

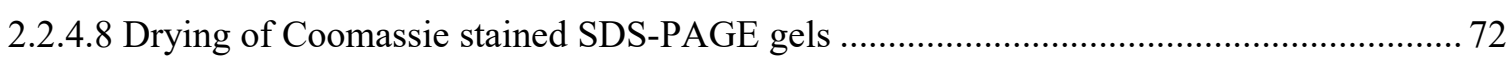

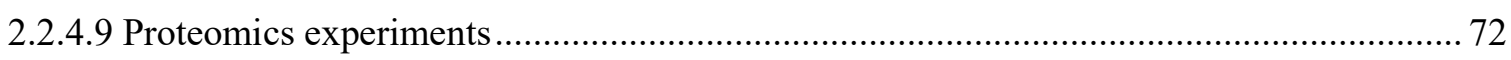

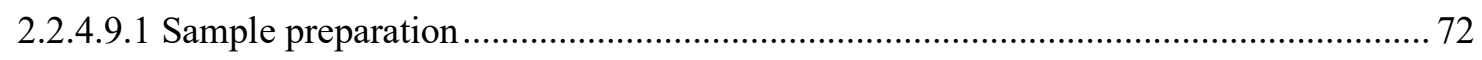

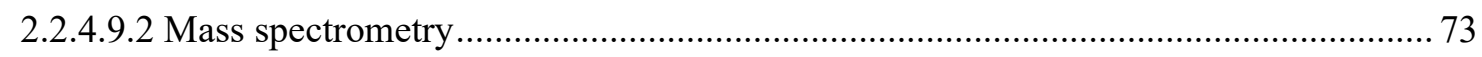

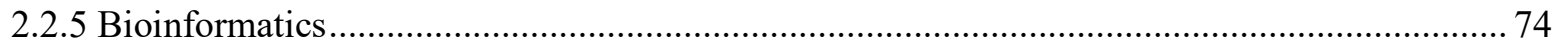

2.2.5.1 Mapping of Next Generation Sequencing (NGS) data to the Arabidopsis reference

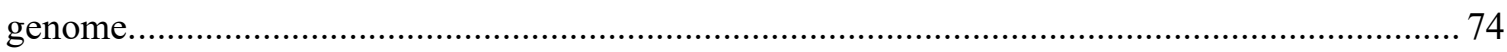

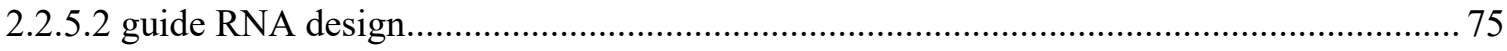

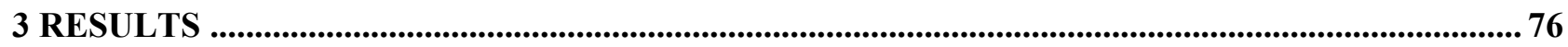

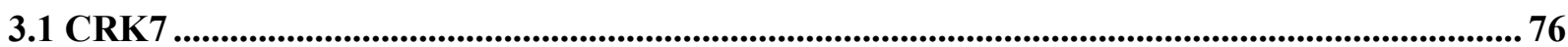

3.1.1 Mapping by sequencing revealed a single amino acid exchange in $C R K 7$ as possible

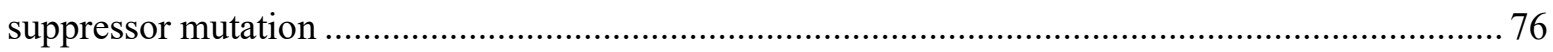

3.1.2 Confirmation of CRK 7 as a molecular component of cerk1-4 cell death induction................. 83

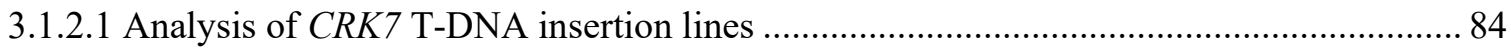

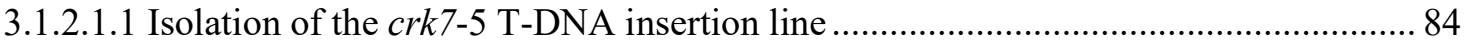

3.1.2.1.2 The crk7-5 T-DNA insertion suppresses the cerk1-4 cell death phenotype ............... 86

3.1.2.2 An independent $C R K 7$ mutant isolated in the cerk $1-4$ suppressor screen ......................... 87

3.1.2.3 Analysis of $C R K 7$ knockout lines generated with the CRISPR/ Cas9 system................... 90

3.1.2.3.1 Isolation of the cerk1-4 crk7-7 and cerk1-4 crk7-8 full deletion lines ....................... 90

3.1.2.3.2 The crk7-7 deletion suppresses the cerkl-4 phenotype ............................................. 92

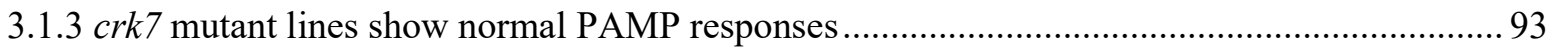

3.1.3.1 crk7 mutants show WT-like PAMP induced ROS accumulation ..................................... 93

3.1.3.2 crk7 mutants show WT-like PAMP induced MAPK phosphorylation................................ 95

3.1.3.3 PAMP-induced WRKY30 and WRKY53 induction is normal in $c r k 7-5 \ldots \ldots \ldots \ldots \ldots \ldots \ldots \ldots \ldots . . . . . .95$

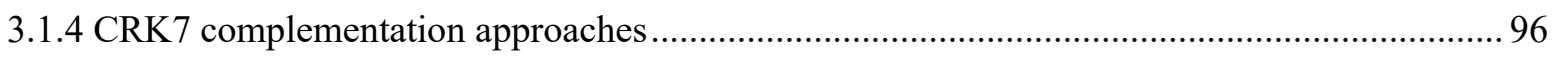

3.1.4.1. Complementation by expressing CRK7 in cerk1-4 crk7-4 and cerk1-4 crk7-5 ............... 97

3.1.4.1.1 Expression of untagged CRK7 in cerk1-4 crk7-4 and cerk1-4 crk7-5 .................... 97

3.1.4.1.2 Expression of Citrine- or FLAG-tagged CRK7 in cerk1-4 crk7-4 and cerk1-4 crk7-5

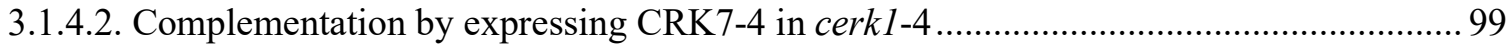

3.1.4.2.1 Expression of untagged CRK7-4 in cerk1-4.......................................................... 99

3.1.4.2.2 Expression of CRK7-4-Citrine in cerk1-4 …......................................................... 100 
3.1.5 Analysis of CRK7 and CRK7-4 subcellular localization 101

3.1.5.1 Analysis of CRK7-Citrine and CRK7-4-Citrine subcellular localization 101

3.1.5.1.1 CRK7-Citrine and CRK7-4-Citrine subcellular localization in transiently transformed Nicotiana benthamiana 101

3.1.5.1.2 CRK7-Citrine and CRK7-4-Citrine subcellular localization in stably transformed Arabidopsis. 103

3.1.5.2 Analysis of CRK7-mCitrine and CRK7-4-mCitrine subcellular localization. 105

3.1.5.2.1 CRK7-mCitrine and CRK7-4-mCitrine subcellular localization in transiently transformed Nicotiana benthamiana

3.1.5.2.2 Co-infiltration of CRK7-mCitrine and $c r k 7-4$-mCitrine with cellular markers in $N$. benthamiana

3.1.5.2.3 CRK7-mCitrine and CRK7-4-mCitrine subcellular localization in stably transformed Arabidopsis.. 109

3.1.6 Analysis of CRK7 and CRK7-4 protein function 110

3.1.6.1 A high molecular weight band is present in CRK7 Western Blots. 111

3.1.6.2 CRK7 interacts with CRK7 whereas CRK7-4 is unable to interact with CRK7-4 113

3.1.6.3 Proteomics experiments identified phosphorylation sites in CRK7 and CRK7-4 114

3.1.6.3.1 CRK7 is highly phosphorylated in the kinase domain 115

3.1.6.3.2 CRK7-4 interacts with ER chaperones involved in protein folding in N. benthamiana

3.1.6.4 CRK7 is an active kinase and phosphorylation of the activation and $\mathrm{P}+1$ loop is necessary for kinase activity .....

3.2 CRK43 123

3.2.1 Mapping by sequencing revealed a splicing mutation in CRK43 as possible cerk1-4 suppressor mutation . 123

3.2.2 Confirmation of CRK43 as a molecular component of cerk1-4 cell death induction 129

3.2.2.1 Analysis of crk43 T-DNA insertion lines 130

3.2.2.1.1 Isolation of the crk43-1 and crk43-3 T-DNA insertion lines 130

3.2.2.1.2 The crk43-3 T-DNA insertion suppresses the cerkl-4 cell death phenotype ............ 132

3.2.2.2 Expression of CRK43 in cerk1-4 crk43-2 induces cell death ........................................ 133

3.2.3 crk43 mutant lines show normal early PAMP responses ................................................... 135

3.2.3.1 CERK1 phosphorylation upon chitin perception is not impaired in crk43 mutants ........ 136

3.2.3.2 crk43 mutants show WT-like PAMP induced ROS accumulation................................... 136

3.2.3.3 crk43 mutants show WT-like PAMP induced MAPK activation ..................................... 137

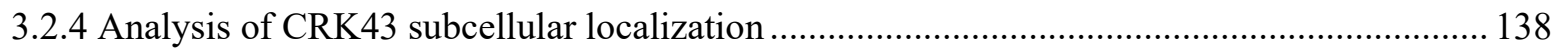

3.2.4.1 Analysis of CRK43-mCitrine localization after transient expression in N. benthamiana 139

3.2.4.2 Analysis of CRK43-mCitrine localization in Arabidopsis .............................................. 141 
3.3 Isolation of a novel pad4 mutation that is able to suppress cerk1-4 dependent cell death ..... 142

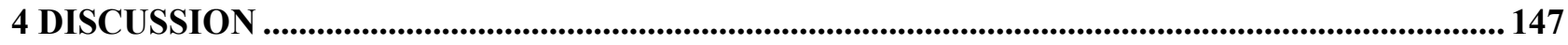

4.1 CRK7

4.1.1 crk7 mutants show semi-dominant phenotypes ............................................................. 149

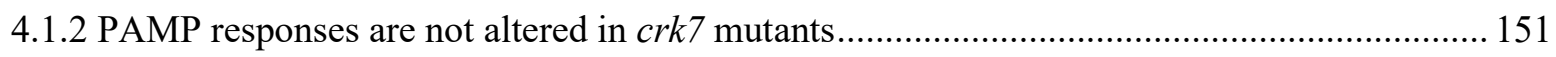

4.1.3 CRK7 localizes predominantly to the plasma membrane and CRK7-4 is restricted to the ER

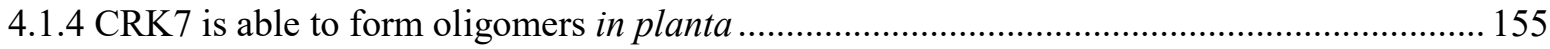

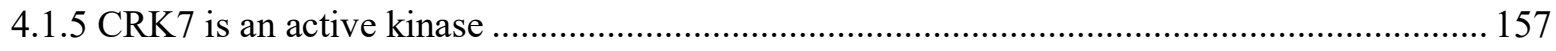

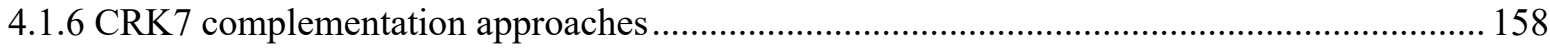

4.2 CRK43 ........................................................................................................................................................ 160

4.2.1 Confirmation of crk43-2 as cerkl-4 suppressor mutation.................................................... 160

4.2.2 PAMP responses are not altered in crk43 mutants................................................................ 161

4.2.3 CRK43 localizes to the cell periphery, nucleus and nucleolus ........................................... 162

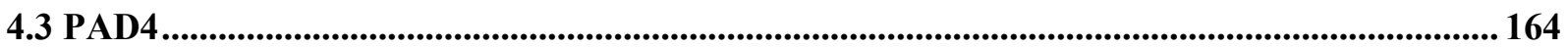

4.4 Potential roles of CRK7 and CRK43 in cerk1-4 cell death signaling.........................................165

4.5 Outlook ............................................................................................................................................................. 168

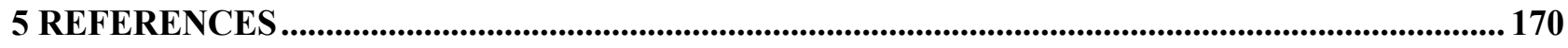

6 SUPPLEMENTAL MATERIAL .................................................................................................................. 188

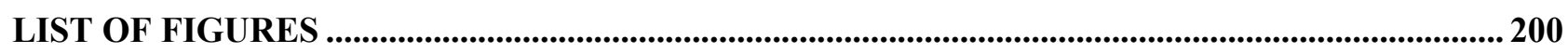

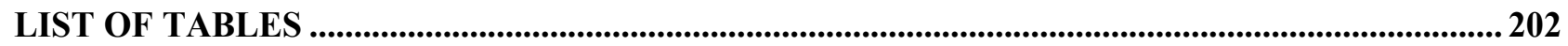

LIST OF SUPPLEMENTAL FIGURES AND TABLES .......................................................................203

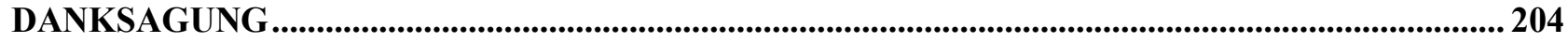




\section{Introduction}

Plants are constantly exposed to different biotic and abiotic stress factors. As sessile organisms plants cannot evade those stresses. Therefore it is essential that plants detect potential invaders such as bacteria, fungi or oomycetes and rapidly activate cellular defense responses (Jones and Dangl, 2006; de Wit, 2007). Since plants lack an adaptive immune system, they rely on their innate immunity that includes a variety of defensive barriers and inducible responses (Dodds and Rathjen, 2010). The plant immune system has been shaped by a co-evolutionary arms race of plants and their pathogens, resulting in plants resistant against most pathogens and susceptible to a small number of adapted microbes (Jones and Dangl, 2006; Postel and Kemmerling, 2009).

\section{$\underline{1.1 \text { Plant innate immunity }}$}

Microbial pathogens invading a plant need to overcome physical barriers such as the cuticle and the plant cell wall at first (Houston et al., 2016). Once a pathogen manages to overcome these barriers, it faces the plant innate immune system, which was suggested to be a two-layer system (Jones and Dangl, 2006). In the first layer of defense, plants recognize potential invaders via conserved so called pathogen or microbe associated molecular patterns (PAMPs/ MAMPs). These patterns are highly conserved molecular structures that are present in a whole class of microbes but absent from the host and cannot easily be modified as they are typically essential components of the pathogen (de Wit, 2007; Dodds and Rathjen, 2010). Additionally, plants can perceive host-derived molecules that are only present upon pathogen attack or cell damage, known as damage/ danger-associated molecular patterns (DAMPs) (Boller and Felix, 2009). MAMP or DAMP perception takes place via specialized pattern recognition receptors (PRRs) localized in the plasma membrane. Upon recognition of these conserved molecular patterns, PRRs trigger a number of signaling events and defense responses leading to MAMP/ DAMP-triggered immunity (PTI) (Jones and Dangl, 2006; de Wit, 2007; Dangl et al., 2013) (Figure 1, 1).

Both, MAMP and DAMP perception, leads to a range of largely overlapping defense responses including early responses like the generation of reactive oxygen species (ROS) via NADPH oxidases localized in the plasma membrane (PM), alterations in the ion fluxes at the PM including induction of $\mathrm{Ca}^{2+}$ signaling and protein phosphorylation including the induction of intracellular mitogen-activated protein kinase (MAPK)-signaling cascades (Boller and Felix, 2009; Yu et al., 2017). Later responses induced by MAMP/ DAMP perception incorporate transcriptional reprogramming of defense related genes and callose deposition at the cell wall (Boller and Felix, 2009; Yu et al., 
2017). Early and late responses together lead to PAMP-triggered immunity (PTI) (Jones and Dangl, 2006).

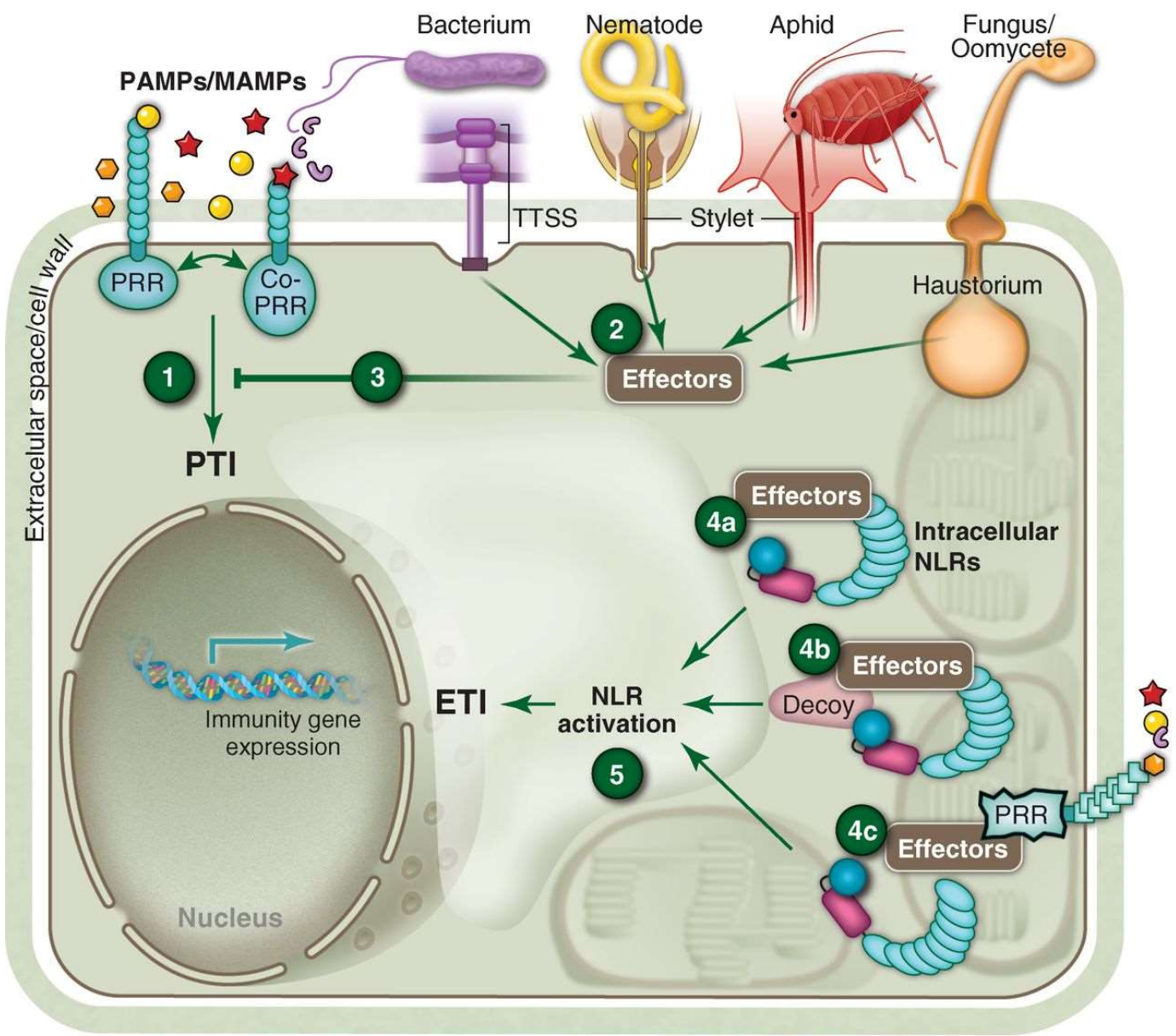

Figure 1: Schematic overview of the two-layered plant immune system.

Pathogen/ microbe associated molecular patterns (PAMPs/ MAMPs) are perceived by plant pattern recognition receptors (PRRs) that typically interact with co-receptors (Co-PRR) to induce pattern triggered immunity (PTI) (1). Pathogens secrete effector molecules into the plant cell (2) that suppress PTI responses (3). Plant nucleotide-binding leucine-rich repeat (NLR) type resistance (R) proteins recognize effector molecules either directly (4a) or indirectly. Indirect recognition may take place by guarding decoy proteins that mimic host effector targets (4b) or by sensing alterations made to host effector targets (4c). Recognition of effectors by NLRs (5) leads to effector-triggered immunity (ETI). Figure adapted from Dangl et al., 2013.

To overcome this first layer of plant innate immunity and for effective plant colonization, pathogens evolved small so called effector molecules (Figure 1,2). Many pathogen effectors are able to suppress PTI at various levels leading to effector-triggered susceptibility (ETS) in the host (Jones and Dangl, 2006; Thordal-Christensen, 2020) (Figure 1, 3). To induce ETS, pathogens have evolved specific mechanisms either for secretion of effector molecules into the apoplastic space or direct transfer of effectors into the cytoplasm of the host cell (de Wit et al., 2009).

Gram-negative bacteria such as Pseudomonas strains use their type III secretion system (TTSS) to deliver effector molecules into the host cell (Figure 1). Pseudomonas strains lacking a functional 
TTSS are unable to deliver effector molecules and therefore cannot counteract the activated PTI (Jin et al., 2003).

Pathogenic fungi and oomycetes form a specialized penetration apparatus, the appressorium, to overcome the physical barrier of the plant cell wall, often with the help of carbohydrate lysing enzymes. To take up nutrients and to deliver effector molecules, the fungus forms a specialized infection structure, the haustorium (O'Connell and Panstruga, 2006; de Wit et al., 2009). Haustoriaderived effectors need to overcome several physical barriers of both pathogen and host before they reach the host cytoplasm, but the exact mechanisms how effector molecules are transferred from the fungus/oomycete into the host cytoplasm remains unclear (Panstruga and Dodds, 2009; Petre and Kamoun, 2014).

Once effectors have successfully been transferred into the host they are able to suppress PTI at various levels (Büttner et al., 2016; Selin et al., 2016). Effectors were shown to directly target PRRs and associated proteins, resulting in inhibition of PRR kinase activities or PRR degradation (Gimenez-Ibanez et al., 2009a; Macho et al., 2014; Zhou et al., 2014). Additionally, effectors interfere with basal defense responses by the modulation of MAPK cascades (Zhang et al., 2007; Cui et al., 2010) and ROS accumulation (Hemetsberger et al., 2012; Lozano-Durán et al., 2014). Moreover, some effectors bind to or act as E3 ligases for degradation of specific plants proteins (Park et al., 2012; Ishikawa et al., 2014), modulate phytohormone signaling processes (Chen et al., 2007; Zhou et al., 2015) or plant gene expression (Le Roux et al., 2015; Sarris et al., 2015), block plant proteases (Müller et al., 2013) or lignin biosynthesis (Tanaka et al., 2014) to overcome PTI.

In a co-evolutionary arms-race to counteract ETS, plants developed so called resistance (R) proteins. $\mathrm{R}$ proteins recognize either the presence of effector molecules or modifications caused by effectors, leading to effector triggered immunity (ETI) (Jones and Dangl, 2006; Dangl et al., 2013) (Figure 1, 5). Nucleotide-binding leucine-rich repeat (NLR) receptors are typical plant R proteins. NLR receptors are structurally related to nucleotide-binding oligomerization domain (NOD)-like immune receptors in mammals (Ausubel, 2005; Cui et al., 2015). Plant NLR receptors are divided into two major subclasses according to their N-terminal domains. NLRs with an N-terminal Tollinterleukin 1 receptor (TIR) domain are called TIR-NLRs or short TNLs, whereas NLRs with a coiled-coil (CC) domain are known as CC-NLRs, in short CNLs (Cui et al., 2015). NLR-mediated effector detection can be either direct or indirect. Whereas direct recognition based on physical binding of effectors by NLRs (Dodds et al., 2006; Cui et al., 2015) (Figure 1, 4a) is relatively rare, indirect recognition has been observed more frequently. For indirect recognition of effectors, the $\mathrm{R}$ protein can associate with an effector target involved in plant innate immunity and sense effectormediated alterations on this protein (Figure 1, 4c). Another way of indirect effector recognition is 
guarding of a so called decoy protein. Decoy proteins mimic effector targets but are not known to fulfill any other cellular functions. R proteins sense effector-mediated alterations of this decoy (Figure 1, 4b) (van der Hoorn and Kamoun, 2008; Dangl et al., 2013; Cui et al., 2015; Noman et al., 2019). Recognition of effector molecules by $\mathrm{R}$ proteins leads to effector triggered immunity of the host plant. Therefore these effector molecules are termed avirulence (Avr) factors (Jones and Dangl, 2006).

For downstream ETI signaling many CNLs recruit NON-RACE-SPECIFIC DISEASE RESISTANCE 1 (NDR1) whereas TNLs rely on ENHANCED DISEASE SUSCEPTIBILITY 1 (EDS1) (Wiermer et al., 2005). ETI includes defense processes already known from PTI such as accumulation of ROS, activation of MAPK signaling cascades and defense gene induction. Moreover, ETI is often associated with a hypersensitive response (HR), a special type of local cell death to restrict the growth of biotrophic pathogens (Jones and Dangl, 2006; Cui et al., 2015)

Recently the activation mechanism for the NLR HOPZ-ACTIVATED RESISTANCE 1 (ZAR1), which induces ETI in response to several bacterial effectors, was defined (Wang et al., 2019a, b). In the resting state, ZAR1 interacts with one of at least three receptor-like cytoplasmic kinases (RLCKs) with a pseudokinase domain. Decoy proteins modified by different pathogen effectors can bind to the ZAR1-RLCK dimer, resulting in a large conformational change that evicts ADP from the complex. Upon ATP binding the activated complex forms a pentamer, the so called resistosome that is structurally similar to mammalian inflammosomes. The resistosome is suggested to create a pore in the plasma membrane leading to cell death (Wang et al., 2019a, b; Dangl and Jones, 2019).

By ETR and HR a long-lasting broad spectrum resistance can be induced in uninfected tissue, called systemic acquired resistance (SAR). SAR is associated with the accumulation of salicylic acid (SA) and the expression of PATHOGENESIS-RELATED (PR) genes (Malamy et al., 1990; Cao et al., 1998; Durrant and Dong, 2004; Fu and Dong, 2013). NON-EXPRESSOR OF PATHOGENESIS-RELATED GENES 1 (NPR1) is a key regulator of SA-signaling in Arabidopsis (Backer et al., 2019). In SA naïve cells, NPR1 predominantly forms oligomers in the cytoplasm. Besides these, also some monomeric NPR1 proteins are present and translocated into the nucleus to prevent SAR-gene expression. Elevated cellular SA concentration lead to the disassembly of the NPR1 oligomer. Monomeric NPR1 is then translocated into the nucleus and SA-binding leads to a conformational change that allows the interaction of NPR1 with transcription factors for induction of SAR genes (Backer et al., 2019).

The plant hormone SA is not only required for SAR induction, but contributes also to PTI and ETI. SA is synthesized by the ISOCHORISMATE SYNTHASE 1 (ICS1) and the induction of SA syn- 
thesis is controlled by complex transcriptional regulation of ICS1 expression (Seyfferth and Tsuda, 2014). In basal and TNL immunity EDS1 interacts with PHYTOALEXIN DEFICIENT 4 (PAD4) or SENESCENCE-ASSOCIATED GENE 101 (SAG101) to promote ICS1 expression and SA accumulation (Wagner et al., 2013; Cui et al., 2017).

\subsection{MAMP recognition via pattern recognition receptors}

Plants possess plasma membrane localized pattern recognition receptors of two different classes, the receptor-like kinases (RLKs) and the receptor-like proteins (RLPs) (Monaghan and Zipfel, 2012). RLKs consist of an ectodomain containing different motifs for ligand binding, a transmembrane domain and a cytoplasmic kinase domain for signal transduction. Among the $\sim 610 \mathrm{RLKs}$ encoded in Arabidopsis, approximately $25 \%$ are lacking the ectodomain and transmembrane domain and are therefore considered receptor-like cytoplasmic kinases (RLCKs) (Liang and Zhou, 2018). RLPs possess ectodomains similar to those of RLKs, a transmembrane domain or a GPIanchor for plasma membrane localization and a short cytoplasmic tail without signaling domains. Therefore it is likely that RLPs function in conjunction with RLKs for downstream signaling. Indeed it was shown that several leucine-rich repeat (LRR)-RLPs depend on association with SUPPRESSOR OF BIR1-1 (SOBIR1), an LRR-RLK (Gao et al., 2009; Zhang et al., 2013a; Liebrand et al., 2014; Domazakis et al., 2018). SOBIR1 possibly functions as a common adaptor kinase for several RLPs and the formed receptor complexes are suggested to operate as two-component RLKs (Gust and Felix, 2014; Domazakis et al., 2018).

RLPs and RLKs can not only function as cell surface-localized receptors, but also as co-receptors, helper receptors or scaffold proteins in receptor complexes to control different biological processes (Liang and Zhou, 2018). Both RLKs and RLPs were shown to form homo- as well as heterocomplexes for ligand recognition and the activation of downstream signaling (Macho and Zipfel, 2014). A recent model suggests that plant pattern recognition receptors, co-receptors and helper receptors are organized in a large and complex plant receptor network to translate pathogen recognition events into immune responses (Wu et al., 2018).

\subsubsection{LRR-RLK complexes and the recognition of peptide MAMPs}

A very well-studied LRR-RLK in plants is FLAGELLIN-SENSING 2 (FLS2), which perceives an N-terminal 22 amino acid epitope of bacterial flagellin, flg22 (Gómez-Gómez and Boller, 2000; Chinchilla et al., 2006). FLS2 binds flg22 via its 28 LRRs in the ectodomain. Bacteria lacking flagellin do not induce or induce only slight plant immune responses (Pfund et al., 2004; Sun et al., 
2006). Accordingly, FLS2-deficient plants show enhanced susceptibility towards adapted and nonadapted bacterial pathogens (Zipfel et al., 2004; Li et al., 2005; Hann and Rathjen, 2007). The FLS2 receptor is not only present in Arabidopsis but also in tomato, rice and tobacco, which makes the perception of flagellin an evolutionarily relatively old mechanism (Boller and Felix, 2009).

Another important LRR-RLK for peptide MAMPs is the elongation factor Tu receptor (EFR). EFR binds the bacterial elongation factor Tu and its conserved 18 amino acid N-terminal peptide elf18 with the 21 LRRs in the receptor ectodomain (Zipfel et al., 2006). EFR is restricted to Brassicaceae (Kunze et al., 2004) and efr mutant plants show enhanced susceptibility towards Agrobacterium tumefaciens as well as Pseudomonas syringae (Zipfel et al., 2006).

The most prominent DAMP-receptors in Arabidopsis are the plant elicitor protein (PEP) receptors PEPR1 and PEPR2 (Bartels and Boller, 2015). Both receptors perceive the 23 amino acid peptide PEP1, which is derived from its precursor PROPEP1 (Huffaker et al., 2006; Yamaguchi et al., 2010; Krol et al., 2010). PEP1 was shown to be involved in the induction of defense responses like $\mathrm{Ca}^{2+}$ signaling, MAPK phosphorylation and callose deposition. Therefore, PEP1 recognition leads to enhanced resistance against pathogen infection and defense against herbivore attack and wounding (Qi et al., 2010; Huffacker et al., 2013, Bartels et al., 2013).

The kinase active co-receptor BRI1-ASSOCIATED RECEPTOR KINASE 1 (BAK1)/ SOMATIC EMBRYOGENESIS RECEPTOR KINASE 3 (SERK3) is a critical component of many LRR-RLK complexes (Ma et al., 2016). BAK1 is a LRR-RLK with a short ectodomain and was originally identified as an interactor of the brassinosteroid receptor BRASSINOSTEROID INSENSITIVE 1 (BRI1) (Karlova et al., 2006). Independently from the function in BR signaling, BAK1 plays an important role in plant immunity. BAK1 was shown to rapidly form heteromeric complexes with FLS2 (Chinchilla et al., 2007; Heese et al, 2007), EFR (Roux et al., 2011) as well as PEPR1/ 2 (Postel et al., 2010) upon ligand perception. Heterodimerization upon ligand binding leads to transphosphorylation and signal transduction (Schulze et al., 2010) including transphosphorylation of the receptor-associated RLCK BOTRYTIS-INDUCED KINASE 1 (BIK1) that is subsequently released from the receptor complex and phosphorylates RBOHD leading to ROS burst and stomatal immunity (Lu et al., 2010; Zhang et al., 2010; Liu et al., 2013; Kadota et al., 2014). Nevertheless, bak1 mutants show only reduced responses to BR, MAMPs and DAMPs as there are two functionally redundant receptors, SERK1 and SERK4/ BAK1-LIKE1 (BKK1), present (Karlova et al., 2006, Chinchilla et al., 2007; Roux et al., 2011). 


\subsubsection{LysM-RLK complexes and the recognition of carbohydrate MAMPs}

The lysin-motif (LysM) is a carbohydrate binding domain initially identified in bacterial lysins. LysM proteins were shown to be involved in the degradation of bacterial cell walls composed of peptidoglycan (PGN) as well as fungal cell walls composed of chitin (Bateman and Bycroft, 2000; Buist et al., 2008). The presence of this motif in eukaryotes is varying and most likely the result of a horizontal gene transfer from bacteria (Bateman and Bycroft, 2000). Eukaryotic LysM domains were identified in fungal effectors as well as plant RLKs and RLPs involved in either defense or symbiosis pathways (Bolton et al., 2008; Buist et al., 2008; de Jonge and Thomma 2009). Plant LysM proteins were shown to bind poly- or oligosaccharides containing $\mathrm{N}$-acetylglucosamine (GlcNAc), such as chitin oligosaccharides, Nod-factors, myc factors and bacterial PGN (Buist et al., 2008; Antolín-Llovera et al., 2012; Gust et al., 2012, Antolin-Llovera et al., 2014).

\subsubsection{Chitin perception}

Chitin, the major component of fungal cell walls, is composed of $\beta$-1,4-linked $\mathrm{N}$-acetylglucosamine and serves as a highly conserved MAMP (Muzzarelli, 1978). Hence, poly- and oligomeric chitin as well as chitosan, a partially deacetylated form of chitin, are recognized by PRRs of several plant species (Boller and Felix, 2009).

In rice (Oryza sativa), CHITIN ELICITOR BINDING PROTEIN (CEBiP) was identified as chitin receptor (Kaku et al., 2006). CEBiP is a GPI-anchored RLP with three LysMs in its ectodomain (Hayafune et al., 2014, Gong et al. 2017). It was shown that two CEBiP molecules bind one chitin octamer from opposite sides and dimerize in a "sandwich type" manner. Each CEBiP molecule binds four GlcNAc monomers, sharing three of them with the other CEBiP of the homodimer. Therefore at least five GlcNAc moieties are necessary to form a stable dimer (Hayafune et al., 2014). As an RLP, CEBiP is lacking an intracellular kinase domain for signal transduction. Hence it forms a complex with CHITIN ELICITOR RECEPTOR KINASE 1 (OsCERK1), a functional LysM RLK without chitin binding ability (Shimizu et al., 2010; Shinya et al., 2012). Dimerization of OsCEBiP upon chitin binding recruits two OsCERK1 molecules into the complex and signal transduction is induced (Hayafune et al., 2014; Shinya et al., 2015). Silencing of either OsCEBiP or OsCERK1 leads to disruption of chitin induced immune responses (Kaku et al., 2006; Ao et al., 2014; Kouzai et al., 2014).

Two additional GPI-anchored LysM proteins with chitin-binding ability, LysM-CONTAINING PROTEIN 4 (OsLYP4) and OsLYP6, were shown to form complexes with OsCERK1 after chitin perception but associate with each other or with OsCEBiP in the absence of chitin (Ao et al., 2014). 
Plants silenced for OsLYP4 and OsLYP6 are affected in chitin and peptidoglycan induced defense signaling (Liu et al., 2012).

The Arabidopsis homologue to OsCEBiP, LYSM-CONTAINING RECEPTOR-LIKE PROTEIN 2 (LYM2), shows chitin-binding ability, but mutant plants are not impaired in chitin-induced defense responses (Shinya et al., 2012; Faulkner et al., 2013). Two closely to LYM2 related proteins, LYM1 and LYM3, have no chitin binding ability (Willmann et al., 2011) and a lym 1/2/3 triple mutant was also not affected in chitin-induced defense responses (Shinya et al., 2012). However it was shown that LYM2 mediates molecular fluxes through plasmodesmata by callose deposition in a chitin-dependent manner which is an important mechanism in defense against necrotrophic fungal pathogens (Faulkner et al., 2013; Cheval et al., 2020).

In Arabidopsis the OsCERK1 homologue LysM-RLK CERK1 was identified as a chitin receptor (Miya et al., 2007). Plants lacking a functional CERK1 receptor are completely insensitive to chitin and therefore more susceptible to fungal pathogens (Miya et al., 2007; Wan et al., 2008). Additionally an enhanced susceptibility of cerkl mutants towards Pseudomonas syringae infection was shown (Gimenez-Ibanez et al., 2009a). CERK1 contains three LysMs in its ectodomain (Miya et al., 2007). LysM2 was shown to directly bind oligomeric chitin, but all three LysM domains are necessary for chitin binding and form a tightly packed globular structure (Petutschnig et al., 2010; Liu et al., 2012). The extracellular domains of two CERK1 molecules homodimerize rapidly after chitin perception, leading to transphosphorylation of the kinase domains, which is visible as a bandshift in immunoblots (Petutschnig et al., 2010; Liu et al., 2012). Therefore, Arabidopsis CERK1 is directly involved in chitin sensing as well as in signal transduction, which is in contrast to OsCERK1 in rice. Petutschnig and colleagues (2010) reported that not only polymeric chitin but also chitin derivates with different lengths decreased CERK1 ability to bind to chitin beads, indicating that CERK1 can bind these chitin fragments. Whereas GlcNAc mono- or dimers were not able to induce a CERK1 mobility shift, tri- and tetramers as well as chitosan induced a weak band shift and chitin polymers with a polymerization degree greater than five induced a mobility shift at the same level to that observed upon treatment with polymeric chitin (Petutschnig et al., 2010). Contrary to these findings Liu and colleagues (2012) reported that chitin tetramers and pentamers can be bound by CERK1, but do not lead to CERK1 dimerization or phosphorylation.

Recently a "slipped sandwich" model for CERK1 chitin binding was proposed by Gubaeva and colleagues (2018). According to this model, the LysM2 domains of two CERK1 proteins form a binding pocket for one chitin heptamer. Each receptor is suggested to bind four GlcNAc moieties of the heptamer sharing the central GlcNAc unit with the other CERK1 molecule (Gubaeva et al., 
2018). In this model at least a GlcNAc pentamer is necessary for CERK1 dimerization since shorter chitin fragments would bind only to one CERK1 molecule, acting as an inhibitor for dimerization. Upon CERK1 phosphorylation immune responses including the accumulation of ROS, the activation of MAPK cascades and the upregulation of defense related genes including transcription factors are induced (Miya et al., 2007; Petutschnig et al., 2010). A CERK1 kinase dead version was unable to induce ROS burst and MAPK activation demonstrating CERK1 kinase activity to be required for PTI induction upon chitin treatment (Petutschnig et al., 2010).

Immunoblotting using an antibody (Gimenez-Ibanez et al., 2009a; Petutschnig et al., 2010) detecting an epitope at the $\mathrm{N}$-terminus of CERK1 revealed that not only the full length CERK1 protein (ca. $75 \mathrm{kDa}$ ) but also a shorter, soluble N-terminal fragment (33 kDa), is present. The shorter fragment has chitin-binding ability, accumulated both in senescent and in Blumeria graminis f.sp. hordeii $(B g h)$ infected WT plants and is most likely generated through ectodomain shedding near or within the transmembrane domain (Petutschnig et al., 2014). Ectodomain shedding is the limited proteolytic cleavage of transmembrane proteins to release the extracellular domain and known as an important regulatory mechanism in animals (Arribas and Borroto, 2002; Hayashida et al., 2010). Regarding plants there are currently a few studies pointing out that ectodomain shedding or related processes might be important regulatory mechanisms of receptor kinases (Lee et al., 2009 and 2013; Antolín-Llovera et al., 2014; Petutschnig et al., 2014; Pruitt et al., 2015; Zhou et al., 2019).

RLKs and RLPs typically form receptor complexes for signal transduction. Besides CERK1, also known as LysM CONTAINING RECEPTOR-LIKE KINASE 1, LYK1, Arabidopsis encodes four more LYK proteins. Two of them, LYK4 and LYK5, are able to bind chitin and were shown to be involved in chitin signaling (Petutschnig et al., 2010; Wan et al., 2012; Cao et al., 2014). Both contain three predicted LysM domains in the ectodomain, but in contrast to CERK1 are not enzymatically active kinases (Wan et al., 2012; Cao et al., 2014; Erwig et al., 2017). LYK4 was shown to play a minor role in chitin signaling since $l y k 4$ mutants were only slightly impaired in the induction of chitin-induced immune responses (Wan et al., 2012).

Interestingly, LYK5 was reported to bind chitin oligosaccharides with a much higher affinity than CERK1 (Cao et al., 2014). However, the necessity of LYK5 for chitin perception is unclear since contrary findings were published. One study (Cao et al., 2014) showed that CERK1 phosphorylation and MAPK activation were drastically reduced in lyk5 mutants upon chitin treatment. However, in other studies WT-like immune responses were observed in lyk5 mutants upon treatment with different chitin oligomers (Wan et al., 2012; Desaki et al., 2018; Gubaeva et al., 2018).

Moreover it was shown, that LYK5 homodimerizes in the absence of chitin, but the LYK5 homodimer dissociates upon chitin sensing and LYK5 heterodimerizes with CERK1. This was sug- 
gested to be crucial for CERK1 homodimerization and phosphorylation (Cao et al., 2014). Upon chitin treatment both LYK4 and LYK5 are directly phosphorylated by CERK1 leading to endocytosis of LYK5 and potentially also LYK4 whereas CERK1 itself remains at the plasma membrane (Erwig et al., 2017).

It is still under debate which receptor serves as the major chitin binding protein. Since LYK5 binds chitin oligosaccharides with a much higher affinity than CERK1, it was proposed that LYK5 acts as the major chitin binding protein and CERK1 functions as co-receptor necessary for signal transduction (Cao et al., 2014). Nevertheless, these binding affinity experiments were conducted with CERK1 and LYK5 proteins purified from E. coli (Cao et al., 2014) whereas direct CERK1 chitinbinding and receptor dimerization was demonstrated for CERK1 proteins expressed in insect cells (Liu et al., 2012). Since proteins expressed in E. coli are not glycosylated and lack disulfide bridges, the structure of these proteins might be altered and these experiments cannot be compared to each other.

Besides its role in chitin perception, CERK1 is also necessary for peptidoglycan (PGN) perception (1.2.2.3), but unable to bind PGN itself (Willmann et al., 2011) and was recently shown to be crucial for recognition of $\beta-1,3$ glucan, another fungal cell wall component (Mélida et al., 2018). Therefore it is suggested that CERK1 might act as a co-receptor inducing PTI responses upon treatment with several carbohydrate MAMPs (Mélida et al., 2018).

Nevertheless, cerk1-2 knockout plants were reproducibly shown to lack chitin-induced immune responses (Miya et al., 2007; Wan et al., 2008; Petutschnig et al., 2010; Wan et al., 2012; Cao et al., 2014; Desaki et al., 2018), whereas contradictory findings were published for lyk5 mutants as discussed before (Wan et al., 2012; Cao et al., 2014; Desaki et al., 2018; Gubaeva et al., 2018). The observed phenotypes in lyk5 mutants could be explained by functional redundancy of LYK4 and LYK5.

A recent model suggests that the chitin receptor is formed as a tetrameric complex composed of two CERK1 and two LYK5 proteins (Gubaeva et al., 2018). LYK5 dimerizes in the absence of chitin (Cao et al., 2014) and it was suggested that chitin binds first to the existing LYK5 dimer. Upon chitin binding to the LYK5 dimer, two CERK1 molecules are recruited for the formation of the tetrameric complex. In the absence of LYK5, CERK1 is suggested to form a homodimeric complex upon chitin perception that is sufficient to induce immune responses (Gubaeva et al., 2018). 


\subsubsection{Nod factor perception}

Nodulation (Nod)-factors are lipochitooligosaccharides secreted by nitrogen-fixing rhizobial bacteria (Lerouge et al., 1990; Antolín-Llovera et al., 2014; Via et al., 2016). Different rhizobial species exhibit Nod factors with varying chemical modification at several residues, determining host specifity (Oldroyd and Downie, 2008). Nod factors are important for nodule formation and therefore for establishment of symbiosis with legumes and are sensed by plant LysM RLKs (Radutoiu et al., 2003; Nakagawa et al., 2011; de Mita et al., 2014; Via et al., 2016).

The kinase active LysM RLK NOD FACTOR RECEPTOR 1 (NFR1) and kinase inactive NFR5 were identified to function in a complex for NF perception in Lotus japonicus (Madsen et al., 2011). NFR1 and NFR5 were shown to bind Nod factors with high affinity and $n f r l$ and $n f r 5$ mutant plants are unable to establish symbiosis with rhizobia (Radutoiu et al., 2003; Broghammer et al., 2012). SYMBIOSIS RECEPTOR-LIKE KINASE (SYMRK) interacts with both NFR1 and NFR5 and is proposed as co-receptor necessary for signal transduction (Antolín-Llovera et al., 2014). Recently a cytoplasmic kinase was identified as NFR5 interaction partner and named NFR5INTERACTING CYTOPLASMIC KINASE 4 (NiCK4) respectively (Wong et al., 2019). The current working model suggests that NiCK4 phosphorylates first NFR5 and then NFR1 in the presence of Nod factors. In turn, NFR1 phosphorylates NiCK4, leading to dissociation of NiCK4 from the complex and migration to the nucleus for signal transduction (Wong et al., 2019).

Two orthologues of NFR1 and NFR5, named NOD FACTOR PERCEPTION (MtNFP) and $M t \mathrm{LYK} 3$, are responsible for Nod factor perception in Medicago truncatula (Arrighi et al., 2006; Smit et al., 2007; Rey et al., 2013). Mtnfp mutants were impaired in NF perception, root hair deformation and initial NF responses (Amor et al., 2003), whereas Mtlyk3 mutants were impaired in the formation of infection threads and nodules (Limpens et al., 2003; Smit et al., 2007). Therefore $M t$ NFR1 is necessary to mediate early Nod factor sensing (Amor et al., 2003) whereas MtLYK3 functions in later stages of rhizobial infection (Smit et al., 2007). Interaction of the kinase active MtLYK3 (Smit et al., 2007) and the kinase inactive NFP (Arrighi et al., 2006) was observed only in a very narrow zone in infection nodules (Moling et al., 2014) and direct binding of Nod factors to $M t$ NFP and $M t$ LYK3 was not demonstrated yet (Fliegmann et al., 2016). However, another LysM-RLK, LysM RECEPTOR-LIKE KINASE 3 (MtLYR3), was identified as a high-affinity Nod factor binding protein (Fliegmann et al., 2013). $M t \mathrm{LYR} 3$ is an inactive kinase and $M t \mathrm{LYK} 3$ directly and efficiently interacts with and phosphorylates $M t$ LYR3 in the absence of Nod factors. In the presence of Nod factors this complex is disrupted (Fliegmann et al., 2016). Therefore, the current model suggests that $M t \mathrm{LYR} 3$ could act as a guard for $M t \mathrm{LYK} 3$ and prevent the formation of an 
active receptor complex with $M t$ NFP in the absence of Nod factors. Nod factor perfection leads to a modification of the $M t \mathrm{LYR} 3-M t \mathrm{LYK} 3$ interaction that potentially allows the functional interaction between $M t$ LYK3 and MtNFP (Fliegmann et al., 2016).

Arabidopsis is unable to establish symbiosis with rhizobia. Nevertheless, the Arabidopsis LysM RLK $A t$ LYK3 was suggested to detect NFs which leads to suppression of PTI (Liang et al., 2013).

\subsubsection{Peptidoglycan perception}

Peptidoglycan (PGN) is an essential cell wall component of gram negative and gram positive bacteria and is recognized as MAMP in different plant species (Gust, 2015). PGN is composed of alternating $\beta$-1,4-linked $\mathrm{N}$-acetylglucosamin and $\mathrm{N}$-acetylmuramic acid moieties cross-linked via peptidyl "bridges" and therefore structurally related to chitin (Lovering et al., 2012).

In rice, the LysM-CONTAINING PROTEINs OsLYP4 and OsLYP6 bind chitin as well as petidoglycan. Accordingly, lyp4 and lyp6 mutant plants show enhanced susceptibility towards fungal and bacterial pathogens (Liu et al., 2012a). Similarly, Oscerk1 mutant plants are not only impaired in chitin but also in peptidoglycan-induced defense signaling (Ao et al., 2014; Kouzai et al., 2014). As OsCERK1 has no peptidoglycan binding ability itself, the current model suggests that LYP4 and LYP6 form hetero-oligomers in the absence of MAMPs, disassociate upon PGN or chitin binding and associate with $O s C E R K 1$ to form an activation complex for signal transduction (Ao et al., 2014).

Arabidopsis cerkl mutant plants are not only blocked in chitin responses but show an enhanced susceptibility against Pseudomonas syringae infection (Gimenez-Ibanez et al., 2009a; Willmann et al., 2011). CERK1 is unable to bind peptidoglycan, but two LysM RLPs, LYM1 and LYM3, were shown to have peptidoglycan binding ability and lym 1 as well as lym3 mutant plants are impaired in peptidoglycan perception (Willmann et al., 2011). LYM1 and LYM3 both localize to the plasma membrane and a model has been proposed where LYM1 and LYM3 associate with CERK1 to mediate signal transduction upon PGN binding (Willmann et al., 2011). PGN perception triggers neither CERK1 dimerization nor phosphorylation, suggesting that in chitin and peptidoglycan perception different mechanisms are used for signal transduction (Petutschnig et al., 2010; Liu et al., 2012b). 


\subsection{A CERK1 mutant with enhanced pathogen-induced cell death and altered receptor pro-}

\section{cessing}

In order to identify new components involved in Arabidopsis non-host resistance (NHR) against non-adapted powdery mildews, a forward genetic screen was performed. After chemical mutagenesis of Col-3 gll seeds with ethyl methane sulfonate (EMS), plants with altered interaction phenotypes towards the non-adapted powdery mildew fungi Bgh were obtained (Petutschnig et al., 2014). In one line with such an altered phenotype upon $B g h$ infection, a leucine ${ }^{124}$ to phenylalanine amino acid exchange in the CERK1 ectodomain was identified as causative mutation and this mutant allele was named cerk1-4 (Petutschnig et al., 2014).

As a non-adapted powdery mildew, Bgh does not cause any macroscopically visible disease symptoms in infected Arabidopsis wildtype (WT) plants (Lipka et al., 2005). In contrast, cerk1-4 plants showed necrotic lesions and leaf chlorosis five to seven days upon Bgh infection. Clusters of dead cells were visible already three days post inoculation, which was never the case in WT plants or uninfected cerk1-4 plants. The cell death reaction is not, as in WT plants, restricted to attacked cells, but spreads to surrounding epidermis and subtending mesophyll cells in the mutant (Petutschnig et al., 2014). Upon infection with the compatible powdery mildews Golovinomyces orontii and Erysiphe cruciferarum similar cell death responses were observed. This exaggerated cell death leads to enhanced resistance against adapted powdery mildews as fungal mycelium was never detectable on cerk1-4 plants upon G. orontii and E. cruciferarum infection (Petutschnig et al., 2014 and E. Petutschnig, personal communication). Uninfected cerk1-4 plants are undistinguishable from WT plants until the age of five weeks, but later in development they show spontaneous cell death on older leaves and new leaves grow small and crinkly, resulting in a reduced rosette size.

The leucine ${ }^{124}$ to phenylalanine amino acid exchange is located in the second LysM of the CERK1 ectodomain (Petutschnig et al., 2014). LysM2 was shown to directly bind chitin (Liu et al., 2012) and the mutated leucine ${ }^{124}$ residue is conserved in the central LysM domain among all Arabidopsis LysM-RLKs and LysM-RLPs (Petutschnig et al., 2014). This high conservation suggests an important structural function. Mutation of this residue to a bulkier amino acid might interfere with the tight packing of the three LysM domains in CERK1 causing conformational changes. To address this, another mutant, cerk1-5, was generated. In cerk1-5 two amino acids in the first $\beta$-strand of LysM1 that are close to the cerk1-4 site, were exchanged for amino acids with bulkier side chains but similar properties. Expression of CERK1-5 in the cerk1-2 background resulted in cerk1-4-like 
cell death, suggesting that the enhanced cell death reaction is indeed caused by CERK1 conformational changes (Petutschnig et al., 2014).

Upon infection with powdery mildews, cerkl-4 plants accumulate the cell death promoting phytohormone salicylic acid (SA) to significantly higher levels than WT plants. Enhanced SA accumulation was also observed in senescent plants, suggesting the exaggerated cell death in cerk1-4 to be SA dependent. Indeed, cerk1-4 double mutants defective in genes necessary for SA synthesis or signaling, show a strongly reduced cerk1-4 phenotype (cerk1-4 sid2-1) or even WT-like (cerk1-4 pad4-1, cerk1-4 eds1-2) cell death responses upon Bgh infection (Petutschnig et al., 2014). Taken together, these results strongly suggest that the cerkl-4 phenotype depends on the accumulation of high SA levels.

Moreover it was shown that the nucleoporin NUP88, also known as MODIFIER OF SNC1 7 (MOS7) is necessary for cerkl-4 cell death since cerk1-4 mos 7-1 double mutants are lacking the exaggerated cell death response. This indicates that formation of the cerk1-4 cell death requires downstream signal transduction into the nucleus and MOS7-dependent nuclear accumulation of regulatory proteins (Genenncher et al., 2017).

As CERK1 is necessary for chitin perception in Arabidopsis (Miya et al., 2007), chitin-induced immune responses were investigated in cerk1-4 plants. CERK1-4-GFP shows normal localization at the plasma membrane and cerk1-4 plants are fully functional in chitin perception and chitininduced downstream signaling, suggesting that the cerkl-4 cell death phenotype is independent from the CERK1 chitin signaling function (Petutschnig et al., 2014). To investigate if the cerk1-4 phenotype requires CERK1 kinase activity, either a kinase-dead version of CERK1-4 or a CERK14 variant totally lacking the kinase domain were expressed in the cerkl-2 knockout background. These plants mimicked the cerk1-4 phenotype, suggesting that the cerk1-4 cell death is independent from CERK1 kinase activity and seems to be mediated by the N-terminal part of CERK1-4 (Petutschnig et al., 2014).

In Western Blots besides full length CERK1 a shorter N-terminal fragment is present that is most likely generated through ectodomain shedding. Interestingly, in CERK1-4 immunoblots only the full length receptor was present and the soluble $33 \mathrm{kDa}$ ectodomain fragment was never detected (Petutschnig et al., 2014). Nevertheless, it was shown, that a C-terminal fragment is present in cerk1-4 plants, suggesting that the ectodomain is cleaved off in cerk1-4 plants, but it does not accumulate (Petutschnig et al., 2014). As the structure of the ectodomain is affected in CERK1-4, the ectodomain fragment might be either unstable or targeted for degradation.

Currently the mechanism by which CERK1-4 elicits exaggerated cell death is unknown. One hypothesis is that the shed CERK1 ectodomain could act like an RLP sensing an unknown signal and 
mediating downstream signaling in interaction with an unknown RLK to prevent cell death induction. Since the cerk1-2 T-DNA insertion line does not show cerkl-4 like cell death, this is rather unlikely. An alternative explanation would be receptor guarding, like suggested for complexes containing BAK1 (Gao et al., 2009; Halter et al., 2014). For receptor guarding resistance proteins bind to a receptor or receptor complex to monitor its integrity. If the guarded protein or protein complex is absent or mutated, cell death is induced (Gao et al., 2009; Halter et al., 2014).

As the effective perception of chitin is important for plant health in an environment full of potential pathogens, it would be reasonable for the plant to monitor the integrity of CERK1. Plants may sense the altered characteristics or stability caused by the cerkl-4 mutation or sense altered interaction of CERK1-4 with complex partners and therefore trigger cell death. Moreover, specific degradation products of the shed ectodomain in cerkl-4 could be recognized by the plant as DAMPs, resulting in the activation of plant innate immunity and cell death.

\subsection{The cerk1-4 suppressor screen}

In order to unravel the process of cerk $1-4$ cell death induction, a forward genetic screen was started. For this, a population of cerk1-4 seeds was mutagenized using EMS to induce single nucleotide polymorphisms (SNPs) in the genome. The mutagenized M1 seeds were divided into 306 batches (containing approximately 60 seeds each) and propagated (M. Stolze and E. Petutschnig, unpublished). In the M2 generation, plants from individual batches were infected with the powdery mildew fungus Erysiphe cruciferarum. Plants that did not show a cerk1-4 cell death phenotype were isolated and named no-cerk1-4 cell death phenotype (noce). The suppressor phenotype was confirmed in the M3 generation and mutants were back-crossed to cerk1-4. In the F2 generation, segregating plants were phenotypically scored after E. cruciferarum infection and causative mutations were identified using a mapping by sequencing approach (Hartwig et al., 2012).

The causative suppressor mutations in two independent lines from this screen were both identified in the extra-large G-protein XLG2 (M. Stolze and E. Petutschnig, unpublished; C. Meusel, 2016). In one line a premature stop codon was inserted in XLG2, whereas in the other line a single amino acid exchange of a conserved glutamic acid to lysine (E293K) was identified as suppressor muta-

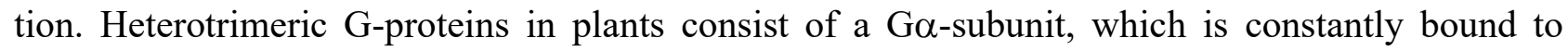
GTP, as well as a G $\beta$ - and G $\gamma$-subunit that form a dimer. According to the newest model, GTPbound G-proteins are activated by phosphorylation and fulfill downstream signaling functions until deactivated by dephosphorylation (Trusov and Botella, 2016). In Arabidopsis, extra-large Gproteins (XLGs) are encoded in addition to the canonical Ga-subunits and have roughly twice the size. Besides the C-terminal Go-like domain XLGs contain an N-terminal cysteine-rich region 
(Ding et al., 2008; Lee and Assmann, 1999). XLG2 has been shown to interact with canonical Gprotein $\beta \gamma$-dimers (Chakravorty et al., 2015; Maruta et al., 2015) and $\beta$ - and $\gamma$-subunits are required for full development of the cerk1-4 phenotype (E. Petutschnig, unpublished). Moreover, XLG2 was reported to localize to the cell periphery and the nucleus (Chakravorty et al., 2015; Maruta et al., 2015). Since the XLG2 E293K mutant isolated in the suppressor screen predominantly localized to the nucleus, the role of XLG2 localization for cerk1-4 cell death induction was studied in detail (J. Anders, unpublished). It was shown that $x \lg 2$ mutants that lost the cell periphery localization also lost the cerk1-4 cell death phenotype whereas loss of the nuclear localization had no effect on cell death induction (J. Anders, unpublished). This suggests that the cell periphery localization of XLG2 is necessary for cerk1-4 cell death signaling.

In this work, additional cerk1-4 suppressor mutants from the screen were investigated and two

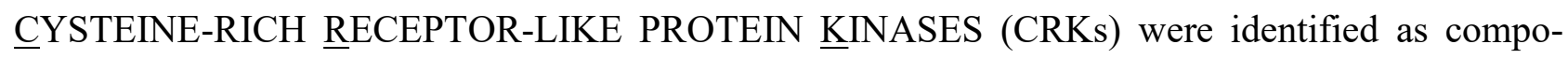
nents necessary for cell death induction. CRKs belong to the DUF26 domain containing proteins.

\subsection{DUF26 domain containing proteins in plants}

Plants encode large gene families of signaling proteins with extracellular domains including RLKs and secreted proteins, among them RLPs, that are necessary to respond to their environment (Shiu and Bleecker 2001 and 2003; Vaattovaara et al., 2019). Plant RLKs and RLPs are grouped into different families according to functional domains and sequence similarities (Shiu and Bleecker, 2001 and 2003; Fritz-Laylin et al., 2005). One of these domains is Domain of Unknown Function 26 (DUF26) which is also known as pFAM01657 (El-Gebali et al., 2019). The DUF26 domain is an extracellular domain containing a conserved motif with three cysteine residues separated by eight and two random amino acids $\left(\mathrm{C}-\mathrm{X}_{8}-\mathrm{C}-\mathrm{X}_{2}-\mathrm{C}\right)$ (Chen, 2001; Shiu and Bleecker, 2001). DUF26

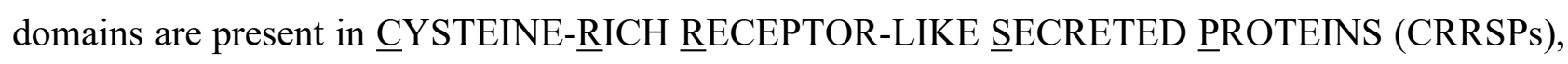

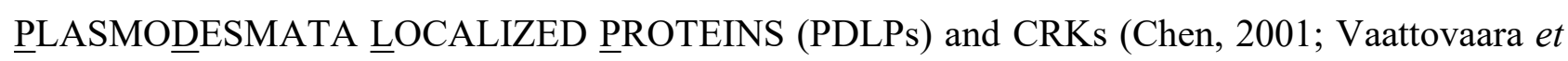
al., 2019). CRRSPs consist of a signal peptide followed by one or more DUF26 domains that are separated by a variable region (Figure 2A). PDLPs contain a signal peptide, two DUF26 domains and a transmembrane region with a short cytoplasmic extension and CRKs consist of a signal peptide, usually two DUF26 domains, a transmembrane region and an intracellular protein kinase domain (Figure 2A). Additionally, CRKs lacking the signal peptide, extracellular domain and transmembrane region were identified and are considered as $\underline{\text { CYSTEINE- } \underline{R} I C H ~ R E C E P T O R-L I K E}$ CYYTOPLASMIC KINASEs (CRCKs) (Vaattovaara et al., 2019; Figure 2A).

Analysis of 1409 DUF26 containing gene models from 32 plant species revealed that CRKs and CRRSPs are typically encoded in clusters and experienced both ancestral and recent lineage- 
specific tandem duplications (Vaattovaara et al., 2019). CRRSPs with a single DUF26 domain are suggested as the ancestral type of DUF26 proteins (Vaattovaara et al., 2019). CRKs likely evolved from a fusion of single DUF26 CRRSPs with the transmembrane region and kinase domain from LRR_clade_3 RLKs (Zulawski et al., 2014; Vaattovaara et al., 2019). The duplication to achieve the typical double DUF26 CRK configuration likely took place after this fusion. The two DUF26 domains diverged into distinct and evolutionary conserved forms in double DUF26 proteins. CRRSPs and PDLPs with two DUF26 domains most likely originated from CRKs through the loss of transmembrane regions and/ or kinase domains (Vaattovaara et al., 2019).

Structural information is available for the CRRSP GINKBILOBIN-2 (GNK2) from Ginkgo biloba (Miyakawa et al., 2009) and Arabidopsis PDLP5 and PDLP8 proteins (Vaattovaara et al., 2019). The single DUF26 domains in GNK2, PDLP5 and PDLP8 are structurally very similar. They consist of two $\alpha$-helices folding on top of a central antiparallel five-stranded $\beta$-sheet. Three of the $\beta$ strands are connected to one of the $\alpha$-helices via three cysteine bridges (Figure 2B-D) (Miyakawa et al., 2009; Vaattovaara et al., 2019). The cysteine-bridges were shown to be important for protein structure and stability as mutation of three of the conserved cysteine residues in PDLP5 to alanine resulted in structural instability of the protein and aggregate formation (Vaattovaara et al., 2019). The two DUF26 domains in PDLP5 and PDLP8 are connected by a structured loop and the $\beta$ sheets of DUF26-A and DUF26-B face each other, resulting in a claw-like shape (Figure 2D). The interface between the two DUF26 domains is not only conserved between PDLPs, but also among CRKs and CRRSPs with two DUF26 domains, suggesting that evolutionary distant proteins share a conserved three-dimensional structure (Vaattovaara et al., 2019). The claw-like shape of this three dimensional structure is similar to the overall protein structure of two fungal lectins (van Eerde $e t$ al., 2014; Zhang et al., 2017). Since these fungal lectins bind carbohydrate polymers, double DUF26 domains could be potentially also involved in binding of similar carbohydrates (van Eerde et al., 2014; Zhang et al., 2017; Vaattovaara et al., 2019). 
A
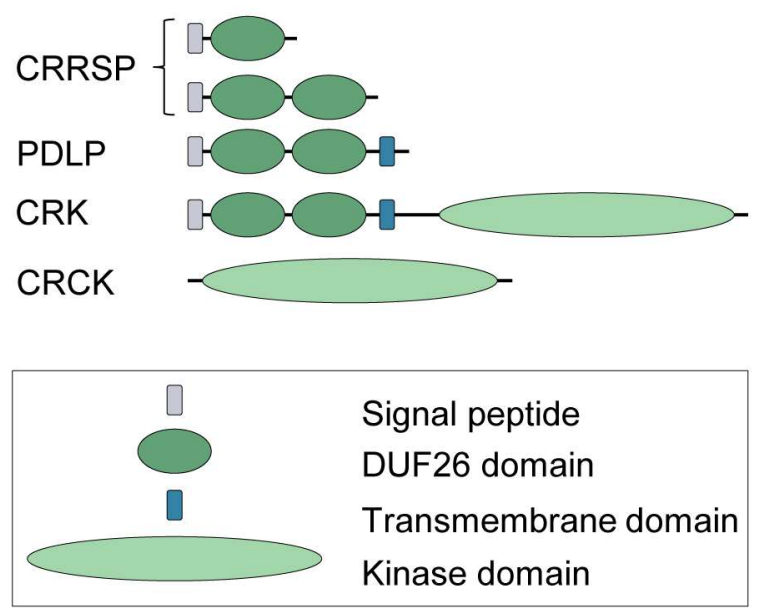

D

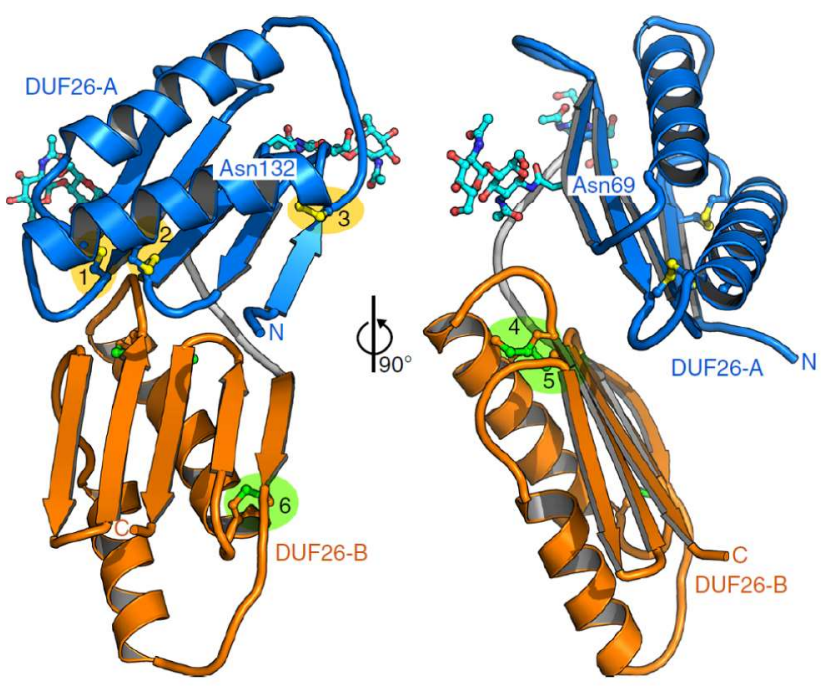

B

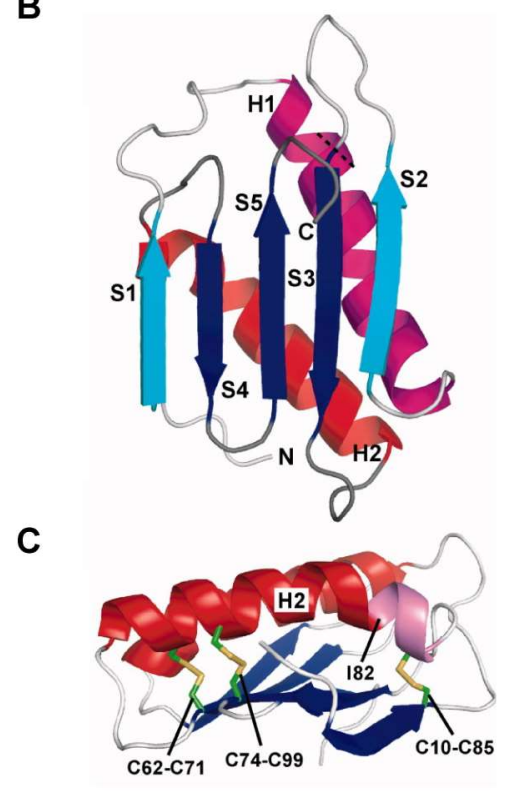

Figure 2: Domain compositions and protein structures of DUF26 domain containing proteins.

A: Overview of the domain organization in the most common DUF26 proteins. Adapted and modified from Vaattovaara et al., 2019. B and C: GNK2 crystal structure with two $\alpha$-helices (magenta and red) and a fivestranded antiparallel $\beta$-sheet (blue and cyan). Disulfide bridges between the $\mathrm{H} 2$ helix and the $\beta$-sheet are shown in $\mathrm{C}$ as stick models with the yellow stick indicating a covalent bond between two sulfurs. The disulfide bridge of Cys10-Cys85 causes a kink in the H2 helix (pink). Figures adapted from Miyakawa et al., 2009. D: Crystal structure of the PDLP5 ectodomain. The two DUF26 domains are shown as ribbon diagrams, colored in blue (DUF26-A) and orange (DUF26-B). Disulfide bridges are labeled 1 (Cys89-Cys98), 2 (Cys101-Cys126), 3 (Cys36-Cys113), 4 (Cys191-Cys200), 5 (Cys203-Cys228) and 6 (Cys148-Cys215). Figure adapted from Vaattovaara et al., 2019.

Alignment of all Arabidopsis CRKs with at least one DUF26 domain to GNK2 and PDLP5 (Figure $\mathrm{S} 1)$ revealed that the three cysteines belonging to the $\mathrm{C}-\mathrm{X}_{8}-\mathrm{C}-\mathrm{X}_{2}-\mathrm{C}$ motif are $100 \%$ conserved among all Arabidopsis CRKs in both DUF26 domains. Additionally three other cysteines that are necessary for the formation of disulfide bridges in GNK2 and PDLP5 (Miyakawa et al., 2009; Vaattovaara et al., 2019) are conserved in the first DUF26 domain of Arabidopsis CRKs (Figure 
S1A). Interestingly, in the second DUF26 domains of most Arabidopsis CRKs, one or two of the three cysteine residues that are necessary for the formation of disulfide bridges in PDLP5 (Vaattovaara et al., 2019) are missing (Figure S1B). This suggests that in these CRKs only two disulfide bridges connect the $\beta$-sheets with the $\alpha$-helix. The disulfide bridge connecting the first $\beta$-strand to the $\alpha$-helix is missing, suggesting that these CRKs might have a different domain structure compared to PDLP5.

Functions of the three classes of DUF26 domain containing proteins introduced here are reported in the next chapters with a focus on their roles in plant immunity.

\section{$\underline{\text { 1.5.1 Cysteine-rich receptor-like secreted proteins }}$}

The ancestral type of DUF26 containing proteins are CRRSPs with a single DUF26 domain (Vaattovaara et al., 2019). GNK2 from Ginkgo biloba is a single DUF26 domain CRRSP and it was shown that GNK2 is able to bind yeast mannan, but not other fungal cell wall components such as $\beta-1,3$ glucan and chitin (Miyakawa et al., 2014). Mannans are homopolymers of the hexose sugar mannose with different linkages. Yeast mannan has a $\alpha-1,6$ linked mannose backbone and $\alpha-1,2$ as well as $\alpha-1,3$ linked mannose side chains resulting in a highly branched structure containing up to 200 mannose residues (Lesage and Bussey, 2006). Mannans are not only present in fungal cell walls, but also in plant hemicelluloses. Moreover, N-glycosylated plant proteins typically contain mannose. Oligomannosidic and complex N-glycans are crucial for correct plant growth under stress conditions (Strasser, 2016).

In GNK2, the amino acid residues $\mathrm{Asn}^{11}, \mathrm{Arg}^{93}$, and Glu ${ }^{104}$, located in the upper part of the $\beta$-sheet, were shown to be crucial for mannose binding (Miyakawa et al., 2014). Moreover, GNK2 was shown to have antifungal activity as addition of GNK2 slowed down the yeast growth rate in liquid cultures. A mutated version of GNK2 in which $\mathrm{Arg}^{93}$ was exchanged to alanine was unable to bind mannan and showed only a minor slow-down of yeast growth, indicating that mannose-binding ability is required for GNK2 antifungal activity. GNK2 was shown to interact with $\alpha$-1,2-linked mannose moieties and did not affect the growth of a yeast strain unable to add $\alpha-1,2$-linked mannose moieties to N-linked carbohydrate chains (Miyakawa et al., 2014). Additionally, wildtype GNK2 was shown to have strong antifungal activity against the plant pathogens Fusarium oxysporum and Fusarium culmorum, whereas the GNK2 $\mathrm{Arg}^{93}$ to alanine mutant inhibited Fusarium growth only on a minor extent, confirming the findings for yeast anti-fungal activity (Sawano et al., 2007; Miyakawa et al., 2014). 
The two maize proteins AFP1 and AFP2 are both CRRSPs harboring two DUF26 domains and show high amino acid identity (Ma et al., 2018). The protein structure of single DUF26 domains in AFP1 was modelled and is not only structurally very similar to GNK2, also the amino acids for mannose binding are conserved. AFP1 WT was pulled down with mannose-agarose beads, whereas an AFP1 mutant version in which all conserved mannose binding moieties were exchanged to alanine, was unable to bind to mannose-agarose beads. AFP1 and AFP2 are both highly upregulated upon infection with the biotrophic pathogen Ustilago maydis and are supposed to be secreted into the apoplast (Ma et al., 2018). A secreted effector protein containing a large number of repetitive elements from $U$. maydis, repetitive secreted protein 3 (Rsp3), blocks the antifungal activity of AFP1 and AFP2. Accordingly, U. maydis strains lacking functional Rsp3 ( $\Delta$ Rsp3), show reduced virulence symptoms in Z. mays infections (Ma et al., 2018). AFP1 and the AFP1 mutant version lacking the conserved residues for mannose binding were incubated with $U$. maydis $\Delta \mathrm{Rsp} 3$ in axenic culture. The majority of $U$. maydis $\Delta \mathrm{Rsp} 3$ cells died after incubation with WT AFP1, but this rate was significantly reduced after incubation with AFP1 mutant proteins unable to bind mannose. Hence, AFP1 anti-fungal activity against $U$. maydis was demonstrated and mannose binding is required for this activity (Ma et al., 2018). Accordingly, maize plants silenced for AFP1 and partially silenced for AFP2 were significantly more susceptible to $U$. maydis $\Delta$ Rsp3 infection. As Rsp3 is localized to fungal hyphae, it most likely hinders AFP1 and AFP2 to bind to mannose in the fungal cell wall and therefore protects the fungus against the antifungal activity of AFP1 and AFP2 (Ma et al., 2018).

\section{$\underline{1.5 .2}$ Plasmodesmata localized proteins}

Plasmodesmata (PD) are membrane-lined cell wall channels that provide cytoplasmic continuity between plant cells. The system allows the exchange of small uncharged molecules between neighboring cells (Lee and Lu, 2011; Maule et al., 2011). PDs are involved in a number of processes, including the regulation of plant growth and development as well as cell differentiation (Zambryski and Crawford, 2000; Burch-Smith et al., 2011; Sager and Lee, 2018). Moreover, PDs are important for plant immunity, since a number of pathogens spread from cell to cell for plant colonization via PDs (Ueki and Citovsky, 2011; Tilsner et al., 2013; Kankanala et al., 2007).

PD-flux has to be tightly regulated to establish and maintain physiological gradients between cells and to prevent pathogen entry. One regulatory mechanism is the deposition of the plant polysaccharide callose to the neck of PDs resulting in PD closure (Lee and Lu, 2011; Maule et al., 2011; Cheval and Faulkner, 2018). Arabidopsis encodes eight PDLPs that are specifically recruited to plas- 
modesmata by a yet unknown mechanism. PDLPs were shown to function in developmental as well as immune processes (Thomas et al., 2008, Brunkhard and Zambrynski, 2017).

The expression of PDLP5 is upregulated upon P. syringae infection and SA treatment as well as in senescent plants. Constitutive overexpression of PDLP5 leads to growth inhibition, chlorosis and spontaneous lesions. Moreover, PDLP5 overexpressing plants accumulate significantly more SA than WT plants, suggesting the observed cell death to be SA dependent. Additionally this indicates the presence of a positive feedback loop (Lee et al., 2011). PDLP5 overexpression also leads to PD closure and enhanced callose deposition at the neck of plasmodesmata, whereas plasmodesmata were more open and less callose was deposited in a pdlp5 knockout mutant. These results show that PDLP5 regulates callose deposition and thus PD permeability. Moreover this integrates the control of plasmodesmata permeability into innate immunity and both processes were shown to be regulated by PDLP5 (Lee et al., 2011).

Knockout of $p d l p 1$ did not change PD-flux, but $p d l p 1 / 2$, and $p d l p 2 / 3$ double mutants show an increased molecular flux, suggesting functional redundancy between these PDLPs (Caillaud et al., 2014). PDLP1 is upregulated upon infection with the biotrophic pathogen Hyaloperanospora arabidopsidis (Hpa). In uninfected plants, PDLP1 localizes only to plasmodesmata, but upon Hpa infection PDLP1 surrounds unencased haustoria and remains associated with the haustorium as the encasement develops (Caillaud et al., 2014). PDLP1 overexpressing plants produced significantly more callose encasements and the $p d l p 1 / 2 / 3$ triple mutant, but not the single mutants, showed a reduced proportion of encased haustoria. However, the haustorium-associated localization is independent from the DUF26 domains as also a $p d l p 1$ mutant version lacking the DUF26 domains associated with haustoria (Caillaud et al., 2014).

Viruses often spread from cell to cell through plasmodesmata and encode movement proteins to expand the molecular flux through the PD channel for efficient plant colonization. The Grapevine fanleaf virus movement protein 2B was shown to directly interact with all eight PDLPs in Arabidopsis (Amari et al., 2010). This interaction enhances virus movement through plasmodesmata. Similarly, a movement protein of Cauliflower mosaic virus (CaMV) was shown to interact with PDLP1 and $p d l p 1 / 2 / 3$ mutant plants showed reduced symptoms upon CaMV infection (Amari et al., 2010).

Taken together, these results show that PDLPs play important roles in immunity against diverse pathogens.

DUF26 domain containing proteins were shown to bind mannose (Miyakawa et al., 2014; Ma et al., 2018), but the mannose binding moieties are not conserved in PDLP5 (Vaattovaara et al., 2019, Figure S1A). Interestingly, PDLP5 shows strong structural similarity to two fungal lectins that bind 
carbohydrate polymers (Vaattovaara et al., 2019). Therefore it was tested, if PDLP5 binds to mannose or other oligo- and polysaccharides, but PDLP5 was unable to bind to the investigated carbohydrates (Vaattovaara et al., 2019). Nevertheless, PDLP5 might be able to bind to other carbohydrates not investigated yet.

\section{$\underline{1.5 .3 \text { Cysteine-rich receptor-like protein kinases }}$}

Arabidopsis encodes more than 600 RLKs and among them CRKs form one of the largest subfamilies with 44 members (Chen, 2001; Wrzaczek et al., 2010). Most CRKs contain a signal peptide, two DUF26 domains in the ectodomain, a transmembrane domain and an intracellular kinase domain (Chen, 2001; Shiu and Bleecker, 2001; Wrzaczek et al., 2010). However, three CRKs (CRK43, CRK44 and CRK45) are lacking the complete extracellular domain and transmembrane

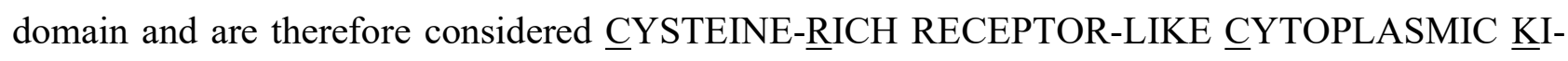
NASEs (CRCKs; Figure 3A) (Shiu and Bleecker, 2003; Bourdais et al., 2015). Besides this, the truncated CRK9 lacking the transmembrane and kinase domain (AT4G23170) as well as the putative pseudogene CRK35 (AT4G11500) are not considered as CRKs anymore (Wrzaczek et al., 2010; Bourdais et al., 2015).

Most CRKs are encoded in large gene clusters. The largest CRK cluster is located on the lower arm of chromosome four and contains 19 CRKs (CRK5-CRK24) (Chen, 2001; Idänheimo et al., 2014). The CRKs are divided into five different subgroups according to sequence similarities of the full length amino acid sequence (Figure 3A). Phylogenetic trees based on either the kinase domains or the ectodomains yielded very similar results. CRK45 could not be unambiguously assigned to any group (Bourdais et al., 2015)

To obtain evidence for possible functions of CRKs, their gene regulation was investigated in several studies. While some $C R K$ s are transcriptionally responsive to abiotic stimuli such as light stress, drought or high salinity (Wrzaczek et al., 2010; Marshall et al., 2012; Tanaka et al., 2012) the majority of studies report induced $C R K$ gene expression in response to pathogen infection or related treatments (Figure 3B). 


\begin{tabular}{|c|c|c|c|c|c|c|c|c|c|c|c|c|c|c|c|}
\hline \multirow{3}{*}{\multicolumn{2}{|c|}{ A }} & & \multicolumn{6}{|c|}{ Transcriptional regulation } & \multicolumn{5}{|c|}{ Knockout or knockdown phenotype } & \multicolumn{2}{|c|}{$\begin{array}{l}\text { D } \\
\text { Overexpression phenotype }\end{array}$} \\
\hline & & & \multirow{2}{*}{ SA } & \multirow{2}{*}{ Pst } & \multirow{2}{*}{$B g h^{8}$} & \multirow{2}{*}{ G.orontii ${ }^{8}$} & \multirow{2}{*}{ flg22 } & \multirow{2}{*}{$\mathrm{O}_{3}{ }^{9}$} & \multirow{2}{*}{$\begin{array}{c}\text { Susceptibility to } \\
\text { Pst }\end{array}$} & \multirow{2}{*}{$\begin{array}{c}\text { ROS production } \\
\text { upon flg22 } \\
\text { treatment }^{8}\end{array}$} & \multicolumn{2}{|c|}{\begin{tabular}{|l} 
Stomatal closure $^{8}$ \\
\end{tabular}} & \multirow{2}{*}{$\begin{array}{l}\text { Susceptibility to } \\
\text { G. orontio }\end{array}$} & \multirow{2}{*}{$\begin{array}{c}\text { Resistance to } \\
\text { Pst }\end{array}$} & \multirow{2}{*}{$\begin{array}{l}\text { Cell } \\
\text { death }\end{array}$} \\
\hline & & & & & & & & & & & fig22 treatment $\left.\right|_{\mathrm{Cl}}$ & chitin treatment & & & \\
\hline & 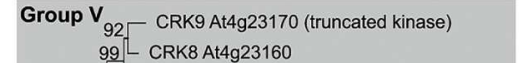 & $\begin{array}{l}\text { CRK9 } \\
\text { CRK8 }\end{array}$ & & & - & - & & - & -8 & - & & & & & \\
\hline & 92 CRK6 At4g23140 & CRK6 & $\bullet 1$ & $\bullet^{1}$ & - & - & & - & $-8,12$ & $\bullet$ & o & - & $\begin{array}{c}- \\
-\end{array}$ & $\bullet^{12}$ & \\
\hline & 99 LO CRK7 At4g23150 & CRK7 & & $e^{5}$ & - & - & $\bullet^{5}$ & - & $-8,12$ & - & 0 & 0 & - & & \\
\hline & $99 \longleftarrow$ CRK15 At4g23230 & CRK15 & & & - & - & & - & & & & & & & \\
\hline & ${ }_{98}$ — CRK10 At4g23180* & CRK10 & $\bullet 1$ & $\bullet 1$ & - & - & & - & -8 & - & 0 & 0 & - & & \\
\hline & 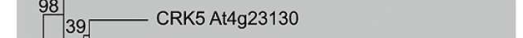 & CRK5 & $\bullet 1$ & $\bullet 1$ & - & - & & - & $\bullet^{8}$ & - & 0 & - & - & $\bullet^{13}$ & $\bullet^{2}$ \\
\hline & - CRK4 At3945860 & CRK4 & $\bullet^{2}$ & $\bullet^{2}$ & - & - & & - & $-8,12$ & - & - & - & - & $\bullet^{12}$ & $\bullet^{2}$ \\
\hline & $9 8 \longdiv { 9 3 } -$ CRK23 At4923310 & CRK23 & & & & & & - & $-8,12$ & - & - & - & - & -12 & \\
\hline & 95 CRK20 At4g23280 & CRK20 & $\bullet^{2}$ & $\bullet^{2}$ & - & - & & - & $\bullet^{8}$ & - & 0 & 0 & - & & $\bullet^{2}$ \\
\hline & 100- CRK19 At4923270 & CRK19 & $\bullet^{2}$ & $\bullet^{2}$ & - & - & & - & 8 & - & 0 & 0 & - & & $\bullet^{2}$ \\
\hline & L CRK25 At4905200 & CRK25 & & & & & & - & -8 & - & o & $\circ$ & - & & \\
\hline & Group IV — CRK22 At4g23300 & CRK22 & & & - & - & $\bullet^{10}$ & - & -8 & - & o & $\circ$ & - & & \\
\hline & 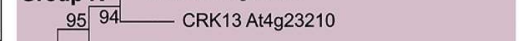 & CRK13 & & $\bullet^{6}$ & - & - & $\bullet 6,10$ & - & $-8,12$ & $\circ$ & o & $\circ$ & - & $\bullet^{6}$ & $\bullet^{6}$ \\
\hline & 43 CRK11 At4923190 & CRK11 & $\bullet 1$ & $\bullet 1,7$ & - & - & $\bullet{ }^{10}$ & - & -8 & - & - & $\circ$ & - & & \\
\hline & - CRK35 At4g 11500 putative pseudogene & CRK35 & & & - & - & & - & & & & & & & \\
\hline & $50 \sqrt{99}-$ CRK34 At4g11530 & CRK34 & & & - & - & & - & & & & & & & \\
\hline & CRK33 At4911490 & CRK33 & & & & & & - & -8 & - & o & - & - & & \\
\hline & 25 - CRK14 At4923220 & CRK14 & & & - & - & $\bullet^{10}$ & - & & & & & & & \\
\hline & 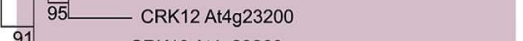 & CRK12 & & & & & & - & -8 & - & - & 0 & - & & \\
\hline & 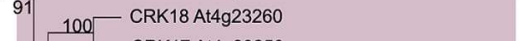 & CRK18 & & & - & - & $\bullet^{10}$ & - & & & & & & & \\
\hline & L CRK17 At4g23250 & CRK17 & $\bullet^{3}$ & & - & - & & - & 8 & - & 0 & $\circ$ & - & & \\
\hline & $100-$ CRK32 At4911480 & CRK32 & & & - & - & & - & -8 & - & - & - & - & & \\
\hline & $922_{36} \longrightarrow$ CRK31 At4911470 & CRK31 & & & - & - & & - & -8 & $\circ$ & $\circ$ & $\circ$ & - & & \\
\hline & CRK30 At4g 11460 & CRK30 & & & - & - & & - & & & & & & & \\
\hline & $83 \square$ CRK16 At4g23240 & CRK16 & & & - & - & & - & -8 & - & o & - & - & & \\
\hline & $100 \_$CRK24 At4g23320 & CRK24 & & & - & - & & - & 8 & - & 0 & o & - & & \\
\hline & $94 \_—$ CRK21 At4g23290 & CRK21 & & & o & o & & - & 8 & - & o & o & - & & \\
\hline & Group III $\ulcorner$ CRK39 At4904540 & CRK39 & & & - & - & & - & -8 & - & o & $\circ$ & - & & \\
\hline & $83^{100}$ CRK40 At4g04570 & CRK40 & & & - & - & & - & -8 & - & - & - & - & & \\
\hline & 91 L_ CRK38 At4904510 & CRK38 & & & - & - & & - & -8 & - & & - & - & & \\
\hline & 100 L CRK37 At4904500 & CRK37 & & & - & - & & - & $-8,12$ & - & - & - & - & -12 & \\
\hline & — CRK36 At4904490 & CRK36 & $\bullet^{4}$ & $\bullet^{4}$ & - & - & $\bullet^{4}$ & - & $\bullet^{4}$ & & & & & $\bullet 4,12$ & $\bullet 4$ \\
\hline & Group $\|_{100}$ CRK28 At4g21400 & CRK28 & & & - & - & $\bullet^{10}$ & - & $-10 / \bullet^{8}$ & - & 0 & 0 & - & $\bullet^{10}$ & $\bullet^{10}$ \\
\hline & 99 L CRK29 At4921410 & CRK29 & & & - & $\circ$ & $\bullet{ }^{10}$ & - & $-10 / \bullet^{8}$ & - & o & $\circ$ & - & & $\bullet{ }^{10}$ \\
\hline & CRK41 Attg00970 & CRK41 & & & $\bullet$ & - & & $\bullet$ & -8 & - & o & & - & & \\
\hline & 90 100 CRK44 A44900960 (no ED) & CRK44 & & & - & - & & - & & & & & & & \\
\hline & CRK26 At4938830 & CRK26 & & & - & - & & - & & & & & & & \\
\hline$\frac{1}{28}$ & \begin{tabular}{|l|l|l}
8 & 43 & CRK27 At4921230
\end{tabular} & CRK27 & & & - & - & & - & & & & & & & \\
\hline & CRK45 At4g11890 (no ED) & CRK45 & & & - & - & & - & 8 & - & 0 & 0 & - & & \\
\hline & $\begin{array}{l}47 \\
\quad \text { CRK43 At1970740 (no ED) }<\end{array}$ & CRK43 & & & - & - & & - & & & & & & & \\
\hline & CRK46 At4928670 & CRK46 & & & & & & - & -8 & - & - & - & - & & \\
\hline & 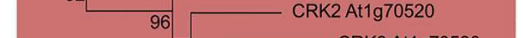 & CRK2 & & & - & - & & - & $-8 / \bullet 11$ & o & - & - & - & & \\
\hline & $\sqrt{56} \quad$ CRK3 At1970530 & CRK3 & & & - & - & & - & -8 & o & 0 & $\circ$ & - & & \\
\hline & CR1 At At1919090 & CRK1 & & & & & & - & -8 & - & - & $\circ$ & - & & \\
\hline 0.2 & Broup I $\quad 78-$ CRK42 At5g40380 & CRK42 & & & - & - & & - & -8 & - & 0 & o & - & & \\
\hline
\end{tabular}


Figure 3: Phylogenetic tree of all CRKs and overview of immunity-related CRK gene expression and mutant phenotypes.

A: Phylogenetic tree of all CRKs generated by alignment of the amino acid sequence of all Arabidopsis CRKs. The CRKs are grouped into five groups (different colors). CRKs lacking the ectodomain (ED) and transmembrane domain are highlighted with an arrowhead. CRKs marked with a white circle are topic of this study. Phylogenetic tree adapted from Bourdais et al., 2015. B: Overview of immunity-related CRK gene expression. Black dots represent transcriptional upregulation, open circles represent downregulation. C and D: Knockout/ knockdown (C) and overexpression (D) mutant phenotypes. Black dots represent enhanced phenotypes, open circles represent reduced phenotypes. B - D: “-“= investigated, but not significantly altered. SA= salicylic acid, Pst= Pseudomonas syringae pv. tomato DC 3000, Bgh= Blumeria graminis f.sp. hordeii, G. orontii $=$ Golovinomyces orontii, flg $22=$ flagellin $22 ; \mathrm{O}_{3}=$ ozone; ROS $=$ Reactive oxygen species. ${ }^{1}=\mathrm{Du}$ and Chen, 2000; ${ }^{2}=$ Chen et al., 2004; ${ }^{3}=$ Ohtake et al., 2000; ${ }^{4}=$ Lee et al., 2017; ${ }^{5}=$ Dinischiotu, $2011 ;{ }^{6}=$ Acharya et al, $2007 ;{ }^{7}=$ Czernic et al, $1999 ;{ }^{8}=$ Bourdais et al, 2015; ${ }^{9}=$ Wrzaczek et al, $2010 ;{ }^{10}=$ Yadeta et al, $2017 ;{ }^{11}=$ Kimura et al, $2020 ;{ }^{12}=$ Yeh et al, $2015 ;{ }^{13}=$ Chen et al, 2003. 
One of the tested treatments was ozone $\left(\mathrm{O}_{3}\right)$, which induces a ROS burst in the apoplast similar to the oxidative burst in plant-pathogen interactions. The transcription of $18 C R K$ s, remarkably including all members of subgroup three, was significantly induced in response to $\mathrm{O}_{3}$ (Wrzaczek et al., 2010). Several groups investigated the expression of selected CRKs upon treatment with the plant hormone salicylic acid and in summary nine $C R K$ s were reported as transcriptionally upregulated (Czernic et al., 1999; Du and Chen, 2000; Ohtake et al., 2000; Chen et al., 2004; Lee et al., 2017). In the same and additional studies, it was demonstrated that ten CRKs were transcriptionally enhanced in response to Pseudomonas syringae infection (Czernic et al., 1999, Du and Chen, 2000; Chen et al., 2004; Acharya et al., 2007; Dinischiotu, 2011; Lee et al., 2017) and flg22 treatment induced transcription of nine CRKs (Acharya et al., 2007; Dinischiotu, 2011; Lee et al., 2017; Yadeta et al., 2017). Infection with the non-adapted powdery mildew $B g h$ and the adapted powdery mildew Golovinomyces orontii (Go) significantly induced transcript abundance of $28 C R K \mathrm{~s}$ and 10 CRKs respectively (Figure 3B) (Bourdais et al., 2015).

Moreover, several CRKs including CRK4, CRK5, CRK7 and CRK8 were upregulated in bakl bkk1 (de Oliveira et al., 2016) as well as in bakl bikl double mutants (Liu et al., 2017b).

Taken together, these results suggest that CRKs might be involved in defense responses against bacterial and fungal pathogens.

To further investigate the function of Arabidopsis CRKs, Bourdais and colleagues (2015) genotyped T-DNA insertion lines for all CRKs and homozygous T-DNA insertion lines with no or a reduced transcript level could be obtained for 35 different CRKs. Several crk mutants exhibited slight developmental phenotypes as they showed delayed germination and/ or early senescence. Moreover, some $c r k$ mutants were more susceptible towards light and/ or salt stress. Most interestingly, many crk mutants showed altered immunity related phenotypes (Figure 3C). In 10 crk TDNA lines ROS production was significantly increased upon flg22 treatment whereas it was significantly decreased in four crk mutants, suggesting antagonistic roles for CRKs in ROS accumulation (Bourdais et al., 2015). Bourdais and colleagues observed that crk5, crk20, crk28 and crk29 mutant lines were significantly more susceptible to Pseudomonas syringe pv. tomato (Pst) infection compared to WT. In contrast to this, Yadeta and colleagues (2017) did not detect altered resistance of crk28 and crk29 towards Pst and Kimura and colleagues (2020) demonstrated higher Pst susceptibility of $c r k 2$. Additionally, bacterial titers were higher in crk36 plants upon Pst infection than in WT (Lee et al., 2017). Ten crk mutants showed enhanced susceptibility towards Golovinomyces orontii infection (Bourdais et al., 2015), suggesting that CRKs are involved in defense against both bacterial and fungal pathogens. 
As several pathogens enter the plant through stomata, stomatal closure is triggered upon PAMP perception to impede pathogen entry. Surprisingly, PAMP-triggered stomatal closure appears to be regulated by multiple CRKs, as 22 crk mutant lines showed reduced stomatal closure in response to flg22 treatment, including four mutant lines that were more susceptible towards infection with Pst. Stomatal closure in response to chitin was impaired in $21 \mathrm{crk}$ mutant lines and two lines showed enhanced stomatal closure (Bourdais et al., 2015).

In summary, the analysis of a collection of crk T-DNA mutants revealed that CRKs are involved in developmental processes, in response to abiotic stresses but predominantly appear to fulfill important functions for plant immunity. Nevertheless, most crk mutants were lacking strong phenotypes, very likely due to functional redundancies among closely related CRKs (Bourdais et al., 2015).

To overcome this limitation and to investigate possible CRK functions in immunity in more detail, phenotypes of CRK overexpressing lines were examined (Figure 3D). Overexpression of CRK4 (Yeh et al., 2015), CRK5 (Chen et al., 2003), CRK6 (Yeh et al., 2015), CRK13 (Acharya et al., 2007), CRK28 (Yadeta et al., 2017) and CRK36 (Yeh et al., 2015; Lee et al., 2017) led to enhanced resistance towards Pst infection, suggesting a positive involvement of these CRKs in defense against bacterial pathogens. Additionally, overexpression of five of these CRKs (CRK4, CRK5, CRK13, CRK19 and CRK20) induced cell death in uninfected Arabidopsis plants (Chen et al., 2004; Acharya et al., 2007), suggesting that the enhanced resistance against Pst in these lines is caused by an upregulated cell death response. Transient expression of CRK13, CRK28 and CRK29 induced rapid and massive cell death in $N$. benthamiana and CRK36 overexpressing plants showed an early senescence phenotype and enhanced cell death after treatment with Pst or the fungal toxin fumonisin B (Lee et al., 2017). CRK5-induced programmed cell death was shown to be SA-, NPR1-, EDS1 and NDR1 independent (Chen et al., 2003), whereas CRK13 induced cell death was SA dependent (Acharya et al., 2007). Taken together, these results indicate that CRKs are involved in age-dependent, pathogen induced and toxin-triggered cell death formation. Four of the eight CRKs that trigger cell death when overexpressed belong to subfamily five and two to subfamily two. However, the effect of CRK overexpression was not investigated for all CRKs yet. Therefore, the ability to induce age-dependent, pathogen induced and/or toxin-triggered cell death currently cannot be assigned to any CRK-subfamily.

Since CRKs have a kinase domain that might be important for signal transduction, it was investigated if kinase activity of CRK5, CRK28 and CRK36 is required for cell death induction. Overexpression of kinase dead CRK5, CRK28 and CRK36 mutant versions did not induce cell death formation, concluding that indeed a functional kinase domain is required (Chen et al., 2003; Lee et al., 
2017; Yadeta et al., 2017). Similarly, overexpression of mutants lacking one of the conserved cysteine residues in the CRK28 ectodomain or all conserved cysteine residues in one of the CRK36 DUF26 domains did not lead to cell death, indicating that the conserved cysteine residues were necessary for functions of these CRKs (Lee et al., 2017; Yadeta et al., 2017).

A rice CRK, that is similar to Arabidopsis subgroup one CRKs with regard to the cysteines in the second DUF26 domain, was also shown to be involved in cell death formation. In this CRK, named APOPTOSIS LEAF AND SHEATH 1 (ALS1) or LIGHT-INDUCED LESION MIMIC MUTANT 1 (LIL1), a single amino acid exchange of valine ${ }^{429}$ to isoleucine (Zhou et al., 2017) or leucine (Du et al., 2019) in the kinase domain resulted in the development of light-induced spontaneous lesions (Zhou et al., 2017; Du et al., 2019). Interestingly, the enhanced lesion formation in als 1/ lill amino acid mutants was semi-dominant. Moreover, untreated als1/ lill mutant plants accumulated ROS, chloroplasts were destructed, defense gene expression was highly induced and resistance to the rice blast fungus Magnaporthe grisea was enhanced (Zhou et al., 2017; Du et al., 2019). ALS1/ LIL1 expression was strongly induced in the mutant plants once lesions started to appear (Zhou et al., 2017; Du et al., 2019). Overexpression of WT ALS1/ LIL1 also triggered enhanced lesion formation and the severity of lesions correlated with $A L S 1 / L I L 1$ expression levels. Therefore it was suggested that the robust HR-like cell death is due to ALS1/ LIL1 overexpression in a dose-dependent manner (Du et al., 2019).

For some Arabidopsis CRKs interaction with immune receptors was demonstrated. One example for such an interaction is CRK28. CRK28 and the closely related CRK29 are among the kinases that mediate cell death in $N$. benthamiana (Yadeta et al., 2017). Moreover, CRK28 was shown to interact with BAK1 and FLS2 in an flg22-independent manner and the $N$. benthamiana BAK1 homologue $N b$ SERK3 was required for CRK28-induced cell death formation in $N$. benthamiana. Additionally, Co-IP experiments demonstrated that CRK28 self-associates and interacts with CRK29 in an flg22 independent manner suggesting the formation of a receptor complex (Yadeta et al., 2017). Interestingly, bacterial titers in crk22, crk28 and crk29 single knockdown or knockout lines were not altered upon Pst infection compared to WT, but bacterial growth was significantly enhanced in a triple mutant line, indicating redundant functions of these CRKs (Yadeta et al., 2017).

In other studies, CRK4, CRK6 and CRK36 were shown to associate with FLS2 independently of flg22 elicitation in Arabidopsis protoplasts (Yeh et al., 2015; Lee et al., 2017). Additionally it was demonstrated that CRK36 interacts with BIK1 in untreated plants and slightly stronger in flg22 treated plants (Lee et al., 2017). A BIK1 band shift in Western Blot upon flg22 treatment was visible in WT and CRK36 overexpressing plants, but absent in plants overexpressing CRK36 mutant 
versions in which all cysteine residues either of the first or the second DUF26 domain are mutated to alanine. This suggests that CRK36 is involved in BIK1 phosphorylation and that the conserved cysteine residues are required for this function (Lee et al., 2017). CRK36 overexpression lines show enhanced flg22 and Pst triggered stomatal closure, are defective in stomatal re-opening upon Pst infection and accordingly resistance towards Pst is enhanced (Lee et al., 2017). These phenotypes were impaired in CRK36OE bikl plants and CRK36OE rbohD/F mutants lost both flg22 induced stomatal closing and resistance to Pst, demonstrating that BIK1, RBOHD and RBOHF are required for CRK36 function in stomatal immunity (Lee et al., 2017). Lee and colleagues proposed a model in which CRK36 is activated by sensing ROS through redox modifications of cysteine residues leading to BIK1 phosphorylation. Activated BIK1 is known to phosphorylate RBOHD/F leading to further ROS production. Therefore, CRK36 is suggested to function as a ROS receptor and to participate in a positive feedback loop together with BIK1, RBOHD/F and ROS (Lee et al., 2017).

Additionally to the interaction with immune receptors, CRK36 was able to homodimerize in yeasttwo hybrid assays and interaction with CRK45 was demonstrated by bimolecular fluorescence complementation and co-IP (Tanaka et al., 2012). CRK45 is one of three Arabidopsis CRCKs lacking an ectodomain (Vaattovaara et al., 2019). CRK36 and CRK45 knockdown lines were more sensitive to high-salinity, osmotic stresses and ABA treatment than WT plants, suggesting that CRK36 is not only involved in plant immunity processes (Tanaka et al., 2012).

Another example for a CRK that interacts with plant immune system components is CRK2, which was shown to be involved in flg22-induced ROS production, stomatal closure, $\mathrm{Ca}^{2+}$ accumulation and MAPK activation (Kimura et al., 2020). Co-immunoprecipitation experiments revealed that CRK2 interacts with RBOHD independently of flg22 treatment. In vitro kinase assay experiments and mass spectrometry analysis unveiled that CRK2 directly phosphorylates RBOHD at several Nand C-terminal residues. Some of these sites were confirmed to be phosphorylated upon flg22 treatment in planta. CRK2 is at least partially responsible for phosphorylation of one site (S703) as its phosphorylation in RBOHD was significantly reduced in CRK2 kinase dead plants. Moreover, RBOHDS703A mutants showed reduced ROS production and stomatal closure upon flg22 perception resulting in enhanced susceptibility towards Pst (Kimura et al., 2020).

In summary, it was shown that CRKs are able to homodimerize and to heterodimerize with other CRKs or CRCK as well as with receptors and cytoplasmic kinases involved in plant immune responses and proteins necessary for ROS accumulation. This makes the CRK family an important class of RLKs involved in immunity, potentially as receptors, co-receptors or helper receptors. To date it is still unknown, which ligand or class of ligand CRKs might bind. One hypothesis is that 
CRKs might bind carbohydrates since mannose-binding was demonstrated for other DUF26 domain containing proteins (Miyaka et al., 2014; Ma et al., 2018). Nevertheless, the mannose binding residues are not conserved among Arabidopsis CRKs (Figure S1A), suggesting that CRKs bind a different ligand.

Two Arabidopsis CRKs, CRK7 and CRK43, are the main topics of this study. Therefore, all available information on these CRKs will be discussed below.

CRK43 is one of the Arabidopsis CRCKs lacking an ectodomain. Very little is known about CRK43 to date. The only published data come from large-scale transcriptional analyses, which indicate that $C R K 43$ expression is upregulated upon $\mathrm{O}_{3}$ treatment and $B g h$ infection (Wrzaczek et al., 2010; Bourdais et al., 2015).

More information is available for CRK7. Expression of CRK7 was induced upon Pst, Botrytis cinerea and Bgh infection (Wrzaczek et al., 2010; Dinischiotu, 2011; Bourdais et al., 2015), as well as after $\mathrm{O}_{3}$ and flg22 treatment (Wrzaczek et al., 2010; Dinischiotu, 2011; Idänheimo et al., 2014). Moreover, $C R K 7$ expression was highly increased upon treatment with the oomycete-derived elicitor NECROSIS-INDUCING PHYTOPHTORA PROTEIN 1 (NPP1), a cell wall protein that triggers defense responses in Arabidopsis (Fellbrich et al., 2002; Winter et al., 2007) as well as upon treatment with the bacterial-derived elicitor HarpinZ (HrpZ), which is highly secreted through the type-III-secretion system by $P$. syringae, probably targets the host plasma membrane and induces cell death in Arabidopsis (Alfano and Collmer, 1997; Haapalainen et al., 2012; Winter et al., 2007). Furthermore $C R K 7$ expression was highly induced in a line overexpressing LecRK-VI.2, a protein kinase associated with FLS2 and critical for Arabidopsis resistance to bacterial pathogens (Singh et al., 2012; Huang et al., 2014; Yeh et al., 2015). CRK7 expression was also induced in bik1 and bakl single mutants and more than 100-fold induced in bakl bikl double mutants (Liu et al., 2017b). In bakl bkk1 mutants CRK7 expression was upregulated as well (de Oliveira et al., 2016). In contrast, reduced $C R K 7$ expression was observed under heat and light stress conditions (Wrzaczek et al., 2010; Dinischiotu, 2011).

Knockout of crk7 did not alter resistance towards Pst and B. cinerea as well as callose deposition upon flg22 and elf1 8 treatment, but resulted in stronger accumulation of $\mathrm{H}_{2} \mathrm{O}_{2}$ upon $\mathrm{O}_{3}$ treatment (Dinischiotu, 2011; Idänheimo et al., 2014; Yeh et al., 2015). A line silenced for the closely related CRKs CRK6, CRK7, CRK8, CRK10 and CRK15 developed stronger cell death in response to $\mathrm{O}_{3}$ than the single mutants, suggesting functional redundancies between these CRKs (Idänheimo et al., 2014). 
Yeh and colleagues (2015) could not obtain a CRK7 overexpressing line and hypothesized that this is due to lethality of CRK7 overexpression similar to CRK13 overexpression. Nevertheless, Idänheimo and colleagues managed to isolate CRK7 overexpression lines and showed that $\mathrm{O}_{3}$ tolerance is not altered in those.

Moreover, CRK7 kinase activity was demonstrated, as CRK7 was able to auto-phosphorylate and to phosphorylate the artificial substrate MBP in vitro (Idänheimo et al., 2014).

In the work of Dinischiotu (2011), CRK7 was identified as responsible for chlorosis and necrosis induction in Arabidopsis in response to infiltration with Staphylococcus epidermidis, a gram positive bacterium that colonizes skin and mucous membranes of mammals but cannot grow on Arabidopsis. The observed cell death phenotype was also present upon treatment with boiled S. epidermidis suspensions, suggesting that Arabidopsis recognizes a heat stable elicitor for cell death induction. The exact nature of this S. epidermidis substance is not known, but multiple biochemical assays suggest that it is a polysaccharide (Dinischiotu, 2011). This indicates that CRKs may bind polysaccharides similar to the mannose binding activity observed for CRRSPs.

\subsection{Thesis aims}

The LysM RLK Chitin Elicitor Receptor Kinase 1 (CERK1) is a major component of the Arabidopsis chitin receptor (Miya et al., 2007). cerk1-4 is a mutant allele with an amino acid exchange in the extracellular domain and cerkl-4 plants show an increased SA-dependent deregulated cell death response mediated by the CERK1-4 N-terminal domain alone (Petutschnig et al., 2014). CERK1 undergoes ectodomain shedding resulting in a soluble N-terminal fragment in Western Blots. This $33 \mathrm{kDa}$ fragment is probably still cleaved in CERK1-4 but unstable as it is not visible in Western blots (Petutschnig et al., 2014).

It is yet unclear how the ectodomain is shed and if the shed ectodomain, the lack of the shed ectodomain or turnover products are perceived by an unknown receptor. Moreover it is unclear how recognition or the lack of recognition induces or prevents cerk1-4 mediated cell death. It is also unclear how SA accumulates in cerk1-4 plants to a high level and, finally, how cell death is induced. To identify molecular components necessary for cerk1-4 cell death induction, an ethyl methanesulfonate (EMS) treated Arabidopsis cerk1-4 mutant population was generated and used for a cell death suppressor screen (M. Stolze and E. Petutschnig, unpublished). In this screen, the extralarge G protein XLG2 was identified as a molecular component for cerk1-4 cell death induction (M. Stolze, unpublished; Meusel, 2016). 
The aim of this work was to identify and functionally characterize additional molecular components from the cerkl-4 suppressor screen using a mutation mapping strategy based on nextgeneration sequencing. Three independent mutants were successfully isolated, underlying mutated candidate genes mapped and identified as the novel cerkl-4 suppressors CRK7 and CRK43 as well as the already known suppressor PAD4. Thus, further work focused on the two CRKs. It was the aim to confirm their involvement in cerkl-4 cell death induction by analysis of independent mutant lines and complementation studies. Moreover, the proteins should be characterized with regard to cellular localization, interaction with other proteins and enzymatic activity to get insights into their biological function and involvement in cerk1-4 dependent deregulated cell death execution. 


\section{Materials and methods}

\section{$\underline{\text { 2.1 Materials }}$}

\subsubsection{Plants}

\subsubsection{Arabidopsis thaliana}

The Arabidopsis (L.) Heynh. accessions Columbia-0 (Col-0) and Col-3 gll were used as wild type lines. T-DNA insertion lines from the SALK collection (Alonso et al., 2003) were ordered from the Nottingham Arabidopsis Stock Center (NASC). T-DNA insertion lines from the GABI collection were obtained from GABI-KAT (Kleinboelting et al., 2012). Information on T-DNA mutants (Table 1), EMS mutants (Table 2) and double mutant lines (Table 3) used and/ or generated in this work are listed below. Transgenic lines generated and used in this work are listed in table 4.

Table 1: Arabidopsis thaliana single T-DNA mutant lines used in this work.

\begin{tabular}{lllll}
\hline Allele & AGI locus & Background & T-DNA & Reference \\
\hline cerk1-2 & At3g21630 & Col-0 & GABI_096F09 & Miya et al., 2007 \\
$f l s 2 c$ & At5g46330 & Col-0 & SAIL_691_C4 & Cyril Zipfel \\
crk7-5 & At4g23150 & Col-0 & GABI_643A06 & This work \\
crk43-1 & At1g70740 & Col-0 & SALK_091320 & This work \\
crk43-3 & At1g70740 & Col-0 & SALK_201662 & This work \\
\end{tabular}

Table 2: Arabidopsis thaliana single EMS mutant lines used in this work.

\begin{tabular}{lllll}
\hline Allele & AGI locus & Background & Mutation type & Reference \\
\hline cerk1-4 & At3g21630 & Col-3 gll & EMS & $\begin{array}{l}\text { Petutschnig } \text { et al., } \\
\text { crk7-4 }\end{array}$ \\
At4g23150 & Col-3 gll & EMS & This work \\
crk43-2 & At1g70740 & Col-3 gll & EMS & This work \\
\hline
\end{tabular}


Table 3: Arabidopsis thaliana double mutant lines isolated/generated in this work.

\begin{tabular}{|c|c|c|c|c|}
\hline Allele & AGI locus & Background & Mutation type & Reference \\
\hline cerk1-4 crk7-4 & $\begin{array}{l}\text { At3g21630 } \\
\text { At4g23150 }\end{array}$ & Col-3 gll & EMS & This work \\
\hline cerk1-4 crk7-6 & $\begin{array}{l}\text { At3g21630 } \\
\text { At4g23150 }\end{array}$ & Col-3 gll & EMS & This work \\
\hline cerk1-4 crk7-7 & $\begin{array}{l}\text { At3g21630 } \\
\text { At4g23150 }\end{array}$ & Col-3 gll & EMS, CRISPR/ Cas9 & This work \\
\hline cerk1-4 crk7-8 & $\begin{array}{l}\text { At3g21630 } \\
\text { At4g23150 }\end{array}$ & Col-3 gll & EMS, CRISPR/ Cas9 & This work \\
\hline $\begin{array}{l}\text { cerk1-4 crk43-1 } \\
\text { (het/ hom) }\end{array}$ & $\begin{array}{l}\text { At3g21630 } \\
\text { At1g70740 }\end{array}$ & Col-3 gll & EMS, T-DNA & This work \\
\hline cerk1-4 crk43-2 & $\begin{array}{l}\text { At3g21630 } \\
\text { At1g70740 }\end{array}$ & Col-3 gll & EMS & This work \\
\hline cerkl-4 crk43-3 & $\begin{array}{l}\text { At3g21630 } \\
\text { At1g70740 }\end{array}$ & Col-3 gll & EMS, T-DNA & This work \\
\hline cerk1-4 pad4-14 & $\begin{array}{l}\text { At3g21630 } \\
\text { At3g52430 }\end{array}$ & Col-3 gll & EMS & This work \\
\hline
\end{tabular}

Table 4: Transgenic Arabidopsis thaliana lines generated in this work.

\begin{tabular}{|c|c|c|c|c|}
\hline Transgene & Background & Vector & Selection marker & Reference \\
\hline CRK7 & cerk1-4 crk7-4 & $\begin{array}{l}\text { pGreenII-0229- } \\
\text { pCRK7::CRK7 }\end{array}$ & Basta $^{\circledR R}$ & This work \\
\hline CRK7 & cerk1-4 crk7-4 & $\begin{array}{l}\text { pGreenII-Kan- } \\
\text { p35S::CRK7 }\end{array}$ & $\mathrm{Kan}^{\mathrm{R}}$ & This work \\
\hline CRK7 & cerk1-4 crk7-5 & $\begin{array}{l}\text { pGreenII-Kan- } \\
\text { p35S::CRK7 }\end{array}$ & $\mathrm{Kan}^{\mathrm{R}}$ & This work \\
\hline CRK7-4 & cerkl-4 & $\begin{array}{l}\text { pGreenII-0229- } \\
\text { pCRK7::crk7-4 }\end{array}$ & Basta $^{\circledR R}$ & This work \\
\hline CRK7-4 & cerkl-4 & $\begin{array}{l}\text { pGreenII-Kan- } \\
\text { p35S::crk7-4 }\end{array}$ & $\mathrm{Kan}^{\mathrm{R}}$ & This work \\
\hline CRK7-Citrine & cerk1-4 crk7-4 & $\begin{array}{l}\text { pGreenII-0229- } \\
\text { pCRK7::CRK7- } \\
\text { Citrine }\end{array}$ & Basta $^{\circledR R}$ & This work \\
\hline
\end{tabular}




\begin{tabular}{|c|c|c|c|c|}
\hline CRK7-Citrine & cerk1-4 crk7-4 & $\begin{array}{l}\text { pGreenII-0229- } \\
\text { p35S::CRK7-Citrine }\end{array}$ & Basta $^{\circledR R}$ & This work \\
\hline CRK7-Citrine & cerk1-4 crk7-5 & $\begin{array}{l}\text { pGreenII-0229- } \\
\text { p35S::CRK7-Citrine }\end{array}$ & Basta $^{\circledR R}$ & This work \\
\hline CRK7-Citrine & cerkl-4 & $\begin{array}{l}\text { pGreenII-0229- } \\
\text { p35S::CRK7-Citrine }\end{array}$ & Basta $^{\circledR R}$ & This work \\
\hline CRK7-FLAG & cerk1-4 crk7-5 & $\begin{array}{l}\text { pGreenII-0229- } \\
\text { pCRK7::CRK7-FLAG }\end{array}$ & Basta $^{\circledR R}$ & This work \\
\hline CRK7-FLAG & cerk1-4 crk7-5 & $\begin{array}{l}\text { pGreenII-0229- } \\
\text { p35S::CRK7-FLAG }\end{array}$ & Basta $^{\circledR R}$ & This work \\
\hline CRK7-mCitrine & Col-3 gll & $\begin{array}{l}p \text { GreenII-0229- } \\
\text { pCRK7::CRK7-mCitrine }\end{array}$ & Basta $^{\circledR R}$ & This work \\
\hline CRK7-mCitrine & Col-3 gll & $\begin{array}{l}\text { pGreenII-0229- } \\
\text { p35S::CRK7-mCitrine }\end{array}$ & Basta $^{\circledR R}$ & This work \\
\hline CRK7-4-Citrine & cerkl-4 & $\begin{array}{l}\text { pGreenII-0229- } \\
\text { pCRK7::crk7-4-Citrine }\end{array}$ & Basta $^{\circledR R}$ & This work \\
\hline CRK7-4-Citrine & cerkl-4 & $\begin{array}{l}\text { pGreenII-0229- } \\
\text { p35S::crk7-4-Citrine }\end{array}$ & Basta $^{\circledR R}$ & This work \\
\hline CRK7-4-mCitrine & Col-3 gll & $\begin{array}{l}\text { pGreenII-0229- } \\
\text { pCRK7::crk7-4-mCitrine }\end{array}$ & Basta $^{\circledR R}$ & This work \\
\hline CRK7-4-mCitrine & Col-3 gll & $\begin{array}{l}\text { pGreenII-0229- } \\
\text { p35S::crk7-4-mCitrine }\end{array}$ & Basta $^{\circledR R}$ & This work \\
\hline CRK43 & cerkl-4 crk43-2 & $\begin{array}{l}p \text { GreenII-0229- } \\
\text { pCRK43::CRK43 }\end{array}$ & Basta $^{\circledR R}$ & This work \\
\hline CRK43-mCitrine & cerkl-4 crk43-2 & $\begin{array}{l}\text { pGreenII-0229- } \\
\text { pCRK43::CRK43- } \\
\text { mCitrine }\end{array}$ & Basta $^{\circledR R}$ & This work \\
\hline
\end{tabular}

\subsubsection{Nicotiana benthamiana}

$N$. benthamiana seeds were originally obtained from T. Romeis (Biochemistry of Plants, Institute of Biology, Freie Universität Berlin). Around five-week-old N. benthamiana plants were used for transient expression mediated by Agrobacterium tumefaciens.

\subsubsection{Fungal pathogens}

\subsubsection{Blumeria graminis f.sp. hordei}

The non-adapted filamentous powdery mildew Blumeria graminis f.sp. hordei (Bgh; Lipka et al., 2005) was used for inoculation experiments of Arabidopsis plants. 


\subsubsection{Erysiphe cruciferarum}

The adapted powdery mildew Erysiphe cruciferarum (E. cruciferarum; Ec) was used for inoculation experiments of Arabidopsis plants. E. cruciferarum was originally obtained from the Max-Planck-Institute for Plant Breeding Research, Cologne, Germany.

\section{$\underline{2.1 .3 \text { Bacterial strains }}$}

\subsubsection{Escherichia coli}

Chemically competent Escherichia coli (E.coli) TOP10 cells (Invitrogen ${ }^{\mathrm{TM}}$, Carlsbad, USA)

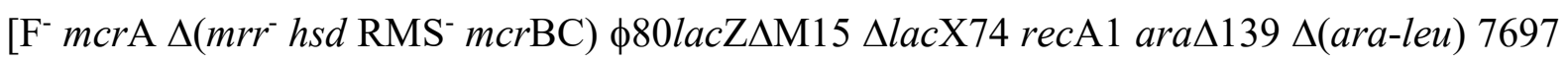
gal $\mathrm{U}$ gal $\mathrm{K} \operatorname{rps} \mathrm{L}\left(\mathrm{Str}^{\mathrm{R}}\right)$ end $\mathrm{A} 1$ nup $\left.\mathrm{G}\right]$ were used for cloning.

\subsubsection{Agrobacterium tumefaciens}

The A. tumefaciens strain GV3101 (Koncz and Schell, 1986) was used for transient expression in $N$. benthamiana leaves and stable transformation of $A$. thaliana plants. Three different strains were used that carry the helper plasmids pMP90, pMP90RK or pSoup (Koncz and Schell, 1986; Hellens et al., 2000).

\section{$\underline{2.1 .4 \text { Vectors }}$}

The following table lists vectors used or generated during this work, as well as their description and antibiotic resistance.

Table 5: Vectors used or generated in this study.

\begin{tabular}{|c|c|c|c|}
\hline Name & Description & $\begin{array}{l}\text { Selectionmarker } \\
\text { for bacteria and } \\
\text { plants }\end{array}$ & $\begin{array}{l}\text { Reference/ } \\
\text { Source }\end{array}$ \\
\hline pGreenII-0229 & $\begin{array}{l}\text { Binary vector for } A \text {. tumefaciens- } \\
\text { mediated transformation of plants }\end{array}$ & Kan $^{\mathrm{R}}$, Basta ${ }^{\circledR R}$ & $\begin{array}{l}\text { Hellens et al., } \\
2000\end{array}$ \\
\hline pGreenII-Kan & $\begin{array}{l}\text { Binary vector for } A \text {. tumefaciens- } \\
\text { mediated transformation of plants }\end{array}$ & $\mathrm{Kan}^{\mathrm{R}}, \mathrm{Kan}^{\mathrm{R}}$ & $\begin{array}{l}\text { Sieglinde } \\
\text { Kamrad }\end{array}$ \\
\hline $\begin{array}{l}p B-C R I S P R \text {-seed } G F P- \\
E E\end{array}$ & $\begin{array}{l}\text { Binary vector that is used as } \\
\text { Backbone for cloning of CRISPR } \\
\text { constructs and expresses Cas } 9 \\
\text { under an egg-cell specific promo- } \\
\text { tor. }\end{array}$ & $\mathrm{Spec}^{\mathrm{R}}$ & $\begin{array}{l}\text { Corinna } \\
\text { Thurow }\end{array}$ \\
\hline
\end{tabular}




\begin{tabular}{|c|c|c|c|}
\hline$E R$-ck & $\begin{array}{l}\text { Binary vector for } \text { A. tumefaciens- } \\
\text { mediated transformation of } \mathrm{N} \text {. } \\
\text { benthamiana and expression of } \\
\text { ER-ck. }\end{array}$ & $\operatorname{Kan}^{\mathrm{R}}$ & $\begin{array}{l}\text { Nelson et al., } \\
2007\end{array}$ \\
\hline $\begin{array}{l}\text { pC1-pUBQ10:: } \\
\text { mKate2-SYP122 }\end{array}$ & $\begin{array}{l}\text { Binary vector for } A \text {. tumefaciens- } \\
\text { mediated transformation of } \mathrm{N} \text {. } \\
\text { benthamiana and expression of } \\
\text { SYP122 with an N-terminal } \\
\text { mKate2-tag. }\end{array}$ & $\operatorname{Kan}^{\mathrm{R}}$ & $\begin{array}{l}\text { Elena } \\
\text { Petutschnig }\end{array}$ \\
\hline $\begin{array}{l}p C 1-p U B Q 10:: \\
m \text { Kate2-N7 }\end{array}$ & $\begin{array}{l}\text { Binary vector for } A \text {. tumefaciens- } \\
\text { mediated transformation of } \mathrm{N} \text {. } \\
\text { benthamiana and expression of } \\
\text { mKate } 2 \text { with a C-terminal nuclear } \\
\text { localization signal. }\end{array}$ & $\operatorname{Kan}^{\mathrm{R}}$ & $\begin{array}{l}\text { Hassan } \\
\text { Ghareeb }\end{array}$ \\
\hline $\begin{array}{l}\text { pGreenII-0229- } \\
\text { pCRK7::CRK7 } \\
\text { (pCT6) }\end{array}$ & $\begin{array}{l}\text { Binary vector for } A \text {. tumefaciens- } \\
\text { mediated transformation of plants } \\
\text { and expression of the CRK } \\
\text { gDNA under control of } p C R K 7 \text {. }\end{array}$ & Kan $^{\mathrm{R}}$, Basta ${ }^{\circledR R}$ & This work \\
\hline $\begin{array}{l}\text { pGreenII-Kan- } \\
\text { p35S::CRK7 } \\
\text { (pCT14) }\end{array}$ & $\begin{array}{l}\text { Binary vector for } A \text {. tumefaciens- } \\
\text { mediated transformation of plants } \\
\text { and expression of the CRK7 } \\
\text { gDNA under control of } p 35 S \text {. }\end{array}$ & $\operatorname{Kan}^{\mathrm{R}}, \operatorname{Kan}^{\mathrm{R}}$ & This work \\
\hline $\begin{array}{l}\text { pGreenII-0229- } \\
\text { pCRK7::crk7-4 } \\
\text { (pCT5) }\end{array}$ & $\begin{array}{l}\text { Binary vector for } A \text {. tumefaciens- } \\
\text { mediated transformation of plants } \\
\text { and expression of the crk7-4 } \\
\text { gDNA under control of } p C R K 7 \text {. }\end{array}$ & Kan $^{\mathrm{R}}$, Basta ${ }^{\circledR R}$ & This work \\
\hline $\begin{array}{l}\text { pGreenII-Kan- } \\
\text { p35S::crk7-4 } \\
\text { (pCT13) }\end{array}$ & $\begin{array}{l}\text { Binary vector for } A \text {. tumefaciens- } \\
\text { mediated transformation of plants } \\
\text { and expression of the crk7-4 } \\
\text { gDNA under control of } p 35 S \text {. }\end{array}$ & $\operatorname{Kan}^{\mathrm{R}}, \operatorname{Kan}^{\mathrm{R}}$ & This work \\
\hline $\begin{array}{l}p \text { GreenII-0229- } \\
\text { pCRK7::CRK7- } \\
\text { Citrine } e^{\mathrm{a}} \\
\text { (pCT2) }\end{array}$ & $\begin{array}{l}\text { Binary vector for } A \text {. tumefaciens- } \\
\text { mediated transformation of plants } \\
\text { and expression of the CRK7 } \\
\text { gDNA with a C-terminal Citrine- } \\
\text { tag under control of } p C R K 7 \text {. }\end{array}$ & $\operatorname{Kan}^{\mathrm{R}}$, Basta ${ }^{\circledR R}$ & This work \\
\hline $\begin{array}{l}\text { pGreenII-0229- } \\
\text { p35S::CRK7-Citrine } \\
(\text { pCT12) }\end{array}$ & $\begin{array}{l}\text { Binary vector for } A \text {. tumefaciens- } \\
\text { mediated transformation of plants } \\
\text { and expression of the CRK7 } \\
\text { gDNA with a C-terminal Citrine- } \\
\text { tag under control of } p 35 S \text {. }\end{array}$ & $\operatorname{Kan}^{\mathrm{R}}$, Basta ${ }^{\mathbb{R}}$ & This work \\
\hline $\begin{array}{l}\text { pGreenII-0229- } \\
\text { pCRK7::CRK7-FLAG } \\
(\mathrm{pCT} 20)\end{array}$ & $\begin{array}{l}\text { Binary vector for } A \text {. tumefaciens- } \\
\text { mediated transformation of plants } \\
\text { and expression of the CRK7 } \\
\text { gDNA with a C-terminal } \\
\text { 3xFLAG-tag under control of } \\
\text { pCRK7. }\end{array}$ & $\operatorname{Kan}^{\mathrm{R}}$, Basta ${ }^{\circledR R}$ & This work \\
\hline
\end{tabular}




\begin{tabular}{ll}
\hline$p$ GreenII-0229- & Binary vector for A. tumefaciens- \\
p35S::CRK7-FLAG & mediated transformation of plants \\
$($ pCT22) & and expression of the CRK7 \\
& gDNA with a C-terminal \\
& 3xFLAG-tag under control of \\
& $p 35 S$.
\end{tabular}

pGreenII-0229-

pCRK7::CRK7-

mCitrine ${ }^{\mathrm{d}}$

(pCT47)

pGreenII-0229-

p35S::CRK7-mCitrine ${ }^{\mathrm{d}}$ (pCT49)

pGreenII-0229-

p35S::crk7-4-Citrine

(pCT11)

pGreenII-0229-

pCRK7::crk7-4-

mCitrine ${ }^{\mathrm{d}}$

(pCT46)

pGreenII-0229p35S::crk7-4-mCitrine $\mathrm{d}^{\mathrm{d}}$ (pCT48)

pB-crispr-seed GFP$E E-C R K 7^{A+D}$

(pCT16)

pB-crispr-seedGFP$E E-C R K 7^{A+E}$

(pCT15)

pB-crispr-seed GFP$E E-C R K 7^{A+F}$ (pCT10)

Binary vector for A. tumefaciensmediated transformation of plants and expression of the CRK7 gDNA with a C-terminal mCitrine-tag under control of $p C R K 7$.

Binary vector for A. tumefaciensmediated transformation of plants and expression of the CRK7 gDNA with a C-terminal mCitrine-tag under control of p35S.

Binary vector for A. tumefaciensmediated transformation of plants and expression of the crk7-4 gDNA with a C-terminal Citrinetag under control of $p 35 S$.

Binary vector for A. tumefaciensmediated transformation of plants and expression of the crk7-4 gDNA with a C-terminal mCitrine-tag under control of $p C R K 7$.

Binary vector for A. tumefaciensmediated transformation of plants and expression of the crk7-4 gDNA with a C-terminal mCitrine-tag under control of p35S.

Binary vector for A. tumefaciensmediated transformation of plants for deletion of CRK7 via CRISPR/ Cas9. Binary vector for $A$. tumefaciensmediated transformation of plants for deletion of CRK7 via CRISPR/ Cas9.

Binary vector for A. tumefaciensmediated transformation of plants for deletion of CRK7 via
Kan $^{\mathrm{R}}$, Basta ${ }^{\mathrm{BR}} \quad$ This work

$\mathrm{Kan}^{\mathrm{R}}$, Basta ${ }^{\circledR \mathrm{R}} \quad$ This work

$\operatorname{Kan}^{\mathrm{R}}$, Basta ${ }^{\circledR R} \quad$ This work

Kan $^{\mathrm{R}}$, Basta ${ }^{\circledR R} \quad$ This work

Kan $^{\mathrm{R}}$, Basta ${ }^{\circledR R} \quad$ This work

Kan $^{\mathrm{R}}$, Basta ${ }^{\circledR R} \quad$ This work

Spec $^{\mathrm{R}}$, Basta ${ }^{\circledR R}, \quad$ This work seedGFP

Spec $^{\mathrm{R}}$, Basta ${ }^{\circledR R}, \quad$ This work CRISPR/ Cas9.

\section{seedGFP}

$\mathrm{Spec}^{\mathrm{R}}, \mathrm{Basta}^{\circledR R}$ seedGFP
This work 


\begin{tabular}{|c|c|c|c|}
\hline $\begin{array}{l}\text { pB-crispr-seed GFP- } \\
E E-C R K 7^{B+D} \\
\text { (pCT9) }\end{array}$ & $\begin{array}{l}\text { Binary vector for } A \text {. tumefaciens- } \\
\text { mediated transformation of plants } \\
\text { for deletion of } C R K 7 \text { via } \\
\text { CRISPR/ Cas } 9 .\end{array}$ & $\begin{array}{l}\text { Spec }^{\mathrm{R}}, \text { Basta }^{\mathbb{R R}} \text {, } \\
\text { seedGFP }\end{array}$ & This work \\
\hline $\begin{array}{l}\text { pB-crispr-seed GFP- } \\
E E-C R K 7^{B+E} \\
\text { (pCT8) }\end{array}$ & $\begin{array}{l}\text { Binary vector for } A \text {. tumefaciens- } \\
\text { mediated transformation of plants } \\
\text { for deletion of } C R K 7 \text { via } \\
\text { CRISPR/ Cas } 9 .\end{array}$ & $\begin{array}{l}\text { Spec }^{\mathrm{R}}, \text { Basta }^{\circledR R}, \\
\text { seedGFP }\end{array}$ & This work \\
\hline $\begin{array}{l}\text { pB-crispr-seed GFP- } \\
E E-C R K 7^{B+F} \\
\text { (pCT7) }\end{array}$ & $\begin{array}{l}\text { Binary vector for } A \text {. tumefaciens- } \\
\text { mediated transformation of plants } \\
\text { for deletion of } C R K 7 \text { via } \\
\text { CRISPR/ Cas } 9 \text {. }\end{array}$ & $\begin{array}{l}\text { Spec }^{\mathrm{R}} \text {, Basta } \\
\text { seedGFP }\end{array}$ & This work \\
\hline $\begin{array}{l}\text { pB-crispr-seedGFP- } \\
E E-C R K 7^{C+D} \\
(\mathrm{pCT} 23)\end{array}$ & $\begin{array}{l}\text { Binary vector for } A \text {. tumefaciens- } \\
\text { mediated transformation of plants } \\
\text { for deletion of } C R K 7 \text { via } \\
\text { CRISPR/ Cas } 9 .\end{array}$ & $\begin{array}{l}\text { Spec }^{\mathrm{R}}, \text { Basta }^{\circledR R} \text {, } \\
\text { seedGFP }\end{array}$ & This work \\
\hline $\begin{array}{l}\text { pB-crispr-seed GFP- } \\
E E-C R K 7^{C+F} \\
\text { (pCT17) }\end{array}$ & $\begin{array}{l}\text { Binary vector for } A \text {. tumefaciens- } \\
\text { mediated transformation of plants } \\
\text { for deletion of } C R K 7 \text { via } \\
\text { CRISPR/ Cas } 9 \text {. }\end{array}$ & $\begin{array}{l}\text { Spec }^{\mathrm{R}}, \text { Basta }^{\mathbb{R}} \text {, } \\
\text { seedGFP }\end{array}$ & This work \\
\hline $\begin{array}{l}\text { pGreenII-0229- } \\
\text { pCRK43::crk43 } \\
\text { (pCT41) }\end{array}$ & $\begin{array}{l}\text { Binary vector for } A \text {. tumefaciens- } \\
\text { mediated transformation of plants } \\
\text { and expression of the } C R K 43 \\
\text { gDNA under control of } p C R K 43 \text {. }\end{array}$ & $\operatorname{Kan}^{\mathrm{R}}$, Basta ${ }^{\circledR R}$ & This work \\
\hline $\begin{array}{l}\text { pGreenII-0229- } \\
\text { pCRK43::crk43- } \\
\text { mCitrine } e^{\mathrm{d}} \\
\text { (pCT43) }\end{array}$ & $\begin{array}{l}\text { Binary vector for } A \text {. tumefaciens- } \\
\text { mediated transformation of plants } \\
\text { and expression of the } C R K 43 \\
\text { gDNA mit a C-terminal mCitrine- } \\
\text { tag under control of } p C R K 43 \text {. }\end{array}$ & $\operatorname{Kan}^{\mathrm{R}}$, Basta ${ }^{\circledR R}$ & This work \\
\hline
\end{tabular}

${ }^{a}$ Linker sequence: YAGAAA

${ }^{b}$ Linker sequence: GAGAGAAA

c Linker sequence: FE

d Linker sequence: YAGAAAGAGA

\subsubsection{Oligonucleotides}

The primers used in this study were ordered from Thermo Fisher Scientific (Waltham, Massachusetts, USA). The lyophilized oligonucleotides were diluted to a stock-concentration of $100 \mu \mathrm{M}$ with ultrapure water. For standard usage, aliquots with a working concentration of 10 $\mu \mathrm{M}$ were prepared by dilution with $\mathrm{ddH}_{2} \mathrm{O}$. Oligonucleotides were stored at $-20^{\circ} \mathrm{C}$. All oligonucleotides used in this study are listed in table 6. 
Table 6: Oligonucleotides used in this study.

Restriction enzyme cutting sites are underlined. guide RNA sequences are written in red.

\begin{tabular}{|c|c|c|}
\hline Primer & Sequence $\left(5^{\prime}-3^{\prime}\right)$ & Use \\
\hline \multicolumn{3}{|c|}{ Primers for genotyping } \\
\hline ЕР 64 & ATTTTGCCGATTTCGGAAC & SALK left border primer \\
\hline EP 23 & CTTCAACGTTGCGGTTCTGTCAGTT & SALK right border primer \\
\hline EP 294 & CCCATTTGGACGTGAATGTAGACAC & GABI left border primer \\
\hline EP 295 & GATCAGATTGTCGTTTCCCGCCTTCAG & GABI right border primer \\
\hline UL154 & ТСТTCTTCCCCACAGAGCAACGACG & \multirow{2}{*}{ Genotyping of cerk1-4 } \\
\hline UL166 & TTCCAGGCACATAAACGATTCC & \\
\hline oCT3 & $\begin{array}{l}\text { GGTGTTGTTGACGGCAAAGGTGACGCAGTGC- } \\
\text { CGG }\end{array}$ & \multirow{2}{*}{ Genotyping of $c r k 7-4$} \\
\hline oCT4 & CGCCTCTTACTCCACCGGATTCC & \\
\hline oCT26 & GATCCTCGTTTCCTAGC & \multirow{2}{*}{ Genotyping of $c r k 7-5$} \\
\hline oCT27 & AGTTGCAGCGACTCTATGG & \\
\hline oCT142 & $\begin{array}{l}\text { GCAATAGTTGCAATAACTGTTTGACTGATTGA- } \\
\text { TCCTA }\end{array}$ & \multirow{2}{*}{ Genotyping of $c r k 7-6$} \\
\hline oCT143 & GACCGATATGGGTGCATCGAAC & \\
\hline oCT25 & TGTACCAATGGCGGTTTCG & \multirow{3}{*}{$\begin{array}{l}\text { Genotyping of } c r k 7-7 \text { and } \\
\text { crk } 7-8\end{array}$} \\
\hline oCT131 & CAGTGGAAGGTTCGCCTACAAAG & \\
\hline oCT132 & CGGCGCCAAGCAGAATAATCATC & \\
\hline оCT91 & CCTTTTGAACGTCATCCTCAG & \multirow{2}{*}{ Genotyping of $c r k 43-1$} \\
\hline оСТ92 & ATCCCATGGGAAGGTAACAAC & \\
\hline оСТ90 & CTAGTGGCATTACTCCGAGTTG & \multirow{2}{*}{ Genotyping of $c r k 43-2$} \\
\hline оСТ96 & CCCGACCCATAAACTCGGTGAAG & \\
\hline оСТ93 & TTCATCCAGCAAAATATTGCC & \multirow{2}{*}{ Genotyping of $c r k 43-3$} \\
\hline оCT94 & TCTTCCCTGAATCAAAAACCC & \\
\hline \multicolumn{3}{|c|}{ Primers for expression analysis } \\
\hline Actin_fw & TGCGACAATGGAACTGGAATG & \multirow{2}{*}{$\begin{array}{l}\text { semi-quantitative RT-PCR of } \\
\text { ACTIN1 }\end{array}$} \\
\hline Actin_rev & GGATAGCATGTGGAAGTGCATAC & \\
\hline EP13 & AGACTCATACACTCTGGTGGGCCTT & \multirow{2}{*}{$\begin{array}{l}\text { semi-quantitative RT-PCR of } \\
\text { PRl }\end{array}$} \\
\hline EP14 & CGTCCTTTATGTACGTGTGTATGCA & \\
\hline EP223 & GGTTTTCCCCAGTGTTGTTG & \multirow{2}{*}{$\begin{array}{l}\text { quantitative RT-PCR of } \\
\text { ACTIN } 8\end{array}$} \\
\hline EP224 & CTCCATGTCATCCCAGTTGC & \\
\hline
\end{tabular}




\begin{tabular}{|c|c|c|}
\hline $\begin{array}{l}\text { EP227 } \\
\text { EP228 }\end{array}$ & $\begin{array}{l}\text { TGATCCTCGTGGGAATTATGT } \\
\text { TGCATGATCACATCATTACTTCAT }\end{array}$ & $\begin{array}{l}\text { quantitative RT-PCR of } \\
\text { PRI }\end{array}$ \\
\hline JE 30 & GAAGAGTTTGCCGATGGAGG & \multirow{2}{*}{$\begin{array}{l}\text { semi-quantitative RT-PCR } \\
\text { of } W R K Y 53\end{array}$} \\
\hline JE 31 & CGAGGCTAATGGTGGTGTTC & \\
\hline JE 78 & GCAGCTTGAGAGCAAGAATG & \multirow{2}{*}{$\begin{array}{l}\text { semi-quantitative RT-PCR } \\
\text { of } W R K Y 30\end{array}$} \\
\hline EP108 & TCAAGAACCACTTCTCATCAAGA & \\
\hline oCT26 & GATCCTCGTTTCCTAGC & \multirow{2}{*}{$\begin{array}{l}\text { semi-quantitative RT-PCR } \\
\text { of } C R K 7\end{array}$} \\
\hline oCT27 & AGTTGCAGCGACTCTATGG & \\
\hline oCT36 & CAAGCCGGTTTGTTCC & \multirow{2}{*}{$\begin{array}{l}\text { semi-quantitative RT-PCR } \\
\text { of } C R K 7\end{array}$} \\
\hline oCT49 & ACTGGACACAGCGATAC & \\
\hline oCT76 & ATGTCTTCTCTCTTCССTTTC & \multirow{2}{*}{$\begin{array}{l}\text { semi-quantitative RT-PCR } \\
\text { of } C R K 7 \text { (intron spanning) }\end{array}$} \\
\hline oCT78 & TTATCATCTTCATCTAAAGCAGG & \\
\hline oCT79 & CCTGCTTTAGATGAAGATGATAA & \multirow{2}{*}{$\begin{array}{l}\text { semi-quantitative RT-PCR } \\
\text { of } C R K 7 \text { (intron spanning) }\end{array}$} \\
\hline oCT36 & CAAGCCGGTTTGTTCC & \\
\hline оСТ90 & CTAGTGGCATTACTCCGAGTTG & \multirow{2}{*}{$\begin{array}{l}\text { semi-quantitative RT-PCR } \\
\text { of } C R K 43\end{array}$} \\
\hline оСТ95 & CGCCAAACTGCATCATCCACAGG & \\
\hline oCT89 & CCTTGTCGAGGCTCTCATTAAC & \multirow{2}{*}{$\begin{array}{l}\text { semi-quantitative RT-PCR } \\
\text { of } C R K 43\end{array}$} \\
\hline oCT124 & ATGGTCACTAAGAACTCTCATAAG & \\
\hline oCT87 & CTGCAACACGAGTGTTGAC & \multirow{2}{*}{$\begin{array}{l}\text { semi-quantitative RT-PCR } \\
\text { of } C R K 43\end{array}$} \\
\hline оСТ96 & CCCGACCCATAAACTCGGTGAAG & \\
\hline \multicolumn{3}{|c|}{ Primers for cloning } \\
\hline oCT7 & ACTGCAGGCGCGCCCCAGGTTTTGACGTCTATC & \multirow{2}{*}{$\begin{array}{l}\text { Cloning } p C R K 7:: C R K 7 \text { and } \\
p C R K 7:: c r k 7-4\end{array}$} \\
\hline оCT8 & $\begin{array}{l}\text { GCTGGCAGATCTTCAACGAGGATCTAAATCAGA- } \\
\text { C }\end{array}$ & \\
\hline oCT43 & $\begin{array}{l}\text { GACCTGCAGGTCCGGGATCCATGTCTTCTCTCTT- } \\
\text { CCC }\end{array}$ & \multirow{2}{*}{$\begin{array}{l}\text { Cloning } p 35 S:: C R K 7 \text { and } \\
\text { p35S::crk7-4 }\end{array}$} \\
\hline oCT56 & CCCCCGGGTCAACGAGGATCTAAATCAGAC & \\
\hline oCT7 & ACTGCAGGCGCGCCCCAGGTTTTGACGTCTATC & \multirow{2}{*}{$\begin{array}{l}\text { Cloning } p C R K 7:: C R K 7- \\
\text { Citrine and } p C R K 7:: \text { crk7-4- } \\
\text { Citrine }\end{array}$} \\
\hline оCT9 & $\begin{array}{l}\text { GATGTGGCGGCCGCTCCAGCGTAACGAGGATCT- } \\
\text { AAATCAGACATTG }\end{array}$ & \\
\hline EP533 & GGTACCGGCGCGCCGGTCCGGTCCCCAG & \multirow{2}{*}{$\begin{array}{l}\text { Cloning } p 35 S:: C R K 7- \\
\text { Citrine and } p 35 S:: c r k 7-4- \\
\text { Citrine }\end{array}$} \\
\hline оCT68 & $\begin{array}{l}\text { CCATGGCGGCCGCTCCCGCTCCCGCTCCACGAG- } \\
\text { GATCTAAATCAGACATTG }\end{array}$ & \\
\hline
\end{tabular}




\begin{tabular}{|c|c|c|}
\hline оCT97 & ATTTGTTTCATTGATTC $\underline{\text { GTTAACCAGTCCTG }}$ & \multirow{4}{*}{ Cloning $p 35 S:: C R K 7-F L A G$} \\
\hline оСТ99 & $\begin{array}{l}\text { TGGTCTTTGTAGTCTTCGAAAACGAGGATCTAA- } \\
\text { ATCAGACATTG }\end{array}$ & \\
\hline oCT100 & $\begin{array}{l}\text { CAATGTCTGATTTAGATCCTCGTTTCGAAGAC- } \\
\text { TACAAAGACCATG }\end{array}$ & \\
\hline oCT101 & $\begin{array}{l}\text { AGAACTAGTGGATCCCCCGGGCTATCAC- } \\
\text { TTATCGTCATCGTCCTTG }\end{array}$ & \\
\hline oCT104 & $\begin{array}{l}\text { ATATATGGTCTCTGATTGATTAACGTGAAATAT- } \\
\text { ACGGGTTTTAGAGCTAGAAATAGCAAG }\end{array}$ & \multirow{2}{*}{$\begin{array}{l}\text { Cloning } p B \text {-crispr- } \\
\text { seedGFP-EE-CRK } 7^{B+F}\end{array}$} \\
\hline oCT105 & $\begin{array}{l}\text { ATTATTGGTCTCTAAACCTTTACAAATCATGCAA- } \\
\text { ATCAATCTCTTAGTCGACTCTACC }\end{array}$ & \\
\hline oCT104 & $\begin{array}{l}\text { ATATATGGTCTCTGATTGATTAACGTGAAATAT- } \\
\text { ACGGGTTTTAGAGCTAGAAATAGCAAG }\end{array}$ & \multirow{2}{*}{$\begin{array}{l}\text { Cloning } p B \text {-crispr- } \\
\text { seed } G F P-E E-C R K 7^{B+E}\end{array}$} \\
\hline oCT106 & $\begin{array}{l}\text { ATTATTGGTCTCTAAACACTGTACTCTGTTTTCC- } \\
\text { CTCAATCTCTTAGTCGACTCTACC }\end{array}$ & \\
\hline oCT104 & $\begin{array}{l}\text { ATATATGGTCTCTGATTGATTAACGTGAAATAT- } \\
\text { ACGGGTTTTAGAGCTAGAAATAGCAAG }\end{array}$ & \multirow{2}{*}{$\begin{array}{l}\text { Cloning } p B \text {-crispr- } \\
\text { seed } G F P-E E-C R K 7^{B+D}\end{array}$} \\
\hline oCT107 & $\begin{array}{l}\text { ATTATTGGTCTCTAAACAAATGAGAGGGAGAGA- } \\
\text { ACACAATCTCTTAGTCGACTCTACC }\end{array}$ & \\
\hline oCT105 & $\begin{array}{l}\text { ATTATTGGTCTCTAAACCTTTACAAATCATGCAA- } \\
\text { ATCAATCTCTTAGTCGACTCTACC }\end{array}$ & \multirow{2}{*}{$\begin{array}{l}\text { Cloning } p B \text {-crispr- } \\
\text { seedGFP-EE-CRK } 7^{A+F}\end{array}$} \\
\hline oCT108 & $\begin{array}{l}\text { ATATATGGTCTCTGATTGCTCCCATATTATTTA- } \\
\text { AGAGGGTTTTAGAGCTAGAAATAGCAAG }\end{array}$ & \\
\hline оCT106 & $\begin{array}{l}\text { ATTATTGGTCTCTAAACACTGTACTCTGTTTTCC- } \\
\text { CTCAATCTCTTAGTCGACTCTACC }\end{array}$ & \multirow{2}{*}{$\begin{array}{l}\text { Cloning } p B \text {-crispr- } \\
\text { seedGFP-EE-CRK } 7^{A+E}\end{array}$} \\
\hline oCT108 & $\begin{array}{l}\text { ATATATGGTCTCTGATTGCTCCCATATTATTTA- } \\
\text { AGAGGGTTTTAGAGCTAGAAATAGCAAG }\end{array}$ & \\
\hline oCT107 & $\begin{array}{l}\text { ATTATTGGTCTCTAAACAAATGAGAGGGAGAGA- } \\
\text { ACACAATCTCTTAGTCGACTCTACC }\end{array}$ & \multirow{2}{*}{$\begin{array}{l}\text { Cloning } p B \text {-crispr- } \\
\text { seedGFP-EE-CRK } 7^{A+D}\end{array}$} \\
\hline oCT108 & $\begin{array}{l}\text { ATATATGGTCTCTGATTGCTCCCATATTATTTA- } \\
\text { AGAGGGTTTTAGAGCTAGAAATAGCAAG }\end{array}$ & \\
\hline oCT105 & $\begin{array}{l}\text { ATTATTGGTCTCTAAACCTTTACAAATCATGCAA- } \\
\text { ATCAATCTCTTAGTCGACTCTACC }\end{array}$ & \multirow{2}{*}{$\begin{array}{l}\text { Cloning } p B \text {-crispr- } \\
\text { seedGFP-EE-CRK } 7^{C+F}\end{array}$} \\
\hline oCT109 & $\begin{array}{l}\text { ATATATGGTCTCTGATTGATAATTTGTCAGTTTA- } \\
\text { TAAAGTTTTAGAGCTAGAAATAGCAAG }\end{array}$ & \\
\hline oCT107 & $\begin{array}{l}\text { ATTATTGGTCTCTAAACAAATGAGAGGGAGAGA- } \\
\text { ACACAATCTCTTAGTCGACTCTACC }\end{array}$ & \multirow{2}{*}{$\begin{array}{l}\text { Cloning } p B-c r i s p r- \\
\text { seed } G F P-E E-C R K 7^{C+D}\end{array}$} \\
\hline oCT109 & $\begin{array}{l}\text { ATATATGGTCTCTGATTGATAATTTGTCAGTTTA- } \\
\text { TAAAGTTTTAGAGCTAGAAATAGCAAG }\end{array}$ & \\
\hline oCT122 & $\begin{array}{l}\text { TAGGGCGAATTGGGTACCGGCGCGCCAGAAAA- } \\
\text { CACTACACTAAATG }\end{array}$ & \multirow{2}{*}{ Cloning $p C R K 43:: C R K 43$} \\
\hline oCT125 & $\begin{array}{l}\text { GACTCTAGAACTAGTGGATCCTCAATAACTCAT- } \\
\text { ATGTCGTTTCC }\end{array}$ & \\
\hline EP594 & CTACCAGTCCAAGCTGAGCAAAGACC & \multirow{2}{*}{$\begin{array}{l}\text { Cloning } p C R K 43:: C R K 43- \\
\text { mCitrine }\end{array}$} \\
\hline EP595 & CTCAGGTAGTGGTTGTCG & \\
\hline
\end{tabular}




\begin{tabular}{|c|c|c|}
\hline \multicolumn{3}{|c|}{ Primers for sequencing } \\
\hline EP23 & CTTCAACGTTGCGGTTCTGTCAGTT & \multirow{6}{*}{$\begin{array}{l}\text { Sequencing of } \\
p C R K 7:: C R K 7 \text { and } \\
p C R K 7:: c r k 7-4\end{array}$} \\
\hline EP156 & AGCTTGCCGTAGGTGGCATC & \\
\hline EP218 & CTATAAGAACCCTAATTCCCTTATCTG & \\
\hline oCT3 & $\begin{array}{l}\text { GGTGTTGTTGACGGCAAAGGTGACGCAG- } \\
\text { TGCCGG }\end{array}$ & \\
\hline oCT4 & CGCCTCTTACTCCACCGGATTCC & \\
\hline oCT14 & GCAAAGAGGGCAAAGAAG & \\
\hline EP23 & CTTCAACGTTGCGGTTCTGTCAGTT & \multirow{11}{*}{$\begin{array}{l}\text { Sequencing of } p 35 S:: C R K 7 \\
\text { and } p 35 S: \because c r k 7-4\end{array}$} \\
\hline EP136 & CGCTCATGTGTTGAGCATATAAG & \\
\hline EP166 & CCAACAGTTGCGCAGCCTGAATG & \\
\hline EP167 & GGATTAGCAGAGCGAGGTATGTAG & \\
\hline EP168 & AGTAACACCACACCGCTCATTGTC & \\
\hline EP270 & GTAATACGACTCACTATAGGGCGAATTG & \\
\hline CM19 & TTGAATCCTGTTGCCGGTCTTG & \\
\hline $\mathrm{CM} 22$ & CTCCGATTAGCGATTTCACGTACC & \\
\hline YZ96 & GTGGATTGATGTGATATCTCC & \\
\hline oCT20 & CCAATATGGAACGCAGAG & \\
\hline оCT49 & ACTGGACACAGCGATAC & \\
\hline EP23 & CTTCAACGTTGCGGTTCTGTCAGTT & \multirow{7}{*}{$\begin{array}{l}\text { Sequencing of } \\
p C R K 7:: C R K 7-C i t r i n e \text { and } \\
p C R K 7:: c r k 7-4-C i t r i n e\end{array}$} \\
\hline EP156 & AGCTTGCCGTAGGTGGCATC & \\
\hline EP172 & TTCTTCTGCTTGTCGGCCATGATA & \\
\hline oCT3 & $\begin{array}{l}\text { GGTGTTGTTGACGGCAAAGGTGACGCAG- } \\
\text { TGCCGG }\end{array}$ & \\
\hline oCT4 & CGCCTCTTACTCCACCGGATTCC & \\
\hline oCT9 & $\begin{array}{l}\text { GATGTGGCGGCCGCTCCAGCGTAACGAG- } \\
\text { GATCTAAATCAGACATTG }\end{array}$ & \\
\hline oCT14 & GCAAAGAGGGCAAAGAAG & \\
\hline EP23 & CTTCAACGTTGCGGTTCTGTCAGTT & \multirow{4}{*}{$\begin{array}{l}\text { Sequencing of } p 35 S:: C R K 7- \\
\text { Citrine and } p 35 S:: c r k 7-4- \\
\text { Citrine }\end{array}$} \\
\hline YZ96 & GTGGATTGATGTGATATCTCC & \\
\hline оCT20 & CCAATATGGAACGCAGAG & \\
\hline оCT49 & ACTGGACACAGCGATAC & \\
\hline
\end{tabular}




\begin{tabular}{|c|c|c|}
\hline YZ96 & GTGGATTGATGTGATATCTCC & \multirow{5}{*}{$\begin{array}{l}\text { Sequencing of } p 35 S:: C R K 7- \\
F L A G\end{array}$} \\
\hline oCT20 & CCAATATGGAACGCAGAG & \\
\hline oCT53 & TGCCGGAAGAGTGAAGTG & \\
\hline оCT73 & TGTTGGTCGTTGATTGATCTG & \\
\hline оСТ97 & ATTTGTTTCATTGATTCGTTAACCAGTCCTG & \\
\hline EP23 & CTTCAACGTTGCGGTTCTGTCAGTT & \multirow{12}{*}{$\begin{array}{l}\text { Sequencing of } \\
\text { pCRK7::CRK7-mCitrine, } \\
\text { pCRK7::crk7-4-mCitrine, } \\
\text { p35S::CRK7-mCitrine and } \\
\text { p35S::crk7-4-mCitrine }\end{array}$} \\
\hline EP136 & CGCTCATGTGTTGAGCATATAAG & \\
\hline EP166 & CCAACAGTTGCGCAGCCTGAATG & \\
\hline EP167 & GGATTAGCAGAGCGAGGTATGTAG & \\
\hline EP168 & AGTAACACCACACCGCTCATTGTC & \\
\hline EP204 & GAGCTCTCACTTCCTGAGAATCTC & \\
\hline $\mathrm{CM} 22$ & CTCCGATTAGCGATTTCACGTACC & \\
\hline oCT4 & CGCCTCTTACTCCACCGGATTCC & \\
\hline oCT14 & GCAAAGAGGGCAAAGAAG & \\
\hline oCT24 & AGAGGTGGAGAAACAATG & \\
\hline oCT49 & ACTGGACACAGCGATAC & \\
\hline oCT53 & TGCCGGAAGAGTGAAGTG & \\
\hline EP23 & CTTCAACGTTGCGGTTCTGTCAGTT & \multirow{7}{*}{$\begin{array}{l}\text { Sequencing of } \\
p C R K 43:: C R K 43\end{array}$} \\
\hline EP218 & CTATAAGAACCCTAATTCCCTTATCTG & \\
\hline оСТ89 & CCTTGTCGAGGCTCTCATTAAC & \\
\hline oCT94 & TCTTCCСТGAATCAAAAACCC & \\
\hline оCT95 & CGCCAAACTGCATCATCCACAGG & \\
\hline оСТ96 & CCCGACCCATAAACTCGGTGAAG & \\
\hline oCT123 & $\begin{array}{l}\text { TTATGAGAGTTCTTAGTGACCATCCCATGG- } \\
\text { GAAGGTAACAAC }\end{array}$ & \\
\hline EP23 & CTTCAACGTTGCGGTTCTGTCAGTT & \multirow{8}{*}{$\begin{array}{l}\text { Sequencing of } \\
p C R K 43:: C R K 43-m \text { Citrine }\end{array}$} \\
\hline EP166 & CCAACAGTTGCGCAGCCTGAATG & \\
\hline EP169 & TTGAATCCTGTTGCCGGTCTTG & \\
\hline EP402 & TCAAGGAGGACGGCAACATCCTG & \\
\hline EP545 & ATTTCGGTGACGGGCAGGAC & \\
\hline JE23 & ATGGTGAGCAAGGGCGAGGAGC & \\
\hline oHG40 & TCGTGCTGCTTCATGTGGTC & \\
\hline oHG144 & GAGGCACAGGGCTTCAAGAG & \\
\hline
\end{tabular}




\begin{tabular}{|c|c|c|}
\hline оСТ92 & ATCCCATGGGAAGGTAACAAC & \multirow{5}{*}{$\begin{array}{l}\text { Sequencing of } \\
p C R K 43:: C R K 43-m \text { Citrine }\end{array}$} \\
\hline оСТ94 & TCTTCCCTGAATCAAAAACCC & \\
\hline оCT95 & CGCCAAACTGCATCATCCACAGG & \\
\hline оСТ96 & CCCGACCCATAAACTCGGTGAAG & \\
\hline oCT116 & $\begin{array}{l}\text { GCTGGAGCGGCCGCCGGCGCAGGGG- } \\
\text { CAATGGTGAGCAAGGGCGAGGAG }\end{array}$ & \\
\hline oCT117 & CAAAAGGCCCCTGGGAATC & \multirow{2}{*}{$\begin{array}{l}\text { Sequencing of } p B \text {-crispr- } \\
\text { seed } G F P-E E-C R K 7\end{array}$} \\
\hline oCT118 & CACCGGTCTAGACGCGTCTC & \\
\hline EP64 & ATTTTGCCGATTTCGGAAC & $\begin{array}{l}\text { Sequencing of } c r k 43-1 \text { and } \\
\text { crk43-3 PCR products }\end{array}$ \\
\hline EP294 & CCCATTTGGACGTGAATGTAGACAC & \multirow{2}{*}{$\begin{array}{l}\text { Sequencing of } c r k 7-5 \text { PCR } \\
\text { products }\end{array}$} \\
\hline oCT27 & AGTTGCAGCGACTCTATGG & \\
\hline oCT29 & ATCACCATCCCACTGTACTC & \multirow{2}{*}{$\begin{array}{l}\text { Sequencing of } c r k 7-6 \text { PCR } \\
\text { products }\end{array}$} \\
\hline oCT46 & TTCTGCTTGGCGCCGAAAC & \\
\hline оСТ96 & CCCGACCCATAAACTCGGTGAAG & $\begin{array}{l}\text { Sequencing of } c r k 43-2 \text { RT- } \\
\text { PCR products }\end{array}$ \\
\hline oCT131 & CAGTGGAAGGTTCGCCTACAAAG & \multirow{2}{*}{$\begin{array}{l}\text { Sequencing of } c r k 7-7 \text { and } \\
\text { crk7-8 PCR products }\end{array}$} \\
\hline oCT132 & CGGCGCCAAGCAGAATAATCATC & \\
\hline
\end{tabular}

\section{$\underline{2.1 .6 \text { Enzymes }}$}

2.1.6.1 Restriction endonucleases

Restriction endonucleases were obtained from Thermo Fisher Scientific (Waltham, USA). They were used with the supplied 10x reaction buffers according to the manufacturer's recommendations.

\subsubsection{Polymerases and nucleic acid modifying enzymes}

Homemade Taq DNA polymerase was used for standard polymerase chain reactions (PCR, 2.2.3.4.1). PCR products for cloning were amplified with the proofreading iProof ${ }^{\mathrm{TM}}$ HighFidelity DNA polymerase according to the manufacturer's instructions (Bio-Rad, Munich, Germany, 2.2.3.4.2). cDNA was synthesized from total RNA using the RevertAid ${ }^{\mathrm{TM}} \mathrm{H}$ Minus Reverse Transcriptase (Thermo Fisher Scientific, Waltham, USA) according to the manufacturer's instructions (2.2.3.14).

The T4 DNA ligase (Thermo Fisher Scientific, Waltham, USA) was used for ligations. 


\subsubsection{Trypsin}

Sequencing Grade Modified Trypsin (Promega, Madison, Wisconsin, USA) was used for tryptic digestion of proteins prior to MS analysis.

\subsubsection{Chemicals}

All chemicals used in this work were purchased from one of the following manufacturers: BioRad (Munich, Germany), Duchefa (Haarlem, The Netherlands), Intas (Göttingen, Germany), Invitrogen (Karlsruhe, Germany), Promega (Madison, Wisconsin, USA), Roth (Karlsruhe, Germany), Sigma-Aldrich (Munich, Germany), Thermo Fisher Scientific (Waltham, USA) or VWR (Darmstadt, Germany).

\section{$\underline{2.1 .8 \text { Antibodies }}$}

Primary and secondary antibodies used for immunoblotting in this work are listed in table 7. The primary antibody $\alpha$-pMAPK Phospho p $44 / 42$ was stored at $-20^{\circ} \mathrm{C}$, whereas all other antibodies were aliquoted and stored at $-80^{\circ} \mathrm{C}$ for long term storage. Aliquots of antibodies in use were kept at $4^{\circ} \mathrm{C}$ or $-20^{\circ} \mathrm{C}$.

Table 7: Primary and secondary antibodies used in this study.

\begin{tabular}{|c|c|c|}
\hline Primary antibody & Source organism & Company \\
\hline $\begin{array}{l}\alpha-C E R K 1 \\
\text { used: } 1: 3000\end{array}$ & $\begin{array}{l}\text { Rabbit, } \\
\text { polyclonal }\end{array}$ & Eurogentec (Cologne, Germany) \\
\hline $\begin{array}{l}\alpha \text {-DYKDDDDK (binds to } \\
\text { Sigma FLAG }{ }^{\circledR} \text { ), } \\
\text { used: } 1: 3000\end{array}$ & $\begin{array}{l}\text { Mouse, } \\
\text { monoclonal }\end{array}$ & Agrisera (Vännäs, Sweden) \\
\hline $\begin{array}{l}\alpha \text {-GFP, } \\
\text { used: } 1: 3000\end{array}$ & $\begin{array}{l}\text { Rat, } \\
\text { monoclonal }\end{array}$ & $\begin{array}{l}\text { ChromoTek GmbH (Planegg- } \\
\text { Martinsried, Germany) }\end{array}$ \\
\hline $\begin{array}{l}\alpha \text {-pMAPK Phospho p44/42 } \\
\text { (Erk1/2) (Thr202/Tyr204), } \\
\text { used: 1:5000 }\end{array}$ & $\begin{array}{l}\text { Rabbit, } \\
\text { polyclonal }\end{array}$ & $\begin{array}{l}\text { Cell Signaling Technology (Dan- } \\
\text { vers, MA, USA) }\end{array}$ \\
\hline
\end{tabular}




\begin{tabular}{lll}
\hline Secondary antibody & Source organism & Company \\
\hline $\begin{array}{l}\alpha \text {-rat IgG AP conjugate } \\
\text { used 1:5000 }\end{array}$ & $\begin{array}{l}\text { Rabbit, } \\
\text { polyclonal }\end{array}$ & Sigma-Aldrich (Munich, Germany) \\
$\begin{array}{l}\alpha \text {-rabbit IgG AP conjugate } \\
\text { used: 1:5000 }\end{array}$ & $\begin{array}{l}\text { Goat, } \\
\text { polyclonal }\end{array}$ & Sigma-Aldrich (Munich, Germany) \\
$\begin{array}{l}\alpha-\text {-mouse IgG AP conjugate } \\
\text { used 1:5000 }\end{array}$ & $\begin{array}{l}\text { Goat, } \\
\text { polyclonal }\end{array}$ & Sigma-Aldrich (Munich, Germany) \\
\hline
\end{tabular}

\subsubsection{Antibiotics}

The antibiotics used in this work are summarized in table 8. Antibiotic stock solutions were stored at $-20^{\circ} \mathrm{C}$ and used in $1: 1000$ dilutions.

Table 8: Antibiotics used in this study.

\begin{tabular}{llll}
\hline Antibiotic & Stock concentration & Final concentration & Solvent \\
\hline Carbenicillin (Carb) & $50 \mathrm{mg} / \mathrm{ml}$ & $50 \mu \mathrm{g} / \mathrm{ml}$ & $\mathrm{ddH}_{2} \mathrm{O}$ \\
Gentamycin (Gent) & $50 \mathrm{mg} / \mathrm{ml}$ & $50 \mu \mathrm{g} / \mathrm{ml}$ & $\mathrm{ddH}_{2} \mathrm{O}$ \\
Kanamycin (Kan) & $50 \mathrm{mg} / \mathrm{ml}$ & $50 \mu \mathrm{g} / \mathrm{ml}$ & $\mathrm{ddH}_{2} \mathrm{O}$ \\
Rifampicin (Rif) & $20 \mathrm{mg} / \mathrm{ml}$ & $20 \mu \mathrm{g} / \mathrm{ml}$ & $\mathrm{DMSO}$ \\
Spectinomycin (Spec) & $100 \mathrm{mg} / \mathrm{ml}$ & $100 \mu \mathrm{g} / \mathrm{ml}$ & $\mathrm{ddH}_{2} \mathrm{O}$ \\
Tetracyclin (Tet) & $5 \mathrm{mg} / \mathrm{ml}$ & $5 \mu \mathrm{g} / \mathrm{ml}$ & Ethanol \\
\hline
\end{tabular}

\section{$\underline{2.1 .10 \text { Media }}$}

Media were prepared using ultrapure water and autoclaved after preparation at $121^{\circ} \mathrm{C}$ for 20 min. Antibiotics were added after cooling down to $60^{\circ} \mathrm{C}$ or lower. Liquid and solid media without antibiotics were stored at room temperature; liquid and solid media with antibiotics were stored at $4^{\circ} \mathrm{C}$. Media used in this study are listed in table 9 . 
Table 9: Growth media used in this study.

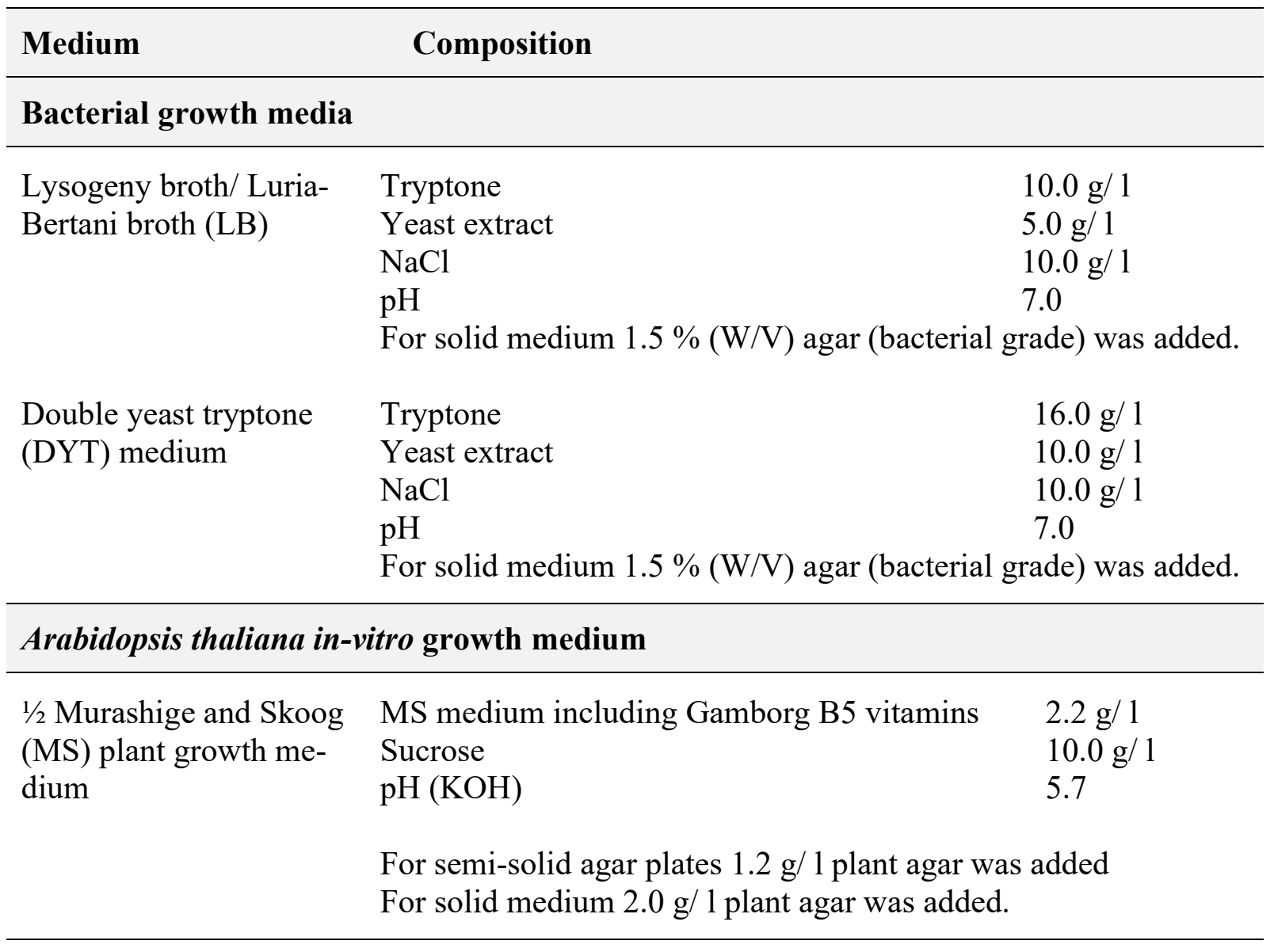

\subsubsection{Buffers and solutions}

All buffers and solutions were prepared with $\mathrm{ddH}_{2} \mathrm{O}$ and sterilized by autoclaving for 20 min at $121^{\circ} \mathrm{C}$. Buffers and solutions which were not autoclaved were sterilized using filters with a pore size of $0.2 \mu \mathrm{m}$. Table 10 lists the buffers and solutions used in this work.

Table 10: Buffers and Solutions used in this study.

\begin{tabular}{lll}
\hline Buffer/ Solution & \multicolumn{1}{c}{ Composition } \\
\hline Agarose gel electrophoresis and PCR & \\
\hline Agarose solution & Agarose & $1-3 \%(\mathrm{w} / \mathrm{v})$ \\
& TAE-Buffer & $1 \mathrm{x}$ \\
DNA loading dye (6x) & Sucrose & $4 \mathrm{~g}$ \\
& $\begin{array}{l}0.5 \mathrm{M} \text { EDTA pH } 8.0 \\
\text { Bromophenol blue } \\
\text { dd }{ }_{2} \mathrm{O}\end{array}$ & $\begin{array}{l}2 \mathrm{ml} \\
\mathrm{mg}\end{array}$ \\
& & Add. $10 \mathrm{ml}$ \\
\hline
\end{tabular}




\begin{tabular}{|c|c|c|}
\hline PCR reaction buffer for Taq $(10 x)$ & $\begin{array}{l}\text { Tris base } \\
\mathrm{KCl} \\
\mathrm{MgCl}_{2} \\
\text { Triton X-100 } \\
\mathrm{pH}(\mathrm{KOH})\end{array}$ & $\begin{array}{l}100 \mathrm{mM} \\
500 \mathrm{mM} \\
15 \mathrm{mM} \\
1 \%(\mathrm{w} / \mathrm{v}) \\
9.0\end{array}$ \\
\hline TAE $(50 x)$ & $\begin{array}{l}\text { Tris base } \\
\text { Glacial acetic acid } \\
0.5 \text { M EDTA pH } 8.0\end{array}$ & $\begin{array}{l}2 \mathrm{M} \\
57.1 \mathrm{ml} / 1 \\
100 \mathrm{ml} / 1\end{array}$ \\
\hline \multicolumn{3}{|c|}{ Agrobacterium tumefaciens infiltration } \\
\hline Infiltration medium & $\begin{array}{l}\mathrm{MgCl}_{2} \\
\mathrm{MES} \\
\mathrm{pH} \\
\text { Add } 150 \mu \mathrm{M} \text { acetosyringone fro } \\
\text { solution prior to use. }\end{array}$ & $\begin{array}{l}10 \mathrm{mM} \\
10 \mathrm{mM} \\
5.4 \\
50 \mathrm{mM} \text { stock }\end{array}$ \\
\hline \multicolumn{3}{|c|}{ Genomic DNA extraction from plants } \\
\hline Extraction buffer & $\begin{array}{l}\text { Tris-HCl pH } 7.5 \\
\mathrm{NaCl} \\
\text { EDTA } \\
\text { SDS }\end{array}$ & $\begin{array}{l}0.2 \mathrm{M} \\
1.25 \mathrm{M} \\
0.025 \mathrm{M} \\
0.5 \%(\mathrm{w} / \mathrm{v})\end{array}$ \\
\hline \multicolumn{3}{|l|}{ In vitro phosphorylation assay } \\
\hline 10x kinase buffer & $\begin{array}{l}\text { HEPES-KOH pH } 7.5 \\
\mathrm{MgCl}_{2} \\
\mathrm{MnCl}_{2} \\
\text { Add } 1 \mathrm{mM} \text { DTT from a } 1 \mathrm{M} \text { sto } \\
\text { use. }\end{array}$ & $\begin{array}{l}20 \mathrm{mM} \\
10 \mathrm{mM} \\
5 \mathrm{mM} \\
\text { ution prior to }\end{array}$ \\
\hline Coomassie staining solution & $\begin{array}{l}\text { Methanol } \\
\text { Acetic acid } \\
\text { Coomassie Brilliant Blue R-250 }\end{array}$ & $\begin{array}{l}45 \% \\
10 \% \\
0.25 \%(\mathrm{w} / \mathrm{v})\end{array}$ \\
\hline Destaining solution & $\begin{array}{l}\text { Methanol } \\
\text { Acetic acid }\end{array}$ & $\begin{array}{l}25 \% \\
7 \%\end{array}$ \\
\hline \multicolumn{3}{|l|}{ Plasmid preparation ("mini-prep") } \\
\hline Buffer P1 & $\begin{array}{l}\text { Tris-HCl pH } 8.0 \\
\text { EDTA pH } 8.0 \\
\text { RNase A }\end{array}$ & $\begin{array}{l}50 \mathrm{mM} \\
10 \mathrm{mM} \\
100 \mu \mathrm{g} / \mathrm{ml}\end{array}$ \\
\hline Buffer P2 & $\begin{array}{l}\mathrm{NaOH} \\
\mathrm{SDS}\end{array}$ & $\begin{array}{l}200 \mathrm{mM} \\
1 \%(\mathrm{w} / \mathrm{v})\end{array}$ \\
\hline Buffer P3 & $\begin{array}{l}\text { Potassium acetate } \\
\text { Acetic acid }\end{array}$ & $\begin{array}{l}3 \mathrm{M} \\
2 \mathrm{M}\end{array}$ \\
\hline
\end{tabular}




\section{Preparation of chemically competent $E$. coli cells}

RF I solution

\section{$\mathrm{RbCl}$}

$100 \mathrm{mM}$

$\mathrm{MnCl}_{2}$

$50 \mathrm{mM}$

Potassium acetate

$30 \mathrm{mM}$

$\mathrm{CaCl}_{2} \times 2 \mathrm{H}_{2} \mathrm{O}$

$10 \mathrm{mM}$

Glycerol

pH (acetic acid)

$15 \%(\mathrm{w} / \mathrm{v})$

5.8

RF II solution

\section{MOPS}

$10 \mathrm{mM}$

$\mathrm{RbCl}$

$10 \mathrm{mM}$

$\mathrm{CaCl}_{2} \times 2 \mathrm{H}_{2} \mathrm{O}$

$75 \mathrm{mM}$

Glycerol

$15 \%(\mathrm{w} / \mathrm{v})$

$\mathrm{pH}(\mathrm{NaOH})$

6.8

\section{Protein extraction from plants}

CERK1 extraction buffer

Protease inhibitor cocktail (PIC)

$\begin{array}{ll}\text { Sucrose } & 250 \mathrm{mM} \\ \text { HEPES-KOH pH } 7.5 & 100 \mathrm{mM} \\ \text { Glycerol }_{\mathrm{Na}_{2} \mathrm{MoO}_{4}} & 5 \%(\mathrm{v} / \mathrm{v}) \\ \mathrm{NaF} & 1 \mathrm{mM} \\ \text { EDTA } & 25 \mathrm{mM} \\ \text { DTT } & 10 \mathrm{mM} \\ \text { Triton X-100 } & 1 \mathrm{mM} \\ \text { Protease inhibitor cocktail (PIC) } & 0.5 \%(\mathrm{w} / \mathrm{v}) \\ & 1: 100\end{array}$

4-(2-aminoethyl) benzenesulfonyl $1 \mathrm{~g}$ fluoride hydrochloride (AEBSF)

Bestatin hypochloride

Pepstatin A

$5 \mathrm{mg}$

Leupeptin hemisulfate

$10 \mathrm{mg}$

$100 \mathrm{mg}$

E-64 (trans-epoxysuccinyl-L-

$10 \mathrm{mg}$

leucylamido-(4-guanidino)butane)

Phenantroline (1, 10-phenantroline $10 \mathrm{~g}$ monohydrate)

All components were dissolved separately in a small amount of DMSO and then combined and filled up to $200 \mathrm{ml}$ with DMSO. The mixture was aliquoted in 2 $\mathrm{ml}$ and stored at $-20^{\circ} \mathrm{C}$. 


\section{Proteomics}

Elution buffer I

Tris-HCl pH 7.5

$50 \mathrm{mM}$

Urea

$2 \mathrm{M}$

Sequencing Grade Modified Trypsin $5 \mu \mathrm{g} / \mathrm{ml}$

DTT

$1 \mathrm{mM}$

Buffer has to be prepared freshly because of instability of urea solution.

Elution buffer II

Tris- $\mathrm{HCl} \mathrm{pH} 7.5$

$50 \mathrm{mM}$

Urea

Iodoacetamide (IAM)

$5 \mathrm{mM}$

Buffer has to be prepared freshly because of instability of urea solution.

Wash/ Dilution buffer

Tris-HCl pH 7.5

$10 \mathrm{mM}$

$\mathrm{NaCl}$

$150 \mathrm{mM}$

EDTA

$0.5 \mathrm{mM}$

ROS Burst analysis

Luminol solution

Luminol L-012

$100 \mu \mathrm{M}$

Horse Radish Peroxidase (HRP)

$20 \mu \mathrm{g} / \mu 1$

$\mathrm{ddH}_{2} \mathrm{O}$

Add $12 \mathrm{ml}$

Prepared stock solutions of L-012 $(20 \mathrm{mM})$ and HRP

$(10 \mathrm{mg} / \mathrm{ml})$ were used and can be stored $-20^{\circ} \mathrm{C}$.

SDS-PAGE and immunoblot analysis

\begin{tabular}{lll}
\hline 4 4x SDS loading buffer & Tris-HCl pH 6.8 & $200 \mathrm{mM}$ \\
& DTT & $400 \mathrm{mM}$ \\
& SDS & $8 \%(\mathrm{w} / \mathrm{v})$ \\
& Glycerol & $40 \%(\mathrm{v} / \mathrm{v})$ \\
& Bromophenol blue & $0.1 \%(\mathrm{w} / \mathrm{v})$ \\
& Prepare $1 \mathrm{ml}$ aliquots and store at $-20^{\circ} \mathrm{C}$. \\
& & $2 \mathrm{M}$ \\
$10 \times$ SDS running buffer & Glycine & $250 \mathrm{mM}$ \\
& Tris & $1 \%(\mathrm{w} / \mathrm{v})$ \\
& SDS & \\
& Tris & $1 \mathrm{M}$ \\
& Boric acid & $1 \mathrm{M}$ \\
& pH & 8.3 \\
& & $3 \mathrm{M}$ \\
& NaCl & $200 \mathrm{mM}$ \\
& Tris- $\mathrm{HCl} \mathrm{pH} 8.0$ & $1 \%(\mathrm{v} / \mathrm{v})$ \\
\hline
\end{tabular}




\begin{tabular}{|c|c|c|}
\hline Alkaline Phosphatase (AP) buffer & $\begin{array}{l}\text { Tris-HCl pH } 9.5 \\
\mathrm{NaCl} \\
\mathrm{MgCl}_{2}\end{array}$ & $\begin{array}{l}100 \mathrm{mM} \\
100 \mathrm{mM} \\
50 \mathrm{mM}\end{array}$ \\
\hline Coomassie staining solution & $\begin{array}{l}\text { Ethanol } \\
\text { Acetic acid } \\
\mathrm{ddH}_{2} \mathrm{O} \\
\text { Coomassie Brilliant Blue R-250 }\end{array}$ & $\begin{array}{l}300 \mathrm{ml} \\
100 \mathrm{ml} \\
300 \mathrm{ml} \\
0.05 \%(\mathrm{w} / \mathrm{v})\end{array}$ \\
\hline Destaining solution & $\begin{array}{l}\text { Ethanol } \\
\text { Acetic acid } \\
\text { dd }_{2} \mathrm{O}\end{array}$ & $\begin{array}{l}300 \mathrm{ml} \\
100 \mathrm{ml} \\
300 \mathrm{ml}\end{array}$ \\
\hline TBS-T + milk powder & $\begin{array}{l}20 \times \text { TBS-T } \\
\text { Skimmed Milk Powder } \\
\mathrm{ddH}_{2} \mathrm{O}\end{array}$ & $\begin{array}{l}50 \mathrm{ml} \\
40 \mathrm{~g} / 1 \\
\text { Add. } 11\end{array}$ \\
\hline
\end{tabular}

\subsection{Methods}

\subsubsection{Methods for working with plants and plant material}

\subsubsection{Methods for working with Arabidopsis thaliana}

\subsection{Surface sterilization of Arabidopsis seeds}

\subsection{Sterilization using chlorine gas}

For seed sterilization using chlorine gas, $15 \mathrm{ml}$ sodium hypochlorite were filled into a glass beaker and placed at the bottom of a desiccator. Seed packages without paper-clip were placed on the platform of the desiccator. Next, $5 \mathrm{ml} \mathrm{HCl} \mathrm{(37 \% )} \mathrm{were} \mathrm{added} \mathrm{to} \mathrm{the} \mathrm{sodium}$ hypochlorite and the desiccator was closed with a lid. Since chlorine gas is harmful the whole procedure took place under the fume hood for at least four hours or overnight.

\subsection{Sterilization using ethanol}

The amount of Arabidopsis seeds needed was transferred into a $1.5 \mathrm{ml}$ Eppendorf reaction tube. The seeds were washed three times for 2 min with $1 \mathrm{ml} 70 \% \mathrm{EtOH}$ p. a. $+0.05 \%$ Tween20. Afterwards the seeds were washed two times for 1 min with $1 \mathrm{ml} 100 \%$ EtOH p.a. The seeds were poured either on a sterile filter paper under the fume hood, if seeds were used for in vitro cultures, or on filter paper if seeds were used on soil. The seeds were allowed to dry fully before further use or storage. 


\subsection{Plant growth conditions for in vitro culture}

For expression analyses or MAPK assays surface sterilized Arabidopsis seeds were sown on $1 / 2$ MS semi-solid agar plates and grown for seven days in a plant growth chamber (PERCIVAL CU-36L5; CLF Plant Climatics, Wertingen, Germany) under short day condi-conditions $\left(10 \mathrm{~h} \mathrm{light}\right.$ at $22^{\circ} \mathrm{C}$ and $14 \mathrm{~h}$ darkness at $\left.20^{\circ} \mathrm{C}\right)$. Seven-day-old seedlings were transferred to 24-well plates and grown further under the same conditions. For this, the wells were filled with $500 \mu \mathrm{l}$ liquid $1 / 2$ MS medium and two seedlings were transferred from the $1 / 2 \mathrm{MS}$ semisolid agar plate to each well under the sterile hood. After six days the $1 / 2$ MS medium was exchanged for fresh $1 / 2$ MS medium. 14 day-old seedlings were treated with chitin $(10 \mu \mathrm{g} /$ $\mathrm{ml})$, flg22 (50 nM) or medium as control for $12 \mathrm{~min}$ (MAPK assay) or $30 \mathrm{~min}$ (gene expression analysis). Four seedlings were pooled in one Eppendorf cup that was immediately shockfrozen in liquid nitrogen.

\subsection{Plant growth conditions for cultivation in soil}

The soil (Fruhstorfer Erde, Type T, Archut) for plant cultivation was steamed twice prior to use $\left(90^{\circ} \mathrm{C}\right.$ for $30 \mathrm{~min}$, allowed to cool down, then steamed again $90^{\circ} \mathrm{C}$ for $\left.30 \mathrm{~min}\right)$ to remove soil-borne pests and pathogens. Seeds were placed directly on soil and pots were transferred to growth chambers (Johnson Controls, Milwaukee, WI, USA) with short day conditions (8 h light per day, $22^{\circ} \mathrm{C} / 20^{\circ} \mathrm{C}$ day/ night, $65 \%$ relative humidity and $140 \mathrm{~mol} \mathrm{~m}^{-2} \mathrm{~s}^{-1}$ light intensity. After seven days, seedlings were pricked out.

About four to six week-old plants were used for experiments. Six to eight week-old plants were transferred from short day to long day conditions ( $16 \mathrm{~h}$ light per day, $22^{\circ} \mathrm{C} / 20^{\circ} \mathrm{C}$ day/ night, $65 \%$ relative humidity, $200 \mathrm{~mol} \mathrm{~m}^{-2} \mathrm{~s}^{-1}$ ) for seed propagation. For faster propagation, plants were directly grown under long-day conditions.

All plants grown on soil were watered with tap water every 2-3 days.

\subsection{Infection of Arabidopsis}

\subsection{Infection with Blumeria graminis f. sp. hordei (Bgh)}

$B g h$ used for infection was grown on barley (Hordeum vulgare cv. Golden Promise) plants under short day conditions ( $8 \mathrm{~h}$ light, $22^{\circ} \mathrm{C} ; 16 \mathrm{~h}$ dark, $20 \%, 70 \%$ humidity) in a PERCIVAL AR-66L3 incubator (CLF Plant Climatics, Wertingen, Germany). For infection two pots 
with barley plants that showed clear $B g h$ growth were shaken over a tray with randomized Arabidopsis plants. Phenotypes were analysed 7 dpi.

\subsection{Infection with Erysiphe cruciferarum}

E. cruciferarum used for infection was grown on broad leaved mustard (Brassica juncea var. rugose cv. Red Giant) plants under short day conditions ( $8 \mathrm{~h}$ light, $22^{\circ} \mathrm{C} ; 16 \mathrm{~h}$ dark, $20 \%, 70$ \% humidity) in PERCIVAL chambers AR-95L3 and AR-66L3 (CLF Plant Climatics, Wertingen, Germany). For infection, a tray with randomized Arabidopsis plants was placed in a cardboard box covered with a fly screen. Eight leaves with E. cruciferarum fungal growth were cut from the mustard plants and tapped on the fly screen. Phenotypes were usually analysed between 7 and 14 dpi. For seed propagation, PERCIVAL chambers were switched to long day conditions $\left(16 \mathrm{~h}\right.$ light, $22^{\circ} \mathrm{C} ; 8 \mathrm{~h}$ dark, $20 \%, 70 \%$ humidity).

\subsection{Crossing Arabidopsis thaliana}

In order to generate crosses of different Arabidopsis lines, the plants were manually crossed. To do so, closed flower buds were chosen. First, a shoot was selected and all side branches were removed to prevent confusion with non-crossed flowers. Second, sepals, petals and stamina of the maternal flower were removed until only the carpel was left. The stigma was then pollinated with single anthers from the paternal flower. Finally, the plant was allowed to develop a silique at long-day conditions.

\subsection{Agrobacterium-mediated stable transformation of Arabidopsis}

Arabidopsis plants were transformed via a floral dipping method (Clough and Bent, 1998). Usually square pots with five plants per pot were used and at least five pots were dipped per construct. Arabidopsis lines used for transformation were grown under short day conditions for 2-4 weeks and then transferred to long day to induce flowering. The first bolts were clipped to break apical dominance. 2-4 days after clipping the new bolts were ready to be transformed. A single colony of A. tumefaciens cells transformed with the construct of interest (2.2.2.5) was used to inoculate a $5 \mathrm{ml}$ pre-culture of DYT mixed with the appropriate antibiotics. The bacteria were grown at $28^{\circ} \mathrm{C}$ and $200 \mathrm{rpm}$ in the Certomat ${ }^{\circledR}$ BS- 1 incubator (Sartorius-Stedim Biotech, Göttingen, Germany) for 2 days. $3 \mathrm{ml}$ pre-culture were used to inoculate $300 \mathrm{ml}$ DYT with appropriate antibiotics as main culture. The main culture was grown overnight and the $\mathrm{OD}_{600 \mathrm{~nm}}$ was measured. Cultures with an $\mathrm{OD}_{600 \mathrm{~nm}}$ between 0.6 and 
1.2 were spun down at $4000 \mathrm{rpm}$ for $20 \mathrm{~min}$ at RT (Heraeus multifuge $3 \mathrm{SR}^{+}$, Thermo Fisher Scientific, Waltham, USA). The supernatant was discarded and the remaining pellet was resuspended in $300 \mathrm{ml} 5 \%$ sucrose solution $+0.05 \%$ Silwet L-77. Inflorescences were dipped briefly in the Agrobacterium solution for 30-60 seconds and were then stored at low light conditions under a cover for 16-24 h to maintain high humidity. Then, plants were placed into a climate chamber with long-day conditions to set seeds.

\subsection{Selection of stably transformed Arabidopsis plants}

\subsection{Basta selection on soil}

Selection of stably transformed, Basta ${ }^{\circledR}$-resistant Arabidopsis plants was performed using Basta $^{\circledR}$ solution (183 g/ 1 glufosinate, Bayer CropScience, Monheim am Rhein, Germany). For this purpose, T1 seeds were densely sown on square pots and allowed to germinate covered with a transparent lid. After germination, seedlings were sprayed with a $0.05 \%$ Basta $^{\circledR}$ solution. This was repeated three times in two-day intervals. Successfully transformed seedlings were resistant and thus survived the Basta ${ }^{\circledR}$ treatment whereas untransformed plants died. The transformants were picked and transplanted into fresh single pots.

\subsection{In vitro selection of Arabidopsis transformands}

Surface sterilized seeds were placed on $1 / 2$ MS agar plates containing suitable antibiotics or herbicides. Plates were transferred into a growth cabinet (CLF Plant Climatics, Wertingen, Germany) with short day condition (light for $10 \mathrm{~h}$ at $22^{\circ} \mathrm{C}$ and darkness for $14 \mathrm{~h}$ at $20^{\circ} \mathrm{C}$ ). Two weeks after germination resistant plants were transferred to soil.

\subsection{Selection via seed-GFP marker}

For plants with seed-GFP marker, seeds were placed on a white paper and fluorescence was screened with the fluorescence binocular (Leica M165 FC; Leica Microsystems, Wetzlar, Germany). In this work, selection via seed-GFP marker was only used for T2 plants transformed with CRISPR/ Cas 9 constructs. For this seeds that did not show green fluorescence and therefore lost the CRISPR/ Cas9 construct were picked with a wet toothpick and directly transferred to soil. 


\subsection{Chitin infiltration of Arabidopsis thaliana leaves}

For assaying phosphorylation of CERK1, detached leaves of 5-6 week-old plants were incubated in $\mathrm{ddH}_{2} \mathrm{O}$ for $2 \mathrm{~h}$ at room temperature. Afterwards, $100 \mu \mathrm{g} / \mathrm{ml}$ chitin was added to the samples. The samples were inverted gently to distribute the chitin, and then vacuuminfiltrated for $10 \mathrm{~min}$ using a plastic desiccator. Control leaves were infiltrated with water. Infiltrated leaves were incubated for $10 \mathrm{~min}$ at RT before being frozen in liquid nitrogen in $1.5 \mathrm{ml}$ Eppendorf tubes.

\subsubsection{Methods for working with Nicotiana benthamiana}

\subsection{Nicotiana benthamiana growth conditions}

$N$. benthamiana seeds were sterilized using chlorine gas and sown on square pots. Plants were directly grown in a growth chamber (Johnson Controls, Milwaukee, WI, USA) under long-day conditions $\left(16 \mathrm{~h}\right.$ light at $25^{\circ} \mathrm{C}$ and $65 \%$ rel. humidity; $8 \mathrm{~h}$ light at $22^{\circ} \mathrm{C}$ and $65 \%$ rel. humidity). About 5-week-old plants were used for transient expression studies.

\subsection{Infiltration with Agrobacterium tumefaciens}

A single colony of $A$. tumefaciens cells transformed with the construct of interest (2.2.2.5) was used to inoculate a $5 \mathrm{ml}$ pre-culture of DYT mixed with the appropriate antibiotics. The bacteria were grown at $28^{\circ} \mathrm{C}$ and $200 \mathrm{rpm}$ in the Certomat ${ }^{\circledR}$ BS-1 incubator (Sartorius-Stedim Biotech, Göttingen, Germany) for 2 days. 50-200 $\mu 1$ pre-culture were used to inoculate $6 \mathrm{ml}$ DYT with appropriate antibiotics as main culture. The main culture was grown overnight and the $\mathrm{OD}_{600 \mathrm{~nm}}$ was measured. Cultures with an $\mathrm{OD}_{600 \mathrm{~nm}}$ between 0.8 and 1.2 were used. For infiltration, the $\mathrm{OD}_{600 \mathrm{~nm}}$ of these cultures was adjusted to 0.4 or 0.2 . To do so, the calculated amount of culture was spun down at $3500 \mathrm{rpm}$ for $5 \mathrm{~min}$ at RT (Heraeus multifuge $3 \mathrm{SR}^{+}$, Thermo Fisher Scientific, Waltham, USA), the supernatant was discarded and the pellet was resuspended in 8-10 ml infiltration medium with $150 \mu \mathrm{M}$ acetosyringone. For co-infiltration experiments adjusted cultures were mixed at equal volumes. Tubes were incubated for $2 \mathrm{~h}$ at room temperature. Afterwards cultures were used to infiltrate leaves of about five-week-old $N$. benthamiana plants with a $1 \mathrm{ml}$ needle-less syringe. Infiltrated areas were marked and plants were transferred back to the growth chamber under long day conditions. 2-3 days after infiltration confocal laser scanning microscopy was performed and/ or samples for protein extraction were harvested. 


\subsubsection{Confocal Laser Scanning Microscopy}

Stable transgenic Arabidopsis plants or transiently transformed N. benthamiana leaves expressing fluorescence protein-tagged fusion proteins where analyzed via confocal laser scanning microscopy. Two different confocal microscope systems, Leica TCS SP5 and Leica TCS SP8 (Leica, Wetzlar, Germany) were used for analysis together with the appropriate software (LAS AF Leica Application Suite, Version 2.7.2 and LAS X 3.5.1.18803). Small and preferably even leaf discs were cut out and placed onto an object slide wetted with water, before the cover glass was placed on top. Analyses were performed with the appropriate lasers and emission filters. The excitation and emission spectra of the fluorophores that were used in this study are listed in table 11 .

Table 11: Parameters used for detection of the different fluorophores.

\begin{tabular}{lll}
\hline Fluorophore & Excitation & Emission \\
\hline CFP & $440 \mathrm{~nm}$ & $460-500 \mathrm{~nm}$ \\
$(\mathrm{~m})$ Citrine & $514 \mathrm{~nm}$ & $525-560 \mathrm{~nm}$ \\
mKate2 & $561 \mathrm{~nm}$ & $620-640 \mathrm{~nm}$ \\
Chlorophyll autofluorescence & & $740-770 \mathrm{~nm}$ \\
\hline
\end{tabular}

\subsubsection{Methods for working with bacteria}

\subsubsection{Cultivation of bacteria}

E. coli cells were either cultivated on solid LB plates or in liquid LB medium. Appropriate antibiotics were added as selective markers. Antibiotics used in this study are summarized in table 8. Single colonies from LB plates were used to inoculate liquid medium. E. coli cells on plates were grown at $37^{\circ} \mathrm{C}$ in an IPP 500 incubator (Memmert, Schwabach, Germany), liquid cultures were grown at $37^{\circ} \mathrm{C}$ and $220 \mathrm{rpm}$ in an Innova 4230 incubator (New Brunswick Scientific, Edison, New Jersey, USA).

A. tumefaciens GV3101 (pMP90, pMP90RK or pSoup) cells used for transformation of Arabidopsis plants or infiltration of $N$. benthamiana plants were cultivated in liquid DYT medium supplied with the respective antibiotics (Table 8) for selection or on the corresponding DYT agar plates. Agrobacteria were grown for 2-3 days at $28{ }^{\circ} \mathrm{C}$ in an IPP 500 incubator (Memmert, Schwabach, Germany) and liquid cultures were additionally shaken at $200 \mathrm{rpm}$ in a CERTOMAT ${ }^{\circledR}$ BS-1 incubator (Sartorius-Stedim Biotech, Göttingen, Germany). 


\subsubsection{Preparation of chemically competent $E$. coli cells}

Six single colonies of $E$. coli TOP10 cells were used to inoculate 6 × $3 \mathrm{ml} \mathrm{LB}$ medium. The cultures were grown overnight at $37^{\circ} \mathrm{C}$ while shaking. The next day, $1 \mathrm{ml}$ of every pre-culture was used to inoculate $100 \mathrm{ml}$ of $\mathrm{LB}+10 \mathrm{mM} \mathrm{MgCl}_{2}+10 \mathrm{mM} \mathrm{MgSO}_{4}$. Cultures were grown at $37^{\circ} \mathrm{C}$ and $260 \mathrm{rpm}$ for about $2 \mathrm{~h}$ until $\mathrm{OD}_{600 \mathrm{~nm}}=0.4-0.6$ was reached. The culture was cooled for $20 \mathrm{~min}$ on ice and then divided into pre-chilled $50 \mathrm{ml}$ tubes. The tubes were spun down in a swing out centrifuge (Heraeus multifuge 3SR+, Thermo Fisher Scientific, Waltham, USA) at $3000 \mathrm{rpm}$ and $4^{\circ} \mathrm{C}$ for $15 \mathrm{~min}$. All following steps were carried out on ice. The supernatant was discarded and the remaining pellets were re-suspended in $8.3 \mathrm{ml}$ ice-cold RF I solution per tube. Three cultures were pooled and incubated at least $30 \mathrm{~min}$ on ice. Afterwards the cells were spun down at $3000 \mathrm{rpm}$ and $4{ }^{\circ} \mathrm{C}$ for $15 \mathrm{~min}$ and the supernatant was removed. Pellets were resuspended in $3 \mathrm{ml}$ ice-cold RF II solution and again incubated on ice for at least $15 \mathrm{~min}$. Finally, the cells were aliquoted (30 $\mu$ l) into pre-cooled Eppendorf cups and frozen in liquid nitrogen before being stored at $-80^{\circ} \mathrm{C}$.

\subsubsection{Preparation of electro-competent $A$. tumefaciens cells}

$50 \mathrm{ml}$ DYT medium supplemented with the appropriate antibiotics were inoculated with an $A$. tumefaciens colony from a DYT plate. The culture was incubated at $28^{\circ} \mathrm{C}$ and $200 \mathrm{rpm}$ for two days. The next day, $250 \mathrm{ml}$ DYT containing the appropriate antibiotics were inoculated to an $\mathrm{OD}_{600 \mathrm{~nm}}=0.3$ and the cells were grown at $28^{\circ} \mathrm{C}$ and $200 \mathrm{rpm}$ to an $\mathrm{OD}_{600 \mathrm{~nm}}=1.2$. The cultures were spun down at $4^{\circ} \mathrm{C}$ and $4500 \mathrm{~g}$ for $30 \mathrm{~min}$ (Heraeus multifuge $3 \mathrm{SR}+$, Thermo Fisher Scientific, Waltham, USA). The supernatant was removed and the pellet was resuspended in $30 \mathrm{ml}$ ice-cold $1 \mathrm{mM}$ HEPES, pH 7.0. These steps were repeated twice. After removal of the supernatant, the pellet was resuspended in $30 \mathrm{ml}$ ice-cold $10 \%$ glycerol and centrifuged as before. The supernatant was removed and the pellet was resuspended in $2 \mathrm{ml}$ ice-cold $10 \%$ glycerol. The cells were divided into $50 \mu \mathrm{l}$ aliquots in $1.5 \mathrm{ml}$ reaction tubes and frozen in liquid nitrogen before being stored at $-80^{\circ} \mathrm{C}$.

\subsubsection{Transformation of chemically competent $E$. coli cells}

One aliquot E. coli TOP10 competent cells was thawed on ice. 5-10 $\mu 1$ of a ligation or $0.5-1$ $\mu 1$ plasmid DNA were added to the cells and mixed well. The cells were incubated on ice for $20 \mathrm{~min}$. After that, the cells were heat-shocked for $30-45 \mathrm{~s}$ at $42^{\circ} \mathrm{C}$. Then $600 \mu \mathrm{LB}$ were added and the cells were incubated for $60 \mathrm{~min}$ at $37^{\circ} \mathrm{C}$ and $650 \mathrm{rpm}$. After incubation, the 
cells were spun down in a table top centrifuge (Heraeus Pico21, Thermo Fisher Scientific, Waltham, USA) for $1 \mathrm{~min}$ at $14000 \mathrm{rpm}$ and nearly all supernatant was discarded. The pellet was resuspended in the remaining supernatant and the solution was then streaked out on LB with according antibiotics. LB plates were incubated overnight at $37^{\circ} \mathrm{C}$.

\subsubsection{Transformation of electro-competent $A$. tumefaciens cells}

An aliquot of competent Agrobacterium cells was thawed on ice. $0.5 \mu 1$ plasmid DNA were added to the cells and mixed well. The mixture was transferred into a precooled sterile electroporation cuvette with $0.1 \mathrm{~cm}$ gap width. Electroporation was performed using a Micro Pulser $^{\mathrm{TM}}$ (BioRad, München, Germany) apparatus (setting: $25 \mu \mathrm{F}, 2.5 \mathrm{kV}$ and $400 \Omega$ ). The cuvette was transferred back on ice and $600 \mu$ liquid DYT were added. The bacterial solution was transferred into a sterile $1.5 \mathrm{ml}$ reaction tube and the sample was incubated at $28^{\circ} \mathrm{C}$ and $200 \mathrm{rpm}$ for $2 \mathrm{~h}$. Then $100 \mu \mathrm{l}$ of the mixture were plated onto a DYT agar plate with the appropriate antibiotics and the plate was incubated at $28^{\circ} \mathrm{C}$ for $2-3$ days.

\subsubsection{Storage of bacterial cultures}

Short-term storage of bacteria is possible by keeping the cells on solid medium sealed with parafilm ${ }^{\circledR}$. The cells are viable for up to one month at $4^{\circ} \mathrm{C}$. For long-term storage, glycerol stocks of the respective cells were made by mixing $1 \mathrm{ml}$ overnight culture with $1 \mathrm{ml}$ sterile 65 $\%$ glycerol. The cells were subsequently stored at $-80^{\circ} \mathrm{C}$.

\subsubsection{Molecular biological methods}

\subsubsection{Isolation of genomic DNA (gDNA) from Arabidopsis thaliana}

\subsection{Isolation of gDNA for PCR amplification}

For fast and simple gDNA isolation one small leave of the plant center was harvested in a 1.5 $\mathrm{ml}$ tube and either directly used for DNA extraction, stored up to two days at $-20^{\circ} \mathrm{C}$ or frozen in liquid nitrogen for long time storage at $-80^{\circ} \mathrm{C}$.

The DNA quickprep buffer was shortly heated in the microwave to dissolve the precipitate and $300 \mu \mathrm{l}$ buffer were added to each sample. The leaves were ground with a plastic pistil using the IKA ${ }^{\circledR}$ RW20 digital drill (IKA-Werke, Staufen, Germany) and the mixture was incubated afterwards in a thermomixer at $600 \mathrm{rpm}$ and RT for $5 \mathrm{~min}$. Afterwards cell fragments were pelleted by 5 min centrifugation at 13000 rpm (Heraeus Pico21, Thermo Fisher Scien- 
tific, Waltham, USA). $240 \mu \mathrm{l}$ of the supernatant were transferred into a new $1.5 \mathrm{ml}$ tube filled with $300 \mu \mathrm{l}$ isopropanol. The tube was incubated for $5 \mathrm{~min}$ and $600 \mathrm{rpm}$ in a thermomixer at RT and then centrifuged again for $5 \mathrm{~min}$ at $13000 \mathrm{rpm}$. The supernatant was discarded and the formed DNA pellet was washed with $1 \mathrm{ml} 70 \%$ ethanol. The pellet was then air-dried at $40^{\circ} \mathrm{C}$ or RT and resuspended in $50 \mu 1 \mathrm{ddH}_{2} \mathrm{O} .1 \mu \mathrm{l}$ of this DNA was typically used for a $25 \mu \mathrm{l}$ PCR reaction.

\subsection{Isolation of gDNA for Next Generation Sequencing (NGS)}

To isolate DNA of a higher quality necessary for NGS, the DNeasy ${ }^{\circledR}$ Plant Maxi Kit (Qiagen, Hilden, Germany) was used as described in the user's manual if not stated otherwise. About $0.8 \mathrm{~g}$ of pulverized frozen plant material was used for DNA extraction and the elution was

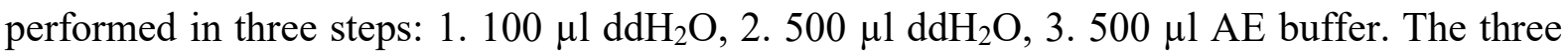
eluates were kept separate and the one with the highest DNA concentration and purity was used for NGS.

\subsubsection{Isolation of plasmid DNA from E. coli}

\subsection{Small-scale plasmid preparation (Birnboim and Doly, 1979)}

$1.5 \mathrm{ml}$ of liquid bacterial overnight culture were transferred into a $1.5 \mathrm{ml}$ reaction tube and spun down for $1 \mathrm{~min}$ at $13000 \mathrm{rpm}$ in a table top centrifuge (Heraeus Pico21, Thermo Fisher Scientific, Waltham, USA). After removal of the supernatant the remaining pellet was resuspended in $200 \mu \mathrm{l}$ buffer P1. Next, $200 \mu \mathrm{l}$ buffer P2 were added and mixed gently by inverting the tube. The samples were incubated for 3-5 min at room temperature. The lysis reactions were then stopped by adding $200 \mu \mathrm{l}$ buffer P3 and immediately mixed by inverting several times. After that, the samples were centrifuged for $10 \mathrm{~min}$ at $13000 \mathrm{rpm}$. Then, $500 \mu 1$ of the clear supernatant were transferred into a new $1.5 \mathrm{ml}$ reaction tube containing $1 \mathrm{ml} 96$ $\%$ ethanol p.a. without disturbing the white precipitate. Then, the tubes were centrifuged for 5 min at $13000 \mathrm{rpm}$. The supernatant was removed and the formed DNA pellet was washed with $70 \%$ ethanol and centrifuged for $1 \mathrm{~min}$ at $13000 \mathrm{rpm}$. After removing the supernatant and an additional centrifugation step for $1 \mathrm{~min}$ at $13000 \mathrm{rpm}$, the remaining ethanol was removed and the formed pellet was air-dried at room temperature. Finally, the pellet was resuspended in $50 \mu \mathrm{ldd} \mathrm{H}_{2} \mathrm{O}$. 


\subsection{Medium-scale plasmid preparation}

To reach a higher yield and purity of DNA, $50 \mathrm{ml}$ overnight culture were spun down and used for DNA isolation with the Plasmid Plus Midi Kit (Qiagen, Hilden, Germany) according to the manufacturer's instructions.

\subsubsection{Isolation of plasmid DNA from A. tumefaciens}

For isolation of plasmid DNA from A. tumefaciens, $200 \mu \mathrm{l}$ of a culture grown for two days at $28^{\circ} \mathrm{C}$ and $200 \mathrm{rpm}$ were spun down at $13000 \mathrm{rpm}$ for $1 \mathrm{~min}$ (Heraeus Pico21, Thermo Fisher Scientific, Waltham, USA). The supernatant was discarded and the pellet was used for DNA isolation as described in 2.2.3.2.1.

\subsubsection{Polymerase chain reaction (PCR)}

\subsection{Standard PCR}

For standard applications such as genotyping, PCR was performed with homemade Taq polymerase and either gDNA or plasmid DNA as template. PCRs were performed in the PCR cyclers MyCycler ${ }^{\mathrm{TM}}$ and $\mathrm{T} 100^{\mathrm{TM}}$ (Bio-Rad, Munich, Germany).

$\begin{array}{lll}\text { PCR mix for one reaction: } & \text { 10x reaction buffer } & 2.5 \mu \mathrm{l} \\ & \text { primer } 1(10 \mu \mathrm{M}) & 0.5 \mu \mathrm{l} \\ & \text { primer } 2(10 \mu \mathrm{M}) & 0.5 \mu \mathrm{l} \\ & \text { dNTPs }(10 \mathrm{mM}) & 0.5 \mu \mathrm{l} \\ & \text { Taq Polymerase } & 0.1 \mu \mathrm{l} \\ \text { template DNA } & 1.0 \mu \mathrm{l} \\ \mathrm{ddH}_{2} \mathrm{O} & 19.9 \mu \mathrm{l}\end{array}$

\begin{tabular}{|c|c|c|c|}
\hline PCR program: & Initial denaturation & $94^{\circ} \mathrm{C}$ & $5 \mathrm{~min}$ \\
\hline & Denaturation & $94^{\circ} \mathrm{C}$ & $30 \mathrm{sec}$ \\
\hline & Annealing & $55-62^{\circ} \mathrm{C}$ & $30 \mathrm{sec}$ \\
\hline & Elongation & $72^{\circ} \mathrm{C}$ & $1 \mathrm{~min} / \mathrm{kb}$ \\
\hline & Final elongation & $72^{\circ} \mathrm{C}$ & $10 \mathrm{~min}$ \\
\hline & & $4^{\circ} \mathrm{C}$ & $\infty$ \\
\hline
\end{tabular}

The annealing temperature was adjusted to the primers used and the extension time to the length of the fragment to be amplified. 


\subsection{PCR for generation of DNA fragments used for cloning}

For DNA fragments used for cloning, an accurate dsDNA synthesis is necessary. Therefore, proofreading polymerases with a low error frequency are used. Here the iProof ${ }^{\mathrm{TM}}$ High Fidelity PCR kit (Bio-Rad, Munich, Germany) was used according to the user's manual.

\begin{tabular}{|c|c|c|c|c|}
\hline PCR mix for one reaction: & 10x HF buffer & $10 \mu \mathrm{l}$ & & \\
\hline & primer $1(10 \mu \mathrm{M})$ & $2.5 \mu \mathrm{l}$ & & \\
\hline & primer $2(10 \mu \mathrm{M})$ & $2.5 \mu \mathrm{l}$ & & \\
\hline & dNTPs $(10 \mathrm{mM})$ & $0.4 \mu \mathrm{l}$ & & \\
\hline & iproof Polymerase & $0.5 \mu \mathrm{l}$ & & \\
\hline & template DNA & $1.0 \mu \mathrm{l}$ & & \\
\hline & $\mathrm{ddH}_{2} \mathrm{O}$ & $33.1 \mu 1$ & & \\
\hline PCR program: & Initial denaturation & $98^{\circ} \mathrm{C}$ & $5 \mathrm{~min}$ & \\
\hline & Denaturation & $98^{\circ} \mathrm{C}$ & $10 \mathrm{sec}$ & \\
\hline & Annealing & $55-62^{\circ} \mathrm{C}$ & $30 \mathrm{sec}$ & $35 \mathrm{x}$ \\
\hline & Elongation & $72^{\circ} \mathrm{C}$ & $30 \mathrm{sec} / \mathrm{kb}$ & \\
\hline & Final elongation & $72^{\circ} \mathrm{C}$ & $10 \mathrm{~min}$ & \\
\hline & & $4^{\circ} \mathrm{C}$ & $\infty$ & \\
\hline
\end{tabular}

\subsubsection{DNA agarose gel electrophoresis}

In order to separate and visualize DNA fragments, samples were mixed with 6x DNA loading dye and separated in a 1-3\% agarose gel by gel electrophoresis. For gel preparation the respective amount of agarose was mixed with 1x TAE buffer and heated in a microwave until all of the agarose was dissolved. Then the mix was allowed to cool down to about $50^{\circ} \mathrm{C}$ and 5 $\mu 1$ HDgreen $^{\mathrm{TM}}$ (Intas, Göttingen, Germany) were added per $100 \mathrm{ml}$. The solid gel was then transferred into a Sub-Cell GT tank (BioRad, Munich, Germany) and the tank was filled with 1x TAE buffer. The samples and GeneRuler ${ }^{\mathrm{TM}} 1 \mathrm{~kb}, 100 \mathrm{bp}$ plus or $50 \mathrm{bp}$ DNA ladder (Thermo Fisher Scientific, Waltham, USA) were loaded into the wells. The gel was run at a voltage of 90-120 V for 30-50 min and afterwards analyzed with a Genoplex Transilluminator (UV at $312 \mathrm{~nm}$ ) gel documentation and analysis system (VWR, Radnor, Pennsylvania, USA). 


\subsubsection{Purification of DNA fragments}

PCR products and DNA fragments for cloning and sequencing were either cleaned-up directly or after gel electrophoresis. For the latter, gel slices were cut out under UV-light (365 nm) for visualization using a scalpel and transferred to a $1.5 \mathrm{ml}$ reaction tube. For gel-elution and PCR product purification the NucleoSpin ${ }^{\circledR}$ Gel and PCR Clean-up kit (Macherey-Nagel, Düren, Germany) was used according to the manufacturer's instructions.

\subsubsection{Photometric measurement of DNA and RNA concentration}

For determination of DNA and RNA concentrations as well as for checking the purity of the nucleic acids, the TECAN Infinite ${ }^{\circledR}$ M200 plate reader (Tecan Group Ltd, Männedorf, Switzerland) was used. The NanoQuant Plate ${ }^{\mathrm{TM}}$ was blanked first and then $2 \mu \mathrm{l}$ of the sample were pipetted onto the plate wells. The absorption was measured at $260 \mathrm{~nm}$ and $280 \mathrm{~nm}$. The ratio between the absorbance of $260 \mathrm{~nm}$ and $280 \mathrm{~nm}$ indicates the purity of the sample. The optimal ratio $\left(\mathrm{OD}_{260 \mathrm{~nm}} / \mathrm{OD}_{280 \mathrm{~nm}}\right)$ for $\mathrm{DNA}$ is $\sim 1.8$ and for RNA $\sim 2.0$.

\subsubsection{Restriction enzyme digestion of DNA}

The used restriction enzymes were standard or FastDigest ${ }^{\circledR}$ enzymes from Thermo Fisher Scientific (Waltham, USA) and were used according to the manufacturer's instructions. For normal restriction digestion reactions $2 \mu 1$ 10x reaction buffer were mixed with 2-5 units of the respective restriction endonuclease and $1 \mu \mathrm{g}$ DNA. This mix was then filled up with water to $20 \mu \mathrm{l}$. The reaction was incubated at the appropriate temperature for $30 \mathrm{~min}$ (FastDigest ${ }^{\circledR}$ ) or $4 \mathrm{~h}$ to overnight (standard enzymes). Digestion products were analyzed by agarose gel electrophoresis. Restriction digestion was used for genotyping, cloning and analysis of plasmids.

\subsubsection{Dephosphorylation of plasmid DNA}

Vector fragments with matching ends were dephosphorylated to avoid re-circulation during ligation steps. Up to $5 \mu \mathrm{g}$ DNA were used in a $50 \mu$ d dephosphorylating reaction using shrimp alkaline phosphatase (SAP, $1 \mathrm{U} / \mu \mathrm{l}$ ) (Thermo Fisher Scientific, Waltham, USA). 
Dephosphorylation mix for one reaction:

$\begin{array}{lc}\text { DNA } & \leq 5 \mu \mathrm{g} \\ 10 \times \text { SAP reaction buffer } & 5 \mu \mathrm{l} \\ \text { SAP }(1 \mathrm{U} / \mu \mathrm{l}) & 1 \mu \mathrm{l} \\ \text { Add } \mathrm{ddH}_{2} \mathrm{O} \text { to } 50 \mu \mathrm{l} & \end{array}$

The depohosphorylation mix was incubated for $1 \mathrm{~h}$ at $37^{\circ} \mathrm{C}$. SAP was then inactivated by incubating the sample at $72^{\circ} \mathrm{C}$ for $20 \mathrm{~min}$.

\subsubsection{Ligation of DNA fragments}

Ligation was performed using the T4 DNA ligase (Thermo Fisher Scientific, Waltham, USA). Vector backbones and inserts were cut with matching restriction enzymes and mixed at a molar ratio of 1:3. The molar ratio was calculated using the NEBioCalculator ${ }^{\mathrm{TM}} \mathrm{v} 1.10 .1$ (NEB, Ipswich, Massachusetts, USA). Ideally, $\geq 100 \mu \mathrm{g}$ vector fragment were used and the ligation mix was pipetted as described below. Ligation was performed at RT for $4 \mathrm{~h}$ or at $16^{\circ} \mathrm{C}$ overnight. Up to $10 \mu$ ligation reaction were then used for transformation of $E$. coli cells.

Ligation mix for one reaction: Vector fragment $\quad \geq 100 \mu \mathrm{g}$

Insert fragment (molar ratio vector to insert 3:1) $\mathrm{x} \mu \mathrm{g}$

$10 \mathrm{x}$ T4 ligase reaction buffer $\quad 2 \mu 1$

T4 DNA ligase $\quad 1 \mu \mathrm{l}(5 \mathrm{U})$

Add $\mathrm{ddH}_{2} \mathrm{O}$ to $20 \mu \mathrm{l}$

\subsubsection{Q5 site directed mutagenesis}

For site directed mutagenesis the Q $5^{\circledR}$ Site-Directed Mutagenesis Kit (New England Biolabs, Ipswich, Massachusetts, USA) was used according to the manufacturer's instructions.

\subsubsection{Sequencing of DNA}

\subsection{Sequencing of plasmids and PCR products}

Plasmids and PCR products were sequenced by Microsynth Seqlab (Göttingen, Germany). Plasmids were adjusted to $40-100 \mathrm{ng} / \mu \mathrm{l}$ and PCR products to $1.5 \mathrm{ng}$ per $100 \mathrm{bp}$ PCR product in a volume of $12 \mu 1.3 \mu \mathrm{l}$ primer $(10 \mu \mathrm{M})$ were added to every sample. 


\subsection{Next generation sequencing}

For next generation sequencing DNA was extracted as described in 2.2.3.1.2 and either sent lyophilized (Novogene) or dissolved in $\mathrm{ddH}_{2} \mathrm{O}$ (GATC, TAL).

$50 \mathrm{bp}$ Illumina reads were generated by the Transcriptome and Genome Analysis Laboratory (TAL; Göttingen, Germany). For 150 bp paired-end sequencing, DNA samples were sent either to GATC (Constance, Germany) or Novogene (Beijing, China or Cambridge, UK).

\subsubsection{RNA isolation from plants}

If plant pools were used for RNA extraction, the plant material was coarsely ground with mortar and pestle in liquid nitrogen and $80-90 \mathrm{mg}$ frozen powder were weighed out into $2 \mathrm{ml}$ safe-seal Eppendorf tubes. Afterwards one $5 \mathrm{~mm}$ and two $3 \mathrm{~mm}$ steal balls were added to every tube and the material was homogenized using the TissueLyser LT (Qiagen, Hilden, Germany) three times for $1 \mathrm{~min}$. In between the homogenizing steps the samples were cooled in liquid nitrogen.

If RNA was extracted from single plants or seedlings, the frozen material was directly homogenized with the TissueLyser LT four times as described.

The homogenized plant material was used for RNA extraction with the innuPREP Plant RNA Kit (Analytik Jena, Jena, Germany) as described in the user's manual. RNA was eluted in 55 $\mu 1$ RNase free water and always kept on ice. RNA concentration was measures with the TECAN Infinite ${ }^{\circledR}$ M200 plate reader (Tecan Group Ltd, Männedorf, Switzerland) and adjusted to $0.09 \mu \mathrm{g} / \mu \mathrm{l} .10 \mu \mathrm{l}$ adjusted RNA was loaded on a $2 \%$ agarose gel to investigate RNA quality and equality.

\subsubsection{Synthesis of complementary DNA (cDNA)}

For cDNA synthesis, $11.5 \mu \mathrm{l}(\hat{=} 1 \mu \mathrm{g})$ adjusted RNA isolated as described in 2.2.3.13 were mixed with $1 \mu 1100 \mu \mathrm{M}$ oligodT primer. This mixture was incubated for $5 \mathrm{~min}$ at $65^{\circ} \mathrm{C}$, then transferred to ice. For cDNA synthesis, $7.5 \mu 1$ master mix were added to every sample and cDNA was amplified using the PCR program shown below.

\begin{tabular}{|c|c|}
\hline cDNA master mix for one reaction: & 5x Reverse Transcriptase (RT) buffer \\
\hline & Ribolock \\
\hline & $10 \mathrm{mM}$ dNTPs \\
\hline & Reverse Transcriptase \\
\hline
\end{tabular}




$\begin{array}{lll}\text { Program for cDNA synthesis: } & 42^{\circ} \mathrm{C} & 60 \mathrm{~min} \\ & 70^{\circ} \mathrm{C} \quad 10 \mathrm{~min} \\ & 4^{\circ} \mathrm{C} \quad \infty\end{array}$

\subsubsection{Semi-quantitative reverse transcription PCR (Semi-qRT-PCR)}

cDNA was generated as described in 2.2.3.14 and diluted 1:5 (10 $\mu 1$ undiluted cDNA $+40 \mu 1$ $\mathrm{ddH}_{2} \mathrm{O}$ ) and 1:15 ( $20 \mu \mathrm{l}$ cDNA 1:5 diluted $+40 \mu 1 \mathrm{ddH}_{2} \mathrm{O}$ ). $3 \mu 1$ 1:15 diluted cDNA were used for PCR with homemade Taq polymerase.

semi-qRT-PCR mix for one reaction:

$\begin{array}{ll}\text { cDNA } 1: 15 & 3 \mu \mathrm{l} \\ \text { 10x Taq buffer } & 2.5 \mu \mathrm{l} \\ \text { primer } 1(10 \mu \mathrm{M}) & 0.5 \mu \mathrm{l} \\ \text { primer } 2(10 \mu \mathrm{M}) & 0.5 \mu \mathrm{l} \\ \text { dNTPs }(10 \mathrm{mM}) & 0.5 \mu \mathrm{l} \\ \text { Taq Polymerase } & 0.1 \mu \mathrm{l} \\ \mathrm{ddH}_{2} \mathrm{O} & 17.9 \mu \mathrm{l}\end{array}$

semi-qRT-PCR program:

Initial denaturation
Denaturation
Annealing
Elongation
Final elongation

$\left.\begin{array}{ll}94^{\circ} \mathrm{C} & 5 \mathrm{~min} \\ 94^{\circ} \mathrm{C} & 30 \mathrm{sec} \\ 55-62^{\circ} \mathrm{C} & 30 \mathrm{sec} \\ 72^{\circ} \mathrm{C} & 1 \mathrm{~min} / \mathrm{kb}\end{array}\right] 24-35 \mathrm{x}$

The annealing temperature was adjusted to the primers used and the extension time to the length of the fragment to be amplified. PCR cycles were adapted to the amplified transcripts.

\subsubsection{Quantitative reverse transcription PCR (qRT-PCR)}

For qRT-PCR, the amplification and simultaneous quantification was carried out with the CFX96 Touch TM Real-Time PCR Detection System with the CFX Manager TM Software and suitable qRT-PCR-96-well plates (BioRad, Hercules, CA, USA). Reactions were set up with Sso Fast EvaGreen supermix (BioRad, Munich, Germany) with four technical replicates per sample. 
qRT-PCR mix for one reaction:

$\begin{array}{ll}\text { cDNA 1:500 } & 3 \mu 1 \\ \text { EvaGreen supermix } & 5 \mu 1 \\ \text { Primer mix (2 } \mu \mathrm{M} \text { each }) & 2 \mu 1\end{array}$

qRT-PCR program:

$\left.\begin{array}{lll}\text { Denaturation } & 95^{\circ} \mathrm{C} & 30 \mathrm{~s} \\ \text { Annealing } & 95^{\circ} \mathrm{C} & 5 \mathrm{~s} \\ \text { Extension } & 55^{\circ} \mathrm{C} & 10 \mathrm{~s}\end{array}\right] 45 \mathrm{x}$

\subsubsection{ROS Burst assay}

To analyze the production of reactive oxygen species (ROS) upon PAMP treatment in Arabidopsis, a chemiluminescence-based assay was performed using 96-well plates.

Each well was filled with $100 \mu$ tap water. Leaf discs of 5-7 week-old plants with a diameter of $4 \mathrm{~mm}$ were harvested and transferred into the wells. The plate was wrapped into a plastic bag und incubated overnight at room temperature.

The next day, the water in the wells was removed carefully and replaced with either $100 \mu 1$ freshly prepared L-012 solution, L-012 solution with chitin $(100 \mu \mathrm{g} / \mathrm{ml})$ or L-012 solution with flg22 (100 nM). The L-012 solution was added to the leaf discs directly before starting the measurement. Chemiluminescence was measured every minute over a period of $1 \mathrm{~h}$ using a TECAN infinite ${ }^{\circledR}$ M200 plate reader (Tecan Group Ltd, Männedorf, Switzerland). The obtained data were analysed with Excel (Microsoft Corporation, Albuquerque, New Mexico, USA).

\subsubsection{Biochemical methods}

\subsubsection{Protein extraction and purification from plants}

\subsection{Small scale total protein extraction}

As starting material either 80-90 mg frozen plant powder or the same amount of frozen leaf material were used. All samples were weighed out in $1.5 \mathrm{ml}$ Eppendorf cups and the samples were kept on ice at all times to prevent protein degradation. $200 \mu$ CERK1 extraction buffer + Protease Inhibitor Cocktail (PIC, 1:100) and one little spoon of sea sand were added. Afterwards samples were directly ground with a glass pistil at 1000 rounds/ min for 30-60 s 
using the IKA ${ }^{\circledR}$ RW20 digital drill (IKA-Werke, Staufen, Germany). $200 \mu$ l CERK1 extraction buffer + PIC were used to rinse the pistil and collected in the tube. Another $800 \mu 1$ CERK1 extraction buffer + PIC were directly added to the tube, making $1200 \mu 1$ in total.

For protein extraction from Arabidopsis seedlings grown in in vitro, protein extraction was performed in a total volume of $600 \mu 1$ CERK1 extraction buffer + PIC.

The pistil was washed with water and dried before the next sample was ground. Homogenized samples were centrifuged for $6 \mathrm{~min}$ at $13000 \mathrm{rpm}$ and $4^{\circ} \mathrm{C}$ (Heraeus Fresco 17; Thermo Fisher Scientific, Waltham, USA). The supernatants (= total protein extracts) were transferred to new tubes. Protein concentration was determined via Bradford assay (2.2.5.3). The concentrations were adjusted to $1-2 \mu \mathrm{g} / \mathrm{ml}$. Usually $25 \mu \mathrm{l}$ of the adjusted protein extract were then mixed with $75 \mu 14 x$ SDS buffer and stored at $-20^{\circ} \mathrm{C}$ until further use.

\subsection{Large scale total protein extraction}

Two to five $\mathrm{ml}$ frozen plant powder were used as starting material for protein extraction for proteomics and in vitro kinase assay experiments. The powder was pestled in liquid nitrogen until super fine powder was obtained. The mortar with perfectly ground powder was set aside. $2 \mathrm{ml} \mathrm{CERK1} \mathrm{extraction} \mathrm{buffer} \mathrm{per} \mathrm{ml}$ powder were added to the mortar as soon as the powder started to thaw (dark green color of the sample at the edge of the mortar). The sample was mixed using the pestle until the sample was fully thawed. Then, the sample was transferred to a $50 \mathrm{ml}$ tube on ice. The mortar was rinsed twice, first with $1 \mathrm{ml}$ CERK1 extraction buffer per $\mathrm{ml}$ starting material, then with $0.2 \mathrm{ml}$ CERK1 extraction buffer per $\mathrm{ml}$ starting material. All sample fractions were combined in the tube and centrifuged for 5 min at $4000 \mathrm{rpm}$ and $4{ }^{\circ} \mathrm{C}$ (Heraues multifuge $3 \mathrm{SR}^{+}$; thermos Fisher Scientific, Waltham, USA). The supernatant (= total protein extract) was filtered through a CellTrics filter $(50 \mu \mathrm{M}$, Sysmex, Kōbe, Japan) and the protein concentration was measured via Bradford assay (2.2.4.2) and adjusted.

\subsubsection{Protein concentration measurement via the Bradford assay}

Protein concentration determination was carried out according to Bradford (1976). First, the Bradford reagent (Roti ${ }^{\circledR}$-Quant, Roth, Karlsruhe, Germany) was diluted 1:5 in $\mathrm{ddH}_{2} \mathrm{O}$. Then, $0,3,5,7,10$ and $15 \mu \mathrm{g}$ bovine serum albumin (BSA) was pipetted into cuvettes. Next, $3 \mu \mathrm{l}$ of each total extract was pipetted into cuvettes in duplicates. $1 \mathrm{ml}$ 1:5 diluted Bradford reagent was added to every cuvette and mixed well by vortexing. After 10 min incubation at RT the absorbance was measured at $595 \mathrm{~nm}$ using a WPA Biowave II photometer (Biochrom AG, Berlin, Germany). By plotting A $595 n$ of the BSA standards against their concentration, a 
standard curve was generated, which was used to calculate the protein concentration of the samples. To equalize protein concentrations, the samples were adjusted to the sample with the lowest protein concentration, ideally 1-2 $\mu \mathrm{g} / \mu \mathrm{l}$, using CERK1 extraction buffer.

\subsubsection{Protein pull-down from total protein extracts}

\subsection{Protein pull-down for co-immunoprecipitation experiments}

For co-immunoprecipitation experiments, GFP-Trap agarose beads (ChromoTek, Martinsried, Germany) and FLAG-agarose beads (Sigma-Aldrich, Munich, Germany) were prepared by washing $3 \mathrm{x}$ with $500 \mu \mathrm{l}$ CERK1 extraction buffer with a total DTT concentration of $3 \mathrm{mM}$. To pellet the beads in between the washing steps, tubes were centrifuged for $1 \mathrm{~min}$ at $500 \mathrm{rpm}$ in a Heraeus Fresco 17 centrifuge (Thermo Fisher Scientific, Waltham, USA). After washing, $15 \mu \mathrm{l}$ GFP-Trap agarose beads or $40 \mu \mathrm{l}$ of FLAG-beads were added to the protein extracts (containing about $1 \mathrm{mg}$ protein in total). The samples were incubated on a wheel at $20 \mathrm{rpm}$ and $4^{\circ} \mathrm{C}$ for $4 \mathrm{~h}$ and afterwards pelleted by centrifuging $1 \mathrm{~min}$ at $500 \mathrm{rpm}$. The supernatant was discarded and the beads were washed five times with $1 \mathrm{ml}$ cold TBS-T. Next, beads were dried with an insulin syringe and resuspended in $50 \mu 11 \mathrm{x}$ SDS loading dye. The samples were stored at $-20^{\circ} \mathrm{C}$ until further use.

\subsection{Protein pull-down for proteomics experiments and in vitro kinase assays}

For proteomics experiments and in vitro kinase assays, $50 \mu 1$ GFP-Trap agarose beads were washed four times with $250 \mu$ l CERK1 extraction buffer as described in 2.2.5.3.1 and transferred to the protein extracts containing 10-20 mg protein. The samples were incubated on a wheel at $20 \mathrm{rpm}$ and $4^{\circ} \mathrm{C}$ for $4 \mathrm{~h}$. Afterwards the experiments were continued as described in 2.2.4.6 (in vitro kinase assays) or 2.2.4.9 (proteomics experiments).

\subsubsection{SDS-polyacrylamide gel electrophoresis (SDS-PAGE)}

Protein separation according to their molecular weight was carried out by denaturing SDSPAGE. To generate polyacrylamide gel systems, resolving gel mixes were prepared (Table 12), poured between two glass plates with a spacing of either $1.5 \mathrm{~mm}$ or $0.75 \mathrm{~mm}$ set in a gel stand and overlaid with isopropanol. After polymerization at RT, the isopropanol was removed and the stacking gel was poured over the resolving gel. Immediately after pouring, a comb with 15 wells was inserted. The acrylamide concentration and gel thickness used depended on the expected mass of the protein of interest and the purpose of the experiment. For 
immunoblot applications, $1.5 \mathrm{~mm} 8 \%$ or $10 \%$ acrylamide gels were suitable. For in vitro kinase assays $0.75 \mathrm{~mm} 8 \%$ or $15 \%$ acrylamide gels were used. SDS-PAGE was carried out in the Mini-PROTEAN ${ }^{\circledR} 3$ system (BioRad, Munich, Germany). Before loading, the samples were boiled for $5 \mathrm{~min}$ at $95^{\circ} \mathrm{C}$ and centrifuged $1 \mathrm{~min}$ at full speed. Meanwhile, the gels were placed in the running apparatus and 1x SDS-running buffer was used to fill up the tank. Up to $20 \mu \mathrm{l}$ sample volume were loaded. PageRuler ${ }^{\mathrm{TM}}$ Prestained Plus protein Ladder (Thermo Fisher Scientific, Waltham, USA) was used as a size marker. $1.5 \mathrm{~mm}$ gels were run at $30 \mathrm{~mA} /$ gel and $0.75 \mathrm{~mm}$ gels were run at $100 \mathrm{~V}$ using a PowerPac ${ }^{\mathrm{TM}} \mathrm{HC}$ power supply (BioRad, Munich, Germany) until the bromophenol blue front reached the end. The gel apparatus was then disassembled and the gel was either stained directly with Coomassie brilliant blue (in vitro kinase assay) or used for Western blot experiments.

Table 12: Composition of mixtures used for resolving and stacking gel preparation in this study.

SDS PAGE Gel Buffers $(250 \mathrm{ml})$

$8 \%$ resolving gel buffer

$10 \%$ resolving gel buffer

$15 \%$ resolving gel buffer

Stacking gel buffer

\author{
$1 \mathrm{M}$ Tris- $\mathrm{HCl}(\mathrm{pH}=8.8)$ \\ $10 \% \mathrm{SDS}$ \\ $\mathrm{ddH}_{2} \mathrm{O}$
}
$1 \mathrm{M}$ Tris- $\mathrm{HCl}(\mathrm{pH}=8.8)$
$10 \%$ SDS
$\mathrm{ddH}_{2} \mathrm{O}$

$1 \mathrm{M}$ Tris- $\mathrm{HCl}(\mathrm{pH}=8.8)$

$10 \%$ SDS

$\mathrm{ddH}_{2} \mathrm{O}$

$1 \mathrm{M}$ Tris- $\mathrm{HCl}(\mathrm{pH}=6.8)$

$10 \%$ SDS

$\mathrm{ddH}_{2} \mathrm{O}$
$130.9 \mathrm{ml}$

$3.46 \mathrm{ml}$

$115.64 \mathrm{ml}$

$143.6 \mathrm{ml}$

$3.79 \mathrm{ml}$

$102.53 \mathrm{ml}$

$189.07 \mathrm{ml}$

$5.1 \mathrm{ml}$

$55.83 \mathrm{ml}$

$38.85 \mathrm{ml}$

$3.06 \mathrm{ml}$

$208.24 \mathrm{ml}$

\section{SDS-PAGE gel mixes $(10 \mathrm{ml})$}

\begin{tabular}{lll}
\hline $8 \%$ resolving gel & $8 \%$ resolving gel buffer & $7.2 \mathrm{ml}$ \\
& $30 \%$ acrylamide & $2.7 \mathrm{ml}$ \\
& APS $(10 \%)$ & $0.1 \mathrm{ml}$ \\
& TEMED & $0.006 \mathrm{ml}$ \\
$10 \%$ resolving gel & $10 \%$ resolving gel buffer & $6.6 \mathrm{ml}$ \\
& $30 \%$ acrylamide & $3.3 \mathrm{ml}$ \\
& APS $(10 \%)$ & $0.1 \mathrm{ml}$ \\
& TEMED & $0.004 \mathrm{ml}$ \\
\hline
\end{tabular}




\begin{tabular}{lll}
\hline $15 \%$ resolving gel & $15 \%$ resolving gel buffer & $4.9 \mathrm{ml}$ \\
& $30 \%$ acrylamide & $5.0 \mathrm{ml}$ \\
& APS (10\%) & $0.1 \mathrm{ml}$ \\
& TEMED & $0.004 \mathrm{ml}$ \\
Stacking gel & Stacking gel buffer & $8.16 \mathrm{ml}$ \\
& $30 \%$ acrylamide & $1.66 \mathrm{ml}$ \\
& APS (10\%) & $0.05 \mathrm{ml}$ \\
& TEMED & $0.005 \mathrm{ml}$ \\
\hline
\end{tabular}

\title{
2.2.4.5 Immunoblot analysis (Western blot)
}

Extracted proteins (2.2.5.1) were separated via SDS-PAGE (2.2.5.4) prior to immunoblotting. A PVDF membrane with a pore size of $0.45 \mu \mathrm{m}$ (Roth, Karlsruhe, Germany) was activated by dipping into methanol. Proteins were transferred onto the activated membrane by electro blotting in the TRANS-BLOT ${ }^{\circledR}$ CELL (BioRad, Munich, Germany) apparatus. The blotting apparatus was assembled as followed:

\author{
cathode \\ black grid of clamp \\ sponge \\ Whatman paper \\ gel (facing the cathode) \\ $\mathrm{MeOH}$ activated PVDF membrane \\ Whatman paper \\ sponge \\ transparent/red grid of clamp \\ anode
}

The blotting was performed in $1 \mathrm{x}$ transfer buffer at $100 \mathrm{~V}$ for $90 \mathrm{~min}$ at $4^{\circ} \mathrm{C}$. After disassembling the blotting apparatus, the PVDF membrane was blocked for at least $1 \mathrm{~h}$ with $10 \mathrm{ml}$ TBS-T $+4 \%$ milk powder at RT on a rotary shaker. After blocking, the primary antibody was added and the blots were incubated over night at $4{ }^{\circ} \mathrm{C}$ on a shaker.

The primary antibody was then removed and the membrane was washed 6 times with TBS-T $+4 \%$ milk powder for $15 \mathrm{~min}$. Next, the membrane was incubated in secondary antibody solution for $2 \mathrm{~h}$ at RT on a rotary shaker. After antibody incubation, the membrane was 
washed 6 times with 1x TBS-T for 15 minutes. Next, the membrane was equilibrated for at least 5 minutes in Alkaline Phosphatase (AP) buffer. $500 \mu 1$ Immun-Star ${ }^{\mathrm{TM}}$ AP substrate (BioRad, Munich, Germany), were equally distributed on the membrane in a plastic bag. To increase signal intensity, the membranes were incubated for $10 \mathrm{~min}$ in the dark. Afterwards the membranes were transferred to a new plastic bag and the chemiluminescence was detected using the ChemiDoc ${ }^{\mathrm{TM}}$ Touch detection device (BioRad, Munich, Germany).

\subsubsection{In vitro kinase assay}

For in vitro kinase assays, full length mCitrine tagged proteins were transiently expressed in N. benthamiana. Proteins were extracted (2.2.4.1.2) and a GFP-pull-down (2.2.4.3.2) was performed. Pull-downs were kept on ice at all times to prevent protein degradation or inactivation. Pull-downs were washed 3x with $15 \mathrm{ml}$ cold TBS-T with a final $\mathrm{NaCl}$ concentration of $1 \mathrm{M}$, afterwards beads were transferred to $1.5 \mathrm{ml}$ LoBind Eppendorf tubes. Beads were washed $3 \mathrm{x}$ in kinase buffer, dried with an insulin syringe and resuspended in $100 \mu 1$ kinase buffer. $20 \mu 1$ were transferred into a new tube and $40 \mu 1$ 1x SDS were added as Western Blot sample. The remaining $80 \mu \mathrm{l}$ beads were dried again and $16.8 \mu 1$ kinase assay mix were added to start the kinase reaction.

Kinase assay mix for one reaction:

$\begin{array}{ll}\text { Kinase buffer } & 15.0 \mu \mathrm{l} \\ \text { MBP }(10 \mathrm{mg} / \mathrm{ml}) & 1.5 \mu \mathrm{l} \\ \text { DTT }(100 \mu \mathrm{M}) & 0.1 \mu \mathrm{l} \\ {\left[\gamma-{ }^{32} \mathrm{P}\right]-\text { ATP }} & 0.2 \mu \mathrm{l}\end{array}$

$\left[\gamma^{32} \mathrm{P}\right]$-ATP with a specific activity of $111 \mathrm{~Bq} / \mathrm{mmol}$ and a concentration of $370 \mathrm{MBq} / \mathrm{ml}$ was used. The kinase reaction was incubated for 20-40 min at RT. $10 \mu 12$ x SDS loading dye were added to each sample to stop the reaction. Samples were boiled $2 \mathrm{~min}$ at $95^{\circ} \mathrm{C}$ and centrifuged 1 min at full speed. $5 \mu 1$ of the sample supernatant were loaded on an $8 \%$ and a $15 \%$ polyacrylamide gel and SDS-PAGE (2.2.5.4) was performed. Afterwards the gels were stained with Coomassie brilliant blue (2.2.5.7.1), dried (2.2.5.8) and exposed overnight to an Imaging Plate BAS-MP 2040S (Fujifilm Holdings Corporation, Tokyo, Japan). The imaging plate was developed using a Phosphoimager scanner FLA-3000 (Fujifilm, Tokyo, Japan) with the software BASREADER 3.14 (Fujifilm, Tokyo, Japan) and AIDA IMAGE data analyzer 3.24 (Elysia raytest GmbH, Staubenhardt, Germany). 


\subsubsection{Coomassie staining of SDS-PAGE gels and PVDF membranes}

\subsection{Staining of SDS-PAGE gels}

To visualize protein bands on an SDS gel, the gel was stained with Coomassie brilliant blue staining solution containing methanol. The gel was incubated in Coomassie staining solution for $5 \mathrm{~min}$ at room temperature while shaking. Afterwards, the gel was destained using destainer containing methanol. Depending on the staining intensity, destaining solution had to be changed once or several times. The whole experiment was performed under the fume hood.

\subsection{Staining of PVDF membranes}

PVDF membranes were stained with Coomassie brilliant blue containing ethanol to visualize protein bands. The PVDF membranes were covered with Coomassie staining solution and incubated for $5 \mathrm{~min}$ while shaking at RT. After incubation, the PVDF membrane was rinsed in water and the background was removed by incubating the membrane in destaining solution at RT while shaking. Finally, the membrane was rinsed in water again and dried.

\subsubsection{Drying of Coomassie stained SDS-PAGE gels}

Gels used for in vitro kinase assay had to be dried prior to autoradiography. Therefore, destained gels (2.2.4.7.1) were placed on Whatman paper, covered with cling film and dried in the PHERO-TEMP (BIOTEC-FISCHER GmbH, Reiskirchen, Germany) vacuum gel dryer at $80^{\circ} \mathrm{C}$ for $5 \mathrm{~h}$.

\subsubsection{Proteomics experiments}

\subsection{Sample preparation}

To identify phosphorylation sites in proteins, proteomics experiments were conducted. For this, proteins were extracted (2.2.4.1.2), and a GFP-pulldown was performed (2.2.4.3.2). After the pulldown, GFP-Trap agarose beads were washed three times with $10 \mathrm{ml} \mathrm{Wash/} \mathrm{Dilu-}$ tion buffer. To pellet the beads in between the washing steps, tubes were centrifuged $2500 \mathrm{~g}$ for $2 \mathrm{~min}$ at $4^{\circ} \mathrm{C}$ (Heraeus Fresco 17, Thermo Fisher Scientific, Waltham, USA). Washed beads were transferred to $1.5 \mathrm{ml}$ protein LoBind tubes (Eppendorf, Hamburg, Germany) and washed twice with $1 \mathrm{ml} \mathrm{Wash/} \mathrm{Dilution} \mathrm{buffer.} \mathrm{Afterwards} \mathrm{beads} \mathrm{were} \mathrm{dried} \mathrm{with} \mathrm{an} \mathrm{insulin}$ syringe and resuspended in 1x bead volume of Elution buffer I. Elution buffer I contains Sequencing Grade Modified Trypsin (Promega, Madison, Wisconsin, USA). The tubes were incubated in a thermomixer at $30^{\circ} \mathrm{C}$ and $400 \mathrm{rpm}$ for $90 \mathrm{~min}$ for an on-bead tryptic digestion. 
The supernatant was collected in a fresh vial. The beads were resuspended in $2 \mathrm{x}$ bead volume of elution buffer II and centrifuged for $2 \mathrm{~min}$ at $2500 \mathrm{~g}$ and $4^{\circ} \mathrm{C}$. The supernatant was combined with the supernatant from the previous step. The washing step with elution buffer II was repeated and the liquid was added to the supernatant pool. The tryptic digestion of the supernatant pool was continued overnight in a thermomixer at $32{ }^{\circ} \mathrm{C}$ and $400 \mathrm{rpm}$ in the dark. The next day $1 \mu 1$ trifluoroacetic acid (TFA) per $25 \mu$ l GFP-trap agarose bead volume was added to the samples to stop the reaction.

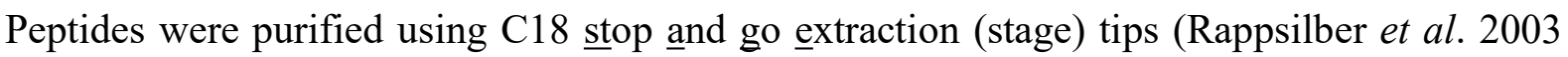
and 2007). For C18 purification, stage tips were prepared by washing with $30 \mu 1100 \%$ methanol in the first step, $30 \mu 1100 \%$ acetonitrile in the second step and $30 \mu 10.1 \%$ formic acid in the third step. To wash the C18 tips, they were placed with an adapter in $2 \mathrm{ml}$ Eppendorf tubes and centrifuged $2 \mathrm{~min}$ at $6000 \mathrm{~g}$ and $4^{\circ} \mathrm{C}$.

The prepared stage tips were placed with the adapter in a fresh $1.5 \mathrm{ml}$ protein LoBind tube and the samples were loaded to the tip. Air bubbles were removed by careful snipping the tips. To allow the sample to pass through the tips, the $\mathrm{C} 18$ tips placed in the $1.5 \mathrm{ml}$ tube were centrifuged $5 \mathrm{~min}$ at $1800 \mathrm{~g}$ and $4{ }^{\circ} \mathrm{C}$. Sample loading and centrifuging was repeated until all the sample was loaded on the tips. Afterwards the C18 tip was washed with $15 \mu 10.1 \%$ formic acid. The $\mathrm{C} 18$ tips were transferred into new $1.5 \mathrm{ml}$ Protein LoBind tubes and $50 \mu 1$ of 70 $\%$ acetonitrile $0.1 \%$ formic acid were added to elute the peptides by centrifuging for $4 \mathrm{~min}$ at $1800 \mathrm{~g}$ followed by a second step for $2 \mathrm{~min}$ at $3000 \mathrm{~g}$.

Purified peptides were vacuum dried at $45^{\circ} \mathrm{C}$ in the Eppendorf concentrator plus (Eppendorf, Hamburg, Germany). Dried peptide samples were dissolved in $20 \mu 1$ LC-MS sample buffer ( $2 \%$ acetonitrile, $0.1 \%$ formic acid).

\subsubsection{Mass spectrometry}

Mass spectrometry was performed at the Service Unit LCMS Protein Analytics at the Institute of Microbiology and Genetics, Georg August-Universtity Göttingen.

$2 \mu \mathrm{l}$ of each sample were subjected to reverse phase liquid chromatography for peptide separation using an RSLCnano Ultimate 3000 system (Thermo Fisher Scientific, Waltham, Massachusetts, USA). Peptides were loaded on an Acclaim ${ }^{\circledR}$ PepMap 100 pre-column (100 $\mu \mathrm{m} \mathrm{x}$ $2 \mathrm{~cm}, \mathrm{C} 18,5 \mu \mathrm{m}, 100 \AA$; Thermo Fisher Scientific) with $0.07 \%$ trifluoroacetic acid. Analytical separation of peptides was done on an Acclaim ${ }^{\circledR}$ PepMap RSLC column $(75 \mu \mathrm{m} \times 50 \mathrm{~cm}$, C18, $2 \mu \mathrm{m}, 100 \AA \AA$; Thermo Fisher Scientific) running a water-acetonitrile gradient at a flow rate of $300 \mathrm{nl} / \mathrm{min}$. All solvents and acids had Optima grade for LC-MS (Thermo Fisher Sci- 
entific). Chromatographically eluting peptides were on-line ionized by nano-electrospray (nESI) using the Nanospray Flex Ion Source (Thermo Scientific) at $1.5 \mathrm{kV}$ (liquid junction) and continuously transferred into the mass spectrometer ( $Q$ Exactive $H F$, Thermo Scientific). Full scans in a mass range of 300 to $1650 \mathrm{~m} / \mathrm{z}$ were recorded at a resolution of 30,000 followed by data-dependent top 10 fragmentation (HCD) at a resolution of 15,000 (dynamic exclusion enabled) (Kerstin Schmitt, personal communication).

For targeted single-ion monitoring (tSIM) LC-MS runs, the resolution was set to 60,000. The maximum ion time for tSIM scans (AGC target 1e6) was $100 \mathrm{~ms}$. The loop count equalled the number of $\mathrm{m} / \mathrm{z}$ values on the inclusion list. Dynamic exclusion was disabled. LC-MS method programming and data acquisition was performed with the XCalibur software (4.0, Thermo Fisher Scientific, Waltham, Massachusetts, USA).

For proteins extracted from $N$. benthamiana, database searches were performed against a $N$. benthamiana protein database (Kourelis et al., 2019) and the CRK7 as well as CRK7-4 pro-

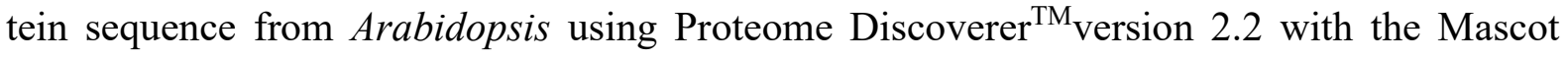
and SequestHT search algorithms. For proteins extracted from Arabidopsis, the database searches were performed against the Araport11 protein database (Cheng et al., 2017). For both, the digestion mode was set to trypsin and the maximum of missed cleavage sites to three. Carbamidomethylation of cysteines was set as fixed modification, oxidation of methionines and phosphorylation were set as variable modifications. The mass tolerance was 10 ppm for precursor ions and 0.6 Da for fragment ions. The decoy mode was reverse with a false discovery rate of 0.01 (Kerstin Schmitt, personal communication).

\subsubsection{Bioinformatics}

2.2.5.1 Mapping of Next Generation Sequencing (NGS) data to the Arabidopsis reference genome.

CLC genomic workbench 10.1.1 (Qiagen, Hilden, Germany) was used to map either $50 \mathrm{bp}$ non-paired or 150 bp paired-end Illumina sequencing reads to the Arabidopsis TAIR10 reference genome.

The sequencing data were imported and the standard parameters used for aligning were a length fraction of 0.85 and a similarity fraction of 0.8 . This means that $85 \%$ of the alignment must match the reference sequence before the read is included in the mapping. Within the matching part of the sequence a maximum of $20 \%$ mismatches is allowed. Broken read-pairs were ignored. SNPs were identified with the basic variant detection tool. The standard pa- 
rameters for this tool were a minimum coverage of 10 , a minimum count of the identified SNP of two and a minimum SNP frequency of $20 \%$. The base quality for the SNP itself was set to 20 and the base quality filter for the five neighboring base pairs was set to 15 . The generated table was exported and further analyzed using Excel (Microsoft Corporation, Albuquerque, New Mexico, USA).

\subsubsection{2 guide RNA design}

To design guide RNAs for CRISPR/ Cas9, four different online tools were used. The sequences $500 \mathrm{bp}$ upstream of the ATG and $500 \mathrm{bp}$ downstream of the TGA of the gene of interest were used as queries. The used tools were E-CRISP (Heigwer et al., 2014), CRISPRdirect (Naito et al., 2015), CRISPOR (Haeussler et al., 2016) and CRISPR-P v2.0 (Liu et $a l ., 2017 \mathrm{a})$. The three best hits of each of these different online tools were compared and the three most frequently suggested guide RNAs among the different programs for the 5 'prime and 3'prime region were chosen. The three 5'guide RNAs were combined with the three 3' prime guide RNAs in all different combinations resulting in nine plasmids. 


\section{Results}

\subsection{CRK7}

3.1.1 Mapping by sequencing revealed a single amino acid exchange in CRK7 as possible suppressor mutation

The cerk1-4 mutant develops an increased salicylic acid (SA) dependent cell death response upon infection with powdery mildews, resulting in enhanced resistance. Similar cell death responses were observed in uninfected cerk1-4 plants older than five weeks (Petutschnig et al., 2014). Moreover, it was previously shown that the CERK1 receptor undergoes ectodomain shedding, resulting in the release of a soluble ectomain fragment which is detectable in Western Blot analysis. Notably, this ectodomain fragment is missing in cerkl-4 plants (Petutschnig et al., 2014). Despite these interesting observations, the molecular mechanisms underlying aberrant SA accumulation, absence of shed ectodomains and deregulated cell death execution, as well as their potential interrelations, remained unclear.

Thus, a forward genetic suppressor screen was started to identify molecular components required for these cerkl-4 associated phenotypes. In this screen, a population of EMS mutagenized cerk1-4 seeds was harvested in 306 batches of about 60 plants per batch (M. Stolze, unpublished). In the M2 generation, plants were scored with regard to cell death patterns and fungal growth upon E. cruciferarum infection. Plants without cell death but with fungal growth were considered as suppressors.

For this work, seeds of batch nine were infected with E. cruciferarum in the M2 generation, which yielded 26 plants showing a cerkl-4 suppressor phenotype (E. Petutschnig, unpublished). Suppressor plants were named noce 9 (no-cerk1-4 cell death phenotype batch 9).

Ten of these identified suppressors were inoculated with E. cruciferarum again in the M3 generation and analyzed for fungal growth and cell death. Lines in which the suppressor phenotype was confirmed were back-crossed to cerkl-4 to allow the mutations to segregate (E. Petutschnig and J. Anders). noce9-1, a suppressor line with no cell death and a very strong fungal growth was chosen for further analysis in this work.

For mapping, 218 three-week old noce9-1 x cerk1-4 F2 plants were infected with E. cruciferarum. Col-3 gll and cerkl-4 served as control plants and were randomly distributed among the F2 plants on the trays prior to infection. Infected plants were scored at different time points. The final scoring was made four weeks post the first infection as it turned out that the phenotype became clearer with time. 45 of the 218 infected plants (20.6 \%) showed a clear suppressor phenotype with strong fungal growth on the leaf surface and no cell death. 42 F2 
plants (19.3\%) showed a clear cerk1-4 cell death phenotype. Surprisingly most of the plants, 131 of $218(60.1 \%)$, showed an intermediate phenotype with only mild cell death and no or just very low amount of fungal growth. Example pictures of plants with suppressor, nonsuppressor and intermediate phenotypes are shown in figure 4 . The fact that not only suppressor and non-suppressor plants but also plants with an intermediate phenotype were observed, suggests that the mutation causing the suppressor phenotype in noce $9-1$ is semi-dominant.

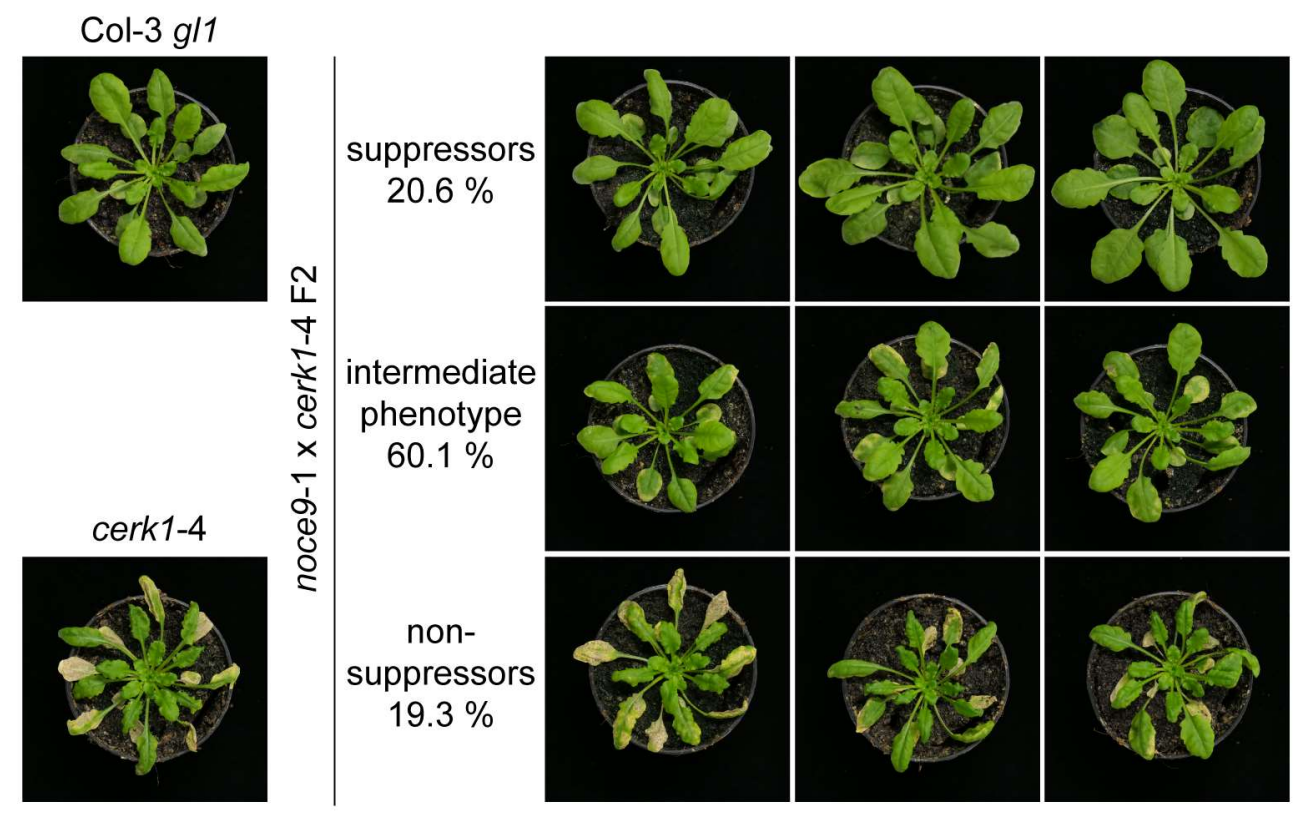

Figure 4: $E$. cruciferarum infected noce9-1 x cerk1-4 F2 plants show a semi-dominant suppressor phenotype.

Four-week old noce9-1 x cerk1-4 F2 plants and the controls Col-3 gll and cerkl-4 were infected with E. cruciferarum. Pictures were taken 11 days post infection (dpi). A total number of 218 plants was scored according to cell death and fungal growth. Plants showing no cell death and strong fungal growth were considered as suppressors, plants with no fungal growth but strong cell death such as in the cerkl-4 control were classified as non-suppressors and the remaining plants with no or a very low amount of fungal growth and mild cell death were classified as intermediate phenotype. Numbers below the categories show the percentage of plants with the according phenotype based on 218 analyzed plants.

Leaf material from the suppressor plants with the clearest and strongest suppressor phenotypes $(\mathrm{N}=34)$ was harvested and pooled and genomic DNA was extracted from this pool (2.2.3.1.2). The genomic DNA was used for next generation sequencing. 50 bp non-paired Illumina sequencing and $150 \mathrm{bp}$ paired-end Illumina sequencing reads were generated to achieve at least 20-fold coverage of the genome. The sequencing results of both non-paired and paired reads, 173 million reads in total, were aligned to the Arabidopsis TAIR 10 reference genome using CLC genomics workbench 10.1.1. SNPs in noce9-1 compared to the TAIR 10 reference genome were identified (2.2.5.1). 
The cerk1-4 mutant itself was also identified after an EMS mutagenesis in a forward genetic suppressor screen (Petutschnig et al., 2014). Since noce9-1 is a mutant based on cerk1-4, most of the SNPs from the mutagenesis of the first screen are also present in the genome of noce9-1. Therefore, SNPs that occurred in both cerk1-4 and noce9-1 were removed. Sequencing data for three more suppressor mutants from independent batches were generated either before (noce1-4; M. Stolze, C. Meusel, E. Petutschnig, unpublished) or during this work (noce8-1, noce12-23). SNPs identified in noce9-1 were compared to the sequencing data from these three lines and duplicates were removed in order to obtain noce9-1 specific SNPs. The SNP frequencies of all remaining SNPs present in noce9-1 were plotted against their chromosomal position (Figure 5). On all chromosomes one area with an elevated SNP number and frequency is visible. This area matches the position of the centromere. The centromere region consists of large arrays of repetitive DNA elements with highly similar but not identical sequences (Round et al., 1997; Copenhaver et al., 1999), causing problems for the alignment program. Therefore, the majority of these SNPs can be considered as artefacts due to the alignment process. SNPs that are not linked to the suppressor mutation are expected to show a SNP frequency of about $50 \%$ in the suppressor pool. The suppressor mutation itself should have a SNP frequency of $100 \%$ as only plants with a suppressor phenotype were used for sequencing. Mutations that are physically near to the suppressor mutation are genetically linked and this linkage is stronger, the closer a random mutation is located to the suppressor mutation. Therefore, genetically linked SNPs have expected SNP frequencies higher than 50 $\%$, increasing with proximity to the causative mutation. Ideally this results in a peak of the SNP frequency. For noce9-1 such a peak is clearly visible on the lower arm of chromosome four (Figure 5), suggesting the suppressor mutation to be encoded in this area.

SNPs with a $100 \%$ SNP frequency on top of the peak are the best candidates for the suppressor mutation (Table 13). SNPs in intergenic areas are not included in the table. The expression of the candidates in table 13 was investigated using RNA sequencing data (E. Petutschnig, unpublished; Table 13). These data revealed that the expression of only one of the candidate genes, cysteine-rich receptor-like protein kinase 7 (CRK7, At4g23150), was highly induced in Col-3 gll plants upon infection with the powdery mildew fungi Blumeria graminis f.sp. hordei (Bgh) compared to uninfected Col-3 gll plants. Interestingly, this induction is even higher in Bgh infected cerkl-4 plants compared to uninfected cerkl-4 plants. Consequently, the best candidate for the suppressor mutation is the mutation identified in CRK7. 

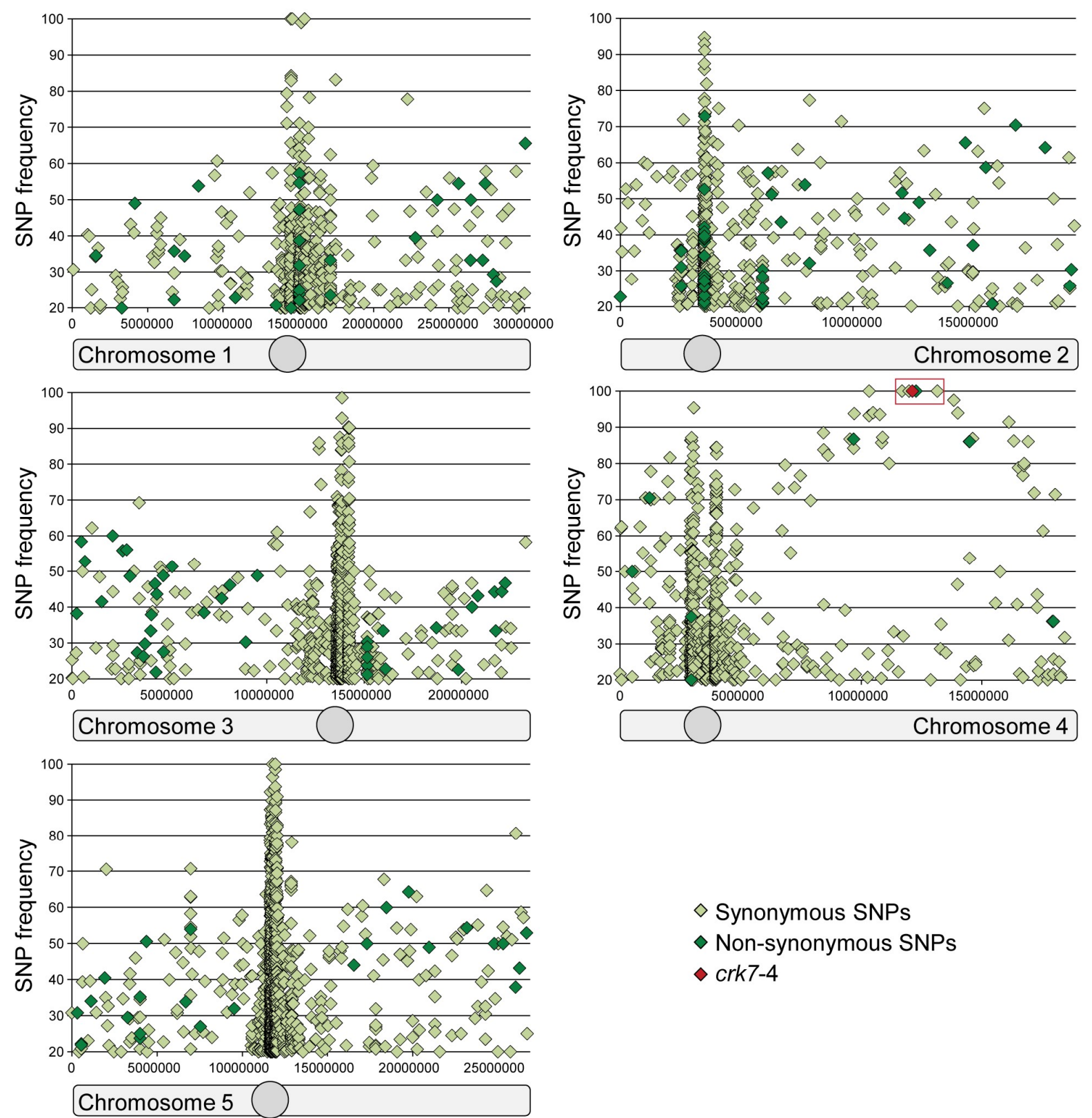

$\diamond$ Synonymous SNPs

$\diamond$ Non-synonymous SNPs

$\diamond \mathrm{crk} 7-4$

Figure 5: The suppressor mutation in noce9-1 is located on the lower arm of chromosome four. noce9-1 suppressor SNP frequencies were mapped to their position on the chromosomes. 50 bp unpaired and $150 \mathrm{bp}$ paired end Illumina reads were combined and aligned to the TAIR 10 reference genome using CLC genomics workbench 10.1.1. Parameter for aligning and SNP call: Length fraction: 0.9; Similarity fraction: 0.9 ; minimum coverage $=20$, minimum counts $=2$, minimum frequency $=20 \%$; minimum central quality $=20$; minimum neighborhood quality= 15. SNPs that were present also in cerk1-4, noce1-4, noce8-1 and noce12-23 were subtracted. Synonymous SNPs are shown as light green diamonds, SNPs causing non-synonymous mutations are depicted as dark green diamonds. Grey boxes below the graphs symbolize chromosomes with circles marking the centromeres. The red box highlights the region containing the suppressor mutation. The best candidate due to analysis of expression data, crk7-4, is depicted as a red diamond. 
Table 13: Suppressor candidates in noce9-1.

\begin{tabular}{|c|c|c|c|c|c|c|c|}
\hline \multirow{2}{*}{$\begin{array}{l}\text { Reference } \\
\text { position }\end{array}$} & \multirow[t]{2}{*}{ SNP } & \multirow{2}{*}{$\begin{array}{l}\text { Overlapping } \\
\text { annotations }\end{array}$} & \multirow{2}{*}{$\begin{array}{l}\text { Coding } \\
\text { region } \\
\text { change }\end{array}$} & \multirow{2}{*}{$\begin{array}{l}\text { Amino acid } \\
\text { change }\end{array}$} & \multirow[t]{2}{*}{ Annotation $^{\mathrm{a}}$} & \multicolumn{2}{|c|}{$\begin{array}{l}\text { Bgh induced fold- } \\
\text { induction }^{\mathrm{b}}\end{array}$} \\
\hline & & & & & & Col-3 gll & cerk1-4 \\
\hline 11995118 & $\mathrm{G}>\mathrm{A}$ & At4g22850 & $\begin{array}{l}489+106 \\
G>A\end{array}$ & & $\begin{array}{l}\text { SNARE associated } \\
\text { Golgi protein family }\end{array}$ & 0.90 & 0.94 \\
\hline 12126011 & $\mathrm{G}>\mathrm{A}$ & $\begin{array}{l}\text { At4g23150 } \\
\text { CRK7 }\end{array}$ & $281 \mathrm{G}>\mathrm{A}$ & Cys94Tyr & $\begin{array}{l}\text { cysteine-rich receptor-like } \\
\text { protein kinase }\end{array}$ & 14.73 & 30.59 \\
\hline 12279873 & $\mathrm{G}>\mathrm{A}$ & $\begin{array}{l}\text { At4g23530 } \\
\text { ROH1 }\end{array}$ & $703 \mathrm{G}>\mathrm{A}$ & Val235Met & putative DUF793 protein & 1.06 & 1.35 \\
\hline 13163022 & $\mathrm{G}>\mathrm{A}$ & At4g25900 & $691 \mathrm{G}>\mathrm{A}$ & Asp231Asn & $\begin{array}{l}\text { Galactose mutarotase-like } \\
\text { superfamily protein }\end{array}$ & 1.43 & 2.10 \\
\hline
\end{tabular}

a www.arabidopsis.org

${ }^{b}$ E. Petutschnig, unpublished data. Similar results obtained in three independent replicates.

Cysteine-rich receptor-like protein kinases are one of the largest subgroups among Arabidopsis RLKs and have a highly conserved domain architecture with two DUF26 domains in the ectodomain. DUF26 domains contain a highly conserved motif with three conserved cysteine residues $\left(\mathrm{C}-\mathrm{X}_{8}-\mathrm{C}-\mathrm{X}_{2}-\mathrm{C}\right)$ (Figure 6; Figure $\mathrm{S} 1$ ). These cysteine residues were shown to be important for structure and stability of DUF26 domains and the function of DUF26 proteins (Lee et al., 2017; Yadeta et al., 2017; Vaattovaara et al., 2019).

The SNP identified in CRK7 in noce9-1 is an exchange of guanine ${ }^{281}$ to alanine causing a single amino acid exchange of the second cysteine in the first DUF26 domain (cys $\left.{ }^{94}\right)$ to tyrosine. This mutation was named crk7-4 (Figure 6), since three crk7 T-DNA lines were already described in a previous study (Bourdais et al., 2015).

Genotyping of single suppressor and non-suppressor plants revealed that all 34 suppressors used for mapping were homozygous for the $c r k 7$ mutation whereas the non-suppressors were WT for $C R K 7$, confirming the mapping results (Figure S2A). Subsequently, CRK7 was genotyped in all 26 suppressor mutants isolated from batch 9. The crk7 mutation was present in 17 lines, indicating that they all derived from the same M1 mother plant (Figure S2B). 
A
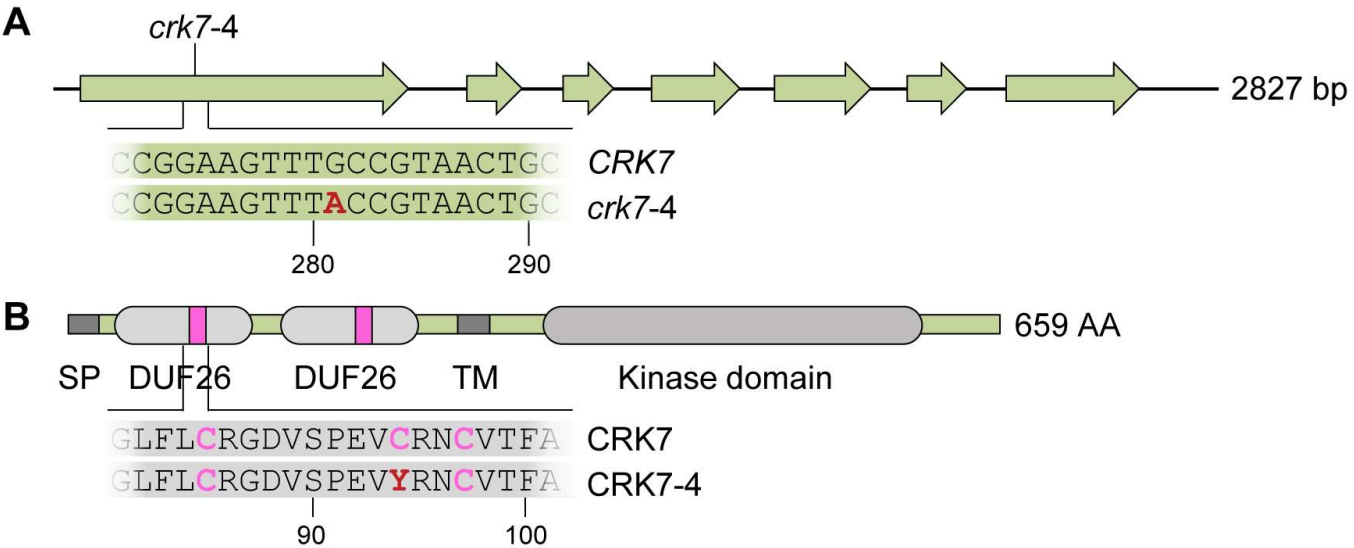

Figure 6: The crk7-4 mutation identified in the suppressor screen.

A: Schematic structure of the $C R K 7$ gene; exons are depicted as light green arrows. The DNA sequence alignment shows the guanine ${ }^{281}$ to adenine nucleotide exchange (red) in crk7-4. B: Schematic structure of the CRK7 protein. Predicted protein features: Signal peptide (SP), DUF26 domains with the internal C$\mathrm{X}_{8}-\mathrm{C}-\mathrm{X}_{2}-\mathrm{C}$ motif (pink box), transmembrane domain (TM) and kinase domain. Domains were predicted using SMART (http://smart.embl-heidelberg.de/, Letunic and Bork, 2018). The protein sequence alignment shows the cysteine ${ }^{94}$ to tyrosine amino acid exchange in crk7-4 (red). Highly conserved cysteine residues are depicted in pink.

The increased cell death response in cerkl-4 plants is salicylic acid (SA) dependent and requires SA synthesis and signaling components. cerk1-4 plants accumulate SA to a considerably higher level than WT plants upon Bgh infection or in uninfected senescent plants (Petutschnig et al., 2014). In cells with high SA levels, transcription of Pathogenesis Related (PR) genes is upregulated (Malamy et al., 1990). Accordingly, expression of the SA marker gene $P R I$ was shown to be significantly higher in $B g h$ infected cerkl-4 plants than in infected WT plants (Petutschnig et al., 2014). Therefore, PRl expression levels in cerkl-4 crk7-4 and crk7-4 plants were investigated (Figure 7). The crk7-4 single mutant, obtained from a cross of cerk1-4 crk7-4 with Col-3 gll, was included in the study to investigate if this mutation causes a cerk1-4 independent phenotype. 4.5-week-old Col-3 gll, cerk1-4, cerk1-4 crk7-4 and crk74 plants were infected with Bgh or Erysiphe cruciferarum. Uninfected control plants for every line where grown under short day conditions. Seven days later whole rosettes of six uninfected, Bgh infected and E. cruciferarum infected plants per line were photographed and harvested (Figure 7A). Rosettes of another six E. cruciferarum infected plants per line were photographed and harvested 14 dpi (Figure 7A). 

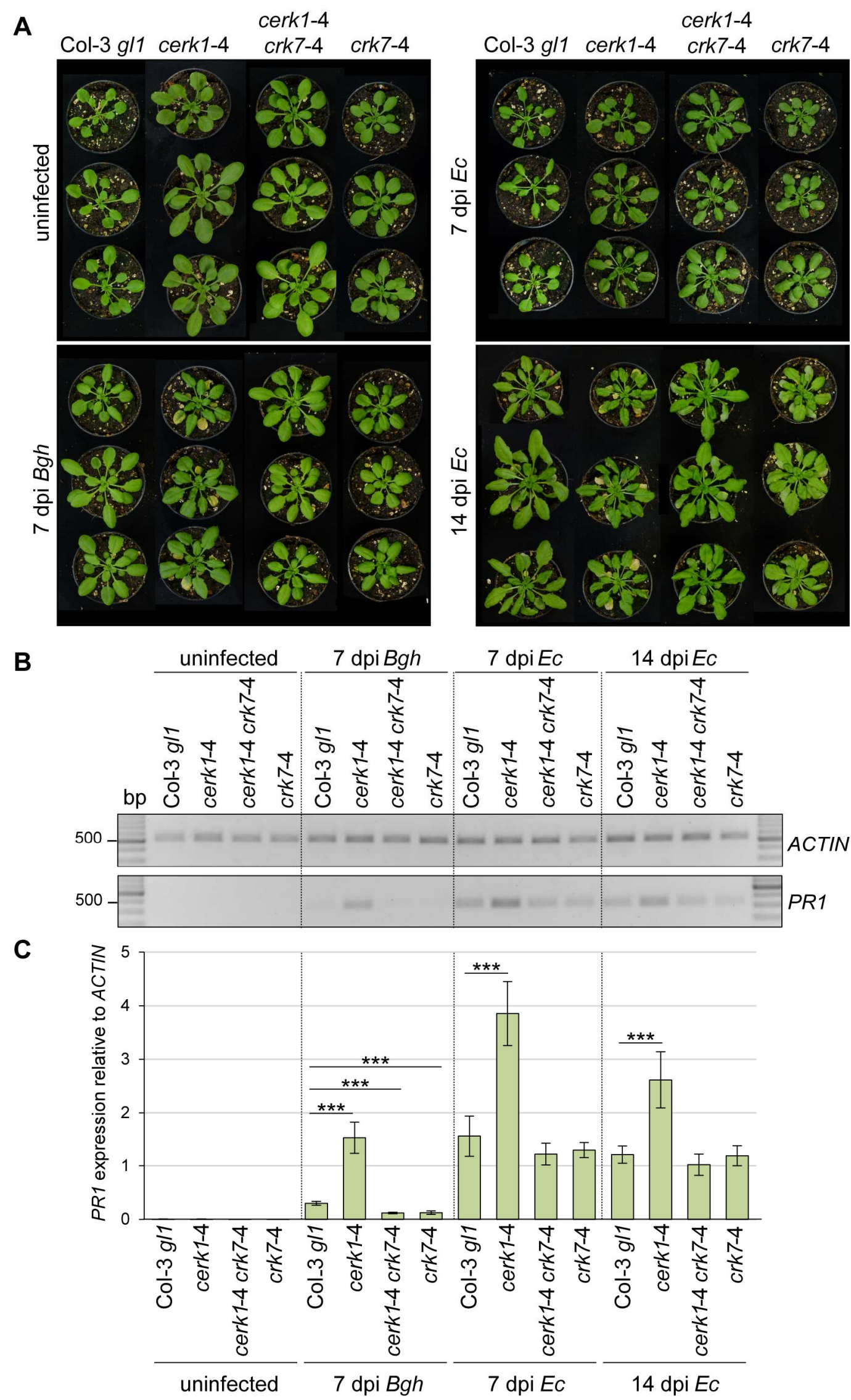
Figure 7: A mutation in $C R K 7$ suppresses increased powdery mildew induced PR1 gene expression in cerk1-4.

4.5 week-old plants were either infected with Blumeria graminis f.sp. hordei (Bgh), Erysiphe crucifer$\operatorname{arum}(E c)$ or kept uninfected. Whole rosettes of six infected plants per line were harvested either 7 dpi (Bgh and $E c$ ) or 14 dpi $(E c)$. RT-PCR was performed on PRl with $A C T I N$ as a control. A: Representative composite images of uninfected and infected plants. B: Semi-quantitative RT-PCR. This experiment was conducted in three independent biological replicates with similar results. C: Quantitative real-time PCR. This experiment was conducted for one biological replicate from B. The data show averages of four technical replicates. Error bars represent StDev. A student's t-test was made for statistical analysis, ${ }^{* * *} \mathrm{p} \leq$ 0.005 .

PRI and ACTIN expression were investigated via semi-quantitative (2.2.3.15; Figure 7B) and quantitative RT-PCR (2.2.3.16; Figure 7C). In uninfected plants, PRI expression was below detection level (Figure 7B). As described before (Petutschnig et al., 2014), PR1 expression in Bgh infected cerkl-4 plants was higher than in infected WT plants. The same was observed for both time points after E. cruciferarum infection (Figure 7B). cerk1-4 crk7-4 and crk7-4 plants showed PRI expression levels similar to WT after both Bgh and E. cruciferarum infection (Figure 7B).

qRT-PCR data showed that the overall PRI expression is higher in E. cruciferarum infected plants than in Bgh infected plants. E. cruciferarum infected cerk1-4 crk7-4 and crk7-4 plants express $P R 1$ at a WT-like level whereas $P R 1$ expression in cerk1-4 is significantly higher compared to WT. Upon $B g h$ inoculation, $P R l$ expression was even slightly lower in cerkl-4 crk7-4 and crk7-4 plants than in WT (Figure 7C).

The fact that the high $P R 1$ expression level observed in infected cerk1-4 plants is reversed to WT levels in the cerk1-4 crk7-4 double mutant suggests that CRK7 is necessary to induce high SA levels in cerk1-4 plants. Consequently, the SA dependent cerk1-4 cell death does not occur in crk7 plants.

\section{$\underline{\text { 3.1.2 Confirmation of CRK7 as a molecular component of cerk1-4 cell death induction }}$}

To confirm $c r k 7-4$ as the suppressor mutation in noce9-1, independent $c r k 7$ mutant lines were ordered (3.1.2.1), additionally identified in the screen (3.1.2.2) or generated in this work (3.1.2.3). All lines were analyzed towards their ability to suppress the cerkl-4 cell death response. 
3.1.2.1 Analysis of CRK7 T-DNA insertion lines

\subsection{Isolation of the $c r k 7-5$ T-DNA insertion line}

The T-DNA insertion line GK643A06 was ordered, a T-DNA insertion in the first exon of CRK7 could be verified (Figure 8A and C) and homozygous plants could be isolated. The exact position of the T-DNA insertion was determined by PCR and sequencing (Figure 8A and C). These analyses also revealed that GK643A06 harbors an inverted tandem repeat insertion with two left T-DNA borders and a 59 bp deletion in exon one (Figure 8A). This mutant was named crk7-5. The T-DNA insertion in $c r k 7-5$ disrupts the gene in the region encoding the first DUF26 domain (Figure 8B).

Previously described T-DNA lines (Bourdais et al., 2015) were either no longer available from NASC (crk7-1, WiscDSLox434B3) or did not harbor the predicted T-DNA insertion (crk7-3, SALK151109). For crk7-2 (WiscDsLox502A10) the T-DNA insertion could be verified, but homozygous plants could not be obtained despite multiple attempts in two different generations.

To investigate if $C R K 7$ expression is altered in $c r k 7-5$, semi-quantitative RT-PCR was performed with E. cruciferarum infected crk7-5 and control plants, using the primers indicated in figure 8A. CRK7 transcript was never detected in $c r k 7-5$ with the oligonucleotides oCT26 and $\mathrm{oCT} 27$, that are spanning the T-DNA insertion (Figure 8D). Nevertheless, a reduced amount of $C R K 7$ transcript was detected with oCT49 and oCT36, that are both binding to CRK7 downstream of the T-DNA insertion (Figure 8D). This is most likely due to the fact that a promotor is present in the vector near the left border of the inserted T-DNA (Ülker et al., 2008).

The same $C R K 7$ expression results were also obtained in cerk1-4 crk7-5 double mutant plants (Figure 8D), which were generated for suppression studies by crossing cerkl-4 and crk7-5 (see 3.1.2.1.2). 
A

crk7-5

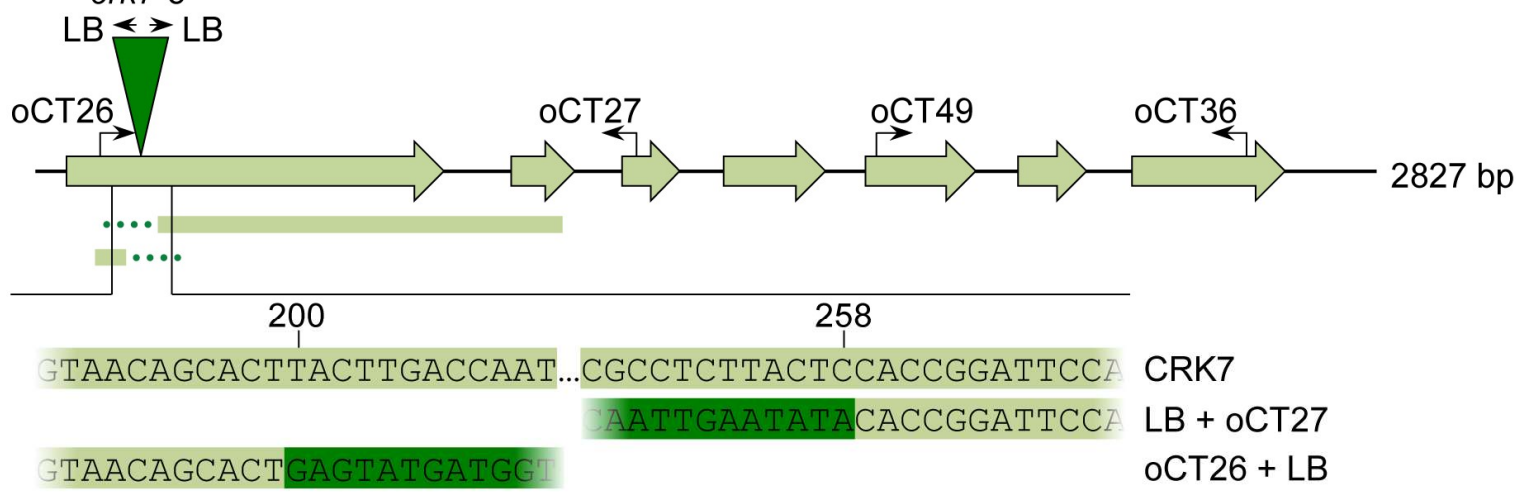

B

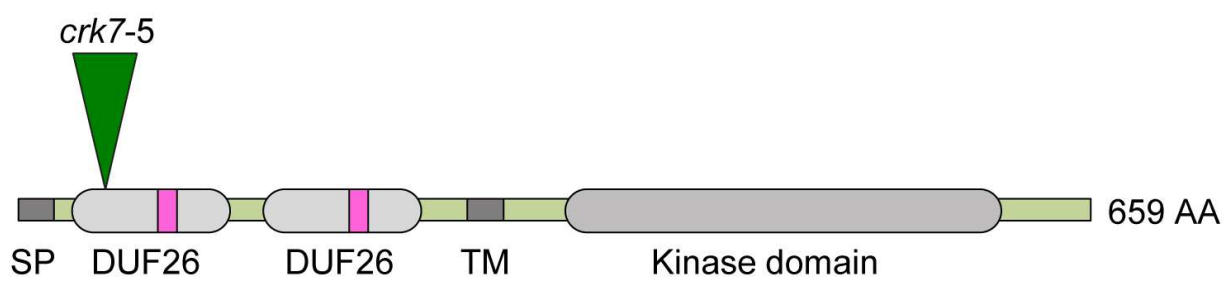

C

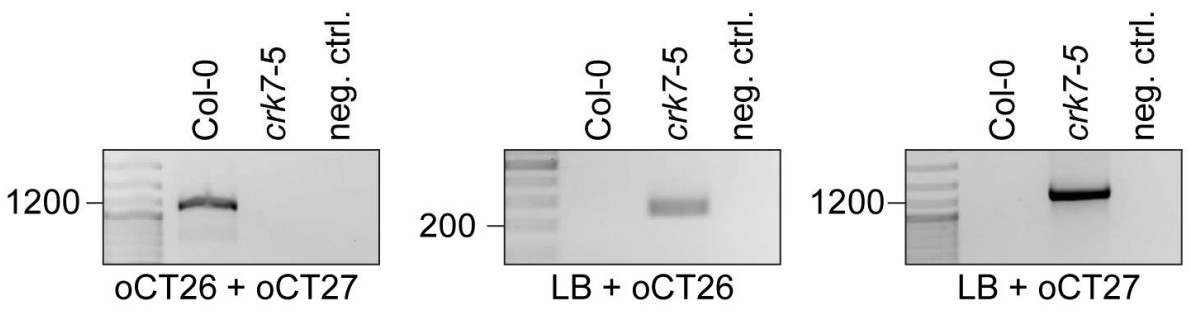

D

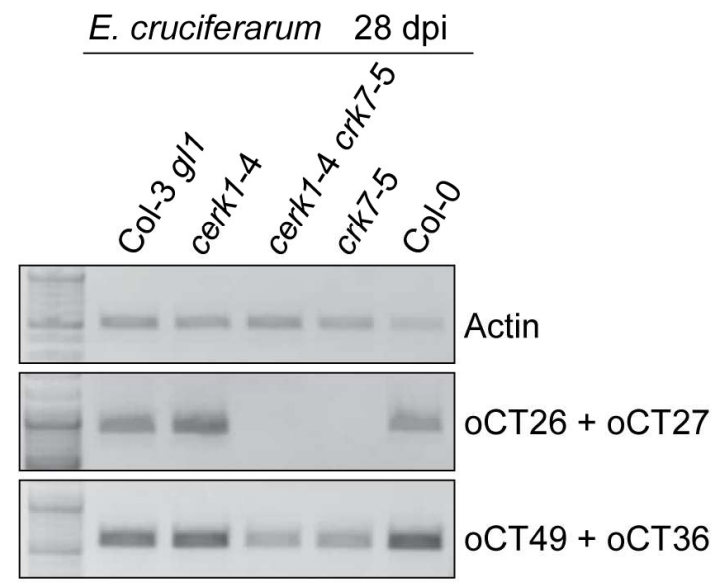

Figure 8: Characterization of the $c r k 7-5$ T-DNA insertion line used in this study.

A: Schematic structure of the CRK7 gene. Exons are depicted as light green arrows. Primers used for genotyping are indicated with black arrows. The T-DNA insertion is depicted as a green triangle; $\mathrm{LB}=$ Left border. PCR products shown in $\mathrm{C}$ were sequenced and a section of the sequencing results is shown. Light green areas align to WT $C R K 7$, dark green areas align to the T-DNA. B: Schematic structure of the CRK7 protein. Predicted protein features: Signal peptide (SP), DUF26 domains with the internal C- $\mathrm{X}_{8}-\mathrm{C}-$ $\mathrm{X}_{2}-\mathrm{C}$ motif (pink box), transmembrane domain (TM) and kinase domain. Domains were predicted using SMART (http://smart.embl-heidelberg.de/, Letunic and Bork, 2018). The position of the T-DNA insertion is shown as a green triangle in A and B. C: PCR-based genotyping of crk7-5. Homozygosity was verified using the primers indicated in A. PCR products were sequenced and sequencing results are shown in A. D: Semi-quantitative reverse transcription PCR with the indicated primers was performed on RNA extracted from pools of five E. cruciferarum infected plants per line. dpi = days post infection. 
3.1.2.1.2 The $c r k 7-5$ T-DNA insertion suppresses the cerkl-4 cell death phenotype

A population of plants homozygous for cerk1-4 but segregating for crk7-5, derived from a cross of cerk1-4 with crk7-5, was infected with E. cruciferarum together with a line homozygous for cerk1-4 and segregating for crk7-4. The control plants Col-3 gll, cerk1-4, crk7-4, Col-0 and crk7-5 were included in the experiment. All segregating plants were genotyped for crk7-4 and crk7-5 respectively. Four-week old plants were randomized and used for infection (2.2.1.1.4.2). The plants were scored according to cell death formation as well as fungal growth and pictures of the infected plants were taken nine dpi.

As expected, plants without a mutation in CRK7 showed cerk1-4 cell death (Figure 9). Plants heterozygous for crk7-4 showed the intermediate phenotype as observed in the F2 mapping population of the screen (3.1.1) with mild cell death and a low amount of fungal growth. Homozygous cerk1-4 crk7-4 plants showed a full suppressor phenotype. The same holds true for homozygous cerk1-4 crk7-5 plants, confirming the EMS-induced single amino acid exchange of cysteine ${ }^{94}$ to tyrosine in CRK7 as the suppressor mutation in noce9-1. Moreover, these results confirm $c r k 7-5$ as a $C R K 7$ knockout mutant. Interestingly, plants homozygous for cerk 1-4 and heterozygous for crk7-5 also showed the intermediate phenotype. Amino acid exchanges in proteins can lead to reduced function or even gain of function variants and can therefore cause semi-dominant or dominant phenotypes. For T-DNA insertion lines a semidominant phenotype is quite unusual and suggests either some activity of a potentially existing truncated protein or a dose-dependency of CRK7 transcript or protein level in cerk1-4 cell death induction. 

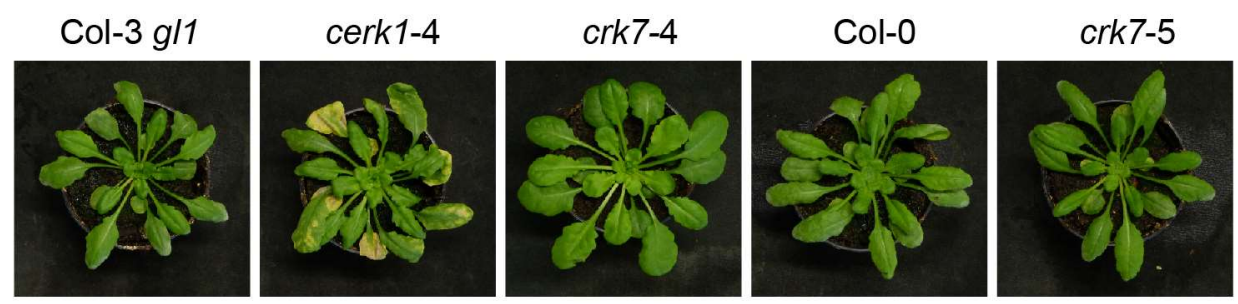

cerk1-4 / cerk1-4
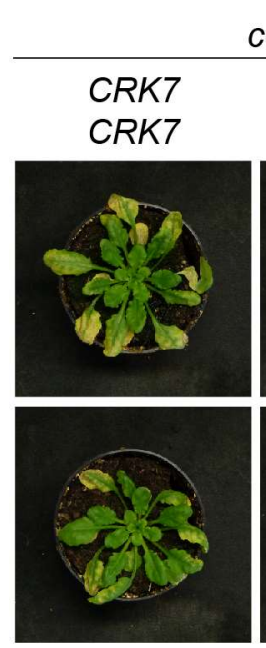

\begin{abstract}
CRK7
\end{abstract}
crk7-4
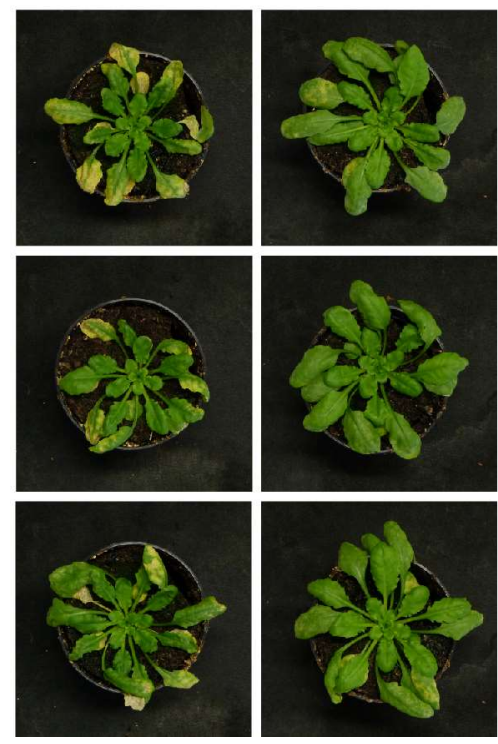

crk7-4

crk7-4
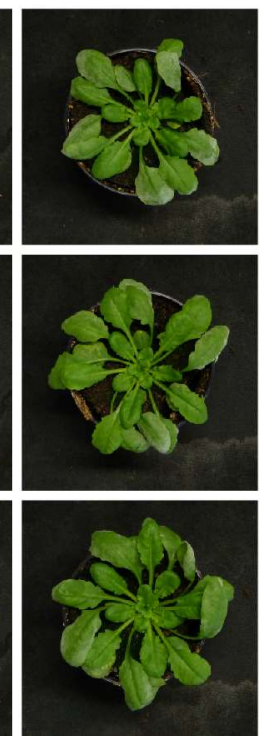
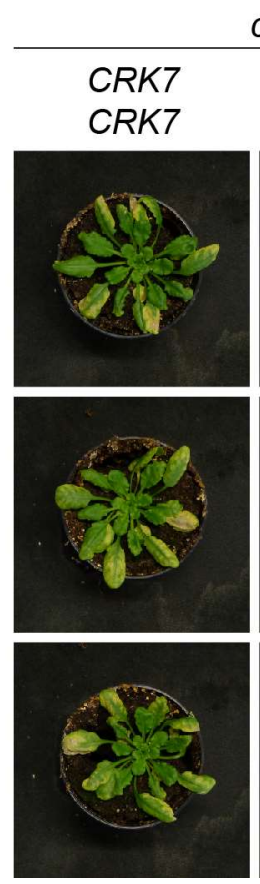

cerk1-4 / cerk1-4

CRK7 crk7-5

crk7-5
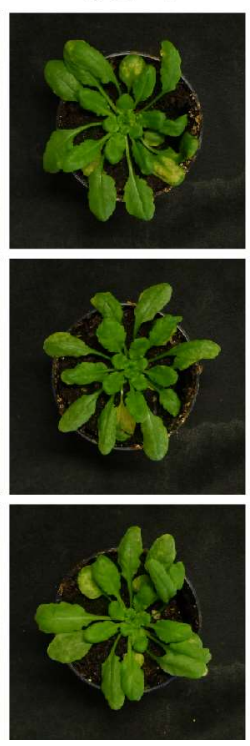

crk7-5
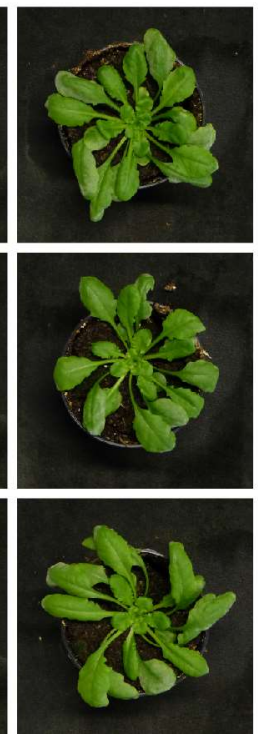

Figure 9: cerk1-4 crk7-4 and cerk1-4 crk7-5 show semi-dominant phenotypes upon $E$. cruciferarum infection.

Four-week-old plants were randomized and infected with E. cruciferarum. Pictures of infected plants were taken nine days post infection and images of three representative plants are shown per genotype. Genotypes are indicated above the plant pictures.

\subsubsection{An independent $C R K 7$ mutant isolated in the cerk1-4 suppressor screen}

To identify additional components required for cerkl-4 cell death induction, the screen was continued by analyzing additional mutant lines (C. Trippel, L. Pierdzig, E. Petutschnig, A. Vasquez). One line, noce13-34, was segregating in the M3 generation in a semi-dominant pattern similar to noce9-1. CRK7 was sequenced in four noce13-34 plants fully suppressing the cerk $1-4$ cell death phenotype. All of them carried the same mutation, a single nucleotide exchange of the last base pair of intron six, guanine ${ }^{2314}$ to adenine. This mutation was named crk7-6 (Figure 10A).

Mutations close to the intron-exon border can result in altered splicing variants. In the most prominent intron class in plants, the U2 type introns, both the highly conserved GU donor sequence at the 5'splice site and the highly conserved AG acceptor sequence at the 3 'splice 
site are necessary for the spliceosome to recognize intron-exon borders correctly (Lorković et al., 2000).

In $c r k 7-6$ the acceptor sequence "AG" at the end of intron six is mutated to "AA", making it impossible for the spliceosome to recognize the correct end of intron six. To investigate if the CRK7 transcript is altered in crk7-6, it was amplified from full suppressor plants via RT-PCR and subsequently sequenced. Aligning of the sequencing results revealed that all suppressor plants carried a single base pair deletion in the cDNA (Figure 10A). Most likely, the spliceosome recognized the next AG in the pre-mRNA as acceptor sequence (Figure 10A). This deletion in the mRNA of $c r k 7-6$ results in a frameshift and a premature stop codon leading to a protein truncated within kinase subdomain 10 (Figure 10A).

noce13-34 M3 plants heterozygous for the crk7-6 mutation were infected with E. cruciferarum again and genotyped to investigate if the semi-dominant phenotype co-segregates with the $c r k 7-6$ mutation. Infected plants showed the semi-dominant phenotype (Figure 10B) and genotyping confirmed co-segregation with the $c r k 7-6$ mutation. 


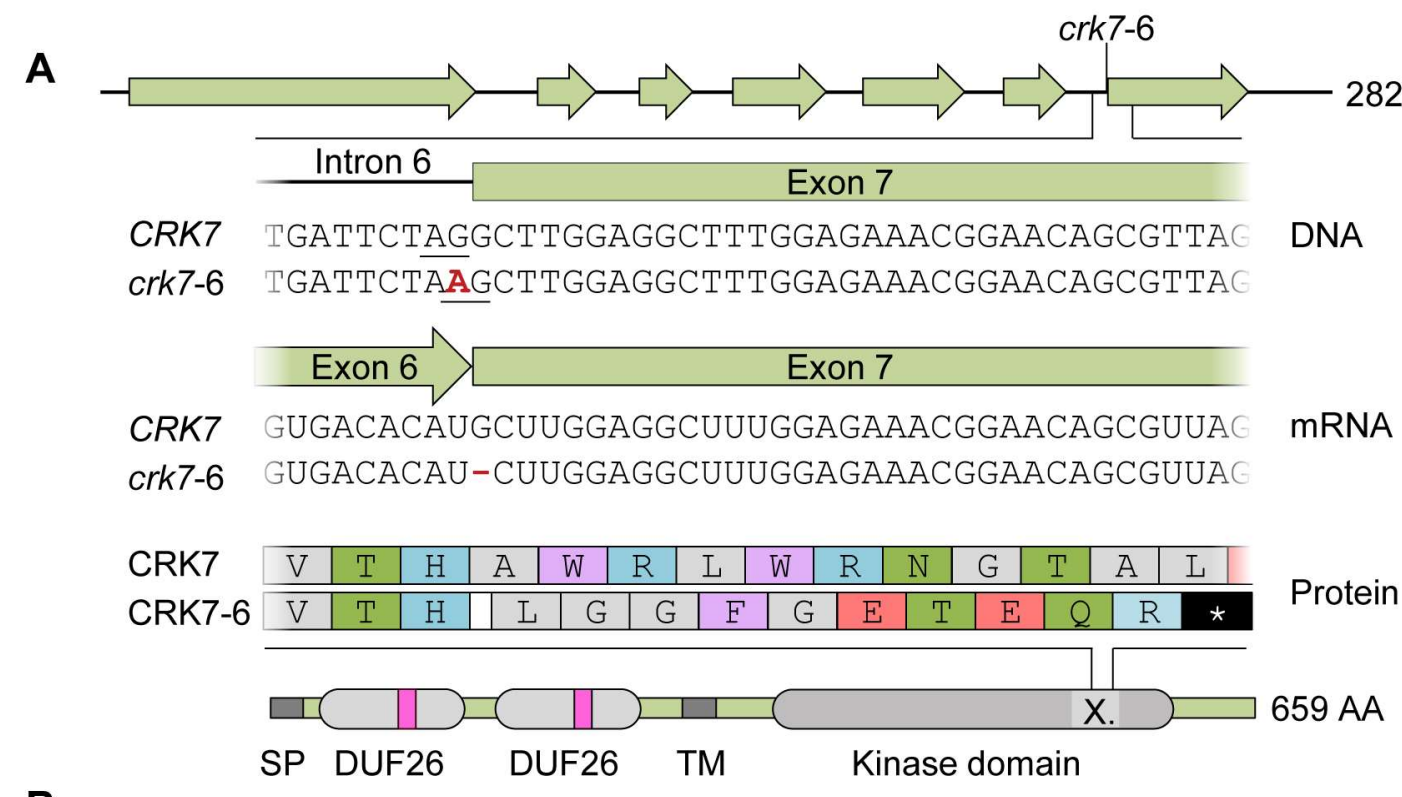

B

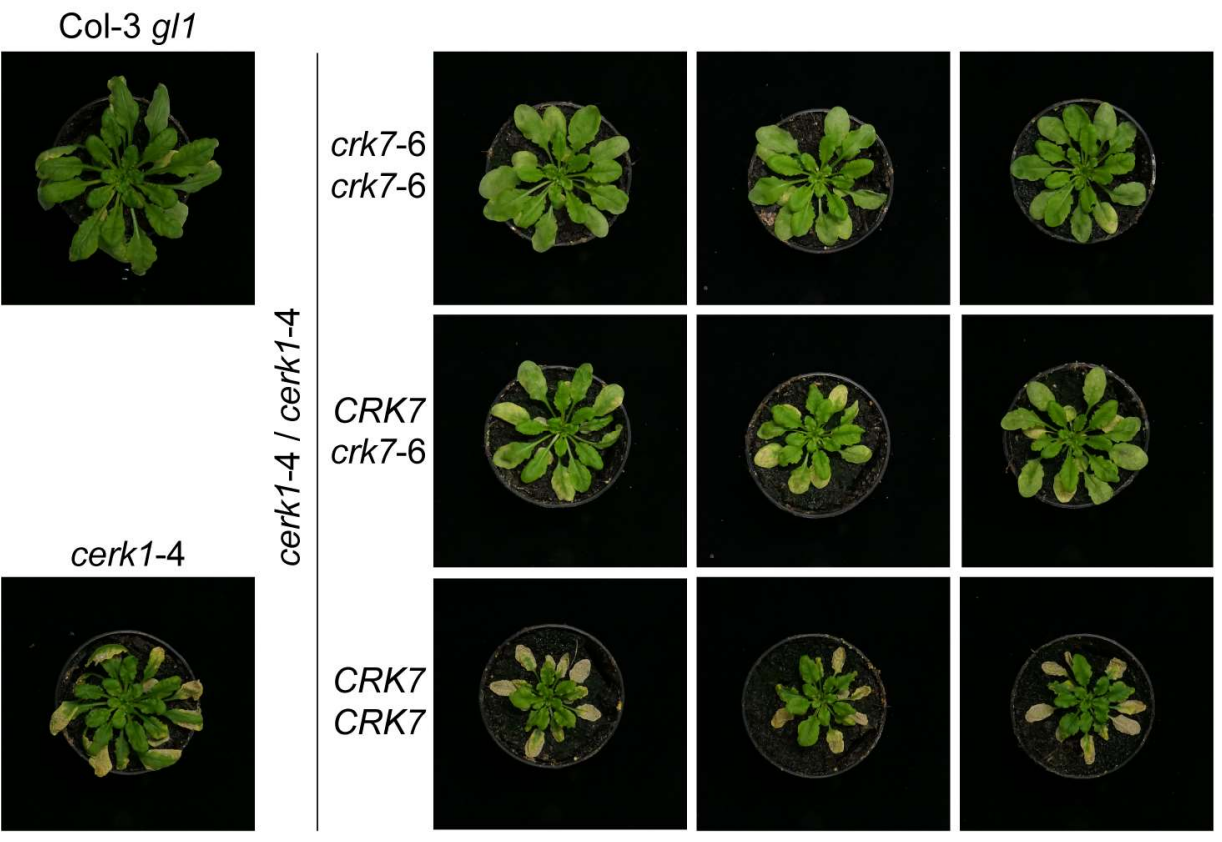

Figure 10: The crk7-6 mutation identified in the cerk1-4 suppressor screen shows a semi-dominant phenotype.

A: Schematic structure of the CRK7 gene, transcript and protein. Exons are depicted as light green arrows. The DNA sequence alignment shows the guanine ${ }^{2314}$ to adenine nucleotide exchange (red) in $c r k 7-$ 6. The spliceosome acceptor sequences (AG) at the end of intron 6 are underlined in the WT and crk7-6 sequences. The $c r k 7-6$ transcript is aligned to the CRK7 wildtype sequence and the missing base pair is highlighted in red. Predicted protein features: Signal peptide (SP), DUF26 domains with the internal C$\mathrm{X}_{8}-\mathrm{C}-\mathrm{X}_{2}-\mathrm{C}$ motif (pink box), transmembrane domain (TM) and kinase domain. The light grey box in the kinase domain highlights kinase subdomain X. Domains were predicted using SMART (http://smart.emblheidelberg.de/, Letunic and Bork, 2018). Color code for amino acids: Polar positive= blue, polar negative $=$ red, polar neutral $=$ green, non-polar aliphatic $=$ grey, non-polar aromatic $=$ purple, ${ }^{*}=$ stop codon. $\mathbf{B}$ : Pictures of E.cruciferarum infected M3 plants and the according controls. Genotypes are indicated left of the images and three representative images per genotype are shown. Pictures were taken $13 \mathrm{dpi}$. 


\subsubsection{Analysis of $C R K 7$ knockout lines generated with the CRISPR/ Cas9 system}

\subsection{Isolation of the cerkl-4 crk7-7 and cerk1-4 crk7-8 full deletion lines}

In all crk7 mutant lines analyzed so far, heterozygous plants showed an intermediate phenotype upon E. cruciferarum infection (3.1.1, 3.1.2.1.2, 3.1.2.2). In all three lines either only an amino acid is exchanged (crk7-4) or a truncated CRK7 protein might be present (crk7-5, crk7-6). These mutated proteins might still fulfill a partial CRK7 function or have dominantnegative effects, leading to the semi-dominant phenotype. As working with a semi-dominant phenotype is rather complex, especially with regard to complementation approaches, it is important to obtain a complete crk7 knockout line where the full-length $C R K 7$ coding sequence is deleted. To achieve this, the CRISPR/ Cas9 system was used. For guide RNA (gRNA) design the regions $500 \mathrm{bp}$ upstream of the ATG and $500 \mathrm{bp}$ downstream of the TGA were used as queries. Different online tools were used for gRNA prediction (2.2.5.2), the results were compared and the three most common hits for the $3^{\prime}$ and $5^{\prime}$ region (A-F in figure 11A) were combined with each other, generating nine plasmids in total. These plasmids were transformed into cerkl-4 plants via floral dip. The analysis in the T1 generation was started with plasmids in which guide RNAs A and F (pl-gRNA-A+F), guide RNAs B and E (plgRNA-B+E) or guide RNAs C and D (pl-gRNA-C+D) were combined. For analysis of the T1 generation, leaf discs of nine Basta ${ }^{\circledR}$ selected T1 plants were pooled and DNA was extracted for PCR based genotyping with oligonucleotides amplifying the whole $C R K 7$ region (oCT131 + oCT132; Figure 11A). In case of a successful CRISPR event, the expected PCR product is much smaller than the WT fragment. Such a PCR product was detected in three plgRNA-A+F and in two pl-gRNA-B+E DNA pools. For these samples the DNA from all nine plants in the pool was isolated separately and analyzed via PCR to identify the plant carrying the $C R K 7$ deletion. Plants either heterozygous or homozygous for $C R K 7$ deletions were isolated and propagated into the T2 generation. As the analysis of these three constructs was already successful, plants transformed with the remaining six CRISPR-constructs were not further investigated.

In the $\mathrm{T} 2$ generation, plants homozygous for the $C R K 7$ knockout were identified via PCR based genotyping (Figure 11B and C). The PCR products obtained with oCT131 and oCT132 were sequenced and sequencing results were aligned to WT CRK7 gDNA (Figure 11D and E). This confirmed that the Cas9 enzyme cut the DNA close to the protospacer adjacent motif (PAM) sequences and the entire coding sequence of $C R K 7$ was deleted. The identified homozygous CRK7 knockout plants were named crk7-7 (Figure 11B and D) and crk7-8 (Figure 
$11 \mathrm{C}$ and E). As a WT control, a PCR reaction with a primer binding within the first exon of CRK7 was performed (oCT131 and oCT25, figure 11A). If a plant is homozygous for the deletion, this should not result in a PCR product. Surprisingly a PCR product (black asterisk in figure $11 \mathrm{~B}$ and C) larger than the WT PCR product was present. This PCR product was sequenced and it turned out to align to $C R K 8$, suggesting that the oligonucleotide oCT25 binds not only to $C R K 7$ but also to the neighboring and closely related gene $C R K 8$. Similar results were observed for crk7-8 (Figure 11C and E). Taken together these results confirm crk7-7 and $c r k 7-8$ as $C R K 7$ knockout lines. Due to time reasons, only crk7-7 was further characterized in E. cruciferaum infection experiments (3.1.2.3.2).

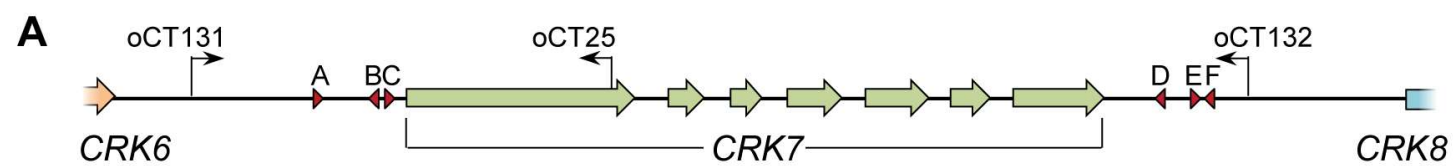

B

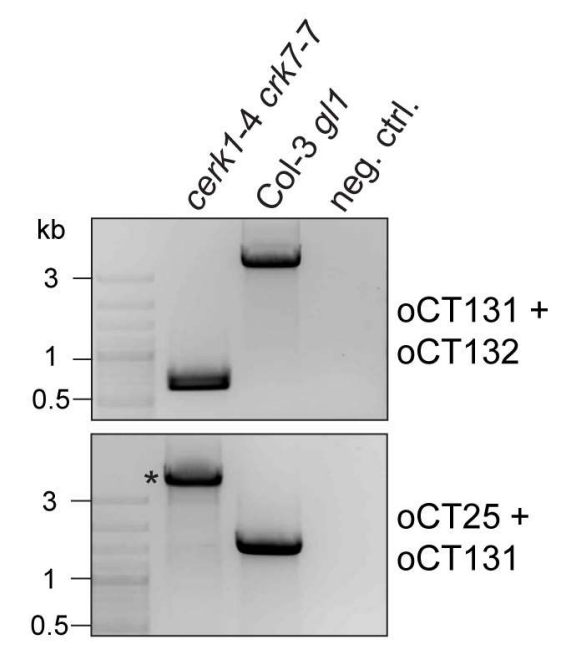

D

CRK7 CTCCCATATTATTTAAGAGGTGGAGAAACAATGATTAAGTTTTTTGGG

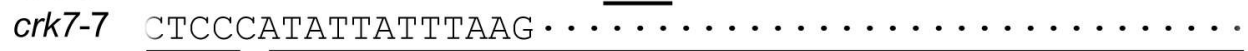

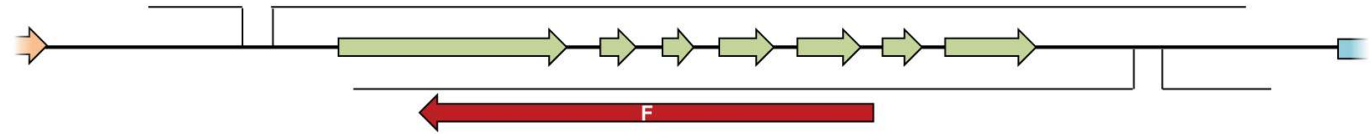

CRK7 GGTGATTTCATGATTTGAAAGTGAAATAAATGAAATGT

crk7-7 ..............................

E

CRK7 AAAGTCGTAACAAACCACCGTATATTTCACGTTAATCAAATAATCAGCA

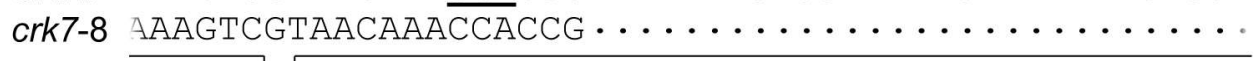

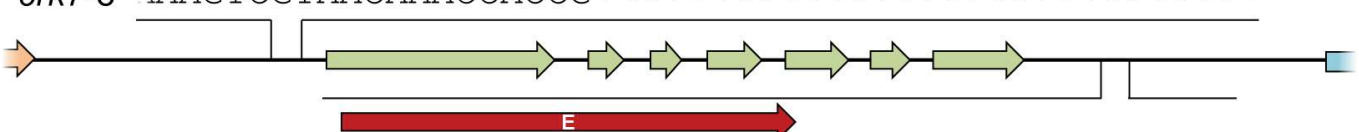

CRK7 GAGGGAAAACAGAGTACAGTGGGATGGTGATTTGCATGATTTGT

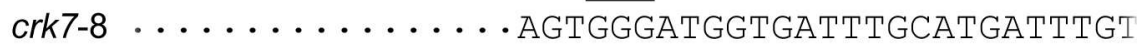


Figure 11: The crk7-7 and crk7-8 knockout mutants generated with the CRISPR/ Cas9 system in this work.

A: Schematic structure of the $C R K 7$ gene as well as upstream and downstream regions. $C R K 7$ exons are depicted with light green arrows. The guide RNAs (gRNAs) are depicted with red arrowheads. Primers used for genotyping are indicated with black arrows. B + C: PCR-based genotyping of crk7-7 (B) and crk7-8 (C) using the oligonucleotides indicated in A. The PCR product highlighted with the asterisk aligns to $C R K 8$. D + E: Sequence alignments of $c r k 7-7$ (D) and $c r k 7-8$ (E) to the $C R K 7$ WT sequence. $C R K 7$ exons are depicted with light green arrows. gRNAs are depicted as red arrows, letters refer to the gRNA overview in A. The protospacer adjacent motif (PAM) is underlined in the sequence alignment.

\subsection{The crk7-7 deletion suppresses the cerk1-4 phenotype}

Segregating T2 plants of cerk1-4 crk7-7 were genotyped using the primers indicated in figure 11A. To examine if crk7-7 shows a semi-dominant or recessive suppressor phenotype upon infection, plants homozygous for cerk1-4 and either homozygous, heterozygous or WT for crk7-7 were infected with E. cruciferarum together with the controls cerkl-4 and Col-3 gll. Pictures of the infected plants were taken 16 dpi (Figure 12). In this infection experiment cerk1-4 itself did not show a strong cell death phenotype. This was observed very rarely before in independent experiments and the reason for this is unclear (E. Petutschnig, personal communication). Nevertheless, T2 plants homozygous for cerk1-4 and WT for CRK7 showed clear cerk1-4 phenotypes. Homozygous cerk1-4 crk7-7 plants developed full suppressor phenotypes with strong E. cruciferarum growth. Plants homozygous for cerkl-4 and heterozygous for crk7-7 clearly showed an intermediate phenotype (Figure 12) as observed before for the other analyzed crk7 mutants. In contrast to the analyzed crk7 mutants identified in the screen or the $c r k 7-5$ T-DNA insertion line, $c r k 7-7$ plants do not contain any mutated or truncated CRK7 versions. Therefore, the most likely explanation for the semi-dominant phenotype is a gene dose effect. 


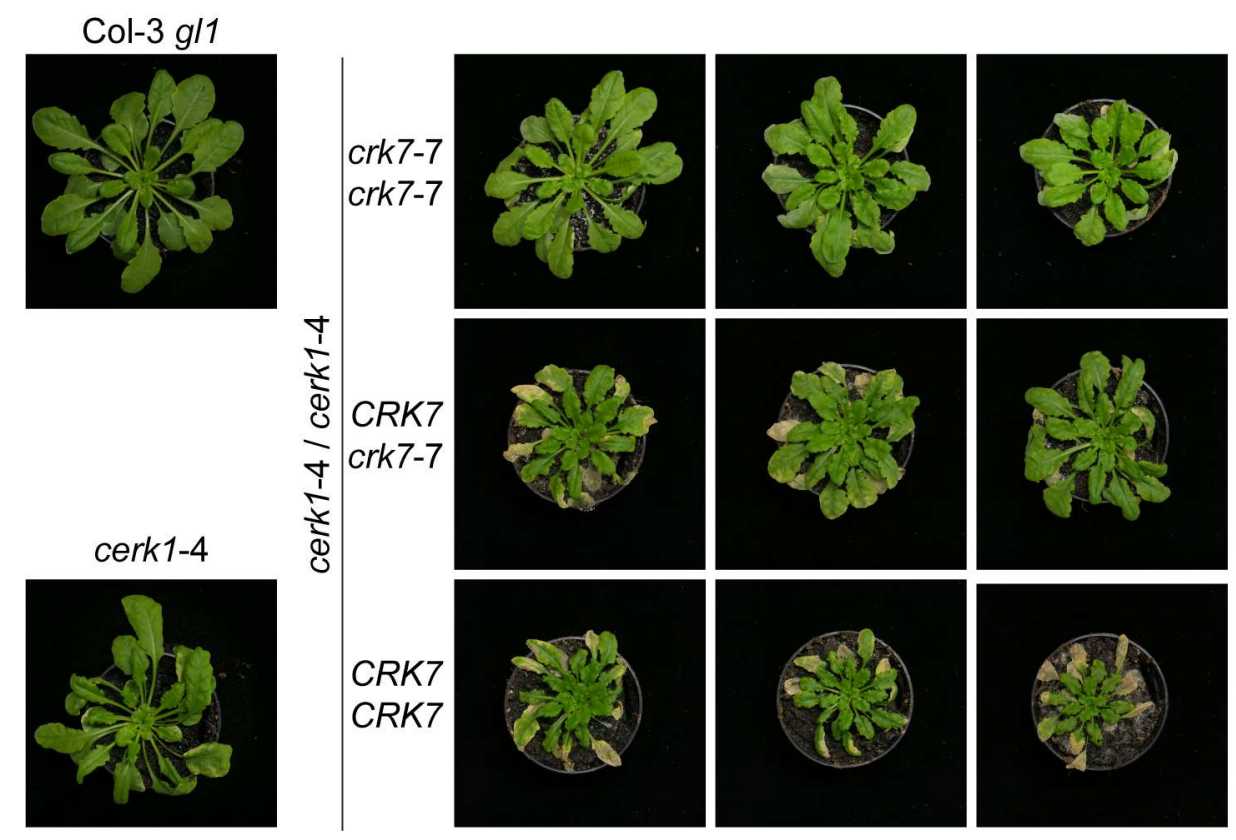

Figure 12: cerk1-4 crk7-7 shows a semi-dominant suppressor phenotype upon infection with $E$. $c r u$ ciferarum.

Segregating cerk1-4 crk7-7 T2 plants were genotyped, randomized with the control plants and infected with E. cruciferarum. Pictures of infected plants and the according controls were taken 16 days post infection (dpi). Genotypes are indicated left of the images and three representative images per genotype are shown.

\section{$\underline{3.1 .3 \mathrm{crk} 7 \text { mutant lines show normal PAMP responses }}$}

Several CRKs were reported to interact with PAMP receptor complexes and contribute to PAMP-triggered immune responses (Yeh et al., 2015; Lee et al., 2017; Yadeta et al., 2017). Therefore flg22 and chitin responses were investigated in $c r k 7$ mutant lines.

The crk7-4 EMS mutant line and the crk7-5 T-DNA line were tested for their ability to induce accumulation of reactive oxygen species (ROS) and phosphorylation of MAPK after flg22 and chitin treatment. For crk7-5, the expression of the PAMP-inducible transcription factors WRKY30 and WRKY53 (Rushton et al., 2010; Cao et al., 2014) was tested additionally.

Col-3 gll, cerkl-4 and Col-0 were included in the experiments as controls as they are the background of crk7-4 (Col-3 gll), cerk1-4 crk7-4 (cerk1-4) and crk7-5 (Col-0) respectively. cerk1-2 and fls2-c knockout lines were used as negative controls.

\subsubsection{1 crk7 mutants show WT-like PAMP induced ROS accumulation}

One of the early responses upon PAMP perception is accumulation of extracellular ROS. In Arabidopsis the NADPH oxidases RBOHD and RBOHF are activated upon PAMP percep- 
tion and generate ROS in the apoplast (Torres and Dangl, 2005). To investigate if the ability to induce ROS accumulation upon PAMP treatment is changed in crk7 mutant lines, a luminol-based assay was performed with either $100 \mathrm{nM}$ flg22, $100 \mu \mathrm{g} / \mathrm{ml}$ chitin or water as negative control (2.2.3.16).

crk7-4 and cerk1-4 crk7-4 plants showed ROS bursts comparable to Col-3 gll and cerkl-4 in response to both chitin and flg22 (Figure 13A). crk7-5 plants were able to accumulate ROS to a level similar to Col-0 after both chitin and flagellin treatment (Figure 13B). As expected, the cerk1-2 knockout line did not react to chitin and the fls2-c knockout line was unable to induce ROS accumulation after flagellin treatment.

As both tested $c r k 7$ mutant lines showed no altered ROS accumulation in response to chitin and flagellin in comparison to the according controls, this suggests that CRK7 does not play a role in extracellular ROS accumulation upon PAMP perception.

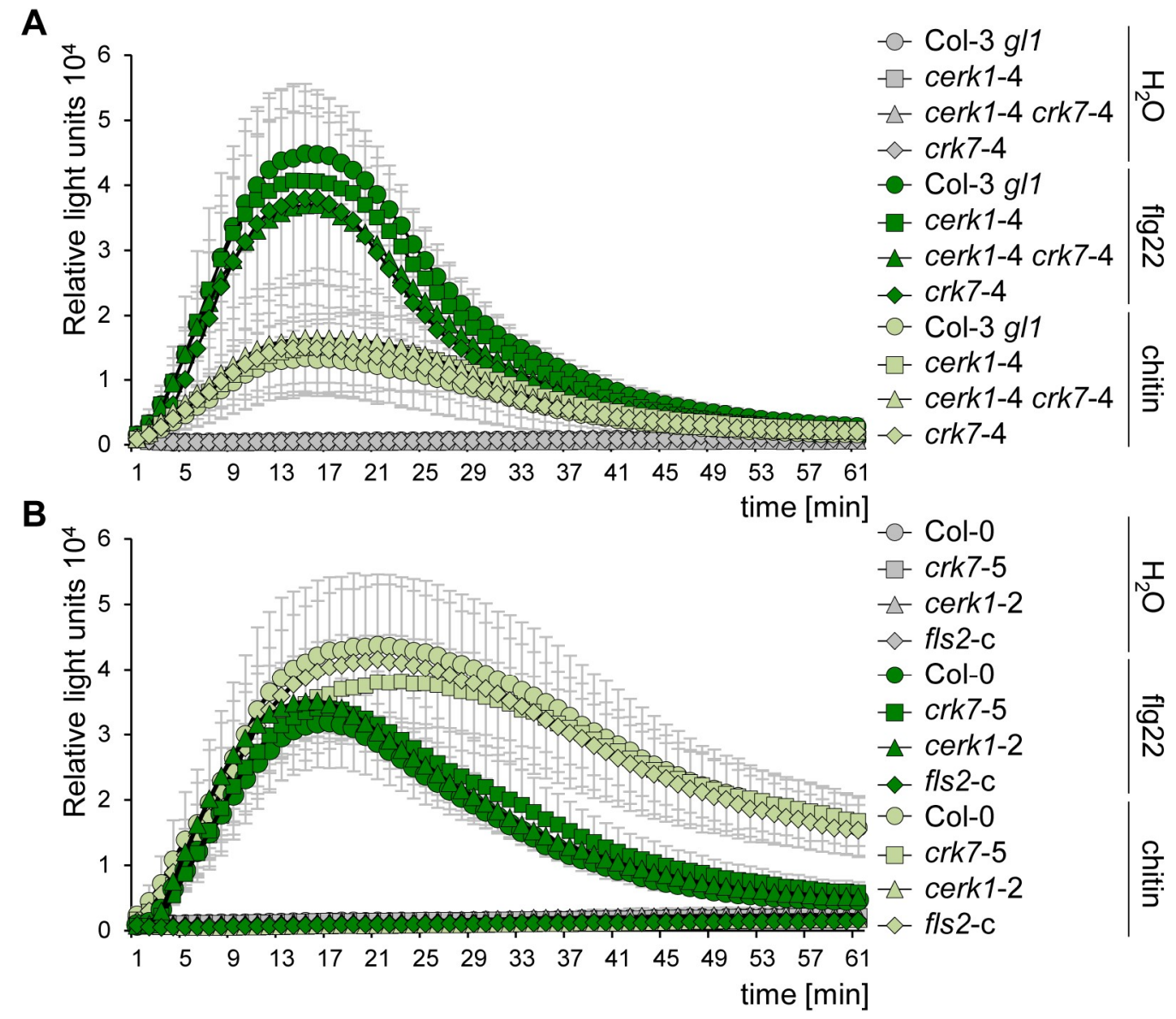

\section{Figure 13: $c r k 7$ mutants show WT-like ROS bursts upon flg22 and chitin treatment.}

A and B: Eight leaf discs per line of five- to seven-week-old Arabidopsis plants were treated with either water (grey), polymeric chitin $(100 \mu \mathrm{g} / \mathrm{ml}$, light green) or flagellin22 (flg22, $100 \mathrm{nM}$, dark green). Relative light units were measured every minute for $60 \mathrm{~min}$ after the respective treatment. Data show mean of eight leaf discs and error bars represent StDev. The experiments were performed in six (A) or two (B) replicates with similar results. Experiment in B was performed by L. Pierdzig during Bachelor thesis supervised by C. Trippel. 


\subsubsection{2 crk7 mutants show WT-like PAMP induced MAPK phosphorylation}

Upon perception of PAMPs by specialized PRRs the signal has to be transduced into the plant cell for effective defense against the pathogen. For this purpose, a MAPK phosphorylation cascade is induced which activates further downstream components of the plant immune system (Rasmussen et al., 2012).

To address the question if MAPK phosphorylation is altered in $c r k 7$ mutants compared to the according WT controls, 14-day-old in vitro grown seedlings were treated for twelve minutes with $10 \mu \mathrm{g} / \mathrm{ml}$ chitin, $50 \mathrm{nM}$ flg22 or medium without PAMPs (2.2.1.1.2). Protein extracts were analyzed via Western Blot (2.2.4.5) using the $\alpha$-p42/44-MAPK antibody that specifically detects phosphorylated MAPKs.

The MAPK phosphorylation in crk7-4, cerkl-4 crk7-4 and crk7-5 was neither altered after chitin nor after flagellin treatment compared to the respective WT controls (Figure 14). As expected, cerk1-2 did not show MAPK activation upon chitin treatment and fls2-c was unable to induce MAPK phosphorylation after flagellin treatment (Figure 14). These results indicate that CRK7 does not play a role in MAPK phosphorylation upon PAMP perception.
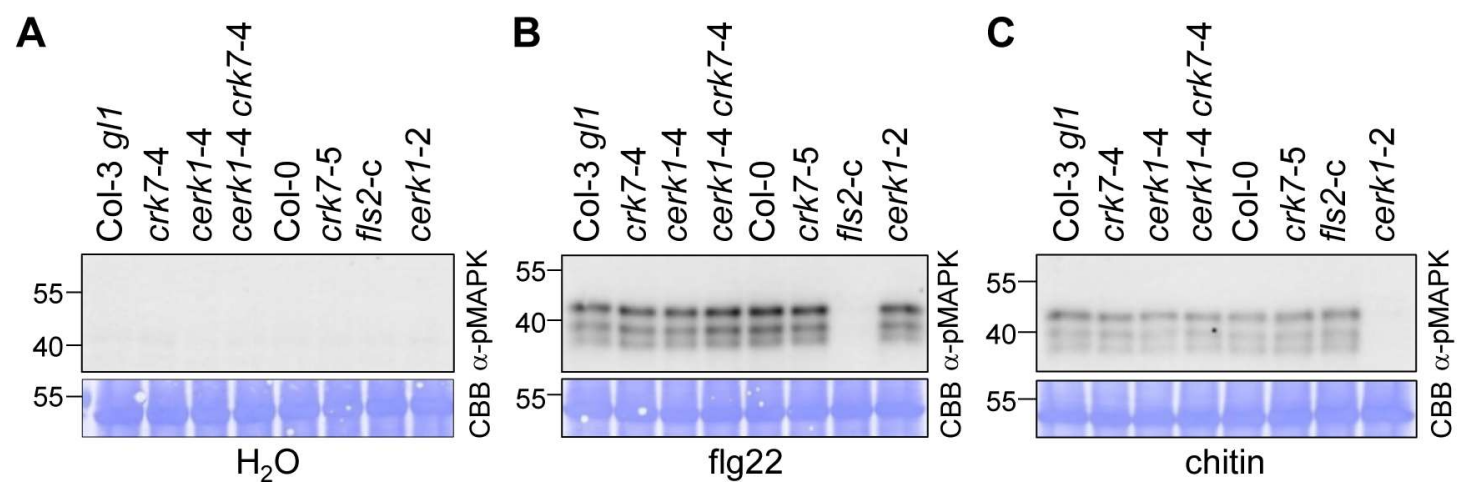

Figure 14: crk7 mutants show WT-like MAPK activation upon flg22 and chitin treatment.

14-day-old seedlings of crk7-5 and crk7-4 mutants and the according controls were treated for 12 minutes with either medium without PAMPs $\left(\mathrm{H}_{2} \mathrm{O}\right), 100 \mathrm{nM}$ flagellin22 (flg22) or $10 \mathrm{mg} / \mathrm{ml}$ polymeric chitin as indicated below the gels. Immunoblotting was performed with the p42/44- $\alpha$-MAPK antibody ( $\alpha$-pMAPK) detecting phosphorylated MAPKs. fls2-c serves as negative control for flg22 perception, cerk1-2 as negative control for chitin perception. CBB: Coomassie Brilliant Blue-stained membranes. This experiment was performed in two (cerk1-4 crk7-4), three (crk7-4) or four (crk7-5) replicates with similar results.

\subsubsection{PAMP-induced WRKY30 and WRKY53 induction is normal in crk7-5}

MAPK cascades are activated rapidly upon PAMP perception and activate further downstream components of the plant immune system. One downstream reaction triggered by phosphorylated MAPKs is the activation of transcription factors which leads to the expres- 
sion of defense genes (Rasmussen et al., 2012). One class of early PAMP induced transcription factors are the WRKY family transcription factors. To investigate if WRKY3O and/ or WRKY53 show an altered expression level in crk7-5 compared to Col-0 upon PAMP treatment, 14-day-old in vitro grown seedlings were treated for 30 minutes with $10 \mu \mathrm{g} / \mathrm{ml}$ chitin, $50 \mathrm{nM}$ flg22 or medium without PAMPs (2.2.1.1.2). RNA was extracted from the treated seedlings and cDNA was used for semi-quantitative PCR (2.2.3.15). ACTIN was included as control. WRKY30 and WRKY53 expression in crk7-5 is induced after both chitin (Figure 15A) and flagellin (Figure 15B) treatment at a level comparable to Col-0. As expected, expression of WRKY30 and WRKY53 is not induced after chitin treatment in cerk1-2 and after flagellin treatment in fls2-c (Figure 15). These results show that knockout of $c r k 7$ does not affect expression of WRKY30 and WRKY53 upon PAMP perception.

A

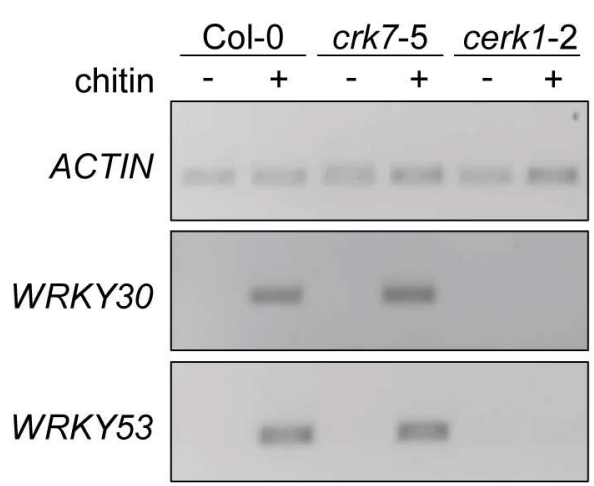

B

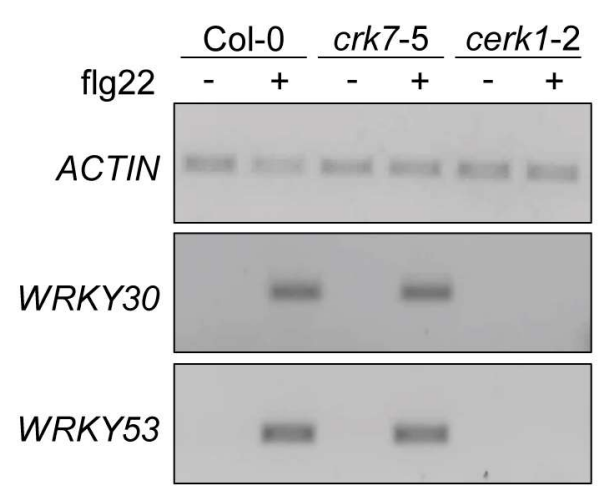

Figure 15: WRKY30 and WRKY53 gene expression is not altered in crk7-5 upon PAMP treatment. 14-day-old seedlings of crk7-5, Col-0 as WT-control, cerk1-2 as negative control for chitin perception and $f l s 2-c$ as negative control for flg22 perception were treated for 30 minutes with either, $10 \mathrm{mg} \mathrm{ml}^{-1}$ polymeric chitin (A) or $100 \mathrm{nM}$ flagellin22 (flg22) (B). Semi-quantitative RT-PCR was performed for ACTIN (control), WRKY30 and WRKY53. The experiment was performed three times with similar results by L. Pierdzig during Bachelor thesis supervised by C. Trippel.

\subsubsection{CRK7 complementation approaches}

All analyzed crk7 mutants show a semi-dominant phenotype upon infection with E. cruciferarum (3.1.1 and 3.1.2). Therefore, dosis-effects of CRK7 protein levels are likely to play an important role for cell death induction. Working with a semi-dominant mutant makes complementation of the mutant phenotype challenging. Nevertheless, complementation, especially with tagged protein versions, is important for further analysis of protein functions.

Therefore, it was tested if CRK7 is able to re-establish cell death or at least the intermediate phenotype in cerk1-4 crk7-4 and/ or cerk1-4 crk7-5 suppressor plants. Additionally, it was investigated if $c r k 7-4$ is able to partially or fully suppress the cell death phenotype in cerk $1-4$ 
plants. In sum and irrespective of the experimental setup, the number of transformants that showed a complementation phenotype was unexpectedly low. Conducted complementation attempts are summarized in the following chapters.

\subsubsection{Complementation by expressing CRK7 in cerk1-4 crk7-4 and cerk1-4 crk7-5}

\subsection{Expression of untagged CRK7 in cerk1-4 crk7-4 and cerk1-4 crk7-5}

Initial complementation experiments were conducted by expressing untagged CRK7 under the endogenous promotor in cerk1-4 crk7-4 plants. These plants express endogenous CRK7-4 in addition to the transgenic CRK7. Therefore, functional complementation would result rather in an intermediate phenotype than in full re-establishment of cell death, depending on the expression level of transgenic CRK7.

In the corresponding experiment, 71 four-week old T1 cerk1-4 crk7-4 plants transformed with $p$ GreenII-0229-pCRK7::CRK7 were infected with E. cruciferarum. 70 plants showed clear suppressor phenotypes upon infection and only one plant fully re-established cell death (Table 14). CRK7 might be either not expressed in the transformed cerk1-4 crk7-4 plants or the expression level might be too low to induce cell death. Comparison of $C R K 7$ and crk7-4 transcript abundance by RT-PCR was attempted several times but was unfortunately unsuccessful (data not shown). Problems faced were generally low $C R K 7$ expression levels and the high similarity between $C R K \mathrm{~s}$ also on nucleotide level, making the design of specific and efficient primers difficult.

To obtain higher expression levels, CRK7 was expressed from the CaMV 35S promoter in cerk1-4 crk7-4 as well as cerk1-4 crk7-5 plants. Seven cerk1-4 crk7-4 plants transformed with $p$ GreenII-Kan-p35S::CRK7 were identified and infected with E. cruciferarum. Two of these seven re-established cerk1-4-like cell death, the remaining five plants showed a full suppressor phenotype. One of the complemented plants died without seed production but the other one ( $p 35 S:: C R K 7$ in cerk1-4 crk7-4 \#5) was investigated in the T2 generation. Seven of the 19 investigated T2 plants re-established cerk1-4-like cell death upon E. cruciferarum infection (Table 14).

The $p 35 S:: C R K 7$ construct was additionally transformed into cerk1-4 crk7-5, which harbours a T-DNA insertion in CRK7 (3.1.2.1). Twelve T1 transformants were obtained of which four re-established cell death in response to E. cruciferarum infection. One line died without seed production, but the remaining three lines were examined in the T2 generation. In one line (p35S::CRK7 in cerk1-4 crk7-5 \#7) none of the 19 investigated T2 plants re-established cell 
death upon E. cruciferarum infection. For the other two lines (\#1 and \#8) 15 of 19 T2 plants (line \#1) and 9 of 19 T2 plants (line \#8) developed cell death (Table 14). Therefore, reestablishment of cell death was more successful upon $C R K 7$ overexpression than after expression with the native promotor, suggesting that a higher $C R K 7$ expression level is necessary for complementation.

Table 14: Overview of the analyzed lines for complementation transformed with untagged CRK 7 .

\begin{tabular}{|c|c|c|c|c|c|c|}
\hline \multirow{2}{*}{ Construct } & \multirow{2}{*}{ Background } & \multicolumn{2}{|c|}{$\mathrm{T} 1$ plants } & \multicolumn{3}{|c|}{ T2 plants } \\
\hline & & analyzed & cell death & lines & analyzed & cell death \\
\hline$p C R K 7:: C R K 7$ & cerk1-4 crk7-4 & 71 & $1(1.4 \%)$ & - & & - \\
\hline$p 35 S:: C R K 7$ & cerk1-4 crk7-4 & 7 & $2^{\mathrm{a}}(28.6 \%)$ & $\# 5$ & 19 & $7(37 \%)$ \\
\hline \multirow{3}{*}{$p 35 S:: C R K 7$} & \multirow{3}{*}{ cerk1-4 crk7-5 } & \multirow{3}{*}{12} & \multirow{3}{*}{$4^{\mathrm{a}}(33.3 \%)$} & $\# 1$ & 19 & $15(79 \%)$ \\
\hline & & & & $\# 7$ & 19 & $0(0 \%)$ \\
\hline & & & & $\# 8$ & 19 & $9(47 \%)$ \\
\hline
\end{tabular}

a one line died without seed production

3.1.4.1.2 Expression of Citrine- or FLAG-tagged CRK7 in cerk1-4 crk7-4 and cerk1-4 crk7-5 For analysis of protein function, localization and interaction, a functional tagged CRK7 protein version that complements the cerkl-4 suppressor phenotype is necessary. Therefore, cerk1-4 crk7-4 plants transformed with pGreenII-0229-pCRK7::CRK7-Citrine were analyzed. Four-week old plants were infected with E. cruciferarum. Out of 34 cerk1-4 crk7-4 plants transformed with $p C R K 7:: C R K 7$-Citrine eight plants showed intermediate and 26 plants a cerk1-4 suppressor phenotype (Table 15). Microscopy was done for all plants at different time points after infection, but no CRK7-Citrine microscopy signals were detectable. In Western Blot analysis using the $\alpha$-GFP antibody a CRK7-Citrine signal was detected for some plants, but there was no correlation found between CRK7-Citrine expression and phenotype upon infection. Therefore, it cannot be ruled out that the observed intermediate phenotypes are due to a lower E. cruciferarum spore load on these plants.

For overexpression, pGreenII-0229-p35S::CRK7-Citrine was cloned and transformed in cerk1-4 crk7-4 plants. Four-week old T1 transformants were infected with E. cruciferarum. Microscopy was done with cerk1-4 crk7-4 plants transformed with CRK7-Citrine at different time points upon infection. For some plants a CRK7-Citrine microscopy signal was detectable, confirming expression of CRK7-Citrine in those lines. Additionally, expression was confirmed via Western Blot. However, none of the cerk1-4 crk7-4 plants expressing CRK7Citrine from the $35 S$ promoter showed any cerk1-4-like cell death (Table 15). This is in con- 
trast to untagged CRK7 and could mean that fusion to the Citrine tag interferes with CRK7 protein function. Some T1 plants expressing CRK7-Citrine at a very high level (confirmed by microscopy and Western Blot, data not shown), developed smaller and crinkled leaves, leading to a dwarf phenotype. This phenotype was observed consistently in T1 and also T2 plants with a strong CRK7-Citrine microscopy signal.

The p35S::CRK7-Citrine construct was also transformed into cerk1-4 crk7-5. 28 transformants were analyzed in the T1, but in none of these plants a CRK7-Citrine fluorescence signal was detected and all plants showed a cerk1-4 suppressor phenotype upon infection with E. cruciferarum (Table 15). Western Blot analysis revealed that the construct was expressed in only one out of 13 analyzed plants.

As a larger tag like Citrine might interfere with protein function, CRK7 was additionally cloned with a $3 \times$ FLAG tag under the native and $35 S$ promotors. Both constructs were transformed into cerk1-4 crk7-5 and T1 plants were infected with E. cruciferarum. However, none of the analyzed plants showed any macroscopically visible cell death upon infection, despite the fact that expression of $p 35 S:: C R K 7-F L A G$ was confirmed via Western Blot using the $\alpha$ FLAG antibody (data not shown).

Table 15: Overview of analyzed lines for complementation transformed with CRK7Citrine (Cit) or CRK7-FLAG constructs.

\begin{tabular}{|c|c|c|c|c|c|}
\hline \multirow[b]{2}{*}{ Construct } & \multirow[b]{2}{*}{ Background } & \multirow[b]{2}{*}{$\mathrm{T} 1$ plants analyzed } & \multicolumn{3}{|c|}{ Phenotype of T1 plants } \\
\hline & & & Cell death & Intermediate $^{a}$ & $\begin{array}{l}\text { No cell } \\
\text { death }^{\text {a }}\end{array}$ \\
\hline$p C R K 7: \because C R K 7-C i t$ & cerk1-4 crk7-4 & 34 & - & $8(-)$ & $26(-)$ \\
\hline p35S::CRK7-Cit & cerk1-4 crk7-4 & 25 & - & $1(-)$ & $\begin{array}{l}6(+) \\
18(-)\end{array}$ \\
\hline p35S::CRK7-Cit & cerk1-4 crk7-5 & 28 & - & - & $28(-)$ \\
\hline$p C R K 7:: C R K 7-F L A G$ & cerk1-4 crk7-5 & 30 & - & - & 30 \\
\hline p35S::CRK7-FLAG & cerk1-4 crk7-5 & 38 & - & - & 38 \\
\hline
\end{tabular}

${ }^{\mathrm{a}}$ Citrine confocal microscopy signal $(-)=$ not detectable, $(+)=$ detectable

\subsubsection{Complementation by expressing CRK7-4 in cerk1-4}

\subsection{Expression of untagged CRK7-4 in cerk1-4}

As the classical complementation approach was not successful for CRK7 (3.1.4.1), it was investigated if the cerkl-4 phenotype could be suppressed by expression of the amino acid exchange mutant protein CRK7-4. This approach is based on the assumption that ectopic ex- 
pression of CRK7-4 may exert a dominant negative effect and might therefore outcompete the function of native CRK7.

39 cerk1-4 plants transformed with pGreenII-0229-pCRK7::crk7-4 were infected with E. cruciferarum but none of them showed a full or intermediate suppressor phenotype (Table 16). A possible reason is that $c r k 7-4$ expression was too low in those lines. As described in 3.1.4.1.1 for $C R K 7$, also for $c r k 7-4$ no robust expression data could be generated.

To achieve higher expression levels, pGreenII-Kan-p35S::crk7-4 was cloned and transformed into cerk1-4. Two of four T1 plants showed cell death upon infection, the other two were able to suppress cell death successfully (Table 16). Again no robust $C R K 7$ expression data could be generated, hence it is not known if transgene expression correlates with the observed phenotypes.

Table 16: Overview of the analyzed cerk1-4 plants transformed with untagged crk 7-4.

\begin{tabular}{llcccc}
\hline \multirow{2}{*}{ Construct } & \multirow{2}{*}{ Background } & T1 plants analyzed & \multicolumn{3}{c}{ Phenotype of T1 plants } \\
\cline { 4 - 6 } & & & Cell death & Intermediate & No cell death \\
\hline$p C R K 7::$ crk7-4 & cerk1-4 & 39 & 39 & - & - \\
\hline$p 35 S::$ crk7-4 & cerk1-4 & 4 & 2 & - & 2 \\
\hline
\end{tabular}

\subsection{Expression of CRK7-4-Citrine in cerk1-4}

crk7-4 was cloned with a citrine-tag for localization studies both under the native and the $35 \mathrm{~S}$ promotor. These constructs were transformed into cerk1-4 plants and four-week old transformants were infected with E. cruciferarum. Similar to $p C R K 7:: C R K 7$-Citrine, also for pCRK7::crk7-4-Citrine expression of the construct could be confirmed via Western Blot analysis, but no CRK7-4-mCitrine fluorescence signal was detectable (data not shown). 40 T1 plants were analyzed but none of them suppressed the cerk1-4 cell death phenotype (Table 17). Expression of CRK7-4-mCitrine in these lines might be simply too low to outcompete native CRK7.

Therefore, cerk1-4 plants transformed with p35S::crk7-4-Citrine were analyzed. As observed before for $p 35 S:: C R K 7-C i t r i n e$, also a CRK7-4-Citrine fluorescence signal was detectable in some T1 plants. All twelve isolated T1 plants with CRK7-4-Citrine fluorescence signal showed cell death upon infection whereas surprisingly all 22 isolated T1 plants without CRK7-4-Citrine fluorescence signal showed a cerk1-4 suppressor phenotype. In these plants most likely native $C R K 7$ as well as transgenic crk7-4-Citrine were silenced (Table 17). 
To prove this hypothesis, $p 35 S:: C R K 7-C i t r i n e$ was transformed into cerk1-4 and T1 plants were analyzed upon E. cruciferarum infection. Expression of CRK7-Citrine in cerk1-4 plants which natively also express untagged CRK7 should not result in suppressor phenotypes unless silencing effects occur. In nine T1 plants expression of the construct was confirmed via confocal microscopy and these plants show cell death as expected. In 23 additional T1 plants no CRK7-Citrine fluorescence signal was detectable, but they showed a cerk1-4 suppressor phenotype (Table 17). This supports the hypothesis that cerk1-4 cell death suppression in

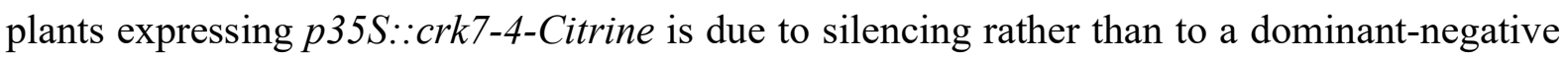
effect of the transgenic protein.

Table 17: Overview of the analyzed cerk1-4 plants transformed with crk7-4-Citrine(Cit)/ CRK 7-Cit.

\begin{tabular}{|c|c|c|c|c|c|}
\hline \multirow{2}{*}{ Construct } & \multirow{2}{*}{ Background } & \multirow{2}{*}{ T1 plants analyzed } & \multicolumn{3}{|c|}{ Phenotype of T1 plants } \\
\hline & & & Cell death ${ }^{a}$ & Intermediate & No cell death ${ }^{a}$ \\
\hline pCRK7::crk7-4-Cit & cerk1-4 & 40 & $40(-)$ & - & - \\
\hline p35S::crk7-4-Cit & cerk1-4 & 34 & $12(+)$ & - & $22(-)$ \\
\hline p35S::CRK7-Cit & cerkl-4 & 31 & $9(+)$ & - & $23(-)$ \\
\hline
\end{tabular}

${ }^{a}$ Citrine confocal microscopy signal $(-)=$ not detectable, $(+)=$ detectable

\subsubsection{Analysis of CRK7 and CRK7-4 subcellular localization}

CRK7 has the predicted structural organization of a classical receptor-like kinase with a signal peptide, an extracellular domain harboring two DUF26 domains, a transmembrane domain and an intracellular kinase domain. Therefore, localization at the plant plasma membrane is expected for CRK7. To investigate localization of CRK7 and CRK7-4, confocal microscopy with fluorescent fusion proteins was performed in N. benthamiana and Arabidopsis.

\subsubsection{Analysis of CRK7-Citrine and CRK7-4-Citrine subcellular localization}

3.1.5.1.1 CRK7-Citrine and CRK7-4-Citrine subcellular localization in transiently transformed Nicotiana benthamiana

Although the CRK7-Citrine and crk7-4-Citrine constructs were not able to re-establish either the cerk1-4 or suppressor phenotype in the according mutant backgrounds (3.1.4), these constructs were used for localization studies in $N$. benthamiana plants transiently expressing the constructs. Confocal microscopy was performed two days post infiltration. 
CRK7-Citrine localized to the cell periphery, a reticulate structure likely to be the ER and to the nuclear envelope (Figure 16). Cells with very low expression levels predominantly showed signals in the cell periphery, however hardly any cells with a pure cell periphery signal were detectable. The stronger the fluorescence, the more ER and nuclear envelope localization was observed. Additionally, round structures of very high fluorescence and different sizes were present in $N$. benthamiana plants infiltrated with CRK7-Citrine (Figure 16). The presence of these round structures correlated with enhanced CRK7-Citrine expression levels as they were more abundant in p35S::CRK7-Citrine infiltrated plants than in N. benthamiana plants infiltrated with $p C R K 7:: C R K 7-C i t r i n e$.

Interestingly, CRK7-4-Citrine was exclusively detectable in the ER and in the nuclear envelope independent of signal strength (Figure 16). Moreover, fluorescence intensities were generally higher in cells producing CRK7-4-Citrine under control of the $p 35 S$ promoter if compared to constructs harboring the native CRK7 promoter (Figure 16).

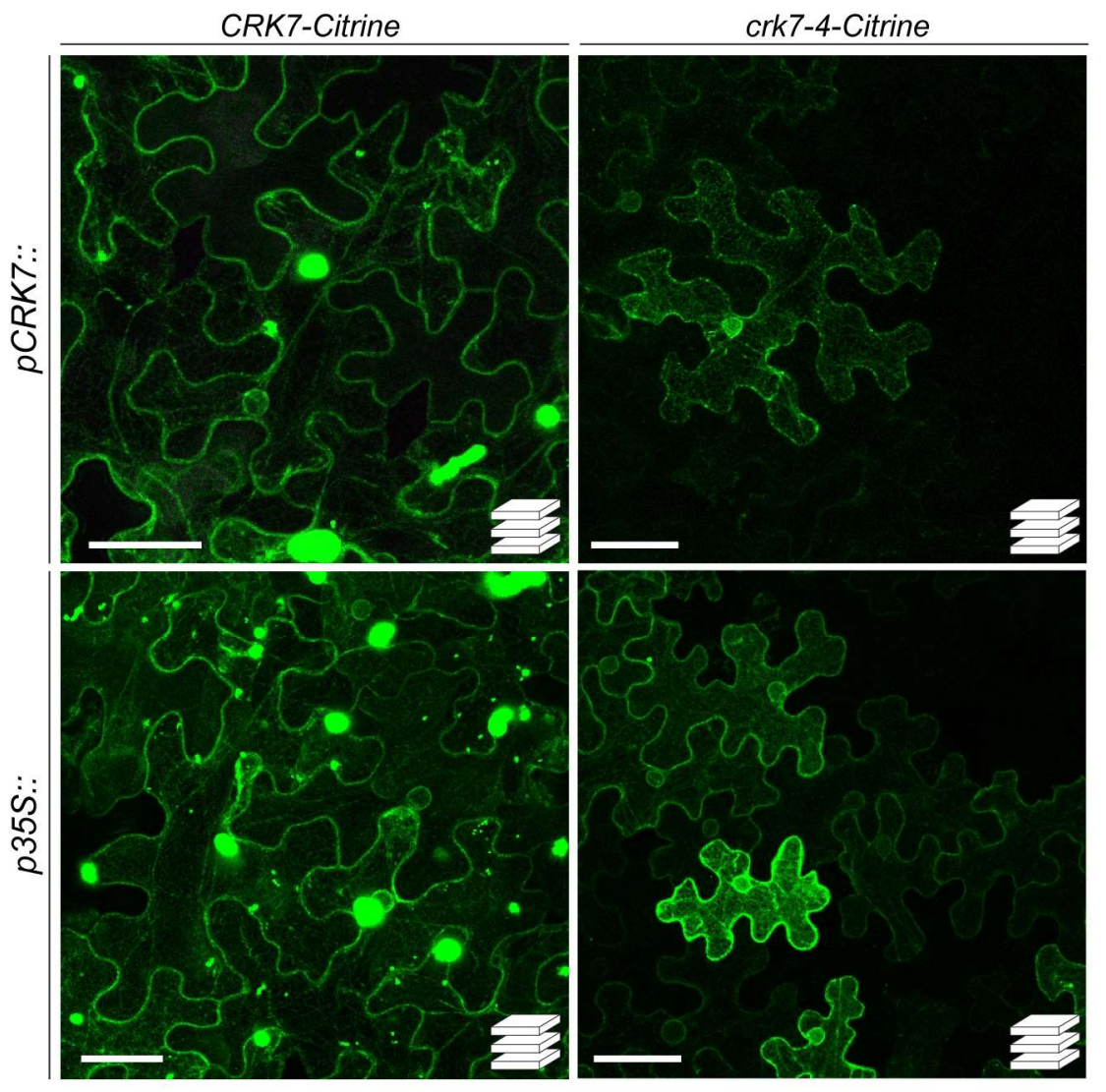

Figure 16: Localization of CRK7-Citrine and CRK7-4-Citrine after transient expression in N. benthamiana.

A. tumefaciens cultures harbouring constructs for expression of CRK7-Citrine (Cit) or crk7-4-Cit under the control of native or $p 35 S$ promotors were infiltrated into $N$. benthamiana. Confocal microscopy (Leica SP5) was performed 2 dpi. Images are representative maximum projections (z-stack size: 17-25 $\mu \mathrm{M}$ ). Scale bar $=50 \mu \mathrm{M}$ 
3.1.5.1.2 CRK7-Citrine and CRK7-4-Citrine subcellular localization in stably transformed Arabidopsis

Confocal microscopy was performed on Arabidopsis cerk1-4 T1 plants stably expressing pCRK7::CRK7/crk7-4-Citrine or p35S::CRK7/crk7-4-Citrine constructs at different time points after E. cruciferarum inoculation. In contrast to the observations in $N$. benthamiana (3.1.5.1.1), fluorescence signals for the $p C R K 7$ constructs could not be observed in Arabidopsis. However, expression of CRK7/CRK7-4-Citrine could be confirmed in Western Blots (data not shown).

Strong fluorescence signals were detectable in uninfected as well as in E. cruciferarum infected p35S::CRK7/crk7-4-Citrine expressing plants. Like in N. benthamiana, CRK7-4Citrine localized to the ER structure only, whereas CRK7-Citrine localized to the cell periphery, ER and nuclear envelope. Additionally the highly fluorescent round structures were present in cerk1-4 plants expressing p35S::CRK7-Citrine. These resemble so-called organized smooth endoplasmatic reticulum structures (OSERs; Snapp et al., 2003; Ferrero et al., 2015), which are protein aggregates in the ER and might not represent the native CRK7 localization. Formation of these structures might also partially explain the problems observed concerning CRK 7 complementation as protein accumulated in OSER structures might be unable to fulfill their functions in cell death induction (3.1.4).

For experiments investigating CRK7 protein function, it would be helpful to have plants expressing CRK7 to a detectable level but not reaching the threshold for the development of aggregates. Therefore, microscopy was repeated in the T2 generation with uninfected plants. For this, three independent lines expressing either p35S::CRK7-Citrine or p35S::crk7-4Citrine were chosen. As expected, all T2 CRK7-4-Citrine plants analyzed showed ER localization independent of signal strength (Figure 17A). For p35S::CRK7-Citrine plants, microscopy was performed with leaves of different ages. The brightly fluorescent OSER-like structures appeared to be more abundant in older leaves than in younger ones (Figure 17B). 
A p35S.::crk7-4-Citrine in cerk1-4
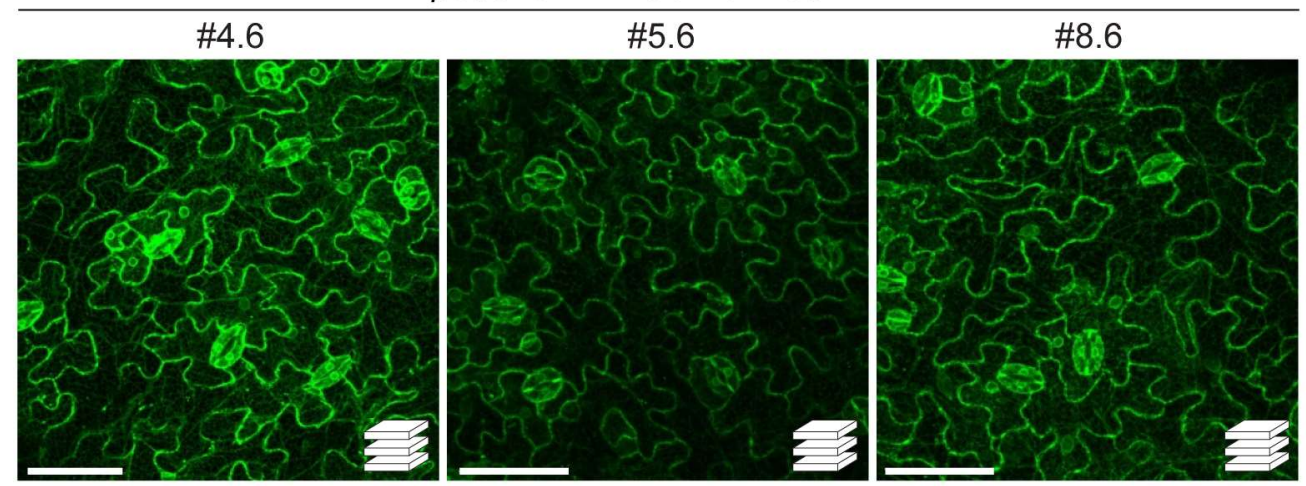

B p35S::CRK7-Citrine in cerk1-4

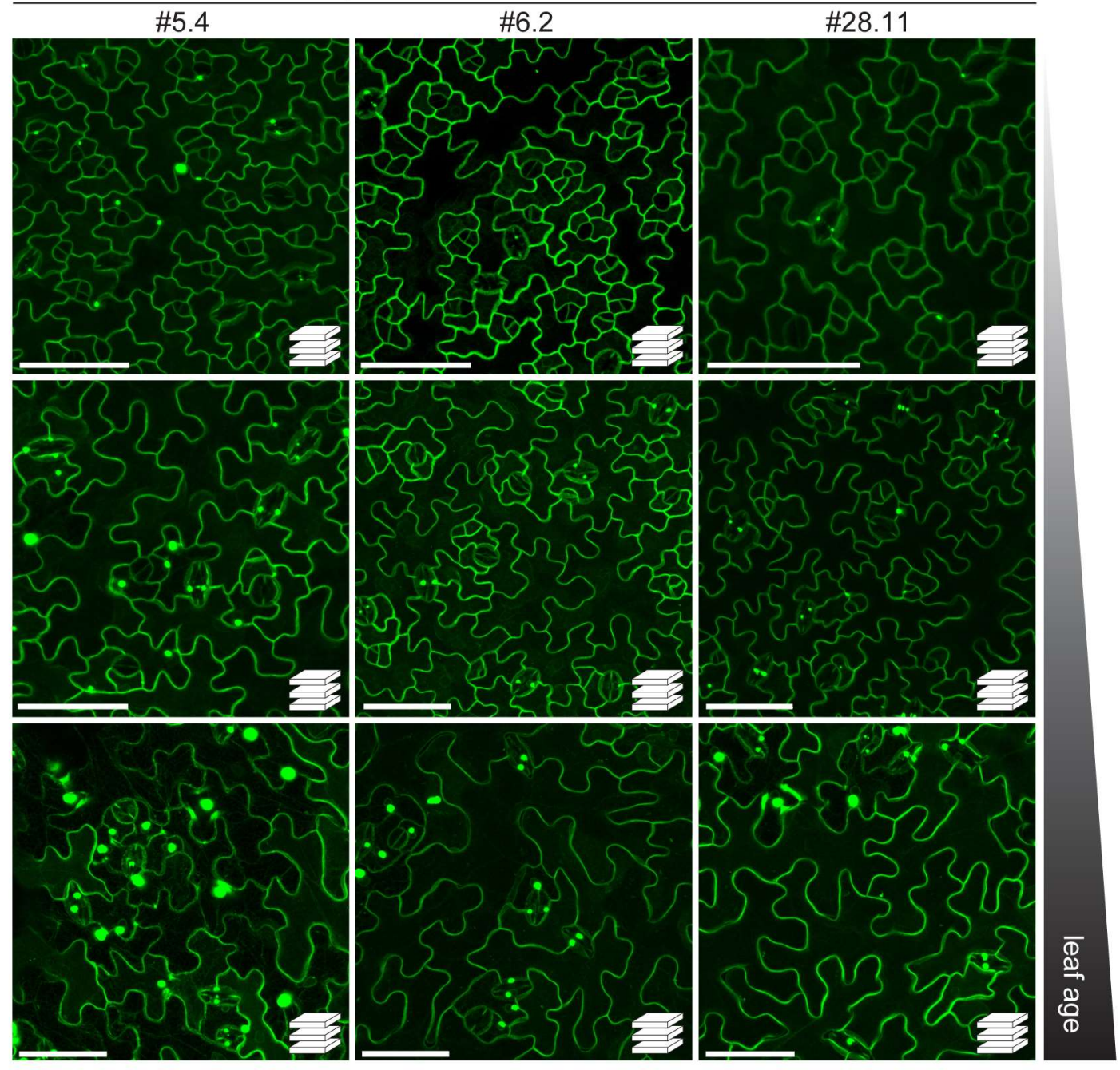

Figure 17: Localization of CRK7-Citrine and CRK7-4-Citrine in cerk1-4.

Confocal microscopy (Leica SP5) was performed with uninfected four-week old stably transformed Arabidopsis T2 plants expressing CRK7-4-Citrine (A) or CRK7-Citrine (B) from the $35 \mathrm{~S}$ promoter. Line numbers (\#) are indicated above to the images. In (B) leaves of different ages were investigated, increasing leaf age is indicated by a triangle on the right side of the figure. Images are representative maximum projections (z-stack size: $6-12 \mu \mathrm{M}$ ). Scale bar $=50 \mu \mathrm{M}$. 
3.1.5.2 Analysis of CRK7-mCitrine and CRK7-4-mCitrine subcellular localization

In localization studies with CRK7-Citrine fusions, round brightly fluorescent OSER-like structures were observed (3.1.5.1). The Citrine-tag can form weak dimers (Cranfill et al., 2016). Dimerization might have an impact on CRK7-Citrine localization and potentially could cause or enhance aggregate formation. Therefore, CRK7 and CRK7-4 localization studies were repeated with proteins fused to mCitrine, a monomeric Citrine variant (Cranfill et al., 2016).

3.1.5.2.1 CRK7-mCitrine and CRK7-4-mCitrine subcellular localization in transiently transformed Nicotiana benthamiana

CRK7-mCitrine or crk7-4-mCitrine were transiently expressed under the native or $35 \mathrm{~S}$ promotors in $N$. benthamiana and confocal microscopy was performed two days post infiltration. As observed for CRK7-4-Citrine (3.1.5.1), also CRK7-4-mCitrine localized to the ER independent of signal intensity (Figure 18A). To visualize signal differences more clearly, intensity was displayed with a false color look-up-table (Figure 18).

In $N$. benthamiana plants expressing CRK7-mCitrine under control of the CRK7 promoter, many cells showed fluorescence signal only in the cell periphery (Figure 18B). This suggests that indeed dimerization of Citrine-tags had an impact on CRK7-Citrine localization. However, in cells expressing CRK7-mCitrine at higher levels, fluorescence was also observed in the ER. The bright round fluorescent structures seen with CRK7-Citrine (3.1.5.1) were never observed with CRK7-mCitrine, but more angularly shaped aggregates were present in some cells with high fluorescence. Nevertheless, it was very clear that in a higher number of cells CRK7-mCitrine microscopy signal was only detectable at the cell periphery compared to the results observed for CRK7-Citrine (Figure 18B).

In general, fluorescence intensities in plants infiltrated with $p 35 S$-constructs were higher than with $C R K 7$-promoter constructs (Figure 18). Consequently, a lower number of cells with pure CRK7-Citrine cell periphery microscopy signal was observed as in most cells CRK7-Citrine localized to the ER and/ or aggregates were formed (Figure 18). 
A
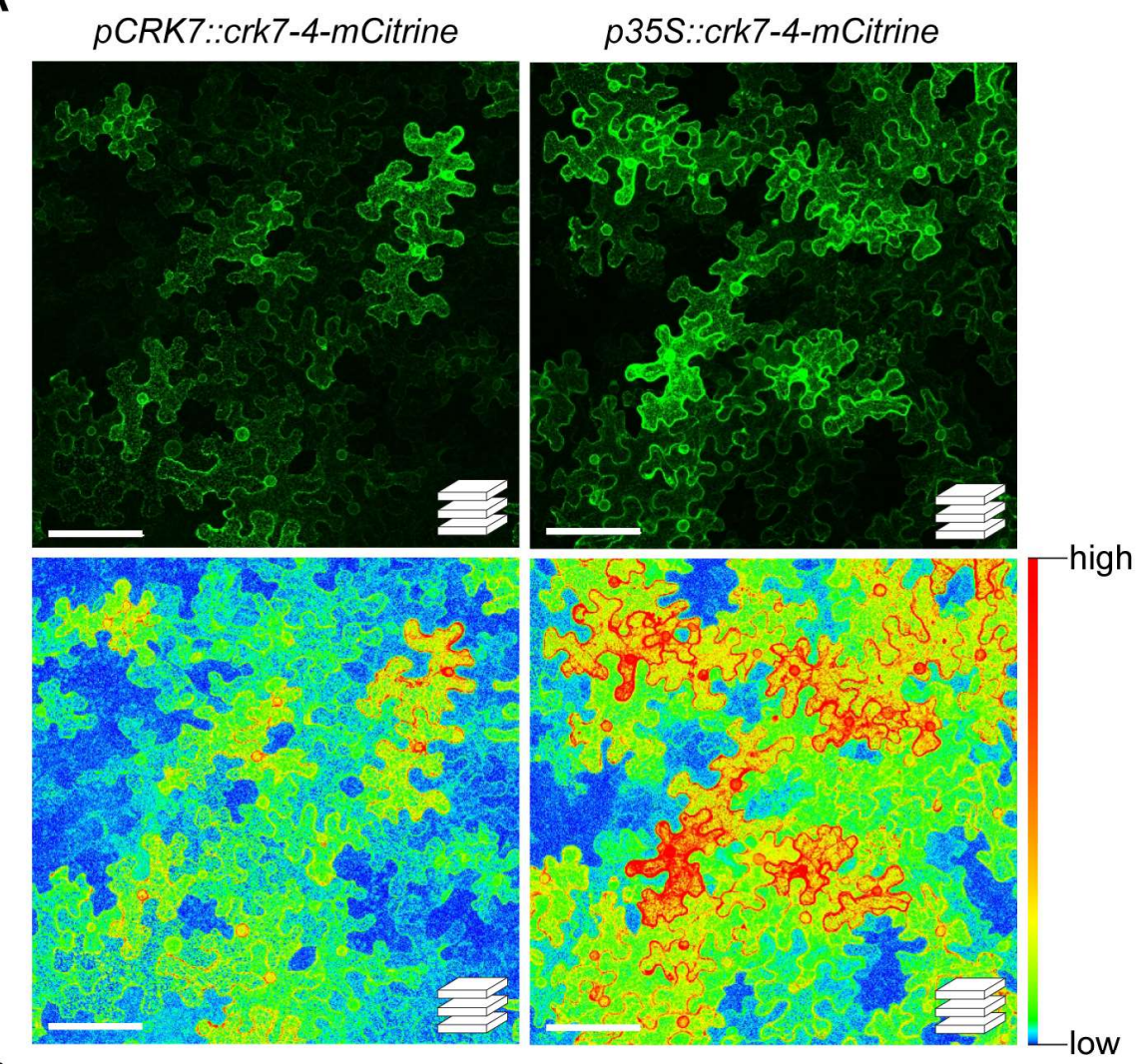

B

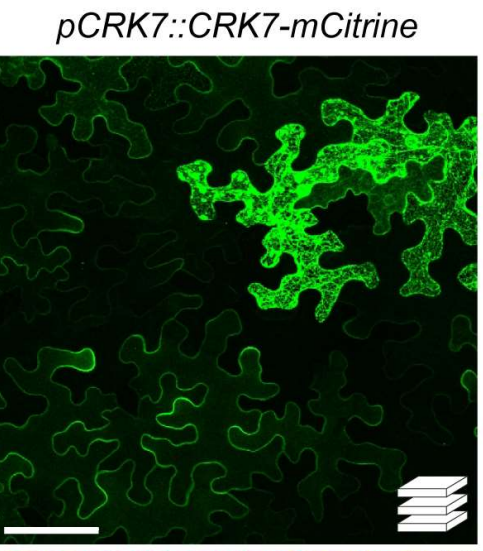

p35S::CRK7-mCitrine
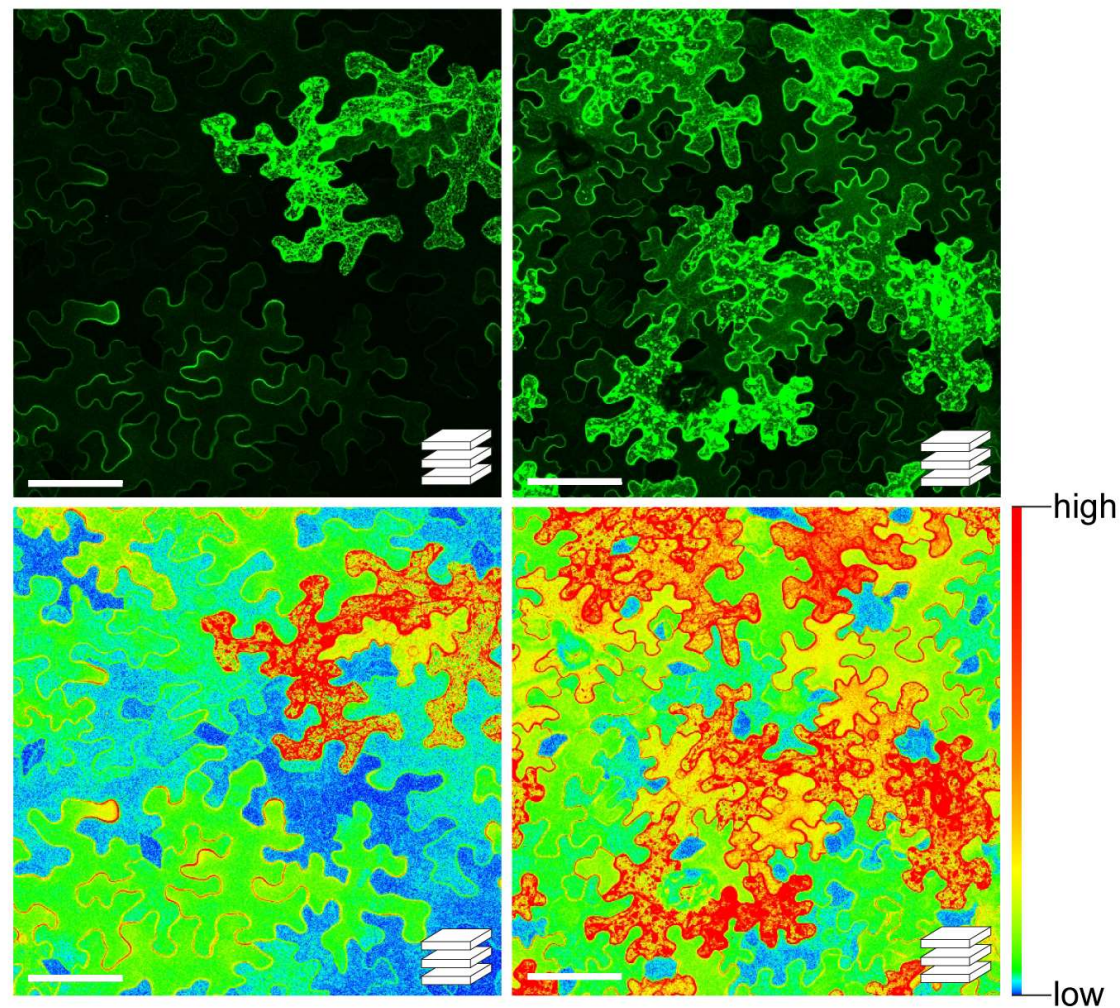

Figure 18: Localization of CRK7-mCitrine and CRK7-4-mCitrine transiently expressed in $N$. benthamiana.

A. tumefaciens cultures expressing CRK7-mCitrine or crk7-4-mCitrine under the control of native or $35 \mathrm{~S}$ promotor where infiltrated into $N$. benthamiana. Confocal microscopy (Leica SP5) was performed 2 dpi. Images are representative maximum projections (z-stack size: $50 \mu \mathrm{M}$ ). Scale bar $=100 \mu \mathrm{M}$. Lower panels show false color representations of fluorescence intensities, the intensity legend is shown on the right. 
3.1.5.2.2 Co-infiltration of $C R K 7-\mathrm{mCitrine}$ and $c r k 7-4-\mathrm{mCitrine}$ with cellular markers in $N$.

\section{benthamiana}

To confirm localization of CRK7-4-mCitrine to the ER and predominant CRK7-mCitrine fluorescence at the plasma membrane, both constructs were co-infiltrated with cellular markers in N. benthamiana. Confocal microscopy was performed two to three days post infiltration.

pUBQ10::mKate2-SYP122 was used as a plasma membrane marker (E. Petutschnig, unpublished), p35S::ER-ck (Nelson et al., 2007) as an ER marker and pUBQ10::mKate2-N7 (H. Ghareeb, unpublished) as nuclear marker.

First, co-localization studies were performed using CRK7/ CRK7-4-mCitrine proteins expressed from the $C R K 7$ promoter. These analyses focused on cells with low to moderate expression levels. The CRK7-mCitrine signal perfectly overlapped with mKate2-SYP122 fluorescence (Figure 19A), providing further evidence for CRK7-mCitrine localization at the plasma membrane. CRK7-mCitrine signal did not overlap at all with ER-ck and mKate2-N7 fluorescence (Figure 19B and C).

CRK7-4-mCitrine perfectly co-localized with the ER marker (Figure 19B), confirming that CRK7-4-mCitrine is restricted to the ER. No overlap of CRK7-4-mCitrine with mKate2SYP122 or mKate2-N7 was observed, but these experiments confirmed CRK7-4-mCitrine fluorescence at the nuclear envelope (Figure 19A and C).

The same results were obtained for the p35S::CRK7-mCitrine and p35S::crk7-4-mCitrine constructs (Figure S3). 
A

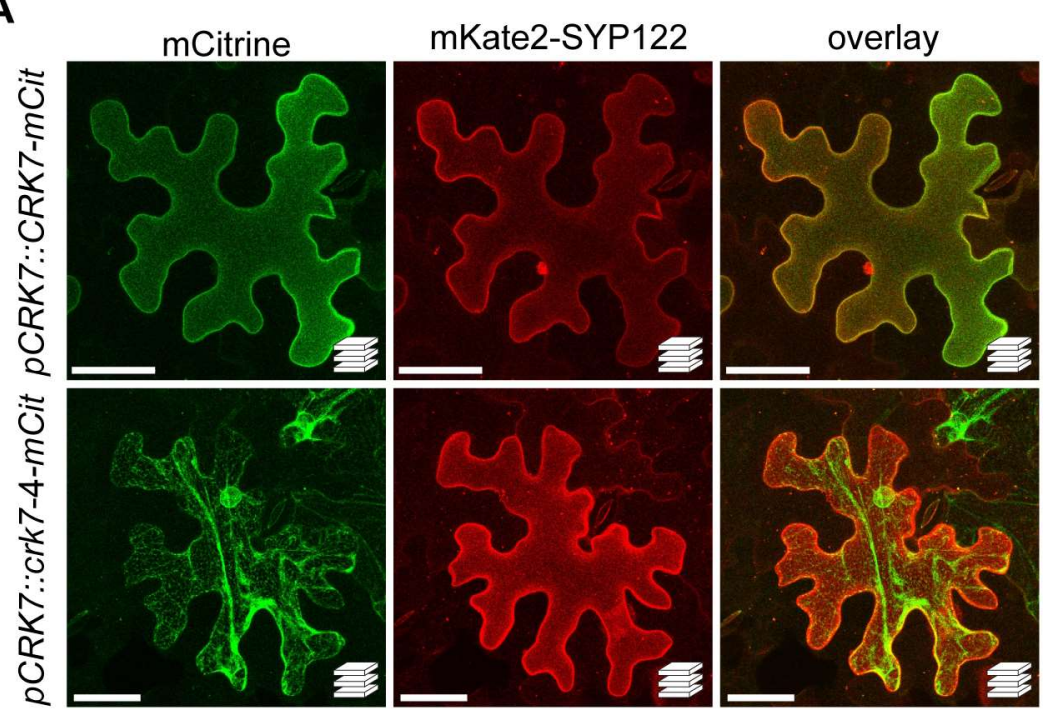

B

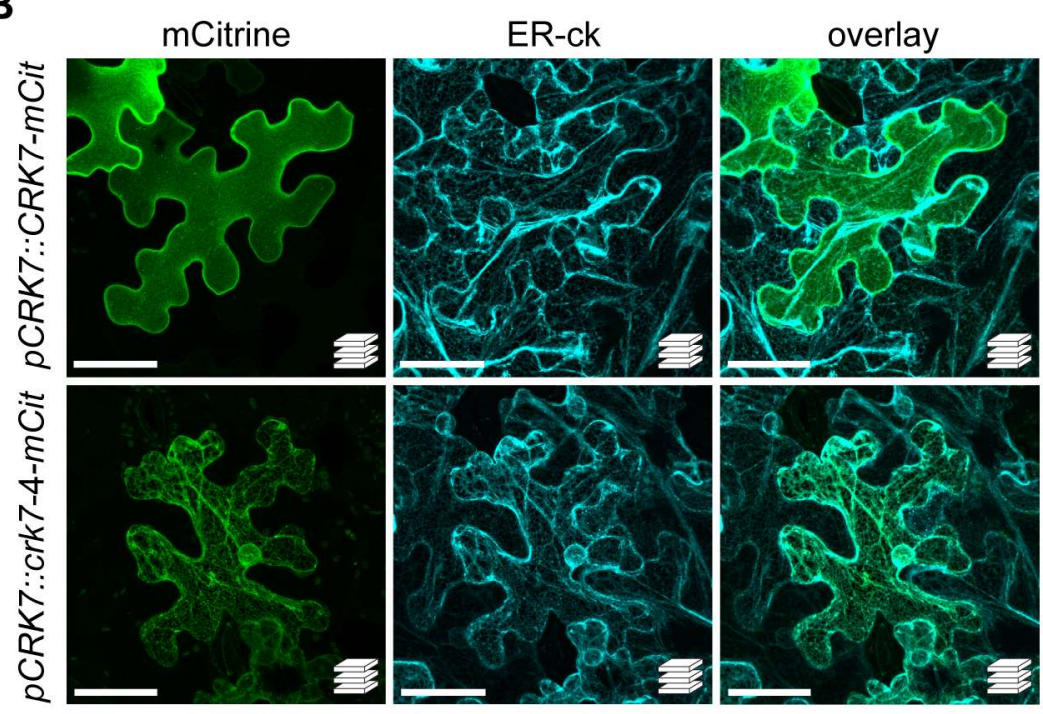

C

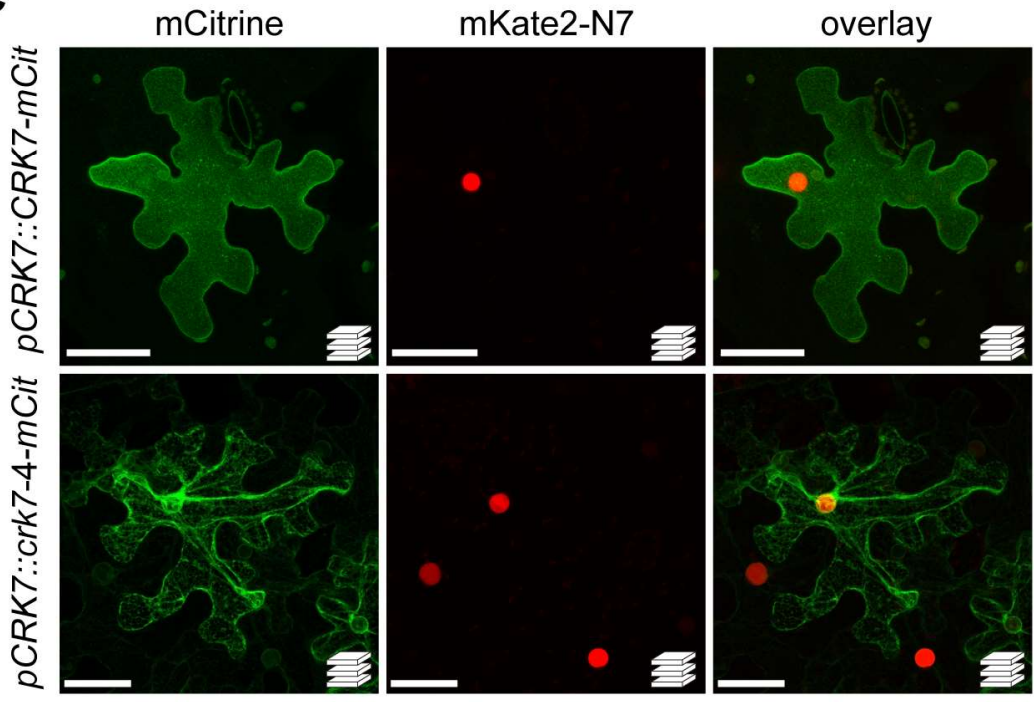

Figure 19: $C R K 7-\mathrm{mCit}$ and $c r k 7-4-\mathrm{mCit}$ co-infiltrated with cellular markers.

A. tumefaciens cultures transformed with $p$ CRK7::CRK7-mCitrine (mCit) or $p$ CRK7::crk7-4-mCit were mixed with cultures transformed with $p U B Q 10: \because$ mKate2-SYP122, p35S::ER-ck or pUBQ10::mKate2-N7 prior to infiltration into $N$. benthamiana. Confocal microscopy (Leica SP8) was performed 2-3 dpi. Imag- 
es are representative maximum projections. Scale bar $=50 \mu \mathrm{M}$. Green $=$ mCitrine. A: Co-infiltration with mKate2-SYP122 (red; E. Petutschnig, unpublished); Z-stack size 25-32 $\mu \mathrm{M}$. B: Co-infiltration with ERck (turquoise; Nelson et al., 2007); Z-stack size $19 \mu \mathrm{M}$. C: Co-infiltration with mKate2-N7 (red, H. Ghareeb, unpublished); Z-stack size $19 \mu \mathrm{M}$.

3.1.5.2.3 CRK7-mCitrine and CRK7-4-mCitrine subcellular localization in stably transformed Arabidopsis

CRK7-mCitrine was shown to localize to the plant plasma membrane after transient expression in N. benthamiana (3.1.5.2.2). To examine if this holds true also in Arabidopsis, CRK7$m$ Citrine and crk7-4-mCitrine under both the native and $35 S$ promotor were transformed into Col-3 gll via Agrobacterium mediated floral dip. Uninfected T1 plants were analyzed via confocal microscopy.

19 plants transformed with $p C R K 7:: C R K 7-m$ Citrine and the same number of plants transformed with $p C R K 7:: c r k 7-4-m$ Citrine were analyzed but in none of them any fluorescence was detectable, similar to the observations for $p C R K 7:: C R K 7 /$ crk7-4-Citrine expressing Arabidopsis plants (3.1.5.1.2; Data not shown).

19 T1 plants transformed with p35S::CRK7-mCitrine were analyzed. Eight of them did not show any fluoresce at all. In seven lines CRK7-mCitrine localized exclusively to the plasma membrane (Figure 20A). In two lines additionally to the plasma membrane signal, cytoplasmic strands and/ or a light ER-signal were detectable in a few cells (data not shown). Two other lines showed very high fluorescence in individual cells, resulting in ring-shaped structures and an overall granular microscopy signal (Figure 20B). These cells may have been undergoing cell death.

Out of 19 Col-3 gll plants transformed with p35S::crk7-4-mCitrine, 13 did not show fluorescence at all. In the other six T1 plants a microscopy signal was detectable in the ER (Figure 20C). 
A p35S::CRK7-mCit in Col-3 gl1
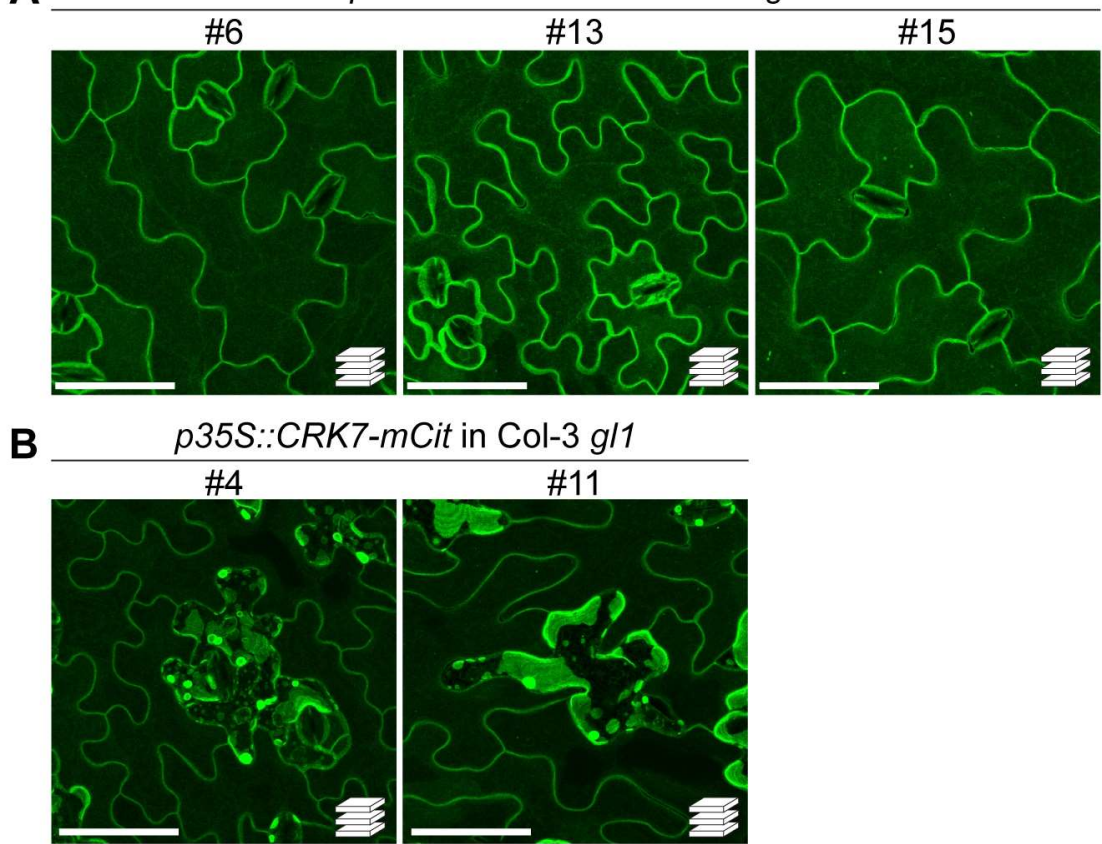

C p35S::crk7-4-mCit in Col-3 gl1
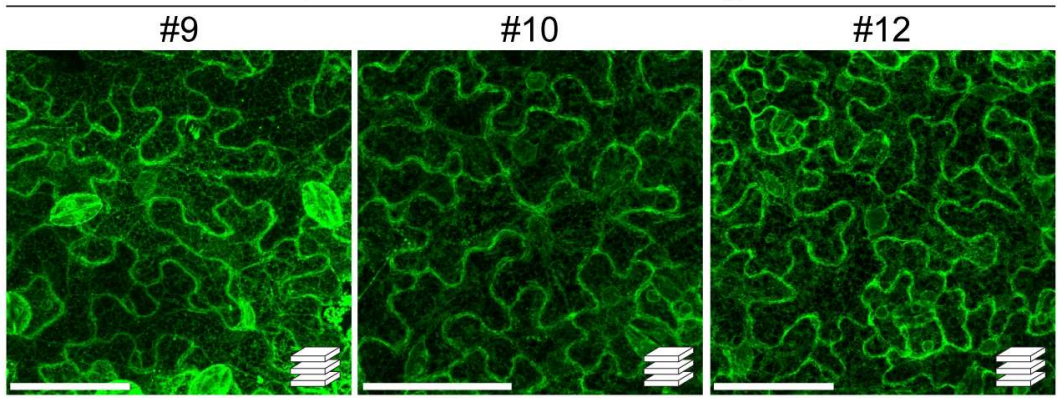

Figure 20: Localization of CRK7-mCitrine and CRK7-4-mCitrine in Arabidopsis.

Confocal microscopy (Leica SP8) was performed with uninfected four-week old stable transformed Arabidopsis $\mathrm{T} 1$ plants expressing CRK7-mCitrine (mCit) (A and B) or CRK7-4-mCit (C). Images are representative maximum projections of three (A, C) or two (B) independent lines (z-stack size: 15-20 $\mu \mathrm{M}$ ). Scale bar $=50 \mu \mathrm{M}$.

\subsubsection{Analysis of CRK7 and CRK7-4 protein function}

To get insights into the mechanism how CRK7 is involved in cell death induction, CRK7 and CRK 7-4 were analyzed via immunoblotting and co-immunoprecipitation experiments were performed. To identify possible interaction partners as well as phosphorylation sites in the proteins, proteomics experiments were conducted. In vitro kinase experiments were performed to investigate kinase activity of CRK7 and different CRK7 mutant versions. 


\subsubsection{A high molecular weight band is present in CRK7 Western Blots}

To characterize CRK7 and CRK7-4, proteins were extracted from $N$. benthamiana plants transiently expressing CRK7 or CRK7-4 fused to different tags under the native or $35 \mathrm{~S}$ promotor. Protein extracts from non-infiltrated $N$. benthamiana leaves were used as negative control. Total protein extracts were analyzed by SDS-PAGE (2.2.4.4) and Western Blot (2.2.4.5) using an $\alpha$-GFP antibody (Figure 21A, C and D).

Additionally, stably transformed Arabidopsis plants expressing CRK7-Citrine or CRK7-4Citrine under the native promotor were investigated in the T1 generation (Figure 21B).

The predicted molecular mass is $74 \mathrm{kDa}$ for CRK 7 and $27 \mathrm{kDa}$ for the Citrine tag. Therefore, non-modified CRK7-Citrine and CRK7-4-Citrine fusion proteins were expected to display an apparent mass of approximately $101 \mathrm{kDa}$. However, both CRK7-Citrine and CRK7-4-Citrine bands were detected around $120 \mathrm{kDa}$ (Figure 21A). The online tool NetNGlyc (Gupta et al., 2004) predicts six N-glycosylation sites in the CRK7 extracellular domain, potentially explaining the larger apparent mass. An additional band of high molecular weight ( $250 \mathrm{kDa})$ was detected in CRK7-Citrine immunoblots (Figure 21A). This band was quite strong in CRK7-Citrine samples, but much weaker in CRK7-4-Citrine lanes, suggesting that CRK7 forms very stable dimers or complexes either with itself or other proteins and this function appears to be strongly impaired in CRK7-4. Fragments running lower than $101 \mathrm{kDa}$ are likely CRK7-/ CRK7-4-Citrine degradation products.

The expression levels of CRK7-/ CRK7-4-Citrine from the CRK7 promoter in stably transformed Arabidopsis plants was lower than in transiently transformed N. benthamiana plants. Therefore an $\alpha$-GFP pull-down was performed for the Arabidopsis samples prior to Western Blot analysis. The high molecular weight band is present in CRK7-Citrine, but absent in CRK7-4-Citrine samples, confirming the results from N. benthamiana (Figure 21B). Additionally this shows that the aggregate formation is independent from protein expression levels.

The experiment was also performed with $N$. benthamiana plants expressing CRK7-mCitrine and CRK7-4-mCitrine to investigate if the tag plays a role for the formation of the high molecular weight band. Citrine can form dimers, whereas mCitrine is unable to dimerize (Cranfill et al., 2016). The high molecular weight band was also present in CRK7-mCitrine samples (Figure 21C), confirming this as an intrinsic CRK7 function that is independent from Citrine-dimerization. Additionally CRK7-FLAG and CRK7-4-FLAG were expressed in $N$. benthamiana. CRK7-FLAG has a predicted size of $77 \mathrm{kDa}$ but as observed for CRK7- 
(m)Citrine, also CRK7-FLAG and CRK7-4-FLAG ran higher than expected at around 100 $\mathrm{kDa}$ (Figure 21D). Three additional bands migrating at higher molecular weights ( $280 \mathrm{kDa}$, $\sim 215 \mathrm{kDa}, \sim 200 \mathrm{kDa}$ ) were detected in CRK7-FLAG samples, but absent in CRK7-4-FLAG samples (Figure 21D). Together, these data may suggest that CRK7 can form different homoor heteromers.

A

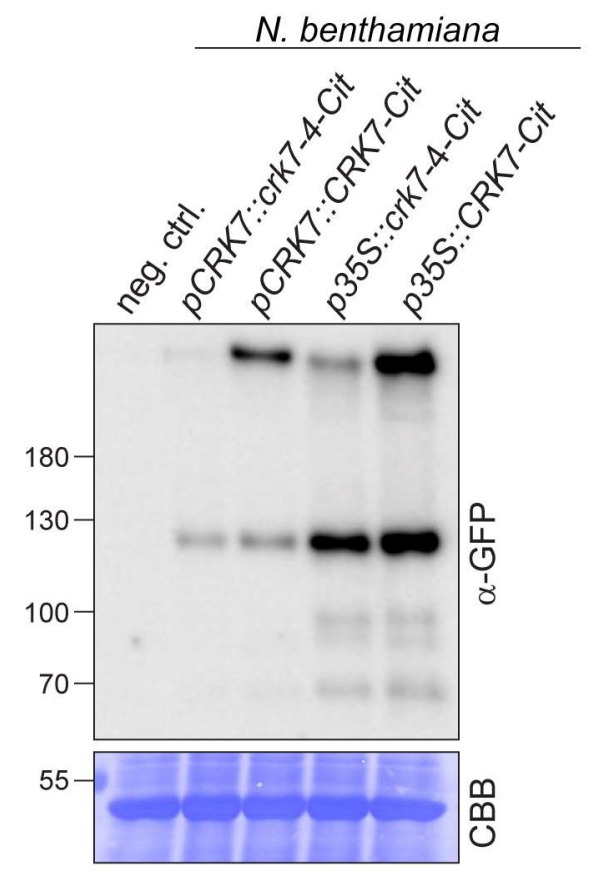

C

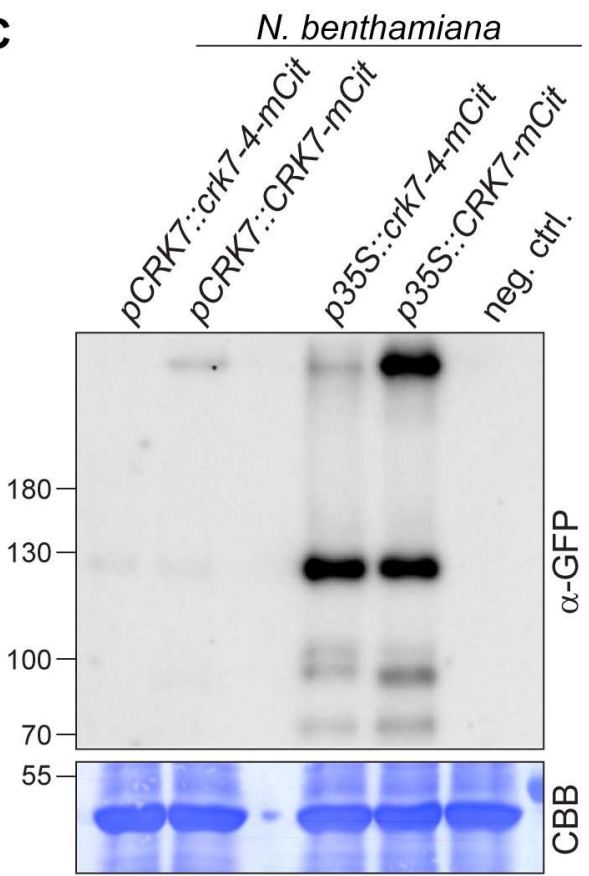

B

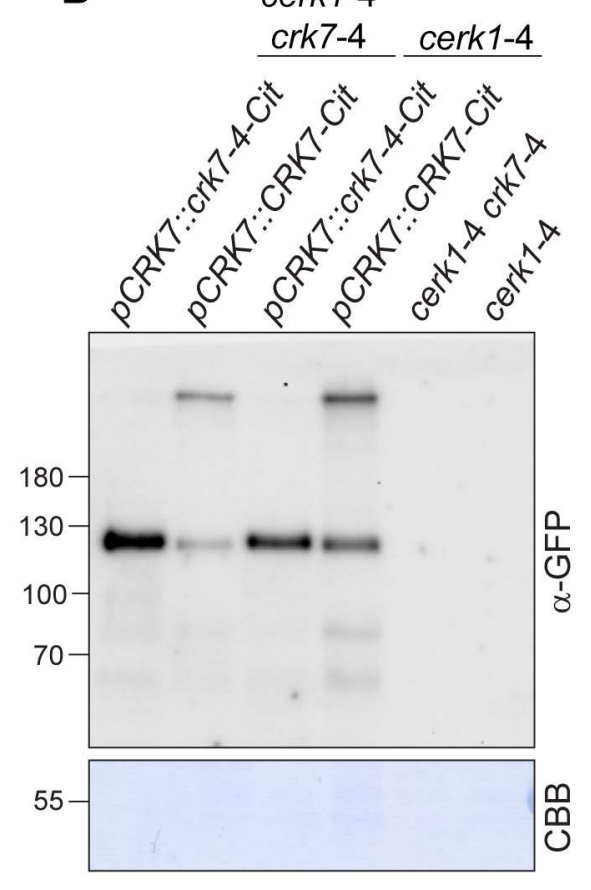

D

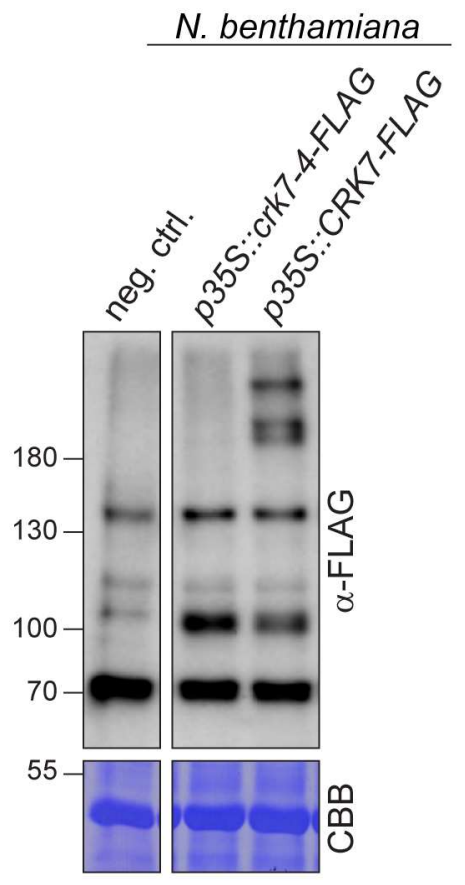




\begin{abstract}
Figure 21: Tagged CRK7 proteins show a high molecular weight band in Western Blot analysis.
A, C and D: Total proteins were extracted from transiently transformed $N$. benthamiana leaves and analyzed by SDS-PAGE and Western Blot using an $\alpha$-GFP antibody. Neg. ctrl. (Negative control)= Total protein extract of non-infiltrated $N$. benthamiana leaves. B: $p C R K 7: \because C R K 7-/ C R K 7-4-C i t r i n e$ (Cit) were stably expressed in Arabidopsis. $\alpha$-GFP pull-downs were used for immuno blot. Untransformed cerk1-4 and cerk1-4 crk7-4 plants served as negative controls. A, B and C: CRK7-(m)Cit and CRK7-4-(m)Cit migrate higher than the expected size of $101 \mathrm{kDa}$. Additionally to the monomeric CRK7/CRK7-4-(m)Cit band at about $120 \mathrm{kDa}$, a high molecular weight band is present. This fragment is more abundant in CRK7-(m)Cit than in CRK7-4-(m)Cit samples. B and C were performed by L. Pierdzig during Bachelor thesis (B) or Master laboratory rotation (C) supervised by C. Trippel. D: CRK7-FLAG and CRK7-4FLAG migrate higher than the expected size of $77 \mathrm{kDa}$. Additionally to the monomeric CRK7/CRK7-4FLAG band at about $100 \mathrm{kDa}$, three higher molecular weight bands are present only in CRK7-FLAG. $\mathrm{CBB}=$ Coomassie Brilliant Blue stained membrane.
\end{abstract}

\title{
3.1.6.2 CRK7 interacts with CRK7 whereas CRK7-4 is unable to interact with CRK7-4
}

CRK7 shows an additional band of very high molecular weight in Western Blot analysis that was much weaker in CRK7-4 (3.1.6.1). To investigate if CRK7 can form homodimers or oligomers, CRK7-mCitrine was co-expressed with CRK7-FLAG under both the native and $35 \mathrm{~S}$ promotor in $N$. benthamiana. In the same experiment CRK7-4-mCitrine was co-expressed with CRK7-4-FLAG under native and $35 S$ promotor. All constructs used for co-infiltration were also infiltrated separately as controls. Non-infiltrated $N$. benthamiana leaves were used as negative control. Expression of the mCitrine constructs was confirmed via confocal microscopy and infiltrated leaf areas were harvested 2.5 days post infiltration. Protein extracts were used for GFP pull-downs as well as for FLAG pull-downs (2.2.4.3.1). SDS-PAGE and Western Blots were performed using an $\alpha$-FLAG antibody (Figure 22A) and an $\alpha$-GFP antibody (Figure 22B) on total extracts, FLAG and GFP pull-downs.

As expected, CRK7 and CRK7-4 expression was stronger in $p 35 S$ than in $p C R K 7$ samples. The $\alpha$-GFP Western Blot performed on the GFP pull-down samples and the $\alpha$-FLAG Western Blot performed on the FLAG pull-down samples confirmed successful enrichment of the different fusion proteins (Figure 22).

Using the $\alpha$-FLAG antibody on the GFP pull-down samples revealed that CRK7-FLAG was pulled down together with CRK7-mCitrine (Figure 22A). This confirms that CRK7 is able to interact with itself and suggests that the high molecular weight band detected in Western Blots is caused by CRK7 homomerization (3.1.6.1). The observed CRK7-FLAG band in the GFP pull-down samples was considerably stronger upon expression under the $35 S$ promotor due to a general higher expression level, but a faint signal was also present upon expression under the native promotor (Figure 22A). For the co-infiltration of CRK7-4-FLAG with 
CRK7-4-mCitrine no signal was detectable with the $\alpha$-FLAG antibody after GFP pull-down suggesting that CRK7-4-FLAG and CRK7-4-mCitrine are unable to interact.

Vice versa, CRK7-mCitrine was detected in FLAG pull-down samples (Figure 22B). Because of the higher CRK7-mCitrine expression level in $p 35 S$ samples the observed band was stronger in $p 35 S$ than in $p C R K 7$ samples. For the co-infiltration of CRK7-4-mCitrine with CRK7-4-FLAG no signal was detectable with the $\alpha$-GFP antibody after FLAG pull-down confirming the result that CRK7-4-mCitrine and CRK7-4-FLAG cannot form homomers.

A

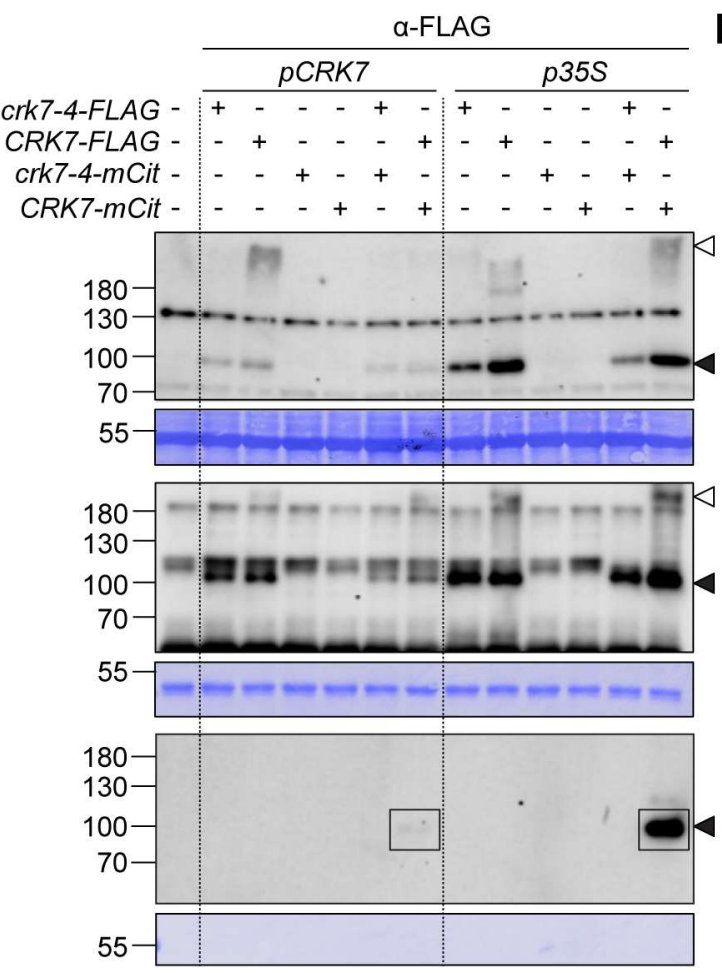

B

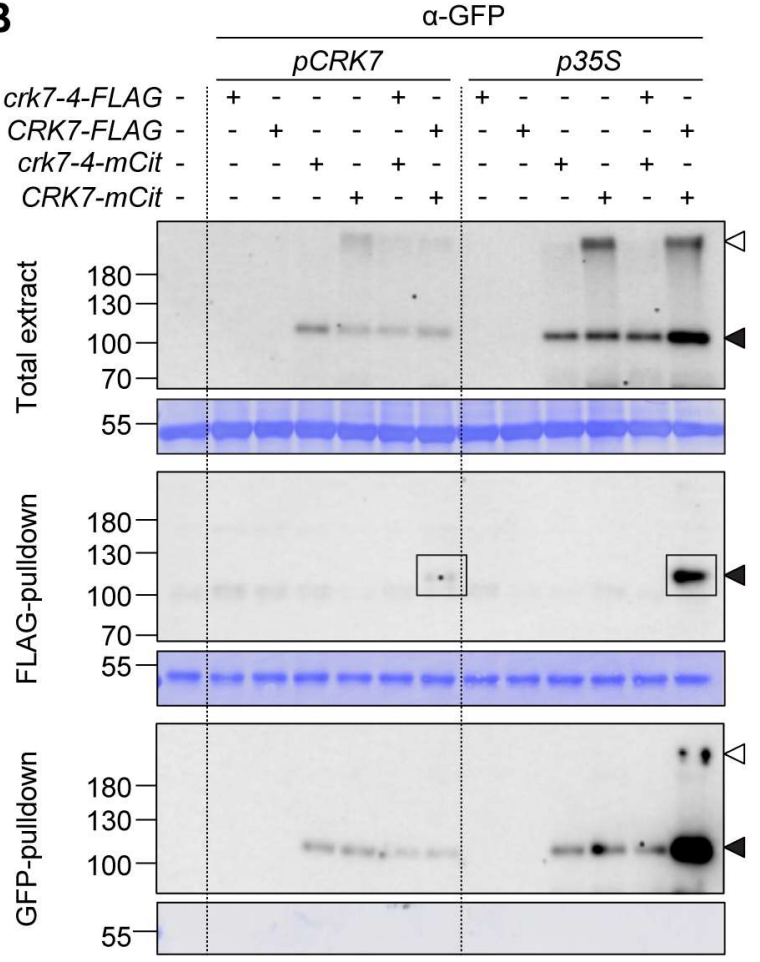

Figure 22: CRK7 is able to form homo- or multimers whereas CRK7-4 is not.

$C R K 7$-mCitrine (mCit) and CRK7-FLAG as well as crk7-4-mCit and crk7-4-FLAG were transiently coexpressed under both native and $35 \mathrm{~S}$ promoters in $N$. benthamiana. All constructs were additionally expressed individually. Non-infiltrated $N$. benthamiana leaves were used as negative controls. Protein extracts were used for GFP and FLAG pull-down. Western Blots on the total extracts (upper gels), the FLAG pull-downs (middle gels) and the GFP pull-downs (lower gels) were performed using the $\alpha$-FLAG antibody (A) and the $\alpha$-GFP antibody (B). Coomassie Brilliant Blue stained membranes confirming equal loading of the gels are shown below the Blots. Monomeric bands are indicated by filled arrowheads, high molecular bands by open arrowheads. Bands indicating CRK7 interaction are boxed.

\subsubsection{Proteomics experiments identified phosphorylation sites in CRK7 and CRK7-4}

To identify phosphorylated amino acids in CRK7 and CRK7-4, CRK7-mCitrine and CRK74-mCitrine were expressed in $N$. benthamiana under both the native and $35 S$ promotor. Expression was confirmed via confocal microscopy two days post infiltration and infiltrated leaf 
areas were harvested. Non-infiltrated $N$. benthamiana leaves were included as negative control. Affinity purification was performed with GFP-trap agarose beads followed by an onbead tryptic digestion and C18 purification (2.2.4.9.1). Purified samples were analyzed via

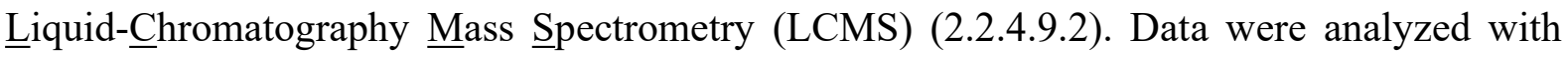
the Proteome Discoverer ${ }^{\mathrm{TM}}$ software using two different programs, Mascot and SEQUEST, to

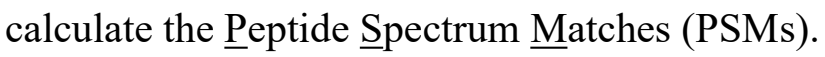

\subsection{CRK7 is highly phosphorylated in the kinase domain}

Initial proteomics experiments revealed that CRK7 is a highly phosphorylated protein. Many phosphopeptides matching the CRK7 juxtamembrane domain, the kinase domain and the Cterminus could be identified. CRK7-4 was overall much less phosphorylated than CRK7 with fewer distinct phosphopetides and much lower spectral counts. To investigate this in more detail, 20 highly abundant phosphopeptide ions (Figure 23A) were analyzed in a targeted selected ion monitoring (tSIM) approach (2.2.4.9.2) This method allows sensitive and quantitative detection of selected peptide ions and is therefore suitable for confirmation and quantification of protein phosphorylation (Borràs and Sabidó, 2017).

PSMs matching the selected phosphopeptide ions were counted and normalized to the total CRK7/ CRK7-4 PSMs. This was performed for CRK7-mCitrine and CRK7-4-mCitrine expressed under the native (Figure 23B) and $35 \mathrm{~S}$ promotor (Figure $23 \mathrm{C}$, Figure $\mathrm{S} 4$ ) in $N$. benthamiana plants. In all experiments, the total number of phosphopeptide PSMs identified in CRK7 was considerably higher than in CRK7-4 (Figure 23B and C; Figure S4). The highest number of phosphopeptide PSMs in CRK7 aligned to the activation (AL) and P+1 loop of the kinase domain. In CRK7-4 no (Figure 23B) or a considerably lower number (Figure 23C, Figure S4) of phospho-PSMs aligning to these regions were identified. The activation loop is a centrally located loop that is involved in switching the kinase activity on and off. Phosphorylation of the activation loop leads to large conformational changes that allow substrate binding and catalysis. The $\mathrm{P}+1$-loop interacts with the residue adjacent to the phosphorylated residue of the peptide substrate.

In contrast, for phosphopetides aligning to the C-terminus only minor differences in phorphorylation between CRK7 and CRK7-4 were observed. 


\begin{tabular}{|c|c|c|c|c|c|c|c|}
\hline \multirow{2}{*}{ No } & \multirow{2}{*}{ Sequence } & \multirow{2}{*}{ Charge } & \multicolumn{3}{|c|}{ Modifications } & \multirow{2}{*}{\multicolumn{2}{|c|}{ Localization in protein }} \\
\hline & & & $\mathrm{P}$ & 0 & C & & \\
\hline 1 & KTYGTTPALDEDDKTTIESLQLDYR & $3-4$ & 1 & & & luxtamem & rane domain \\
\hline 2 & TYGTTPALDEDDKTTIESLQLDYR & 3 & 1 & & & viniaimitim & 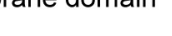 \\
\hline 3 & GGFGDVYKGTFSNGTEVAVKR & 3 & 1 & & & & $1+11$ \\
\hline 4 & IFGMDQTQQNTSR & 2 & 1 & $0-1$ & & & $\mathrm{AL}$ \\
\hline 5 & IFGMDQTQQNTSRIVGTYGYMSPEYAMR & 3 & $1-2$ & $0-3$ & & & VIII, AL, P+1 \\
\hline 6 & IVGTYGYMSPEYAMR & 2 & 1 & $0-2$ & & & VIII, $P+1$ \\
\hline 7 & SDVYSFGVLVLEIISGRKNNSFIETDDAQDLVTHAWR & 6 & 3 & & & domain & $\mathrm{IX}+\mathrm{X}$ \\
\hline 8 & NGTALDLVDPFIADSCR & 2 & 1 & & 1 & & $\mathrm{X}$ \\
\hline 9 & NGTALDLVDPFIADSCRK & $2-3$ & 1 & & 1 & & $x$ \\
\hline 10 & NGTALDLVDPFIADSCRKSEVVR & 3 & 1 & & $0-1$ & & $x$ \\
\hline 11 & CTHIGLLCVQEDPVKRPAMSTISVMLTSNTMALPAPQQPGFFVR & 4 & 1. & $0-1$ & 2. & & $\mathrm{XI}$ \\
\hline 12 & SRPGTNRLDSDQSTTNK & $2-3$ & $1-2$ & & & & \\
\hline 13 & SRPGTNRLDSDQSTTNKSVTVSIDDK & $3-4$ & $1-2$ & & & & \\
\hline 14 & LDSDQSTTNKSVTVSIDDK & 3 & 1 & & & C-terminus & \\
\hline 15 & LDSDQSTTNKSVTVSIDDKSMSDLDPR & 4 & 1 & & & & \\
\hline 16 & SVTVSIDDKSMSDLDPR & $2-3$ & 1 & 1 & & & \\
\hline
\end{tabular}

B

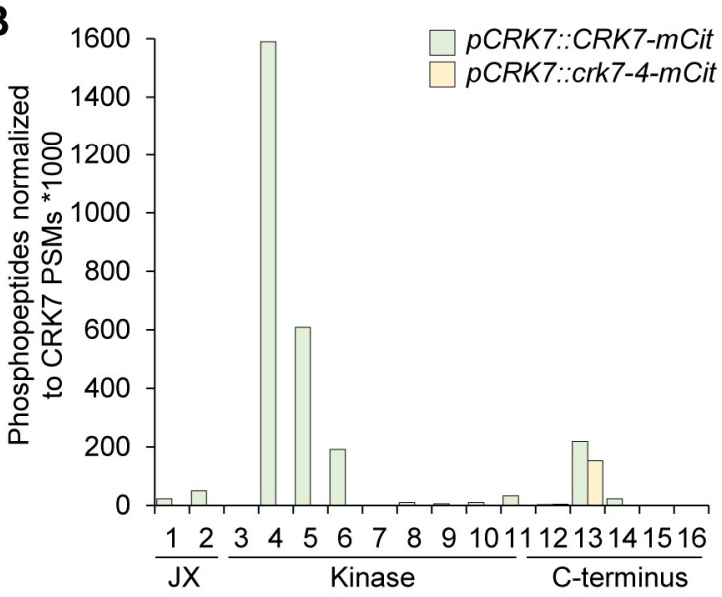

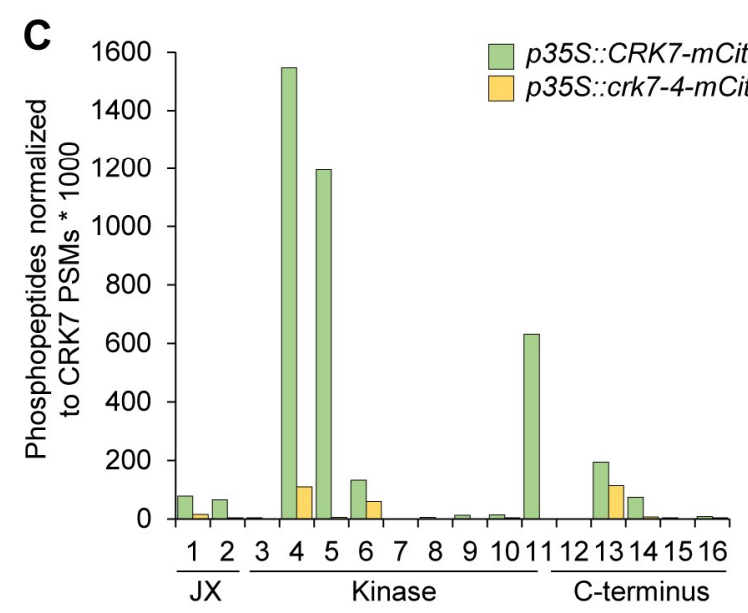

Figure 23: CRK7 shows higher levels of phosphorylation in the activation and P+1 loop than CRK74.

CRK7-mCitrine ( $m$ Cit) and crk7-4-mCit were transiently expressed in $N$. benthamiana under the native (B) or $35 S$ (C) promotor. Infiltrated leaf areas were harvested two dpi and proteins were extracted. An overnight $\alpha$-GFP pull-down was performed, followed by an on-bead tryptic digestion and a C18purification. The samples were used for liquid chromatography mass spectrometry (LC-MS) with the Orbitrap Q Exactive HF mass analyzer. A: Sequences and localization of the analyzed phosphopeptides are shown. 20 phosphopeptide ions were chosen for targeted selected ion monitoring (tSIM) analysis. Peptide ions with identical sequence but different charges or modifications were summarized. Numbers of modifications are displayed; $\mathrm{P}=$ phosphorylation, $\mathrm{O}=$ Oxidation, $\mathrm{C}=$ Carbamidomethylation. Roman numerals refer to kinase subdomains. $\mathrm{AL}=$ activation loop; $\mathrm{P}+1=\mathrm{P}+1$ loop. $\mathbf{B}+\mathbf{C}$ : Data show the number of identified peptide spectrum matches (PSMs) normalized to total CRK7 PSMs *1000. Numbers $1-16$ refer to the phosphopeptides in A. JX= Juxtamembrane domain.

To assign phosphorylation to specific amino acids within the phosphopeptides, the ptm $R S$ method within the Proteome Discoverer ${ }^{\mathrm{TM}}$ software was used. Often several phosphorylatable amino acids (tyrosine $(\mathrm{Y})$, serine $(\mathrm{S})$ and threonine $(\mathrm{T})$ ) are present in one peptide. Especially when these amino acids are located in close proximity within the peptide, unequivocal assignment may not be possible. ptmRS gives probabilities (0-100 \%) for all possible modifica- 
tion positions. For this experiment a cut-off was set to $95 \%$ probability for the ptm $R S$ data. This means that PSMs with ambiguous phosphorylation site assignment are not considered in this analysis. Assignment and quantification of phosphorylation sites for $p C R K 7: \because C R K 7 /$ crk7-4 and $p 35 S:: C R K 7 /$ crk7-4 samples are shown in figure 24 . The data for a further experiment with $p 35 S$-driven constructs are displayed in figure S5.

Amino acid residues that are specifically phosphorylated in the CRK7 juxtamembrane domain, but not in the CRK7-4 juxtamembrane domain were identified (Figure 24). The Cterminus of both CRK7 and CRK7-4 is also phosphorylated at different amino acids. This phosphorylation is tending to be stronger in CRK7 compared to CRK7-4. However, the greatest difference in phosphorylation is found in amino acids T491, T495 and T496 that belong to the activation loop as well as in S506 that is part of the $\mathrm{P}+1$-loop. These amino acid residues are highly phosphorylated in CRK7 but not at all or considerably less phosphorylated in CRK7-4 (Figure 24).

Proteomics experiments with CRK7-/ CRK7-4-Citrine after transient expression in N. benthamiana under the native and $35 S$ promotor (one replicate, table S1) and with Arabidopsis cerk1-4 T2 plants expressing CRK7-Citrine or CRK7-4-Citrine under the 35S promotor (one replicate, table $\mathrm{S} 2$ ), confirmed these findings. 


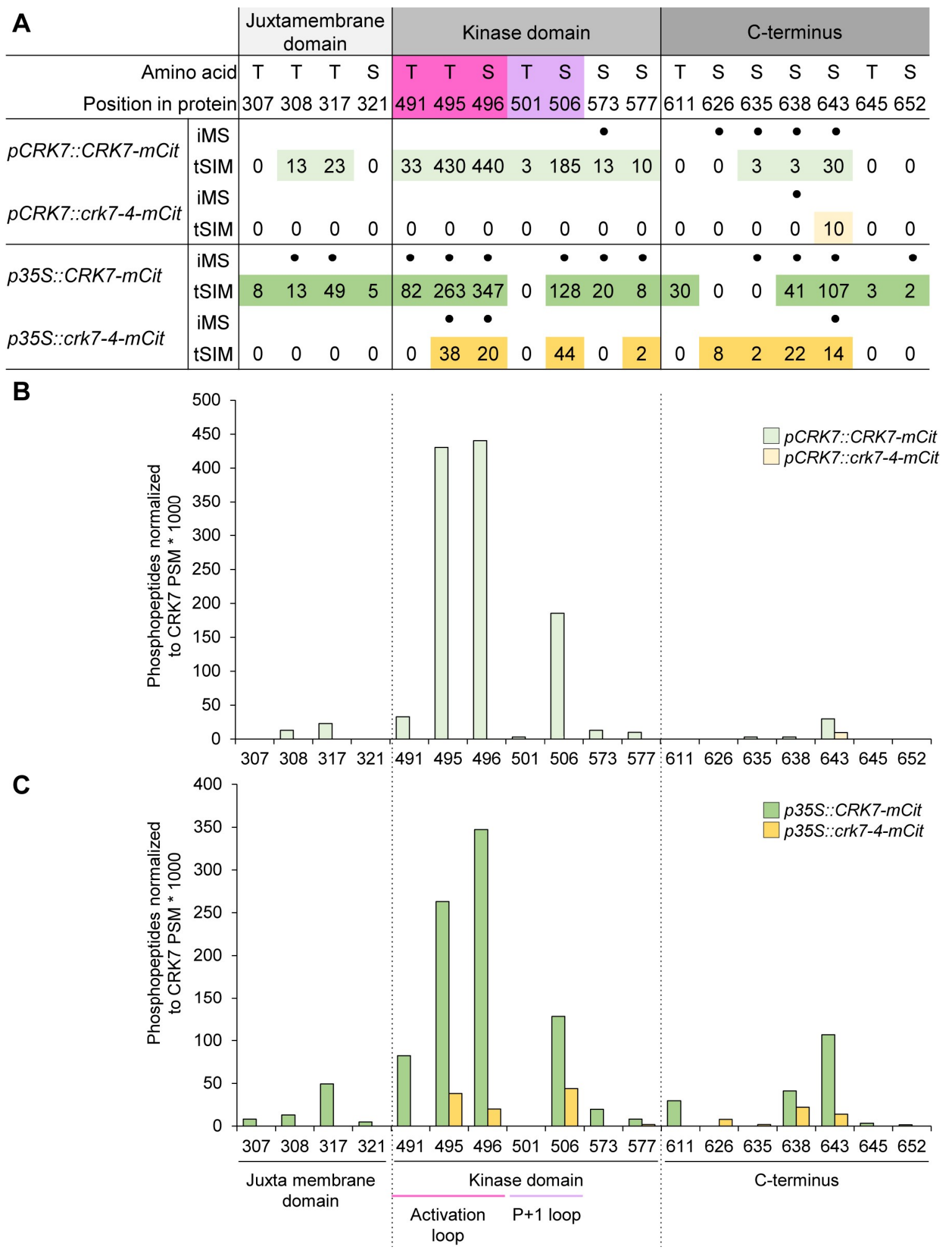

Figure 24: Phosphorylation sites identified in CRK7 and CRK7-4.

CRK7-mCitrine ( $m$ Cit) and crk7-4-mCit were transiently expressed in $N$. benthamiana under the native (B) or $35 \mathrm{~S}$ promotor (C). Infiltrated leaf areas were harvested 2 dpi and proteins were extracted. An overnight $\alpha$-GFP pull-down was performed, followed by an on-bead tryptic digestion and a C18-purification. The samples were used for LC-MS with the Orbitrap Q Exactive HF mass analyzer. 20 phosphopeptide ions were chosen for targeted selected ion monitoring (tSIM) analysis. Phosphorylation sites were identified with the ptmRS node. Counts for phosphorylation sites with a probability of $\geq 95 \%$, normalized to the total CRK7 PSMs, are displayed. A: Dots represent phosphorylation sites identified in the initial LCMS run (iMS). Numbers show counts of identified phosphorylation sites normalized to total CRK7 PSMs 
* 1000. Amino acids highlighted in pink belong to the activation loop, amino acids highlighted in purple to the $\mathrm{P}+1$ loop. $\mathbf{B}+\mathbf{C}$ : Phosphorylation sites identified in tSIM runs by $\mathrm{ptm} R S$ with a probability of $\geq 95$ $\%$ normalized to the total CRK7 PSMs * 1000 .

3.1.6.3.2 CRK7-4 interacts with ER chaperones involved in protein folding in N. benthamiana

Generated LC-MS data were analyzed to identify potential interaction partners of CRK7mCitrine and CRK7-4-mCitrine. The experiments were conducted with fusion proteins transiently expressed in $N$. benthamiana. Nevertheless, results could possibly give leads for interaction partners in Arabidopsis.

Interestingly, a number of calnexins (Figure 25A) and luminal-binding proteins (BiPs; Figure 25B) were identified to interact specifically with CRK7-4, but not or at a considerably lower level with CRK7. Calnexin monitors the efficient folding of glycoproteins in the ER and is a component of the ER quality control. ER quality control is necessary to ensure that only properly folded and assembled proteins proceed further along the secretory pathway. Luminal-binding proteins bind to proteins entering the ER to prevent their aggregation (Otero et al., 2010; Howell, 2013 Braackman et al., 2013).

In CRK7-4 a conserved cysteine residue (3.1.1) is mutated that was shown to be important for protein structure in other DUF26 proteins (Vaattovaara et al., 2019). Therefore, CRK7-4 might be misfolded and might be recognized by the ER quality control system, leading to its retention in the ER.

Interaction of CRK7-4 with ER chaperones was also observed in a proteomics experiments with Arabidopsis cerk1-4 plants expressing CRK7-Citrine or crk7-4-Citrine (Figure S6). 
A

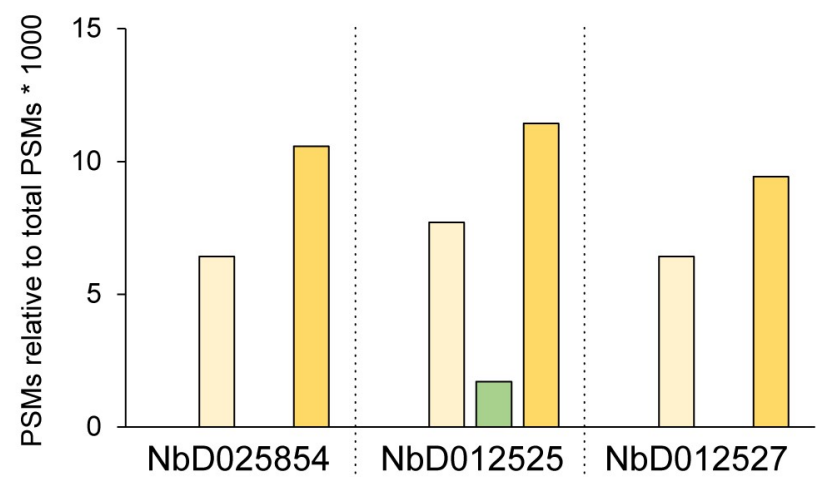

B

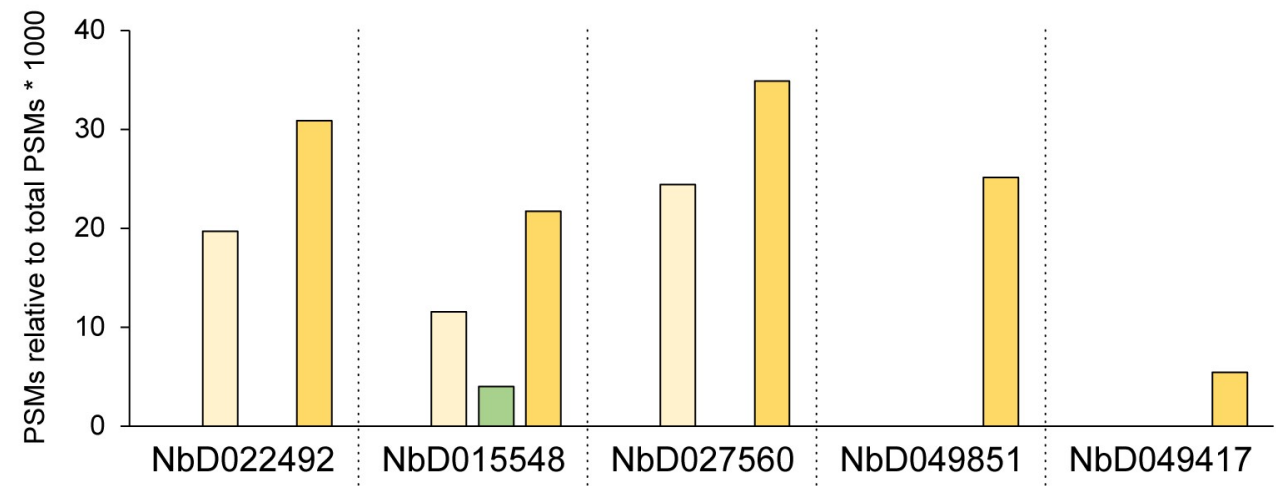

Figure 25: CRK7-4 interacts with ER chaperones in $N$. benthamiana.

CRK7-mCitrine ( $m$ Cit, green) and crk7-4-mCit (yellow) were transiently expressed in $N$. benthamiana under the native (lighter colors) or $35 \mathrm{~S}$ promotor (darker colors). Infiltrated leaf areas were harvested two dpi and proteins were extracted. An overnight $\alpha$-GFP pull-down was performed, followed by an on-bead tryptic digestion and a C18-purification. The samples were used for LC-MS with the Orbitrap Q Exactive HF mass analyzer. Peptide spectrum matches (PSMs) matching potential CRK7/ CRK7-4 interactors are shown: (A) calnexins and (B) luminal-binding proteins (BiPs). Only proteins with at least one protein unique peptide were considered. PSMs were normalized to total PSMs and multiplied with 1000 for better visualization.

3.1.6.4 CRK7 is an active kinase and phosphorylation of the activation and $\mathrm{P}+1$ loop is necessary for kinase activity

CRK7 possesses the conserved consensus motifs of active serine/ threonine protein kinases (Hanks et al., 1988). To investigate if CRK7 is an active kinase, Idänheimo et al. expressed the cytoplasmic domain of CRK7 as GST-tagged fusion protein in Escherichia coli. GSTCRK7 was able to phosphorylate the artificial substrate myelin basic protein (MBP) and showed autophosphorylation activity (Idänheimo et al., 2014).

CRK 7 expressed in $N$. benthamiana was shown to be highly phosphorylated in the activation and $\mathrm{P}+1$ loop and this phosphorylation was considerably lower in CRK7-4 (3.1.6.3.1). To confirm kinase activity of CRK7 and to investigate if the altered phosphorylation of the activation and $\mathrm{P}+1$-loop in CRK7-4 interferes with kinase activity, in vitro kinase activity experiments were conducted. 
Additionally, mutant CRK7 versions were included in the study in which the phosphorylated amino acids in either the activation loop $\left(\mathrm{CRK} 7_{\mathrm{ALmut}}\right)$ or in both the activation and $\mathrm{P}+1$ loop (CRK7 $7_{\mathrm{AL}+\mathrm{Pmut}}$ ), were mutated to non-phosphorylatable amino acids (Figure 26A).

CRK7-mCitrine, crk7-4-mCitrine, $C R K 7_{\mathrm{ALmut}} \mathrm{mCitrine}$ and $C R K 7_{\mathrm{AL}+\mathrm{Pmut}}-\mathrm{mCitrine}$ were transiently expressed in $N$. benthamiana under the $35 S$ promotor (2.2.1.2.2). Expression was confirmed two days post infiltration via confocal microscopy. The localization of CRK $7 \mathrm{ALmut}^{-}$ mCitrine and $\mathrm{CRK} 7 \mathrm{AL}+\mathrm{Pmut}-\mathrm{mCitrine}$ was comparable to CRK7-mCitrine (compare to 3.1.5; data not shown).

Infiltrated leaf areas were harvested two dpi and protein extracts were used for pull-down with GFP-trap agarose beads (2.2.4.3.2). Non-infiltrated leaves were included in the experiment as negative control.

Proteins were analyzed via SDS-PAGE on an $8 \%$ gel to investigate autophosphorylation and on a $15 \%$ gel to investigate MBP phosphorylation (2.2.4.6). Western Blot experiments using the $\alpha$-GFP antibody revealed that the samples express an uneven amount of protein (Figure 26D). This is probably due to transient expression in N. benthamiana, which often leads to patchy areas with distinct protein abundance levels within the infiltration zone.

In the autoradiogram of the $8 \%$ gel no signal was detectable in the negative control. Clear autophosphorylation activity was detected for CRK7-mCitrine, as well as for CRK7-4-

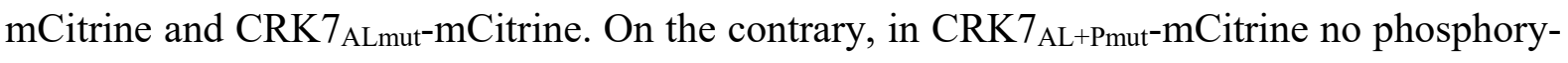
lation signal was detectable (Figure 26B, Figure S7). Due to the different protein amounts observed in Western Blot, band intensities of both the autoradiogram and the Western Blot were quantified and the autoradiogram band intensity was normalized to the Western Blot band intensity for every sample (Figure 26E). The data show that CRK $7_{\mathrm{AL}+\mathrm{Pmut}}-\mathrm{mCitrine}$ is kinase dead whereas the other CRK7 versions are able to autophosphorylate. In a second replicate (Figure $\mathrm{S} 7$ ) the result for $\mathrm{CRK} 7_{\mathrm{AL}+\mathrm{Pmut}} \mathrm{mCitrine}$ was confirmed, but also crk7-4mCitrine and $\mathrm{CRK} 7_{\mathrm{ALmut}}-\mathrm{mCitrine}$ showed a reduced autophosphorylation activity compared to CRK7-mCitrine. 
A

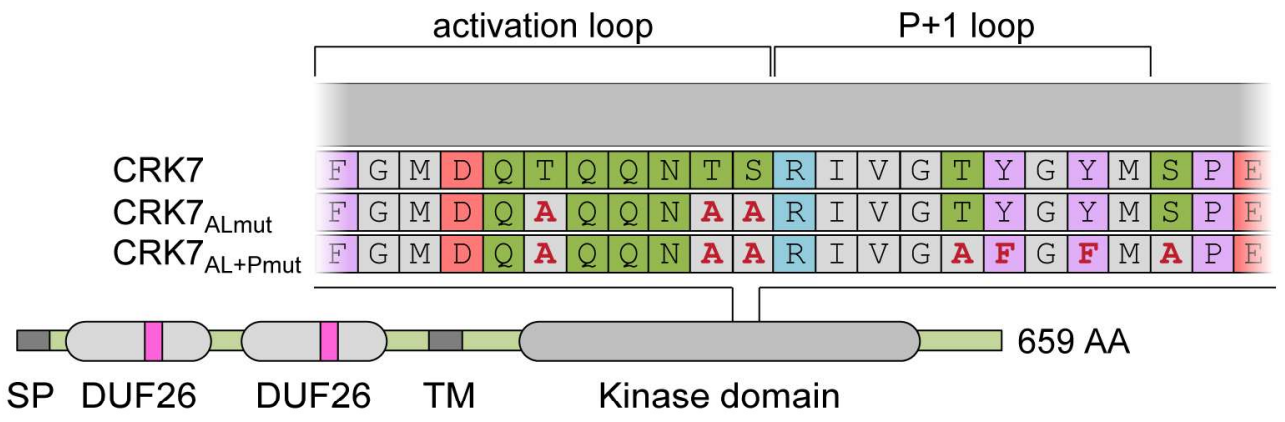

B

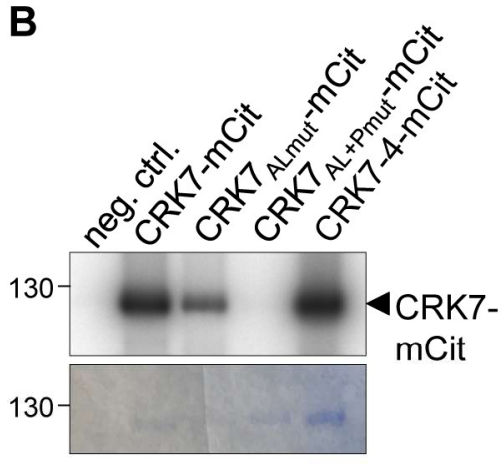

$8 \%$ gel

$E_{\text {o }}$

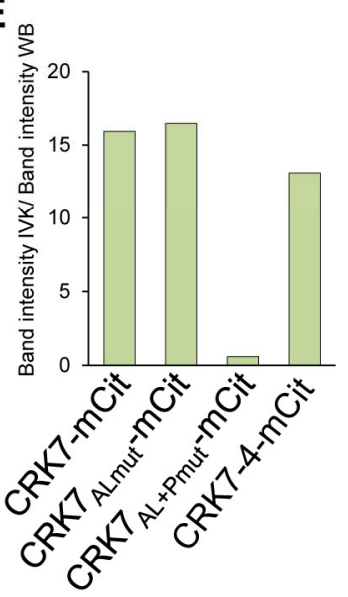

C

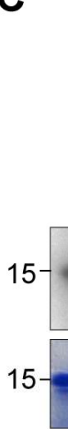

15

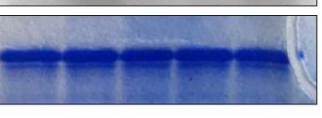

$15 \%$ gel

F

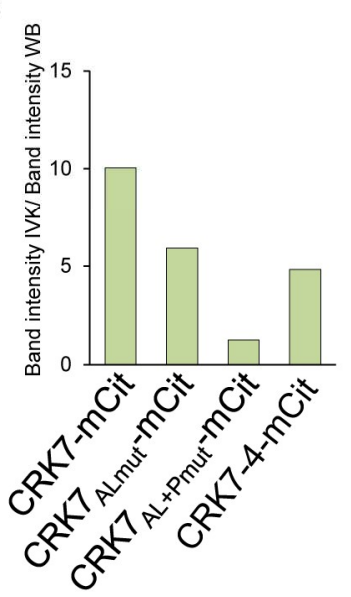

D

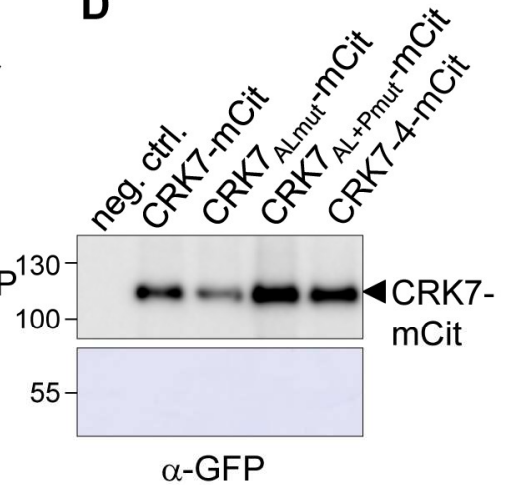

$\alpha-G F P$

Figure 26: CRK7 is an active kinase whereas $\mathrm{CRK} 7_{\mathrm{AL}+\mathrm{Pmut}}$ is kinase dead.

A: Overview of the CRK7 mutants used in this experiment. Predicted protein features: Signal peptide (SP), DUF26 domains with the internal $\mathrm{C}-\mathrm{X}_{8}-\mathrm{C}-\mathrm{X}_{2}-\mathrm{C}$ motif (pink box), transmembrane domain (TM) and kinase domain. Domains were predicted using SMART (http://smart.embl-heidelberg.de/, Letunic and Bork, 2018). A section of the CRK7 protein is shown in detail and mutations in CRK $7_{\mathrm{ALmut}}$ and $\mathrm{CRK} 7_{\mathrm{AL}+\mathrm{Pmut}}$ are marked in red. Color code for amino acids: Polar positive $=$ blue, polar negative $=$ red, polar neutral $=$ green, non-polar aliphatic $=$ grey, non-polar aromatic $=$ purple. $\mathbf{B}-\mathbf{F}:$ CRK7-mCit, CRK7 ${ }_{\mathrm{ALmut}}-\mathrm{mCit}, \mathrm{CRK} 7_{\mathrm{AL}+\mathrm{Pmut}} \mathrm{mCit}$ and $\mathrm{CRK} 7-4-\mathrm{mCit}$ were transiently expressed in $N$. benthamiana under the $35 \mathrm{~S}$ promotor. Expression was confirmed via CLSM and infiltrated leaf areas were harvested two dpi. Non-infiltrated leaves were used as negative control (neg. ctrl). Adjusted total protein extracts were used for an overnight GFP pull-down. CRK7 autophosphorylation (B) and phosphorylation of the artificial substrate myelin basic protein (MBP) (C) were tested. Coomassie stained gels are shown as loading controls. D: Western Blot of GFP pull-downs with the $\alpha$-GFP antibody. E + F: Quantification of the autoradiography band intensities of the $8 \%$ (E) and $15 \%$ gel (F) normalized to the Western Blot band intensities. Intensities of the negative control in the audiograms were subtracted from the respective samples prior to normalization. 
In the autoradiogram of the $15 \%$ gel a clear signal was detectable in the negative control (Figure 26C). This suggests that some $N$. benthamiana kinases that can phosphorylate MBP were pulled down with the GFP agarose trap. Nevertheless, MBP phosphorylation observed in the autoradiogram of the CRK7-mCitrine sample was considerably stronger than in the negative control (Figure 26C). This confirms that CRK7-mCitrine is able to phosphorylate the artificial substrate MBP (Idänheimo et al., 2014). The signal observed in the autoradiogram for both $\mathrm{CRK} 7_{\mathrm{ALmut}}-\mathrm{mCitrine}$ and $\mathrm{CRK} 7_{\mathrm{AL}+\mathrm{Pmut}}-\mathrm{mCitrine}$ were comparable to the signal in the negative control. CRK7-4-mCitrine shows a stronger MBP phosphorylation than observed in the negative control. An equal MBP amount was present in all samples as confirmed in the Coomassie Brilliant Blue stained gel (Figure 26C). Band intensities of the autoradiogram of the $15 \%$ gel were quantified and normalized to the Western Blot band intensities (Figure 26F, Figure S7). Even though the results on MPB phosphorylation are preliminary, they confirm that $\mathrm{CRK} 7_{\mathrm{AL}+\mathrm{Pmut}}$ is kinase dead. Furthermore, they suggest that CRK 7 ALmut as well as CRK7-4 might have reduced trans-phosphorylation abilities.

\subsection{CRK43}

3.2.1 Mapping by sequencing revealed a splicing mutation in CRK43 as possible cerk1-4 suppressor mutation

To identify further components in cerk $1-4$ cell death signaling, the forward genetic screen that yielded $c r k 7$ and other suppressor mutants was continued.

The M2 line noce8-1 was identified as suppressor (E. Petutschnig), propagated to the M3 generation and backcrossed with cerk1-4. 158 F2 plants of this cross were infected with $E$. cruciferarum. 42 of these plants (26.6\%) showed the hypersensitive cerkl-4 cell death phenotype and 40 plants $(25.3 \%)$ showed a clear suppressor phenotype with strong fungal growth on the leaves. Similar to the results obtained for noce9-1 (3.1.1), 76 (48.1\%) of the noce8-1 x cerk1-4 F2 plants showed an intermediate phenotype with only mild cell death and no or just very weak fungal growth. Representative images of plants with suppressor, nonsuppressor and intermediate phenotypes are shown in figure 27. This result suggests that similar to noce9-1, the suppressor mutation in noce8-1 is semi-dominant. 


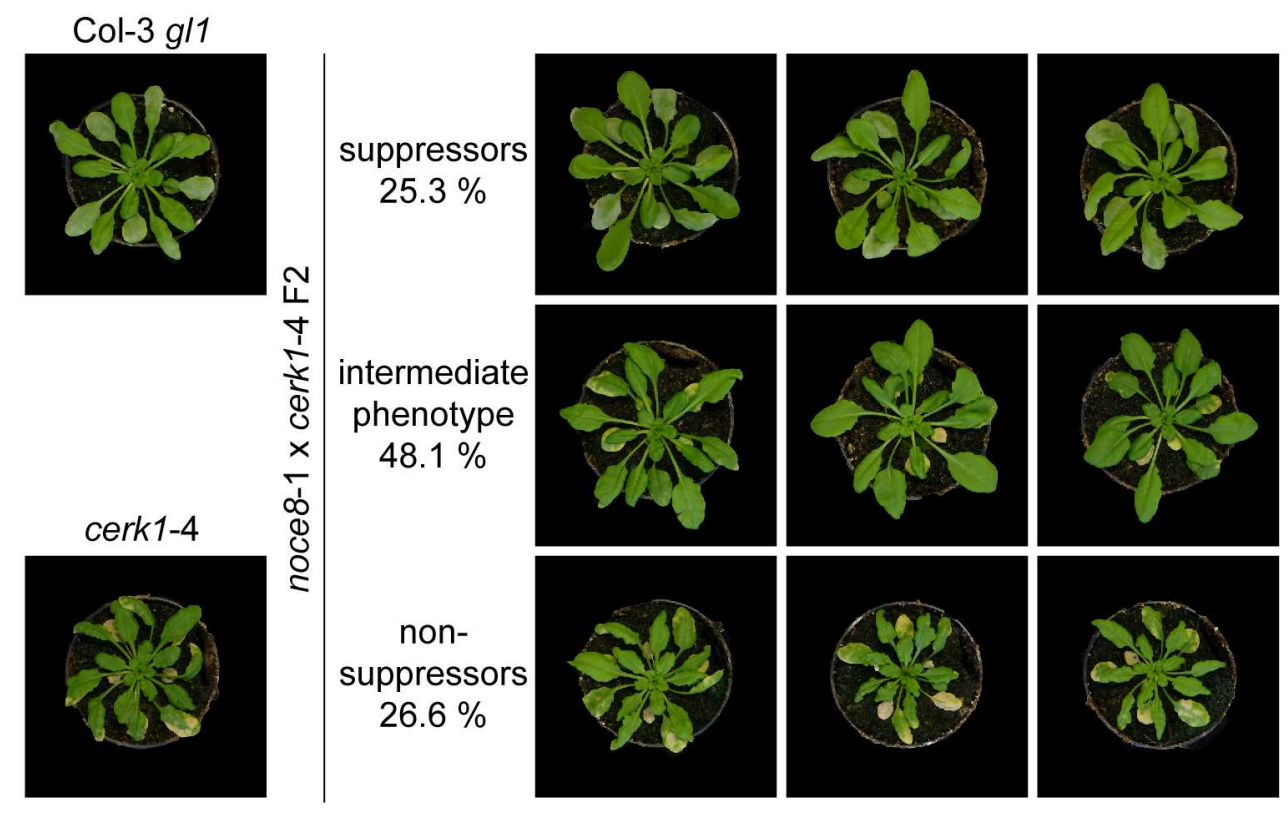

Figure 27: $E$. cruciferarum infected noce8-1 x cerk1-4 F2 plants show a semi-dominant suppressor phenotype.

Four-week old noce8-1 x cerk1-4 F2 plants and the according controls Col-3 gll and cerk1-4 were infected with E. cruciferarum. Pictures were taken 11 dpi. Plants were scored regarding cell death and fungal growth. Plants showing no cell death and strong fungal growth were considered as suppressors, plants with no fungal growth but strong cell death such as in the cerk1-4 control were classified as nonsuppressors and the remaining plants with no or a very low amount of fungal growth and mild cell death were classified as intermediate phenotype. Numbers below the categories show the percentage of plants with the according phenotype on the basis of 158 analyzed plants.

Leaves of all 40 suppressor plants were harvested, pooled and genomic DNA was extracted (2.2.3.1.2). The genomic DNA was used for $150 \mathrm{bp}$ paired-end Illumina sequencing and sequencing results were aligned to the Arabidopsis TAIR10 reference genome using CLC genomics workbench 10.1.1 (2.2.5.1). SNPs in noce8-1 were identified and SNPs that occurred in both the background line cerkl-4 and noce8-1 were removed. Moreover, SNPs identified in noce8-1 were compared to the sequencing data from three independent suppressor lines (M. Stolze, C. Meusel, E. Petutschnig, C. Trippel) and duplicates were removed in order to obtain noce8-1 specific SNPs. These were plotted against their chromosomal position (Figure 28). As explained for noce9-1 (3.1.1), the number and frequency of SNPs at the centromere is elevated due to alignment issues that are caused by a large number of repetitive sequences in this region (Round et al., 1997; Copenhaver et al., 1999). As only plants suppressing the cerkl-4 cell death were included in the pool for sequencing, the mutation causing this phenotype should be present in $100 \%$ of the reads. Unlinked SNPs have an expected SNP frequency of about $50 \%$. Genetically linked SNPs should show an elevated SNP frequency increasing with proximity to the causative mutation. This results in a SNP-frequency peak in the 
graph. For noce8-1 such a peak is visible only on chromosome one, suggesting that the mutation causing suppressor phenotype in noce8-1 is located on the lower arm of chromosome one (Figure 28).

Genes containing SNPs with frequencies of at least $90 \%$ were considered as suppressor candidates (Table 18, boxed in figure 28). The mutation with the highest frequency (98\%) was localized in the genomic region of cysteine-rich receptor-like protein kinase CRK43 (Atlg70740). Analysis of individual suppressor plants by PCR confirmed the genetic linkage of this mutation with the suppressor phenotype (Figure S8). Since CRK7 was identified as a molecular component necessary for cerkl-4 cell death induction (3.1) and since CRKs are highly conserved proteins, CRK43 was the most promising candidate for the suppressor mutation in noce8-1. This mutation was named crk43-2 (Figure 28). Among the 44 CRKs in Arabidopsis three CRKs are lacking the entire ectodomain and can be therefore considered as cysteine-rich receptor-like cytoplasmic kinases (CRCKs). CRK43 is one of these three CRCKs (Bourdais et al., 2015; Vaattovaara et al., 2019). Bioinformatic analyses using Myristoylator (Bologna et al., 2004), Aramemnon (Schwacke et al., 2003) and SMART (Letunic and Bork, 2018) were conducted to search for modifications possibly leading to plasma membrane localization, such as myristyolation. In CRK43 no myristyolation sites or other lipid modifications were predicted, suggesting cytoplasmic localization for CRK43. 

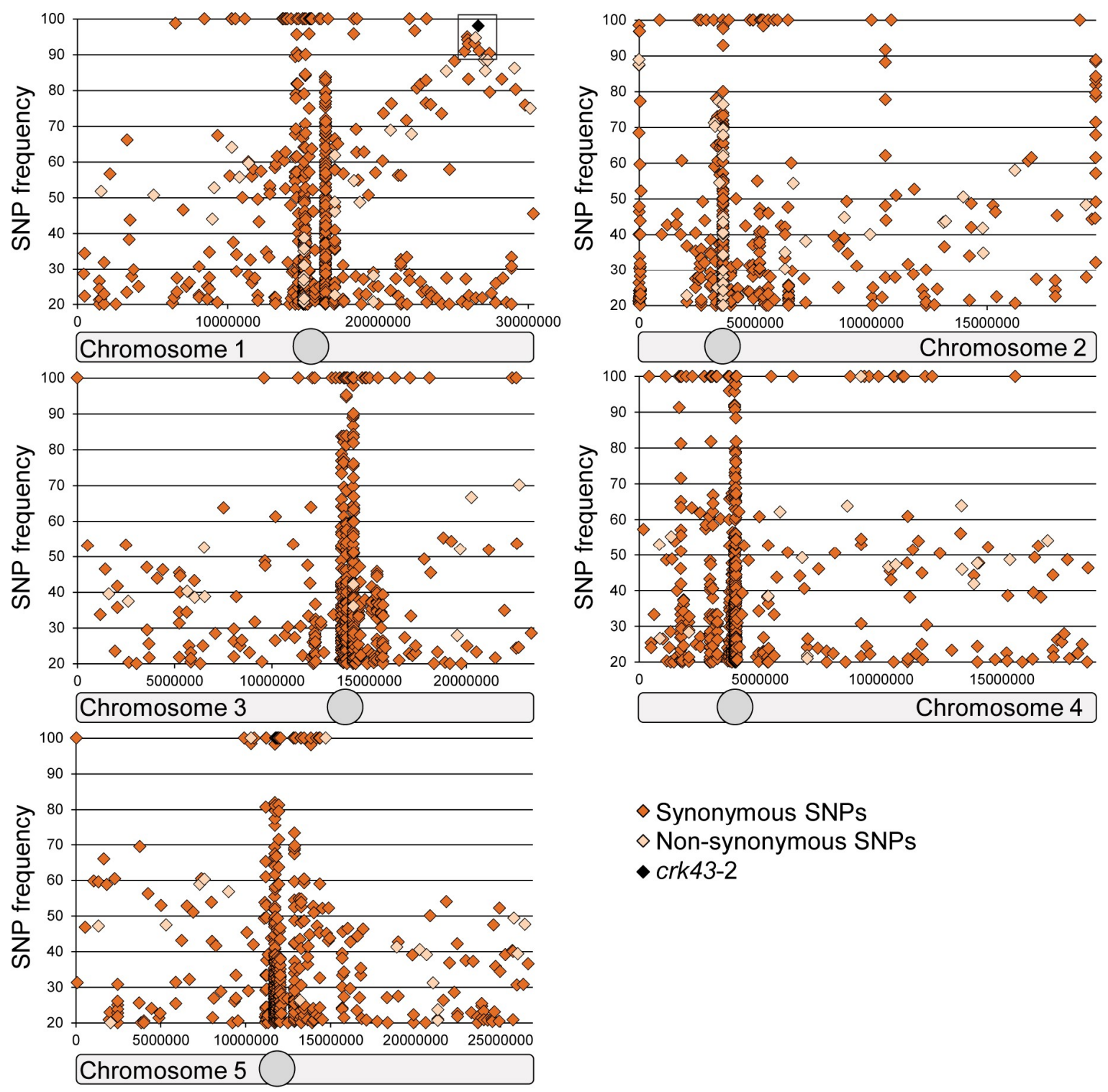

\section{$\diamond$ Synonymous SNPs \\ $\diamond$ Non-synonymous SNPs \\ - crk43-2}

\section{Figure 28: The suppressor mutation in noces-1 is located on the lower arm of chromosome one.}

$150 \mathrm{bp}$ paired end Illumina reads were aligned to the TAIR10 reference genome using CLC genomics workbench 10.1.1. noce8-1 suppressor SNP frequencies were mapped to their position on the chromosomes. Parameter for aligning and SNP call: Length fraction: 0.85; Similarity fraction: 0.8 ; minimum coverage $=10$, minimum counts $=2$, minimum frequency $=20 \%$; minimum central quality $=20 ;$ minimum neighborhood quality= 15. Duplicate SNPs present in cerk1-4, noce1-4, noce9-1 or noce12-23 were subtracted. Synonymous SNPs are shown as dark orange diamonds, SNPs causing non-synonymous mutations are depicted as light orange diamonds. Grey boxes below the graphs symbolize chromosomes with circles marking the centromeres. The black box highlights the region containing the suppressor mutation. The best candidate, crk43-2, is depicted as a black diamond. 
Table 18: Suppressor candidates in noce 8 -1.

\begin{tabular}{|c|c|c|c|c|c|c|}
\hline $\begin{array}{l}\text { Reference } \\
\text { position }\end{array}$ & SNP & Frequency & $\begin{array}{l}\text { Overlapping } \\
\text { annotations }\end{array}$ & $\begin{array}{l}\text { Coding region } \\
\text { change }\end{array}$ & $\begin{array}{l}\text { Amino acid } \\
\text { change }\end{array}$ & Annotation $^{c}$ \\
\hline 25948177 & $\begin{array}{l}\mathrm{C}>\mathrm{T} \\
\text { intron } 3\end{array}$ & 94.9 & At1g69030 & $662+210 \mathrm{G}>\mathrm{A}$ & & $\begin{array}{l}\text { BSD domain-containing } \\
\text { protein }\end{array}$ \\
\hline 26000791 & $\begin{array}{l}\mathrm{C}>\mathrm{T} \\
\text { exon } 1\end{array}$ & 93.0 & $\begin{array}{l}\text { At1g69160 } \\
\text { BGL1 }\end{array}$ & $477 \mathrm{C}>\mathrm{T}$ & & \\
\hline \multirow[t]{2}{*}{26502924} & $\begin{array}{l}\mathrm{C}>\mathrm{T} \\
\text { exon } 1\end{array}$ & 94.7 & $\begin{array}{l}\text { At1g70330 } \\
\text { ENT1 }\end{array}$ & $5 \mathrm{C}>\mathrm{T}$ & Thr2Ile & Adenosine transporter \\
\hline & $\begin{array}{l}\mathrm{C}>\mathrm{T} \\
\text { exon } 1\end{array}$ & & At1g70335 & $335 \mathrm{G}>\mathrm{A}$ & Ser112Asn & Hypothetical protein \\
\hline 26674933 & $\begin{array}{l}\mathrm{C}>\mathrm{T} \\
\text { intron } 3\end{array}$ & 98.0 & $\begin{array}{l}\text { At1g } 70740 \\
\text { CRK } 43\end{array}$ & $\begin{array}{l}446-1 \mathrm{G}>\mathrm{A}^{\mathrm{a}} \\
410-1 \mathrm{G}>\mathrm{A}^{\mathrm{b}}\end{array}$ & & $\begin{array}{l}\text { Protein kinase superfamily } \\
\text { protein }\end{array}$ \\
\hline 27424471 & $\begin{array}{l}\mathrm{C}>\mathrm{T} \\
\text { exon } 7\end{array}$ & 90.4 & Atlg72880 & $750 \mathrm{G}>\mathrm{A}$ & & $\begin{array}{l}\text { Survival protein SurE-like } \\
\text { phosphatase/ nucleotidase }\end{array}$ \\
\hline
\end{tabular}

${ }^{a}$ position refers to splicing variant one

b position refers to splicing variant two

c www.arabidopsis.org

Two gene models for $C R K 43$ were predicted in TAIR10. Sequencing of cDNA prepared from rosette leaves showed that CRK43 is spliced according to the CRK43.1 model (data not shown).

The guanine to adenine mutation identified in crk43-2 is considered as synonymous mutation as it is located in intron three (Figure 29). This mutation changes the " $A G$ " acceptor sequence at the end of intron three to an "AA". The highly conserved AG acceptor sequence at the end of an intron is necessary for correct intron-exon recognition (Lorković et al., 2000). Thus, mutation of this sequence might result in alternative splicing. To investigate if the CRK43 transcript is altered in crk43-2, RNA was isolated from single suppressor and non-suppressor plants (2.2.3.13). CRK43 cDNA was amplified and subsequently sequenced. Alignment of the sequencing results to the CRK43 WT cDNA sequence revealed that all investigated suppressor plants carried a $10 \mathrm{bp}$ deletion in exon four (Figure 29) whereas the investigated nonsuppressor plants did not show this deletion (data not shown). Obviously, the spliceosome simply recognized the next AG in the pre-mRNA as acceptor sequence (Figure 29). The 10 bp deletion results in a frameshift and a premature stop codon in the protein (Figure 29). The stop codon is located between kinase subdomains V and VIa, leading to a truncated protein lacking more than half of the kinase domain. This strongly suggests that the crk43-2 gene product is unlikely to have kinase activity. 

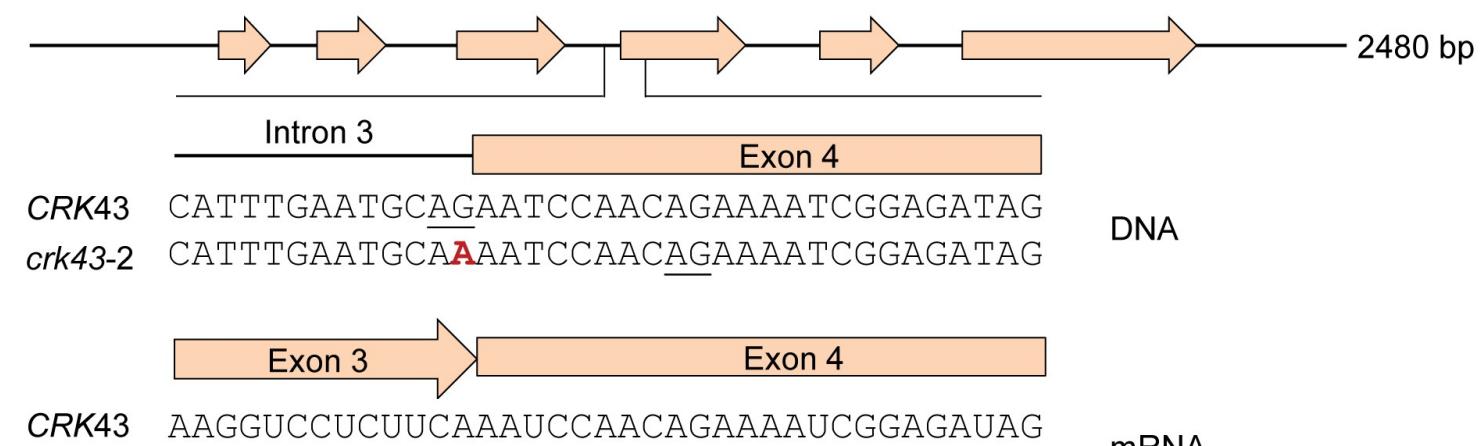

CRK43 AAGGUCCUCUUCAAAUCCAACAGAAAAUCGGAGAUAG

crk43-2 AAGGUCCUCUUCA----------AAAAUCGGAGAUAG

mRNA

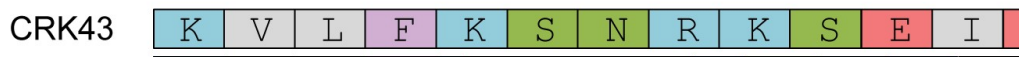

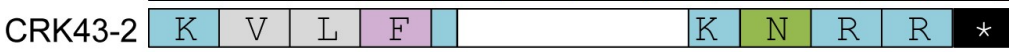

Protein

V. Vla. Kinase domain $425 \mathrm{AA}$

Figure 29: The crk43-2 mutation identified in the cerk1-4 suppressor screen.

Schematic structure of the CRK43 gene, transcript and protein is shown. Exons are depicted as light orange arrows. The DNA sequence alignment shows the guanine $e^{446-1}$ to adenine nucleotide exchange (red) in $c r k 43-2$. The spliceosome acceptor sequences (AG) at the end of intron three are underlined in the WT and crk43-2 sequences. The crk43-2 transcript is aligned to the CRK43 wild type sequence and the missing bases are highlighted in red. Predicted protein features: Kinase domain and low complexity region (grey box). The light grey boxes in the kinase domain highlight kinase subdomains V and VIa. Domains were predicted using SMART (http://smart.embl-heidelberg.de/, Letunic and Bork, 2018). Color code for amino acids: Polar positive $=$ blue, polar negative $=$ red, polar neutral $=$ green, non-polar aliphatic $=$ grey, non-polar aromatic $=$ purple. $*=$ stop codon.

The hypersensitive cell death reaction of cerk1-4 plants is SA dependent (Petutschnig et al., 2014). Therefore, similar to the experiments conducted for $C R K 7$ and $c r k 7-4$ (3.1.1), expression of the SA marker gene PRl was investigated in crk43 mutant plants. To obtain a crk43-2 single mutant, cerkl-4 crk43-2 was crossed with Col-3 gll. The crk43-2 single mutant was included in the experiment to examine if this mutation causes a cerk1-4 independent phenotype. PRI expression was investigated in uninfected and Bgh infected crk43-2 and cerk1-4 crk43-2 plants. Col-3 gll and cerk1-4 were included in the experiment as controls. RNA was extracted (2.2.3.13) from a pool of ten plants per line and PRI as well as ACTIN were amplified (2.2.3.15).

As described before (Petutschnig et al., 2014), PRl expression in Bgh infected cerkl-4 plants was higher than in infected WT plants. PRI expression levels in cerk1-4 crk43-2 and crk43-2 plants were on the same level as in Col-3 gll (Figure 30). This suggests that CRK43 is necessary for the accumulation of SA to a higher level in cerkl-4 plants. 
A

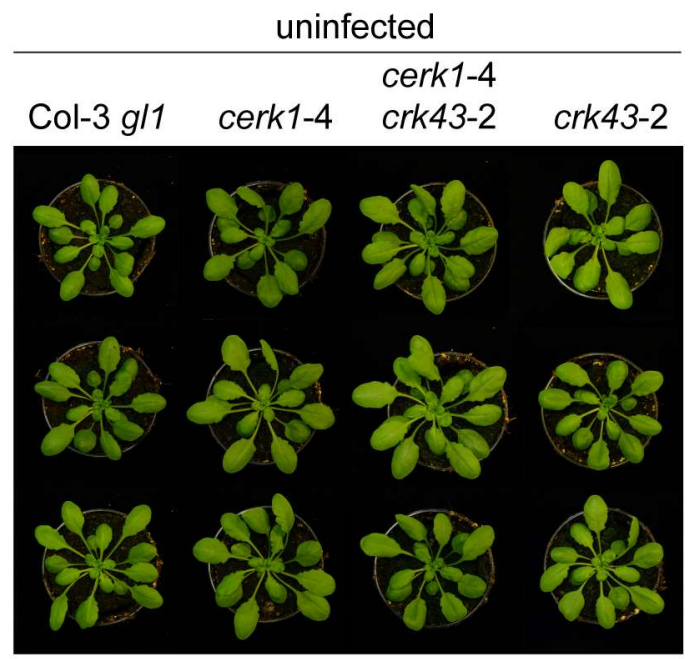

Bgh 7 dpi

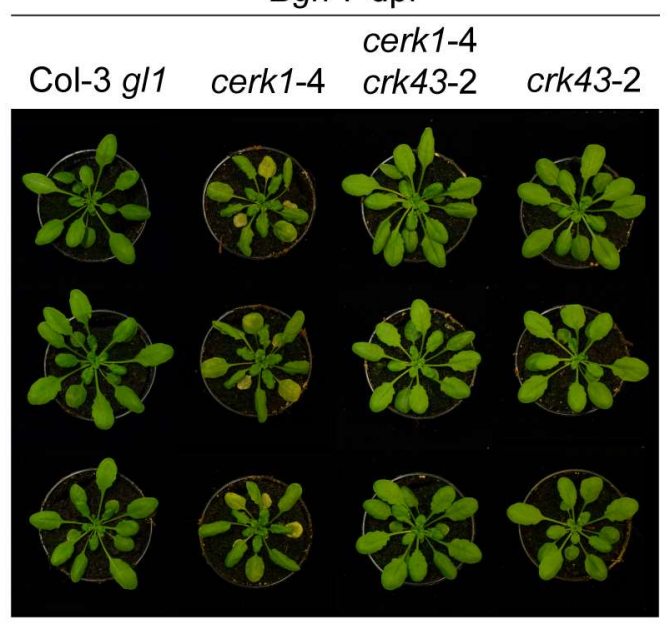

B

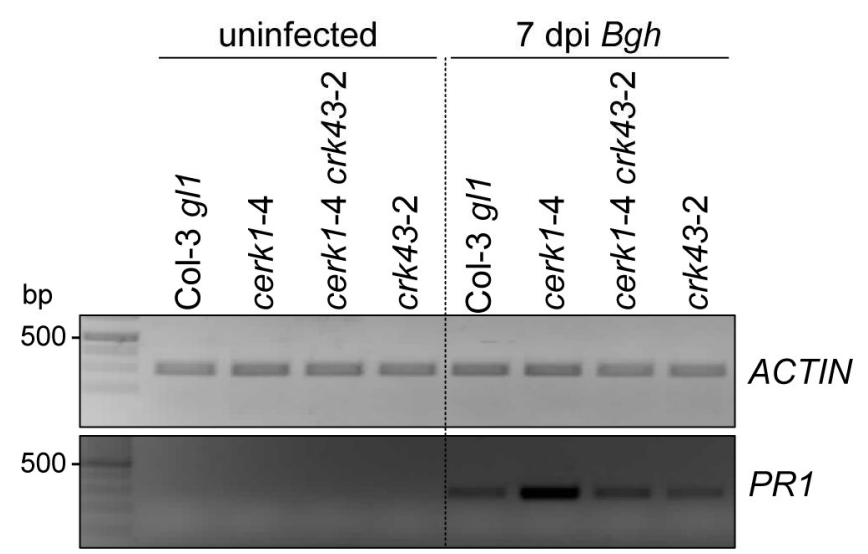

Figure 30: A mutation in CRK43 suppresses increased powdery mildew induced PR1 gene expression in cerk1-4.

4.5 week-old plants were either infected with Blumeria graminis f.sp. hordeii (Bgh) or kept uninfected. Whole rosettes of ten infected plants per line were harvested 7 dpi. Semi-quantitative RT-PCR was performed on PRI with ACTIN as a control. A: Representative composite images of uninfected and infected plants. Pictures were taken by L. Pierdzig. B: Semi-quantitative RT-PCR. This experiment was conducted in three independent biological replicates with similar results.

\subsubsection{Confirmation of CRK43 as a molecular component of cerkl-4 cell death induction}

To confirm crk43-2 as the suppressor mutation in noce8-1, crk43 T-DNA mutant lines were ordered, genotyped and analyzed regarding their ability to suppress the cerkl-4 cell death response.

For complementation experiments it was tested if expression of CRK43 and/ or CRK43mCitrine in cerk1-4 crk43-2 plants re-establishes cell death. 


\subsubsection{Analysis of crk43 T-DNA insertion lines}

\subsection{Isolation of the crk43-1 and crk43-3 T-DNA insertion lines}

Five different CRK43 T-DNA insertion lines were ordered. Genotyping and analysis of TDNA flanking sequences in these lines revealed that T-DNA insertions in SALK030875, SALK024283 and SALK091320 are at the same position upstream of the 5'UTR. Two of these lines were included in a previous study and named crk43-1 (SALK024283) and crk43-2 (SALK030875) respectively (Bourdais et al., 2015). As our sequencing results showed that these lines are identical, we named all three lines crk43-1 (Figure 31A and B).

Two more T-DNA insertion lines, SALK201662 and SALK078417, were genotyped. The TDNA insertion in both lines is localized at the same position at the beginning of the first intron (Figure 31A and C). As both lines are identical, they were named crk43-3. The T-DNA insertion in crk43-3 disrupts the gene before the region encoding the kinase domain (Figure 31D).

To investigate if CRK43 expression is altered in crk43-1 (SALK201662) and/ or crk43-3 (SALK091320), RNA was extracted (2.2.3.13) from uninfected and E. cruciferarum infected crk43-1, crk43-3 and Col-0 control plants. RT-PCRs using the primers indicated in figure $31 \mathrm{~A}$ and primers amplifying $A C T I N$ as internal control were conducted (Figure 31E; 2.2.3.15). Expression of $C R K 43$ was not altered in crk43-1, confirming the findings of a previous study (Bourdais et al., 2015).

In crk43-3 no CRK43 transcript was detectable using primers spanning the T-DNA insertion (Figure 31E). Therefore, crk43-3 can be considered as a crk43 knockout mutant. 


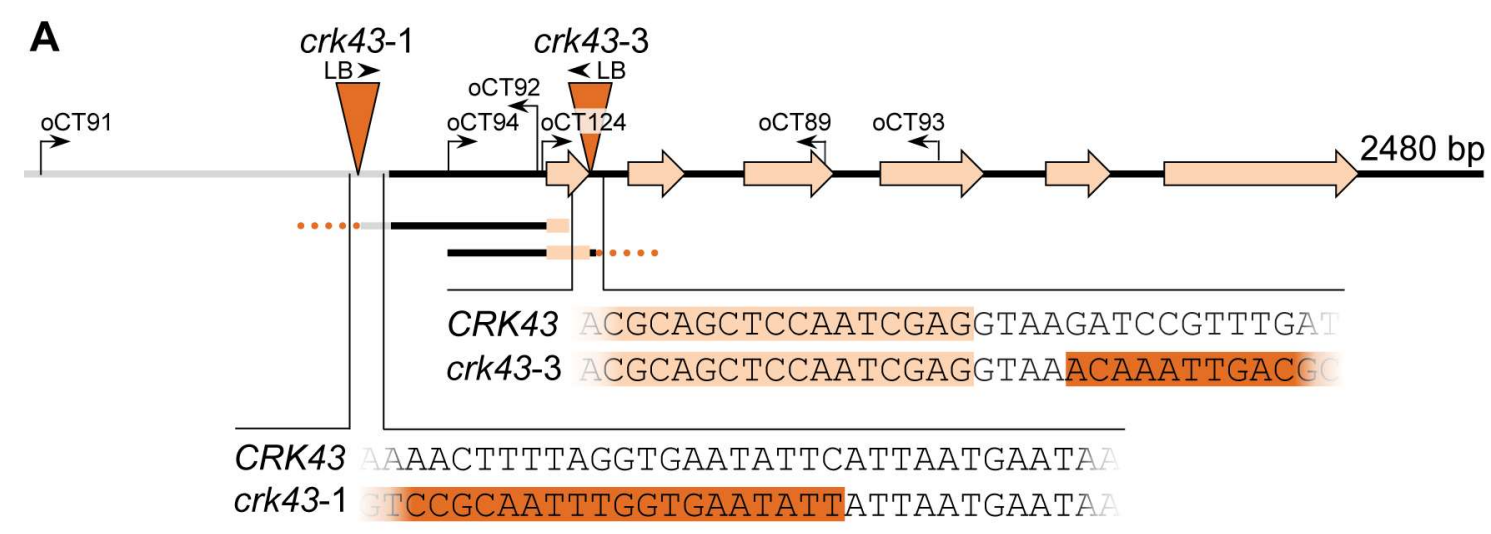

B

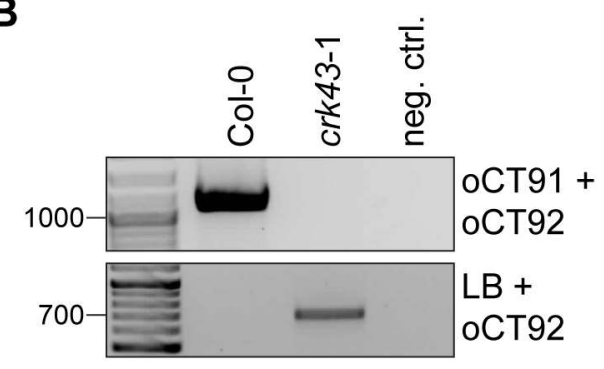

D

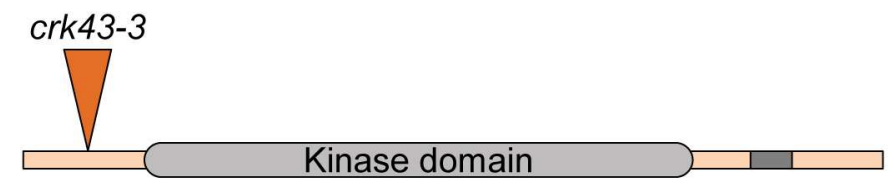

425 AA
C

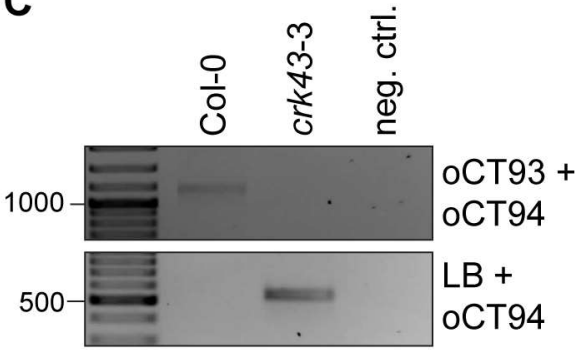

crk43-3

E

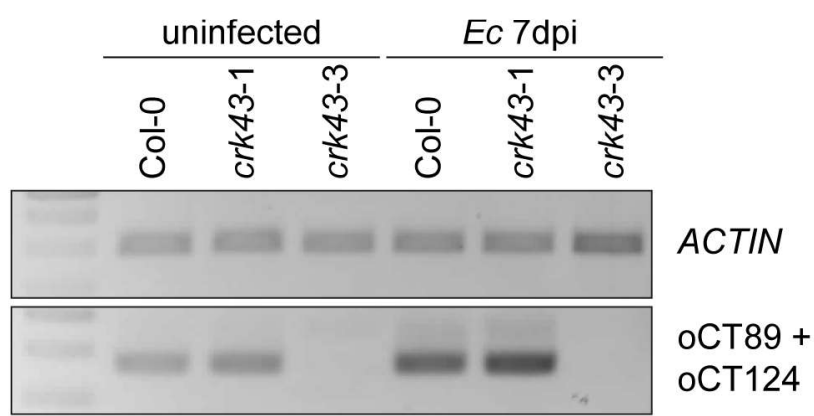

Figure 31: Characterization of the crk43-1 and crk43-3 T-DNA insertion lines used in this study. A: Schematic structure of the $C R K 7$ gene. Exons are depicted as light orange arrows. Primers used for genotyping are indicated with black arrows. The T-DNA insertion is depicted as a dark orange triangle; $\mathrm{LB}=$ Left border. PCR products shown in $\mathrm{B}$ and $\mathrm{C}$ were sequenced; a section of the sequencing results is shown. Light orange areas align to exons, dark orange areas align to the T-DNA. B + C: PCR-based genotyping of crk43-1 (B) and crk43-3 (C). Homozygosity was verified using the primers indicated in A. PCR products were sequenced and sequencing results are shown in A. D: Schematic structure of the CRK43 protein. Predicted protein features: Kinase domain and low-complexity region (grey box). Domains were predicted using SMART (http://smart.embl-heidelberg.de/, Letunic and Bork, 2018). The position of the crk43-3 T-DNA insertion is shown with an orange triangle. E: Semi-quantitative reversetranscription PCR with the indicated primers was performed on RNA extracted from pools of nine uninfected or nine E. cruciferarum infected plants per line. dpi = days post infection. 
3.2.2.1.2 The crk43-3 T-DNA insertion suppresses the cerk1-4 cell death phenotype

In order to obtain a cerk1-4 crk43-3 double mutant, cerk1-4 was crossed with crk43-3 (2.2.1.1.5). A population of plants derived from this cross homozygous for cerk1-4 but segregating for crk43-3 was infected with E. cruciferarum together with a line homozygous for cerk1-4 and segregating for crk43-2. The control plants Col-3 gll, cerk1-4, crk43-2, Col-0 and crk43-3 were included in the experiment. All segregating plants were genotyped for crk43-2 and crk43-3 respectively. Infected plants were scored regarding cell death formation as well as fungal growth and pictures of the infected plants were taken nine dpi. Representative images are shown in figure 32.
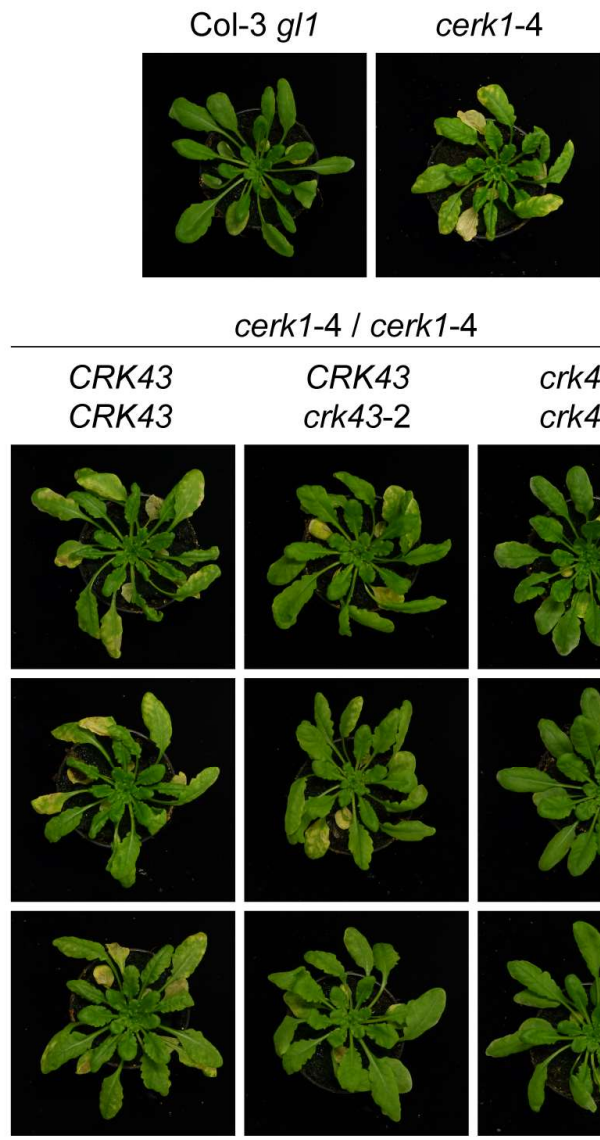

cerk1-4 / cerk1-4

CRK43

$$
\text { crk43-2 }
$$
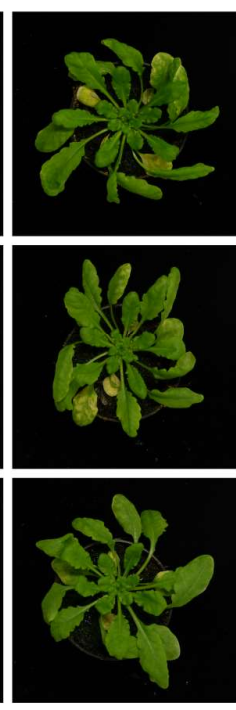

crk43-2

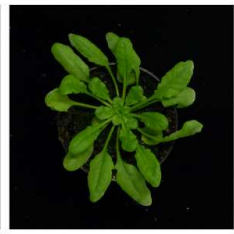

Col-0

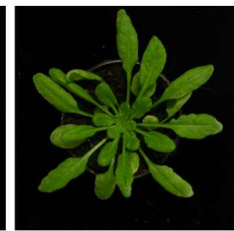

crk43-3

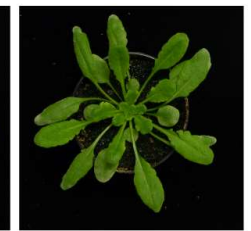

cerk1-4 / cerk1-4

\begin{tabular}{lll}
\hline CRK43 & CRK43 & crk43-3 \\
CRK43 & crk43-3 & crk43-3
\end{tabular}
crk43-2
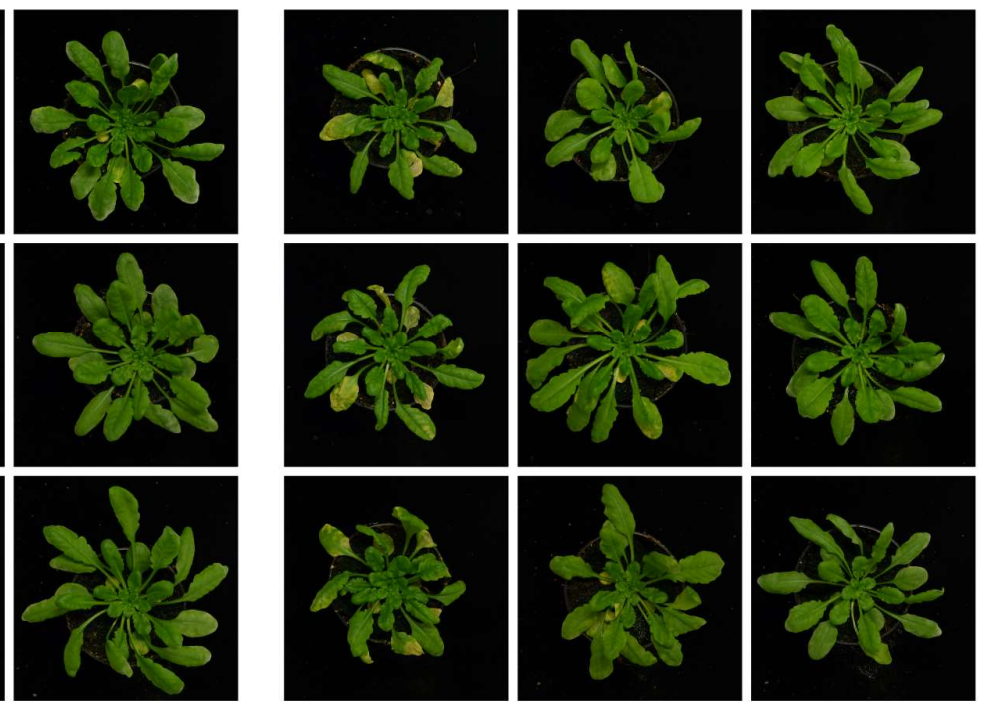

Figure 32: cerk1-4 crk43-2 and cerk1-4 crk43-3 show semi-dominant cerk1-4 suppressor phenotypes upon $E$. cruciferarum infection.

Four-week-old plants were randomized and infected with E. cruciferarum. Pictures of infected plants were taken nine days post infection (dpi) by L. Pierdzig and images of three representative plants are shown per genotype. Genotypes are indicated above the pictures.

As expected, plants homozygous for cerkl-4 and WT for CRK43 showed the cerkl-4 cell death phenotype. Plants homozygous for either the $c r k 43-2$ mutation from the screen or the 
crk43-3 T-DNA insertion showed a full suppressor phenotype whereas cerkl-4 plants heterozygous for crk43-2 or crk43-3 displayed the intermediate phenotype with a low amount of fungal growth and only mild cell death (Figure 32). This confirms crk43-2 as the suppressor mutation in noce8-1 and crk43-3 as a CRK43 knockout line.

crk43-1 was also crossed with cerk1-4, but F2 plants carrying the cerk1-4 mutation and homozygous for the crk43-1 T-DNA insertion showed cerk1-4 cell death after E. cruciferarum infection (data not shown). This matches the results that CRK43 expression is not altered in this line (3.2.2.1.1).

\subsubsection{Expression of CRK43 in cerk1-4 crk43-2 induces cell death}

crk43-2 was confirmed as the suppressor mutation in noce8-1 by analysis of a crk43 T-DNA insertion line (3.2.2.1). Additionally, classical complementation experiments were conducted. For complementation, $p C R K 43:: C R K 43$ was transformed into cerk1-4 crk43-2 plants via Agrobacterium mediated floral dip. At the age of four weeks, 18 of 56 (32\%) T1 plants developed very strong cell death leading to growth arrest and/ or plant death at the seedling stage (stage 1.02-1.04 according to Boyes et al., 2001) (Figure 33). $41 \%$ of the T1 plants showed dwarf phenotypes and the remaining $27 \%$ were WT-like (Figure 33). These results suggest that $C R K 43$ expression in cerk1-4 crk43-2 triggers a strong cell death response that can exceed the cell death seen in cerk1-4.

Similar to the results observed for untagged CRK43, also expression of CRK43-mCitrine in cerk1-4 crk43-2 under the native promotor triggered a strong cell death reaction in 21 of 46 (46\%) T1 plants (Figure 33). This suggests that the CRK43-mCitrine protein is functional. $30 \%$ of the T1 plants showed a dwarf phenotype and the remaining $24 \%$ were WT-like (Figure 33). CRK43 expression seems to have a dosis dependent effect as most probably plants expressing CRK43 at a high level are those that died. As these plants were dead, no experimental proof of this hypothesis was possible. In both constructs CRK43 was expressed under the native promotor, nevertheless different expression levels are possible depending on the insertion site of the T-DNA. Expression of the construct in plants still alive was confirmed via confocal laser scanning microscopy (3.2.4.1). 


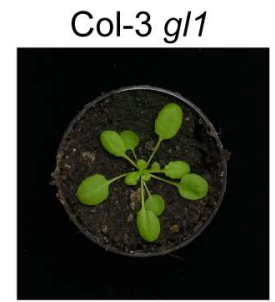

small dead

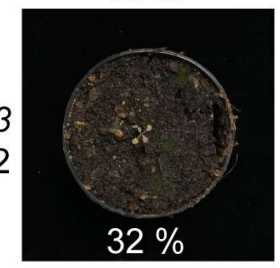

pCRK43::CRK43 in cerk1-4 crk43-2

pCRK43::CRK43-mCit in cerk1-4 crk43-2

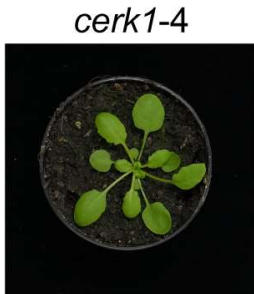

dwarf no/ slight CD

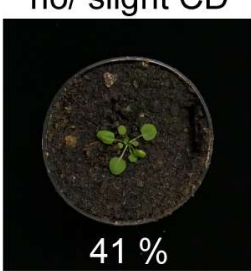
cerk1-4 crk43-2

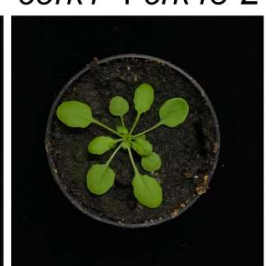

WT-like

no CD
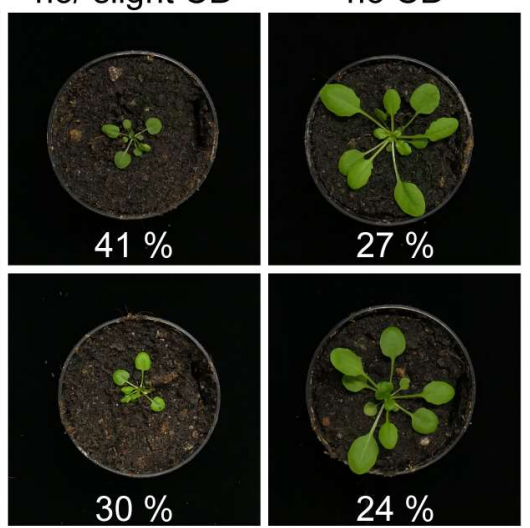

Figure 33: Expression of CRK43 and CRK43-mCitrine triggers cell death in uninfected cerk1-4 crk43-2 plants.

pCRK43::CRK43 and $p C R K 43:: C R K 43-m$ Citrine ( $m$ Cit) were transformed into cerk1-4 crk43-2 via Agrobacterium mediated floral dip. T1 plants were selected via Basta ${ }^{\circledR}$ treatment. Pictures of uninfected four-week old control (upper panel) and T1 plants (lower panels) were taken. Representative images for every genotype and phenotype are shown. Categories for scoring are indicated above the T1 plant pictures. Percentages of plants with these phenotypes on the basis of 56 (pCRK43::CRK43) or 46 ( $p C R K 43:: C R K 43-m C i t)$ analyzed T1 plants are shown below the plant pictures.

Plants that developed past the seedling stage were randomized with the control lines Col-3 gll, cerk1-4 and cerk1-4 crk43-2 prior to E. cruciferarum infection. The majority of cerk1-4 crk43-2 plants transformed with CRK43 or CRK43-mCitrine developed strong cerk1-4 cell death phenotypes upon infection. The observed cell death reaction was stronger than in the cerk1-4 control plants six days post infection. Cell death was observed in plants with and without dwarf phenotypes. In figure 34 representative images of plants that did not show cell death at the age of four weeks, but developed strong cell death six days post E. cruciferarum infection, are shown. Taken together, these results confirm that CRK43 is involved in cerk1-4 cell death induction and triggers a strong cell death in uninfected plants possibly due to high expression levels. 
A
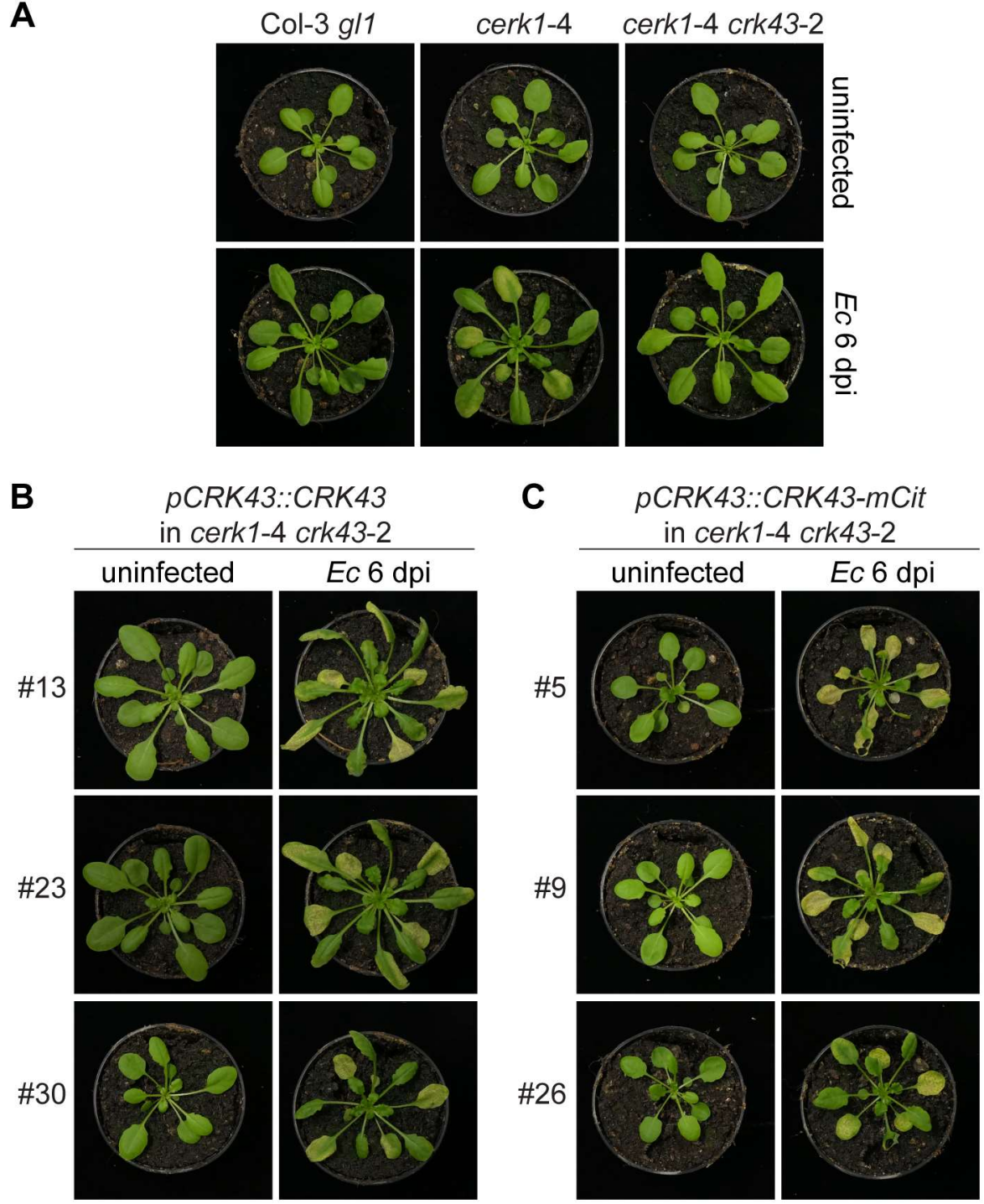

Figure 34: CRK43 and CRK43-mCitrine expression in cerk1-4 crk43-2 re-establishes the cerk1-4 cell death.

$p C R K 43: \because C R K 43$ and $p C R K 43:: C R K 43-m$ Citrine ( $m$ Cit) were transformed into cerkl-4 crk43-2 via Agrobacterium mediated floral dip. T1 plants were selected via Basta ${ }^{\circledR}$ treatment. Four-week-old T1 plants and the according controls were infected with E. cruciferarum. Pictures were taken before infection and 6 dpi. Line numbers (\#) are indicated next to the images. A: Control plants. B: $p C R K 43: \because C R K 43$ in cerk1-4 crk43-2. C: $p C R K 43:: C R K 43-m$ Citrine in cerk1-4 crk43-2.

\section{$\underline{3.2 .3 \text { crk43 mutant lines show normal early PAMP responses }}$}

In order to test if CRK43 is involved in PAMP triggered responses, it was investigated if CERK1 phosphorylation, ROS burst induction and MAPK activation upon chitin treatment are impaired in crk43 mutants.

For other members of the CRK family, interaction with the FLS2-BAK1 protein complex and a contribution to flg22 signaling was shown (Yadeta et al., 2017; Yeh et al., 2015). Therefore, early PAMP responses after flg22 treatment were investigated additionally. 
The experiments were performed with $c r k 43-2$, the mutant identified in the suppressor screen (3.2.1) and the crk43-1 and crk43-3 T-DNA insertion lines (3.2.2). Col-3 gll, cerkl-4 and Col-0 were included in the experiments as controls as they are the background of crk43-2 (Col-3 gll), cerk1-4 crk43-2 (cerk1-4) and crk43-1/ crk43-3 (Col-0) respectively. cerk1-2 and $f l s 2$-c knockout lines were used as negative controls.

\subsubsection{CERK1 phosphorylation upon chitin perception is not impaired in crk43 mutants}

The CERK1 receptor is phosphorylated upon chitin binding and this phosphorylation is visible through a band-shift in Western Blot analysis with a CERK1-specific antibody (Petutschnig et al., 2010). A CERK1 band shift assay upon chitin treatment was performed with crk43 mutant plants to investigate if CRK43 is necessary for CERK1 phosphorylation. For this, Arabidopsis leaves of crk43-2, crk43-1 and crk43-3 and their respective controls as well as cerk1-2 as negative control were vacuum-infiltrated with either water or $100 \mu \mathrm{g} / \mathrm{ml}$ chitin and incubated for $10 \mathrm{~min}$ after the infiltration (2.2.1.1.8). The adjusted protein extracts were used for Western Blot analysis (2.2.4.5) with a CERK1-specific antibody. All investigated lines showed a band-shift after chitin treatment at a comparable level (Figure 35), suggesting that the CERK1 phosphorylation is independent from CRK43.

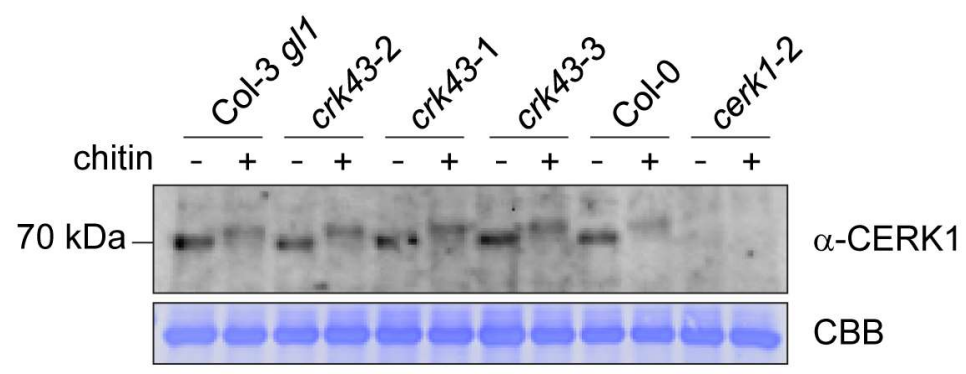

Figure 35: crk43 mutants show a WT-like CERK1 band shift upon chitin treatment.

Arabidopsis leaves of the indicated lines were vacuum-infiltrated for ten minutes with water or $100 \mu \mathrm{g} /$ $\mathrm{ml}$ chitin. Infiltrated samples were incubated ten minutes at RT before being frozen. Western Blot with the adjusted total protein extracts was performed using a CERK1-specific antibody. Phosphorylation is visible in all lines except for the CERK1 knockout mutant cerk1-2 with a band shift. The experiment was conducted twice with similar results by P. Lude during Bachelor thesis supervised by C. Trippel.

\subsubsection{2 crk43 mutants show WT-like PAMP induced ROS accumulation}

Accumulation of extracellular ROS is one of the early responses upon PAMP perception. In Arabidopsis the NADPH oxidases RBOHD and RBOHF, which are specifically activated upon PAMP perception, generate ROS into the apoplast (Torres et al., 2005). To investigate 
if the ability to induce ROS accumulation upon PAMP treatment is altered in crk43 mutant lines, leaf discs of Arabidopsis plants were treated with $100 \mathrm{nM}$ flg22, $10 \mu \mathrm{g} / \mathrm{ml}$ chitin or PAMP-free luminol-solution as negative control (2.2.3.17).

None of the investigated crk43 mutant lines showed an altered ROS burst upon either chitin (Figure 36A) or flg22 (Figure 36B) treatment. Therefore, CRK43 seems not to play a role in ROS burst induction upon PAMP perception. As expected, cerk $1-2$ and $f l s 2$-c did not show a ROS burst upon chitin and flg22 treatment, respectively.
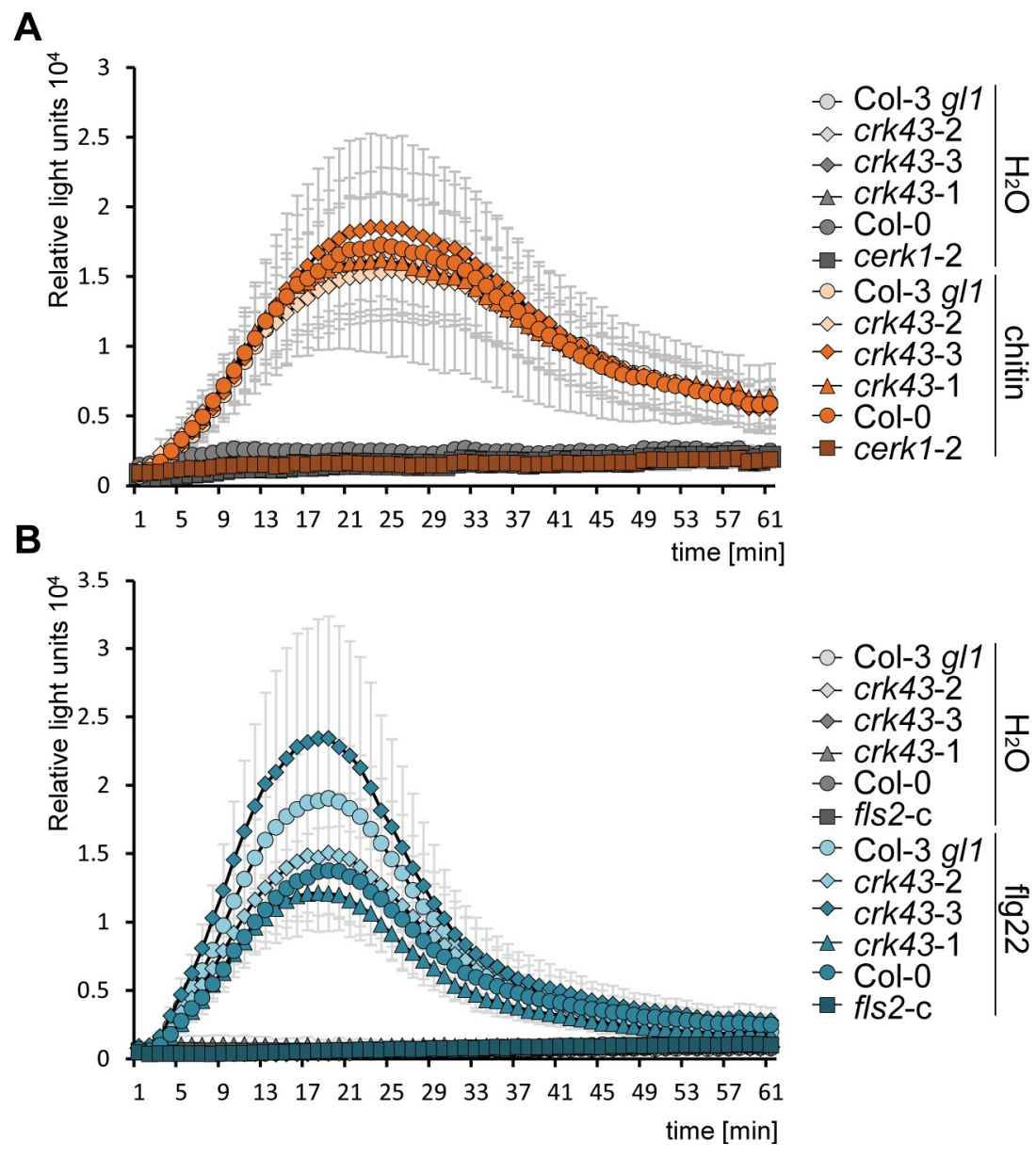

\begin{abstract}
Figure 36: ROS burst upon chitin and flg22 treatment is not altered in crk43 mutant lines.
Eight leaf discs per line of five- to seven-week-old Arabidopsis plants were treated with either luminol solution without PAMP $\left(\mathrm{H}_{2} \mathrm{O}\right)$, polymeric chitin $(100 \mu \mathrm{g} / \mathrm{ml}$, A) or flagellin22 (flg22, B). Relative light units were measured every minute for $60 \mathrm{~min}$ after the respective treatment. Data show mean of eight leaf discs and error bars represent StDev. The experiments were performed in four (A) or three (B) replicates with similar results by P. Lude during Bachelor thesis supervised by C. Trippel.
\end{abstract}

\title{
3.2.3.3 crk43 mutants show WT-like PAMP induced MAPK activation
}

For signal transduction upon PAMP perception, MAPK cascades are activated that regulate downstream components of the plant immune system. To address the question if MAPK 
phosphorylation is altered in crk43 mutants compared to the according WT controls, 14-dayold in vitro grown seedlings were treated for twelve minutes with $10 \mu \mathrm{g} / \mathrm{ml}$ chitin, $50 \mathrm{nM}$ flg22 or medium without PAMPs (2.2.1.1.2). Protein extracts were analyzed via Western Blot (2.2.4.5) using the $\alpha-p 42 / 44-M A P K$ antibody that specifically detects phosphorylated and therefore active MAPKs. As expected, no MAPK phosphorylation was induced by treatment with medium only (Figure 37A). After both flagellin (Figure 37B) and chitin (Figure 37C) treatment, MAPK activation was observed in all investigated crk43 mutant lines similar to their according WT controls. These results indicate that CRK43 does not play a role in MAPK phosphorylation upon PAMP perception. As expected, cerkl-2 and fls2-c did not show a MAPK phosphorylation upon chitin and flg22 treatment respectively.

A

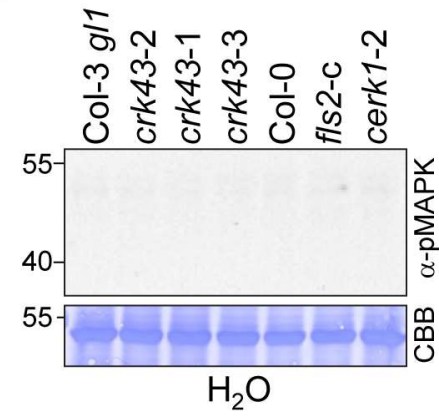

B

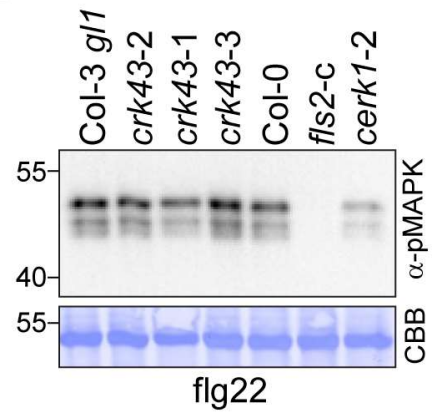

C

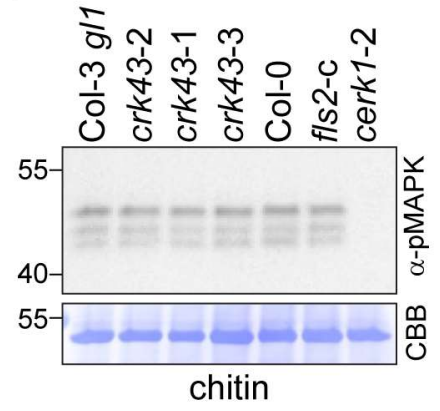

Figure 37: crk43 mutants show WT-like MAPK activation upon flg22 and chitin treatment.

14-day-old seedlings of crk43-2, crk43-1 and crk43-3 mutants and the according controls were treated for 12 minutes with either PAMP-free medium $\left(\mathrm{H}_{2} \mathrm{O}, \mathbf{A}\right), 100 \mathrm{nM}$ flagellin22 (flg22) (B) or $10 \mathrm{mg} \mathrm{ml}^{-1}$ polymeric chitin $(\mathbf{C})$. Immunoblotting was performed with the p42/44- $\alpha$-MAPK antibody ( $\alpha$-pMAPK) detecting phosphorylated MAPKs. fls2-c served as negative control for flg22 perception, cerk1-2 as negative control for chitin perception. CBB: Coomassie Brilliant Blue-stained membranes. This experiment was performed in four replicates with similar results by P. Lude during Bachelor thesis supervised by C. Trippel.

\subsubsection{Analysis of CRK43 subcellular localization}

CRK43 is a truncated CRK lacking the entire ectodomain and should be therefore considered as a receptor-like cytoplasmic kinase (Bourdais et al., 2015). No myristyolation sites or other lipid modifications are predicted suggesting that CRK43 localizes to the cytosol (ARAMEMNON, Schwacke et al., 2003). CRK43-mCitrine localization was investigated after transient expression in N. benthamiana and after stable expression in Arabidopsis T1 plants. 
3.2.4.1 Analysis of CRK43-mCitrine localization after transient expression in N. benthamiana

In order to investigate the subcellular localization of CRK43, co-infiltration experiments of pCRK43::CRK43-mCitrine with cellular markers were conducted in $N$. benthamiana. pUBQ10::mKate2-SYP122 was used as a plasma membrane marker (E. Petutschnig, unpublished), pUBQ10::mKate2-N7 (H. Ghareeb, unpublished) as nuclear marker and p35S::ER-ck (Nelson et al., 2007) as an ER marker. Confocal laser scanning microscopy (Leica SP8) was performed two to three days post infiltration (Figure 38). In general, the CRK43-mCitrine fluorescence signal was very weak, suggesting low CRK43-mCitrine expression levels in $N$. benthamiana. A weak CRK43-mCitrine fluorescence was detectable in the cell periphery and in cytoplasmic strands. The cell periphery signal co-localized with the plasma membrane marker mKate2-SYP122 (Figure 38A).

A stronger CRK43-mCitrine fluorescence was observed in the nucleus and particularly in the nucleolus. The nuclear signal was confirmed by co-localization with the nuclear marker mKate2-N7. Also, nucleolar localization of CRK43-mCitrine could be confirmed in this assay, since mKate2-N7 marks the nucleus but not the nucleolus (Figure 38B).

Since the ER is present in cytoplasmic strands, these are visible in cells infiltrated with ERck. This fluorescence signal overlaps with the CRK43-mCitrine signal, confirming CRK43mCitrine localization to the cytoplasm (Figure 38C).

These experiments reveal that CRK43-mCitrine is localized to the cytoplasm, cell periphery, nucleus and nucleolus in $N$. benthamiana. 


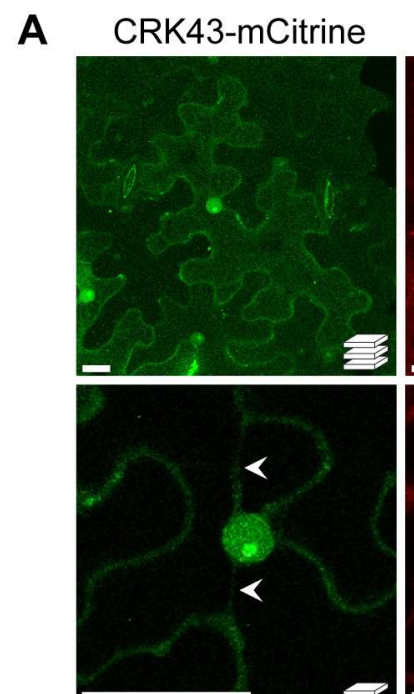

mKate2-SYP122

overlay

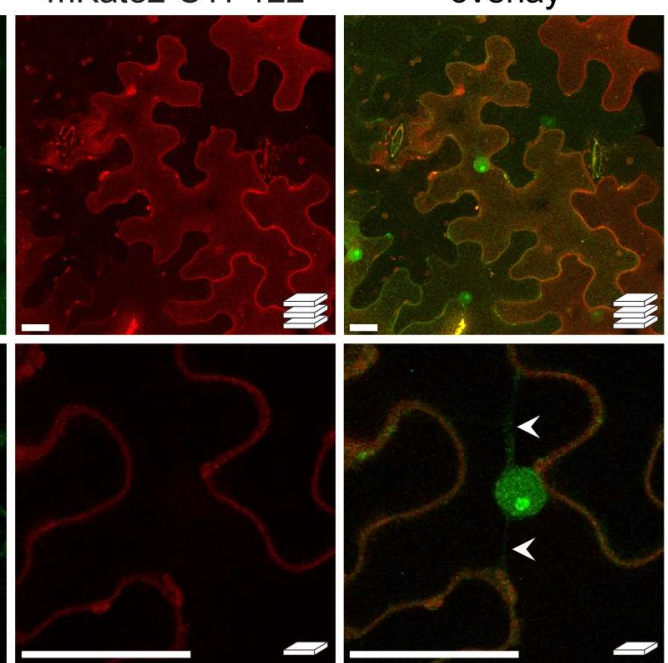

B

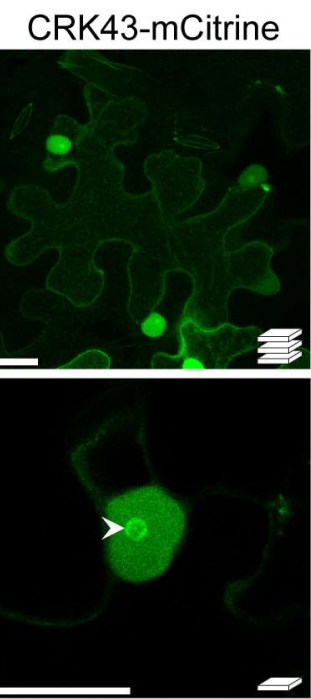

mKate2-N7

overlay
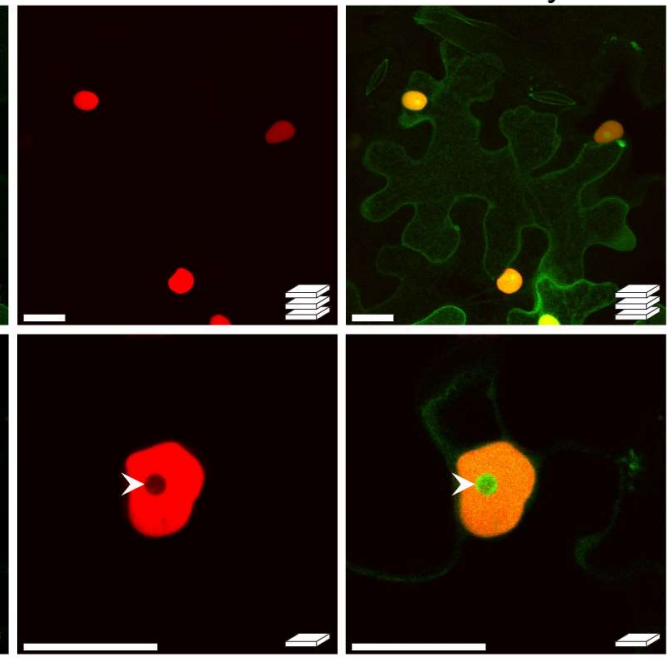

C

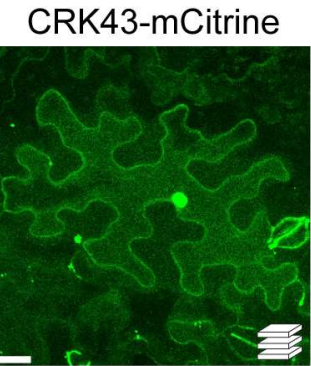

ER-ck

overlay
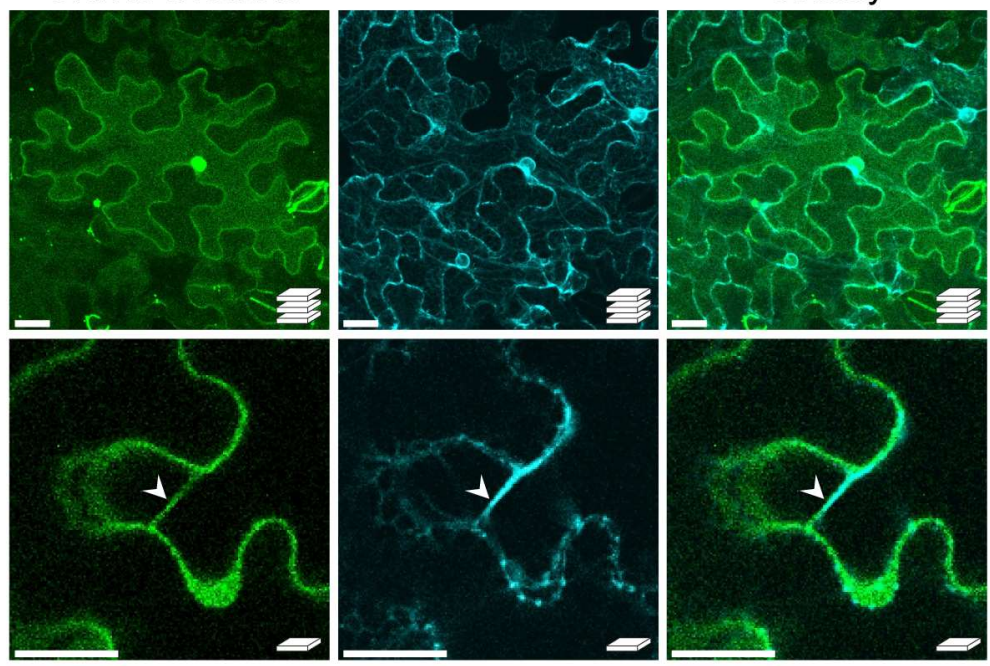

Figure 38: CRK43-mCitrine co-infiltrated with cellular markers in $N$. benthamiana.

A. tumefaciens cultures transformed with $p C R K 43: \because C R K 43-m C i t r i n e$ were mixed with cultures transformed with $p U B Q 10:: m$ Kate2-SYP122, pUBQ10::mKate2-N7 or $p 35 S:: E R$-ck prior to infiltration into $N$. benthamiana. Confocal microscopy (Leica SP8) was performed 2-3 dpi. A to C show co-expression of CRK43-mCitrine (green) with mKate2-SYP122 (red; E. Petutschnig, unpublished; A), mKate2-N7 (red; 
H. Ghareeb, unpublished; B) or ER-ck (cyan; Nelson et al., 2007; C). Images are representative maximum projections or single plain images as indicated. Scale bar $=25 \mu \mathrm{M}$; Z-stack sizes $18-25 \mu \mathrm{M}$, line accumulation 3-6. Arrow heads point to cytoplasmic strands (A and C) or to the nucleolus (B).

\subsubsection{Analysis of CRK43-mCitrine localization in Arabidopsis}

Expression of CRK43-mCitrine in cerkl-4 crk43-2 led to strong cell death in some uninfected plants and induced cell death stronger than in cerk1-4 control plants upon infection with $E$. cruciferarum (3.2.2.2). This confirms functionality of the CRK43-mCitrine construct. CRK43-mCitrine expression was investigated in 24 uninfected T1 plants and in the same plants six days post infection with E. cruciferarum. As observed in N. benthamiana (3.2.4.1), the CRK43-mCitrine fluorescence signal was generally very low, making confocal imaging very difficult. E. cruciferarum infection increased the CRK43-mCitrine fluorescence signal to some extent, suggesting increased CRK43 expression upon E. cruciferarum infection. This finding should be investigated in detail in future experiments. However, it is in line with the observation that $C R K 43$ is upregulated upon Bgh infection (Bourdais et al., 2015), but contrary to publically available expression data that show downregulation of $C R K 43$ expression in response to Golovinomyces orontii infection (Chandran et al., 2010).

Since the signals were generally weak, correlation between cell death and CRK43-mCitrine signal intensity could not be analyzed.

CRK43-mCitrine localized predominantly to the cell periphery in Arabidopsis (Figure 39). Additionally, in some cells nuclear localization was observed (Figure 39). As this experiment was conducted only once due to time limitations of this work, localization of CRK43mCitrine in Arabidopsis needs to be investigated in more detail in the T2 generation. 


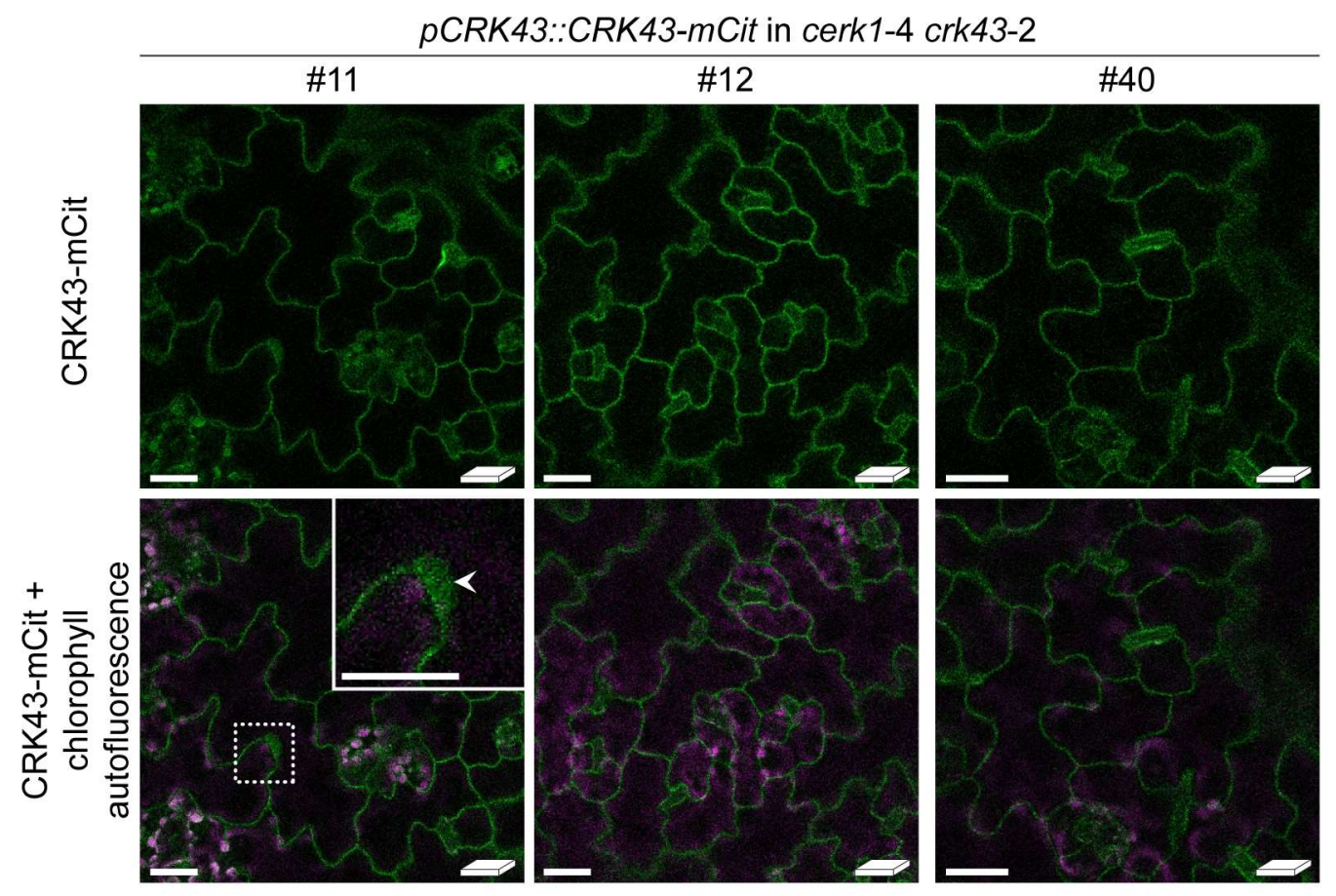

Figure 39: CRK43-mCitrine localization in cerk1-4 crk43-2 T1 plants.

Confocal microscopy (Leica SP5) was performed with E. cruciferarum infected stably transformed Arabidopsis T1 plants expressing $p C R K 43: \because C R K 43-m$ Citrine in cerk1-4 crk43-2 6 dpi. Representative images of three independent T1 lines are shown. Green= CRK43-mCitrine (mCit); Purple= chlorophyll autofluorescence. Scale bar $=25 \mu \mathrm{M}$. Line accumulation=2. Inset picture shows details, arrowhead points to the nucleus.

\subsection{Isolation of a novel pad4 mutation that is able to suppress cerk1-4 dependent cell}

\section{death}

In the forward genetic screen to identify molecular components required for cerkl-4 cell death formation another batch was investigated. The M2 line noce12-23 was identified as suppressor (L. Pierdzig and C. Trippel), propagated to the M3 generation and crossed with cerk1-4. 193 F2 plants of this cross were infected with E. cruciferarum. $28 \%$ showed a clear suppressor phenotype with a stronger fungal growth on the leaf surface than on Col-3 gll control plants. The remaining $72 \%$ developed cell death as in cerk1-4 control plants (Figure 40). These results suggest that the suppressor mutation in noce12-23 is recessive. 


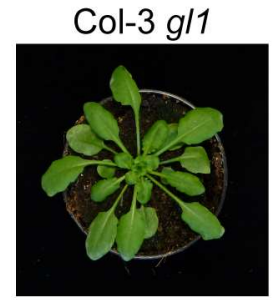

cerk1-4

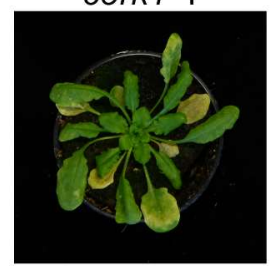

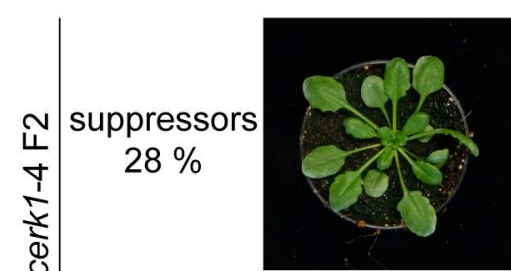
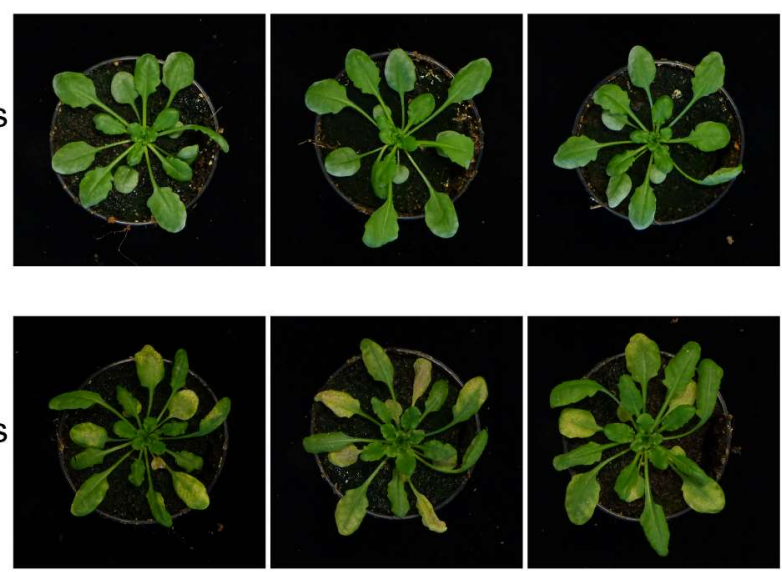

Figure 40: $E$. cruciferarum infected noce12-23 x cerk1-4 F2 plants show a recessive phenotype.

Four-week old noce12-23 x cerk1-4 F2 plants and the according controls Col-3 gll and cerk1-4 were infected with E. cruciferarum. Pictures were taken 7 dpi. A total number of 193 plants was scored according to cell death and fungal growth. Fungal growth on suppressor plants was higher than on Col-3 gll control plants and non-suppressors developed cell death similar to the cerk1-4 control. Numbers below the categories show the percentage of plants with the according phenotypes.

Leaves of all suppressors were pooled and DNA was extracted from this pool. DNA was used for $150 \mathrm{bp}$ paired-end Illumina sequencing and reads were aligned to the TAIR10 reference genome using CLC genomics workbench 10.1.1 (2.2.5.1). SNPs in noce12-23 were identified and SNPs that occurred in both the background line cerkl-4 and noce12-23 were removed. Available sequencing data from three independent suppressor lines (M. Stolze, C. Meusel, E. Petutschnig, C. Trippel) were compared to the SNPs identified in noce12-23 and duplicates were removed to obtain noce12-23 specific SNPs. These SNPs were plotted against their chromosomal position (Figure 41). As explained before (3.1.1 and 3.1.2) SNP frequencies at the centromere are elevated but the majority of these SNPs can be considered as artefacts due to alignment problems of repetitive sequences. Since only suppressor plants were included in the pool for mapping, all plants should carry the suppressor mutation resulting in a SNP frequency of $100 \%$. Unlinked SNPs have an expected SNP frequency of about $50 \%$ whereas genetically linked SNPs should show an elevated SNP frequency increasing with proximity to the causative mutation. Hence, the genome region carrying the suppressor mutation should display a SNP frequency peak. In noce12-23, such a peak was apparent on chromosome three (Figure 41). 

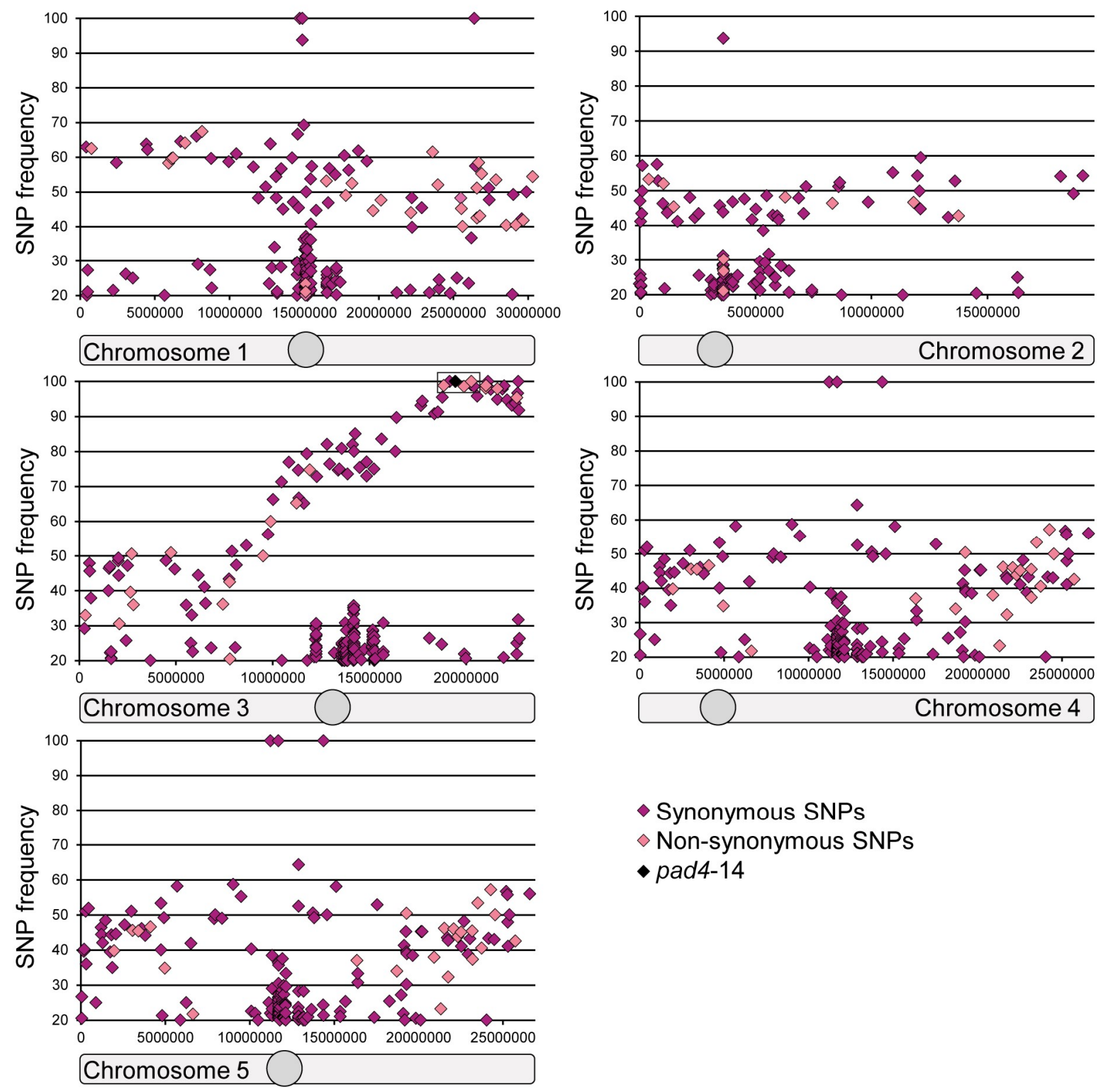

Figure 41: The suppressor mutation in noce12-23 is located on the lower arm of chromosome three. $150 \mathrm{bp}$ paired end Illumina sequencing reads were aligned to the TAIR10 reference genome using CLC genomics workbench 10.1.1. noce 12-23 suppressor SNP frequencies were mapped to their position on the chromosomes. Parameters for aligning and SNP call: Length fraction: 0.85; Similarity fraction: 0.8; minimum coverage $=10$, minimum counts $=2$, minimum frequency $=20 \%$; minimum central quality $=20$; minimum neighborhood quality $=15$. Duplicate SNPs present in cerk1-4, noce1-4, noce9-1 or noce8-1 were subtracted. Synonymous SNPs are shown as purple diamonds, SNPs causing non-synonymous mutations are depicted as pale pink diamonds. Grey boxes below the graphs symbolize chromosomes with circles marking the centromeres. The black box highlights the region containing the suppressor mutation. The best candidate, pad4-14, is depicted with a black diamond.

The best candidates for the suppressor mutation with SNP frequencies of at least $98 \%$ within the peak on chromosome three are shown in table 19. Among these candidates one mutation was identified causing an amino acid exchange in the EDS1-PAD4 (EP) domain of phytoalexin-deficient 4 (PAD4). PAD4, a lipase-like gene, is known to form a complex with en- 
hanced disease susceptibility 1 (EDS1) to promote accumulation of SA (Feys et al., 2001; Jirage et al., 1999; Wagner et al., 2013). The cerkl-4 cell death phenotype is SA-dependent. Accordingly, cerkl-4 cell death is abolished in the cerk1-4 pad4-1 double mutant (Petutschnig et al., 2014). This makes it most likely that the identified mutation in PAD4 is the suppressor mutation in noce12-23. This novel pad4 mutation was tentatively named pad4-14 as 13 other pad4 mutant alleles were described before (Neubauer et al., 2020).

Table 19: Suppressor candidates in noce 12-23.

\begin{tabular}{|c|c|c|c|c|c|c|}
\hline $\begin{array}{l}\text { Reference } \\
\text { Position }\end{array}$ & SNP & Frequency & $\begin{array}{l}\text { Overlapping } \\
\text { annotations }\end{array}$ & $\begin{array}{l}\text { Coding region } \\
\text { change }\end{array}$ & $\begin{array}{l}\text { Amino acid } \\
\text { change }\end{array}$ & Annotation ${ }^{\mathrm{f}}$ \\
\hline 18846445 & $\mathrm{C}>\mathrm{T}$ & 98.8 & AT3G50710 & $229 \mathrm{G}>\mathrm{A}$ & Glu77Lys & $\begin{array}{l}\text { F-box/RNI-like/FBD-like do- } \\
\text { mains-containing protein }\end{array}$ \\
\hline 19434162 & $\mathrm{C}>\mathrm{T}$ & 100 & $\begin{array}{l}\text { AT3G52430 } \\
\text { PAD4 }\end{array}$ & $1496 \mathrm{C}>\mathrm{T}$ & Ser499Phe & $\begin{array}{l}\text { lipase-like gene important for } \\
\text { SA signaling }\end{array}$ \\
\hline 19446887 & $\mathrm{C}>\mathrm{T}$ & 100 & AT3G52460 & & & $\begin{array}{l}\text { hydroxyproline-rich glycopro- } \\
\text { tein family protein }\end{array}$ \\
\hline 19897163 & $\mathrm{C}>\mathrm{T}$ & 98.5 & AT3G53680 & $250 \mathrm{G}>\mathrm{A}$ & Gly84Ser & Acyl-CoA N-acyltransferase \\
\hline 20265448 & $\mathrm{C}>\mathrm{T}$ & 100 & AT3G54750 & $\begin{array}{l}1357 \mathrm{G}>\mathrm{A}^{\mathrm{a}} \\
1354 \mathrm{G}>\mathrm{A}^{\mathrm{b}}\end{array}$ & $\begin{array}{l}\text { Asp453Asn }{ }^{\mathrm{a}} \\
\text { Asp452Asn }\end{array}$ & \\
\hline 20272165 & $\mathrm{C}>\mathrm{T}$ & 99.0 & AT3G54760 & & & $\begin{array}{l}\text { dentin sialophosphoprotein-like } \\
\text { protein }\end{array}$ \\
\hline 20413481 & $\mathrm{C}>\mathrm{T}$ & 98.6 & AT3G55080 & $\begin{array}{l}628+1 G>A^{c} \\
376+1 G>A^{d} \\
283+1 G>A^{e}\end{array}$ & & SET domain-containing protein \\
\hline
\end{tabular}

${ }^{a}$ position refers to splicing variant three, four and five.

${ }^{b}$ position refers to splicing variant one and two.

c position refers to splicing variant one.

d position refers to splicing variant three and five.

e position refers to splicing variant two and four.

f www.arabidopsis.org

The mutation identified in pad4-14 is an exchange of cytosine ${ }^{1496}$ to thymine that results in an amino acid exchange of serine ${ }^{499}$ to phenylalanine (Figure 42A). In general, the exchange of a polar neutral amino acid to a non-polar aromatic amino acid might interfere with protein structure and stability and therefore with protein function. The serine residue mutated in pad4-14 is conserved in another lipase-like gene, SAG101, but not in EDS1 (Figure 42B). The structure of the SAG101-EDS1 heterodimeric complex was crystallized and a very similar structure was modelled for the PAD4-EDS1 complex (Wagner et al., 2013). Serine ${ }^{499}$ in PAD4 is äquivalent to serine ${ }^{493}$ in SAG101 and this mutation is localted close to one of three $\alpha$-helices that are forming an interface for interaction with other proteins (Figure S9). There- 
fore, this interface might be altered in PAD4-14. As it was known already that PAD4 is necessary for the cerk1-4 cell death phenotype, no further experiments were conducted with pad4-14 in this work.

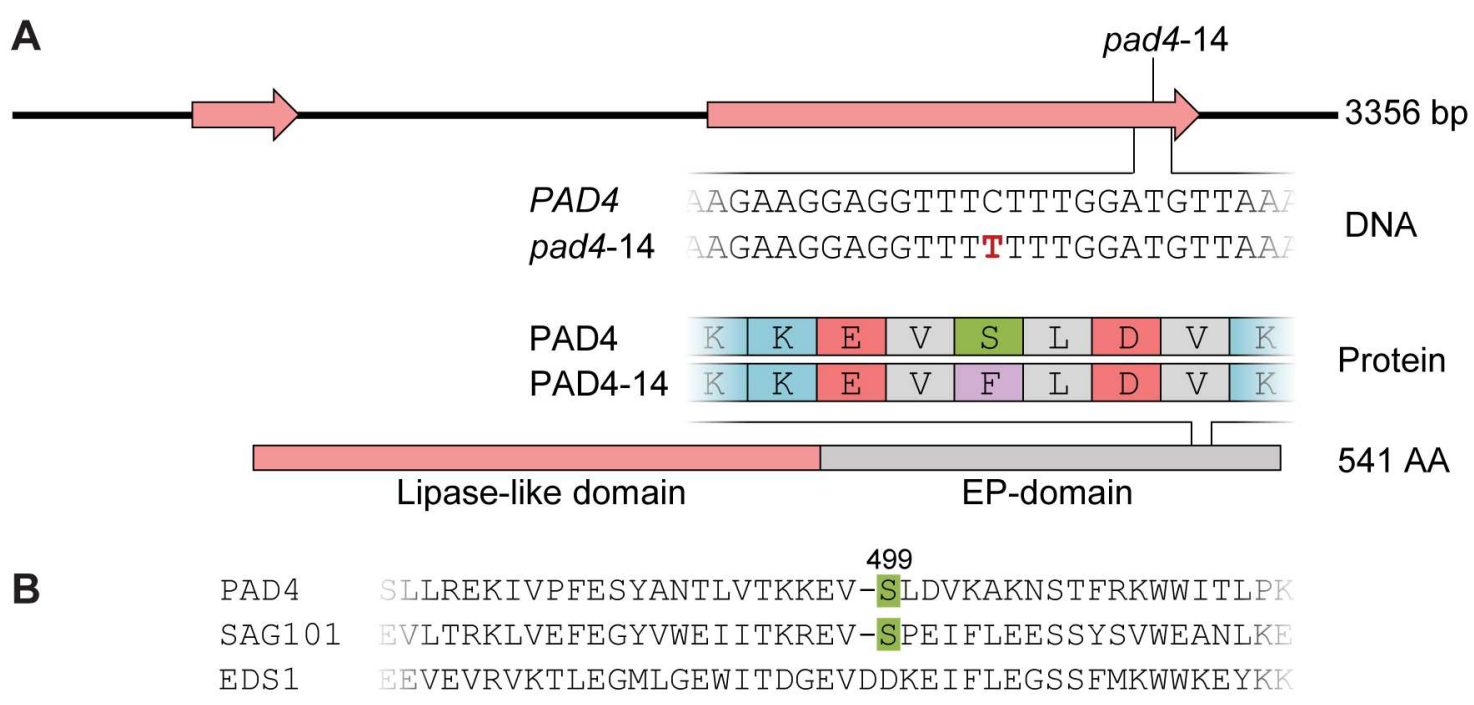

Figure 42: The pad4-14 mutation identified in the cerk1-4 suppressor screen.

A: Schematic structures of the PAD4 gene and protein are shown. Exons are depicted as pale pink arrows. The DNA sequence alignment shows the cytosine ${ }^{1496}$ to thymine nucleotide exchange (red), the protein alignment the serine ${ }^{499}$ to phenylalanine exchange in pad4-14. Protein domains were annotated according to Wagner et al., 2013. Color code for amino acids: Polar positive= blue, polar negative $=$ red, polar neutral $=$ green, non-polar aliphatic $=$ grey, non-polar aromatic $=$ purple. $\mathbf{B}:$ A section of an alignment of the lipase-like proteins PAD4, SAG101 and EDS1generated with clustal omega (Madeira et al., 2019). The conserved serine residue is highlighted in green and the number above the residue refers to the amino acid position in PAD4. 


\section{Discussion}

CERK1, a major component of the Arabidopsis chitin receptor (Miya et al., 2007), is modified by ectodomain shedding (Petutschnig et al., 2014). The ectodomain fragment is not present in cerk 1-4 mutants carrying a single amino acid exchange of leucine ${ }^{124}$ to phenylalanine. However, the ectodomain is most likely still cleaved in this mutant since C-terminal fragments are detectable, but it appears to be either unstable or targeted for degradation (Petutschnig et al., 2014). Moreover, cerkl-4 plants show a deregulated SA-dependent cell death response upon infection with powdery mildews and in senescent plants. This cell death is mediated by the CERK1-4 ectodomain and transmembrane domain and CERK1 kinase activity is not required (Petutschnig et al., 2014). Chitin-induced defense-responses are WTlike in cerk $1-4$ plants suggesting the deregulated cell death response to be independent from the function of CERK1 in PAMP signaling (Petutschnig et al., 2014). It is still unknown, how the ectodomain is shed, how high SA levels are accumulated in cerk1-4 plants and how cell death is induced.

CERK1 is a key component of the chitin receptor and chitin-induced immune responses are completely abolished in the cerkl-2 knockout mutant. Therefore it would make sense for the plant to monitor the integrity of this receptor and to induce cell death if CERK1 is not intact anymore.

For complexes containing BAK1, a co-receptor of multiple RLKs involved in the regulation of a number of various processes, such a surveillance system was proposed (Gao et al., 2009; Halter et al., 2014; Gao et al., 2018). BAK1 interacts with BAK1-INTERACTING RECEPTOR-LIKE KINASE 1 (BIR1) and birl mutants display a constitutive cell death response (Gao et al., 2009). It has been proposed that the BAK1/ BIR1 complex is guarded by two or more $\mathrm{R}$ proteins and loss of BAK1 or BIR1 function triggers the activation of these R proteins in the absence of pathogens (Gao et al., 2009; Gao et al., 2018)

Interestingly, not only knockout but also overexpression of BAK1 results in spontaneous cell death, suggesting that BAK1 protein level appears to be critical for cell death control (Dominguez-Ferreras et al., 2015). Moreover it was shown that BAK1 is C-terminally cleaved upon MAMP treatment within or close to the transmembrane domain. BAK1 mutant versions that can no longer be cleaved are unable to mediate PAMP signal transduction but still elicit cell death when overexpressed (Zhou et al., 2019). These findings indicate that BAK1 overexpression-induced cell death is not simply due to increased PAMP signaling but 
additional mechanisms must be in place. Up to now it is not clear if the proposed BAK1 surveillance system(s) sense full length BAK1 or the C-terminal cleavage product.

Possibly, CERK1 integrity monitoring might be mediated by the CERK1 ectodomain. A yet unidentified receptor could bind the CERK1 ectodomain in the WT situation and possibly induces cell death in cerkl-4 plants since the ectodomain is absent. However, cerkl-2 TDNA insertion mutants do not show cell death, but cell death can be induced in cerkl-2 by expression of cerk1-4 (Petutschnig et al., 2014), which argues against this hypothesis. The TDNA insertion in cerkl-2 is located in intron ten and CERK1 transcript upstream of the TDNA insertion is present (Miya et al., 2007). Small amounts of CERK1 ectodomain were detected in cerk1-2 Western Blots (Petutschnig et al., 2014), suggesting that this reduced amount of CERK1 ectodomain in cerk1-2 might be sufficient for receptor monitoring.

Another working model for cerkl-4 cell death induction is that turnover products of the cerk1-4 ectodomain or alterations in full length CERK1-4 could be recognized as DAMP molecules by a yet unknown receptor. BAK1 and SOBIR1 are co-receptors of many RLKs and RLPs (Gust and Felix, 2014; Ma et al., 2016) therefore it was tested if cerkl-4 cell death is BAK1 or SOBIR1 dependent. For this, cerk1-4 was crossed with bakl and sobirl mutants (E. Petutschnig, unpublished). In both cerk1-4 bak1 and cerk1-4 sobirl double mutants cell death was fully established (E. Petutschnig, unpublished), suggesting that the cerkl-4 cell death reaction is independent from BAK1 and SOBIR1. However, downstream signaling might be mediated by other co-receptors.

Upon DAMP perception, immune responses including the accumulation of reactive oxygen species are induced (Choi and Klessig, 2016). To investigate if ROS production by the NADPH oxidase RBOHD is necessary to establish the cerk1-4 cell death phenotype, cerk 1-4 rbohd mutants were generated (E. Petutschnig, unpublished). These mutants did not show a ROS burst upon flg22 treatment, but developed the cerkl-4 cell death phenotype. Therefore the exaggerated cell death response appears to be independent from ROS production, which would be unusual for a DAMP-induced immune response.

To identify molecular components necessary for cerkl-4 cell death induction, a forwardgenetic suppressor screen was started. In this screen, XLG2 (M. Stolze, C. Meusel), CRK7 and CRK43 (this work) were identified as proteins involved in cerk1-4 cell death formation so far. 


\section{$\underline{4.1 \text { CRK7 }}$}

\subsection{1 crk7 mutants show semi-dominant phenotypes}

A single amino acid exchange of cysteine ${ }^{94}$ to tyrosine in CRK7 was identified as the causative cerk1-4 suppressor mutation in noce9-1 (3.1.1). Structural information is available for the single DUF26 domain containing protein GNK2 from Ginkgo biloba (Miyakawa et al., 2009) and for the Arabidopsis double DUF26 domain containing proteins PDLP5 and PDLP8 (Vaattovaara et al., 2019). These studies showed that DUF26 domains have a highly conserved structure with two $\alpha$-helices that are folding on top of a five-stranded antiparallel $\beta$ sheet (Figure 2). Strands one, three and five of the $\beta$-sheet are covalently linked to the second $\alpha$-helix by three disulfide bridges (Miyakawa et al., 2009; Vaattovaara et al., 2019).

Most Arabidopsis CRKs, including CRK7, contain two DUF26 domains. In the first DUF26 domain all cysteine residues that are necessary for disulfide bridge formation in GNK2 and PDLP5/8 (Miyakawa et al., 2009; Vaattovaara et al., 2019) are conserved in AtCRKs (Figure S1A). Interestingly, the second DUF26 domain is less conserved since one or two cysteines crucial for disulfide bridge formation in PDLP5 (Vaattovaara et al., 2019) are missing in Arabidopsis CRKs (Figure S1B). This suggests that the second DUF26 domain of most Arabidopsis CRKs, including CRK7, contains only two disulfide bridges, which might result in an altered domain structure.

In the identified cerk1-4 suppressor mutant noce9-1, named crk7-4, the second highly conserved cysteine residue of the $\mathrm{C}-\mathrm{X}_{8}-\mathrm{C}-\mathrm{X}_{2}-\mathrm{C}$ motif in the first DUF26 domain (Figure S1A) of CRK7 is mutated to tyrosine (3.1.1). This suggests that the disulfide bridge connecting the third $\beta$-strand with the $\alpha$-helix in the first DUF26 domain is missing in the CRK7-4 protein, which might results in a larger distance between the $\alpha$-helix and the $\beta$-sheet. It was shown that the conserved cysteine residues are important for structure of DUF26 domain containing proteins since PDLP5 proteins in which three conserved cysteines were mutated to alanines formed aggregates in biochemical preparations and displayed reduced structural stability (Vaattovaara et al., 2019). The conserved cysteine residues were also shown to be important for protein function of CRK28 and CRK36. CRK28 induces a strong cell death response in $N$. benthamiana. crk28 mutant versions with single conserved cysteine residues exchanged to alanine, were detectable in Western Blots, suggesting that the mutated protein version was present, but failed to induce cell death in N. benthamiana (Yadeta et al., 2017). Moreover it was shown that the conserved cysteine residues in CRK36 are required for BIK1 phosphorylation and for the induction of immune responses after pathogen infection or flg22 treatment. 
Interestingly, CRK36 cysteine-mutants were still able to interact with BIK1, but unable to induce BIK1 phosphorylation (Lee et al., 2017), suggesting that mutation of conserved cysteines in the DUF26 domain can have an impact on kinase activity of the protein, possibly due to an overall altered protein structure.

Taken together, the mutation of a conserved cysteine residue in CRK7-4 possibly has an impact on CRK7-4 protein structure and mutated proteins cannot fulfill the CRK7 function in cerk1-4 cell death induction.

Another $c r k 7$ mutant obtained from the screen, $c r k 7-6$, is a truncated protein that lacks part of the kinase domain (3.1.2.2). Due to the lack of an $\alpha$-CRK7 antibody, it is not clear, if the truncated CRK7-6 protein is stable. If it is stable, is might be still able to interact with a potential ligand, but most likely unable to further transduce the signal since the truncated kinase domain is not functional.

Besides the $c r k 7$ mutants from the screen, also the T-DNA mutant line $c r k 7-5$ was investigated in this study (3.1.2.1). The T-DNA insertion in this line is localized in exon one before the first conserved $\mathrm{C}-\mathrm{X}_{8}-\mathrm{C}-\mathrm{X}_{2}-\mathrm{C}$ motif, thus a possible truncated protein would be likely nonfunctional. Additionally, a reduced $C R K 7$ transcript downstream of the T-DNA insertion was detected in $c r k 7-5$. If this is translated, the resulting protein would lack the signal peptide, leading to miss-localization.

To obtain a clear $c r k 7$ knockout line, the whole $C R K 7$ gene was deleted using the CRISPR/ Cas9 system and the generated mutant was named crk7-7 (3.1.2.3).

Interestingly, all crk7 mutants showed a semi-dominant cell death phenotype upon infection with E. cruciferarum. Semi-dominant phenotypes can be in general caused by mutated protein versions that still fulfill part of the protein functions or by gain of function mutations leading to new protein functions or altered gene expression. Moreover dominant negative mutations, whose gene products negatively affect the wildtype gene product, can lead to semi-dominant phenotypes. Often also gene dose effects play an important role for semidominance (Wilkie, 2006).

In crk7-4 a single amino acid is exchanged and in $c r k 7-6$ a truncated protein might be present. These mutated protein versions could still fulfill part of the CRK7 protein function or might have dominant-negative effects since they are potentially unable to bind the unknown substrate (crk7-4) or might be impaired in signal transduction (crk7-6) respectively. In crk7-5 a dominant negative effect is rather unlikely since a possible truncated protein would be very short and likely non-functional and a potential gene product 5' of the T-DNA insertion is sup- 
posed to be miss-localized as it lacks the signal peptide. Thus, the semi-dominant phenotype might be caused by a gene-dose effect of $C R K 7$.

Since the same phenotype was also observed in $c r k 7-7$, a mutant completely lacking the CRK7 coding region, semi-dominance cannot be explained by gain of function or dominant negative effects.

Taken together, a $C R K 7$ gene dose effect is the most likely explanation for the observed semi-dominant phenotypes in $c r k 7$ mutants.

In another study CRK7 was named Staphylococcus elicitor response 1 (STER1) (Dinischiotu, 2011). T-DNA insertion lines were investigated in this study and one of these lines (ster 1-1) was semi-dominant whereas the two other T-DNA lines (ster1-4, ster1-5) appeared to be recessive. The T-DNA insertion in ster $1-1$ is located in intron two, but the T-DNA insertions in ster 1-4 and ster 1-5 are at the end of the last CRK7 exon and in the 3' UTR respectively. Since $C R K 8$ is encoded about 1000 bp downstream of $C R K 7$, not only $C R K 7$ but also $C R K 8$ expression might be altered in ster 1-4 and especially in ster $1-5$ mutants. CRK7 and CRK8 are closely related CRKs and might fulfill redundant or partially redundant functions which potentially explains the recessive phenotypes in ster $1-4$ and ster $1-5$. Similar to the observations in this study, the semi-dominant phenotype observed in sterl-1 was suggested to be the result of a dosage effect (Dinischiotu, 2011).

In rice, a single amino acid exchange in a CRK named APOPTOSIS LEAF AND SHEATH 1 (ALS1) or LIGHT-INDUCED LESION MIMIC MUTANT 1 (LIL1), led to strong overexpression of ALS1/ LIL1 resulting in enhanced cell death formation (Zhou et al., 2017; Du et al., 2019). This observed phenotype was also semi-dominant and a dose-dependence effect was proposed (Zhou et al., 2017; Du et al., 2019).

Therefore, dosage dependency of CRKs might be a more common mechanism.

\subsubsection{PAMP responses are not altered in crk7 mutants}

Several CRKs were shown to be involved in PAMP triggered immunity including ROS burst, MAPK activation and defense gene expression (Yeh et al., 2015; Lee et al., 2017; Yadeta et al., 2017; Kimura et al., 2020). One of the closest homologues of CRK7, CRK6 (Bourdais et $a l ., 2015)$, interacts with FLS2 and is involved in flg22-induced stomatal closure and defense gene expression (Yeh et al., 2015). Overexpression of CRK6 resulted in enhanced resistance towards Pseudomonas syringae infection whereas knockout of CRK6 had no effect on resistance against Pst, probably due to functional redundancy between closely related CRKs 
(Yeh et al., 2015). CRK7 was identified as a molecular component involved in cerk1-4 cell death formation, a mechanism independent from the CERK1 function in chitin-triggered immunity.

In this study it was investigated if CRK7 is involved not only in cerk1-4 cell death but also in PAMP responses upon flg22 or chitin treatment (3.1.3).

The investigated $c r k 7-4$ and $c r k 7-5$ mutant lines were neither impaired in chitin nor flg22 triggered ROS accumulation (3.1.3.1), activation of MAPKs (3.1.3.2) or defense gene expression (3.1.3.3). This suggests that either CRK7 does not play a role in these PAMP-triggered responses or the function of CRK7 in PTI is masked due to redundancy with closely related CRKs. Previously it was shown that most crk knockout lines lack strong phenotypes (Bourdais et al., 2015). In contrast, many investigated overexpression lines often had clear phenotypes (Acharya et al., 2007; Yeh et al., 2015; Lee et al., 2017; Yadeta et al., 2017). Therefore, it would be interesting to investigate the PAMP responses in CRK7 overexpressing lines to figure out if CRK7 is involved in PTI additional to its function in cell death induction. Since overexpression of CRK7 above a certain threshold leads to silencing of both native and transgenic $C R K 7$ (4.1.6), no stable overexpressing lines could be generated and hence PTI responses in a CRK7 overexpression background cannot be investigated.

\subsubsection{CRK7 localizes predominantly to the plasma membrane and CRK7-4 is restricted to the}

$\underline{\text { ER }}$

In this work, the localization of CRK7 and the mutant version CRK7-4, fused to Citrine and mCitrine fluorescence tags, was investigated upon transient expression in $N$. benthamiana and stable expression in Arabidopsis (3.1.5). In both N. benthamiana and Arabidopsis CRK7Citrine localized to the plasma membrane, the ER and the nuclear envelope. Additionally, in cells with stronger fluorescence, very bright round structures were observed. These structures are highly similar in appearance to organized smooth ER (OSER) structures described in plants (Ferrero et al., 2015). OSER is a collective term for differently shaped highly ordered ER membranes and OSER formation has been observed under physiological conditions as well as during drug treatments in different organisms (Snapp et al., 2003; Almsherqi et al., 2009). These naturally formed OSER are suggested to fulfill specific biosynthetic and secretory functions (Ferrero et al., 2015). Additionally, OSER formation was observed upon overexpression of ER resident transmembrane proteins in yeast, mammalian and plant cells (Snapp et al., 2003; Ferrero et al., 2015). 
One model for OSER formation is that the cytoplasmic domains of overexpressed ERmembrane proteins localized next to each other interact and therefore "zip" the membranes together (Snapp et al., 2003). In some cases, interaction of the ER resident protein at the Nas well as C-terminus may be necessary for OSER development. One example for such a "two-lock mechanism" is Langerin, which interacts via its N-terminal domain and causes OSER in human cells only when tagged with a C-terminal YFP, whereas monomeric or Nterminal tags on Langerin do not lead to OSER formation (Lenormand et al., 2013). Therefore, addition of fluorescence tags to secreted proteins can lead to OSER formation and in this case OSERs might not represent native protein localization.

In this study, at first localization of Citrine-tagged CRK7/ CRK7-4 proteins was examined. The Citrine tag is able to form weak dimers (Cranfill et al., 2016), but dimerization of the Citrine tag alone cannot be the reason for OSER formation since CRK7-4-Citrine proteins did not show any OSER-like structures.

In contrast to Citrine, mCitrine is unable to dimerize (Cranfill et al., 2016). CRK7-mCitrine proteins localized predominantly at the plasma membrane and only in some cells with higher fluorescence, ER and nuclear envelope localization was observed additionally (3.1.5.2.2). In a low number of cells that expressed CRK7-mCitrine at a very high level, angular aggregates were present. However, these were smaller and less bright than the round structures observed with CRK7-Citrine. Since OSER can be structurally highly different, these angularly shaped aggregates may represent another type of OSER structures. These results indicate that strong overexpression of CRK7 itself appears to be sufficient to induce OSER formation, but the Citrine tag clearly promotes this, possibly through stacking of the ER-membranes according to the "Two-lock mechanism" proposed by Lenormand and colleagues (2013). The observed aggregates are most likely due to CRK7 overexpression and possibly do not represent native CRK7 localization. To confirm the structures observed in CRK7 overexpressing plants as OSERs, one could perform transmission electron microscopy, since OSER structures can be clearly recognized in plant cells by transmission EM (Ferrero et al., 2015).

Interestingly, CRK7-4-Citrine and CRK7-4-mCitrine localized exclusively to the ER independently of signal strength and did not show any OSER-like structures in $N$. benthamiana or Arabidopsis (3.1.5).

According to the two-lock mechanism, OSER are formed by interaction of the N-terminal domain in the ER-lumen and interaction of the fluorescent protein in the cytoplasm (Lenormand et al., 2013). The lack of OSER in CRK7-4-(m)Citrine expressing cells indicates that an intact DUF26 domain is necessary for homomerization and therefore OSER formation. 
Indeed, Co-IP experiments revealed that CRK7-4 was unable to homodimerize (3.1.6.2; 4.1.4), suggesting that this is the reason for the lack of OSER.

The exclusive ER-localization of CRK7-4 is likely caused by retention through the ER quality control system. In proteomics experiments it was shown that CRK7-4 interacts with chaperones such as ER luminal-binding proteins (BiPs; 3.1.6.3.2) in N. benthamiana to a much greater extent than CRK7. Preliminary results confirm the interaction with similar proteins in Arabidopsis (Figure S6).

Mutations in amino acids important for protein structure, like in CRK7-4, can prevent proper protein folding. Plants have developed diverse ER quality control mechanisms to prohibit that misfolded proteins proceed further along the secretory pathway (Howell, 2013). The chaperones calnexin and calreticulin are involved in ER quality control and monitor the efficient folding of glycoproteins whereas BiPs prevent protein aggregation in the ER (Braakmann and Hebert, 2013). If a protein cannot be properly folded and/ or aggregates, it is often exported and degraded via the ER-associated protein degradation (ERAD) pathway (Otero et al., 2010; Howell et al., 2013 Braackman et al., 2013).

Since CRK7-4 has an amino acid exchange in a conserved and structurally important cysteine residue (3.1.1) it is likely that CRK7-4 is not properly folded and therefore recognized by the ER quality control mechanism. The observed fluorescence signal in CRK7-4 samples was usually lower than the signal observed for WT CRK7. This suggests that CRK7-4 might be exported after recognition as a misfolded protein and degraded rapidly.

ER-retention was also demonstrated for two bril mutants. The brassinosteroid receptor BRI1 mainly localizes to the plasma membrane, but two BRI1 mutant versions with single amino acid exchanges (bri1-5 and bri1-9) accumulate predominantly in the ER. Consequently, the mutant plants show a brassinosteroid-deficient dwarf phenotype (Hong et al., 2008; Su et al., 2010). The observed dwarf phenotype was shown to be not only caused by bril-5 and bril-9 ER retention, but also by ERAD, since inhibition of the ERAD system led to partial suppression of the dwarf phenotype in bril-5 and bril-9 (Hong et al., 2008; Su et al., 2010).

To test if the cerk1-4 crk7-4 suppressor phenotype is due to the ER-retention of misfolded CRK7-4 protein, it might be interesting to investigate CRK7-4 localization and mutant phenotypes in lines impaired in ERAD. Nevertheless, since CRK7-4 proteins were unable to form homomers (4.1.4), it is rather unlikely that ER-retention is the only reason for the nonfunctionality of CRK7-4 in cell death induction. 


\subsubsection{CRK7 is able to form oligomers in planta}

In Western Blot analysis additionally to the band that fits the monomeric glycosylated CRK7/ CRK7-4 protein, a higher molecular weight band was detected in CRK7 samples (3.1.6.1). This band was present in mCitrine, Citrine and FLAG tagged CRK7 samples, suggesting that it is an intrinsic feature of CRK7 and independent of the tag.

Since the high molecular weight band shows a similar intensity to the monomeric signal and the interaction is very stable, potential interacting proteins were expected to be co-isolated with CRK7 in large amounts. In contrast, in CRK7-4 samples the high-molecular weight band was far less abundant and therefore such interacting proteins should be pulled down with CRK7-4 only in small quantities. However, proteomics experiments did not yield any candidate proteins matching these criteria. Consequently, the high molecular weight bands most likely represent complexes that contain exclusively CRK7/ CRK7-4.

In CRK7-Citrine and CRK7-mCitrine samples only one high molecular weight band with a size of about $250 \mathrm{kDa}$ was detectable. In contrast, in CRK7-FLAG samples, three high molecular weight bands of about $280 \mathrm{kDa}, 215 \mathrm{kDa}$ and $200 \mathrm{kDa}$ were present. Since the (m)Citrine tag alone has a size of $27 \mathrm{kDa}$ and the FLAG tag is much smaller with only $3 \mathrm{kDa}$, most probably the different high molecular weight bands present in the CRK7-FLAG samples were not detectable in SDS-PAGE for CRK7-(m)Citrine samples due to the overall larger protein size. The $250 \mathrm{kDa}$ band in the CRK7-(m)Citrine samples and the $200 \mathrm{kDa}$ band in the CRK7-FLAG sample fit the expected size of glycosylated CRK7 dimers. The $280 \mathrm{kDa}$ band observed in the CRK7-FLAG Western Blots nearly fits the size of a CRK7-trimer and the $215 \mathrm{kDa}$ band might represent a truncated trimer. Truncation of the trimer might be a process only happening in vitro and possibly does not represent a native situation. However, since distinct bands are present, it is suggested that the aggregate formation is specific.

CRK7 dimerization is most likely not only the reason for the higher molecular weight band observed in Western Blots but also necessary for OSER formation observed in CLSM experiments according to the "two-lock mechanism" (Lenormand et al., 2013) as explained before (3.1.5; 4.1.3).

In general it is remarkable that the proposed CRK7 dimers/ oligomers are visible in Western Blots since proteins are usually denatured and therefore protein interactions are destroyed by boiling the samples in SDS loading dye. Since all cysteine residues within one DUF26 domain are connected via intramolecular disulfide bridges, the formation of intermolecular disulfide bridges between two CRK7 proteins is rather unlikely. Moreover, disulfide bridges 
should be broken by reduction with DTT present in the SDS sample buffer. Therefore, the reason why these high molecular weight bands are so stable, remains elusive.

In CRK7-4 samples the high molecular weight band was far less abundant than in CRK7 samples (3.1.6.1), suggesting that the interaction responsible for the formation of this band is reduced in CRK7-4. This indicates that the first DUF26 domain of CRK7 is required for complex formation, since it carries a mutation in CRK7-4 and probably does not have the correct three-dimensional structure.

Co-IP experiments were conducted to investigate if CRK7 is truly able to dimerize (or form higher order homotypic oligomers) and if this ability is impaired in CRK7-4. For this, CRK7mCitrine was co-expressed with CRK7-FLAG and CRK7-4-mCitrine was co-expressed with CRK7-4-FLAG in N. benthamiana. The protein extracts were used for FLAG as well as GFP pull-downs, followed by $\alpha$-GFP and $\alpha$-FLAG Western Blots (3.1.6.2). This experiment revealed that CRK7-FLAG was pulled down with CRK7-mCitrine and vice versa, but CRK7-4FLAG was not pulled down with CRK7-4-mCitrine or the other way around. Therefore, the ability of CRK7 to form dimers/ oligomers was confirmed, suggesting that the high molecular weight bands observed in CRK7 Western Blots are due to dimerization/ oligomerization.

Both, the high molecular weight band in Western Blots and OSER formation, were observed in N. benthamiana and Arabidopsis plants expressing CRK7-(m)Citrine, suggesting that CRK7 homodimerization (or oligomerization) takes place in both plant species. CRK7 appears to dimerize either in a ligand-independent manner or upon perception of a ligand present in both $N$. benthamiana and Arabidopsis. Since cerkl-4 plants do not only show cell death upon infection with adapted and non-adapted powdery mildews but also in senescent plants (Petutschnig et al., 2014), possibly an endogenous ligand leads to cell death induction. Homodimerization was also reported for CRK28 and CRK36, but in contrast to CRK7 no high molecular weight band was observed in Western blots (Tanaka et al., 2012; Yadeta et al., 2017). Additionally, CRK28 was shown to interact with CRK29, BAK1 and FLS2 in a ligand-independent manner in $N$. benthamiana (Yadeta et al., 2017). CRK36 furthermore associates with CRK45 and FLS2 independently of flg22 elicitation and was shown to directly phosphorylate BIK1 after flg22 treatment (Tanaka et al., 2012; Yeh et al., 2015; Lee et al., 2017). These results suggest that ligand-independent homo- or heterodimerization of CRKs might be a more common mechanism.

To further investigate CRK7 dimerization in planta, FLIM-FRET analyses with CRK7mCitrine and CRK7-mScarlet-I will be conducted in the future. 


\subsubsection{CRK7 is an active kinase}

Idänheimo and colleagues (2014) showed that the CRK7 cytoplasmic domain expressed in $E$. coli has autophosphorylation as well as transphosphorylation activity. These findings could be confirmed in kinase assays with full-length CRK7 protein purified from $N$. benthamiana in this work (3.1.6.4).

In proteomics experiments, CRK7-mCitrine proteins extracted from transiently transformed N. benthamiana (3.1.6.3.1) and stably transformed Arabidopsis plants (Table S2) were highly phosphorylated at several residues in the activation and $\mathrm{P}+1$ loop of the kinase domain, whereas phosphorylation of these domains was substantially reduced in CRK7-4-mCitrine. Many kinases are activated by phosphorylation in their kinase subdomains, since phosphorylation of the activation loop leads to large conformational changes that allow substrate binding and catalysis (Taylor and Radzio-Andzelm, 1994; Huse and Kuriyan, 2002). Therefore it is conceivable that CRK7 may be activated in planta by dimerization/ oligomerization and that activation may be reduced in CRK7-4. When used in in vitro kinase assays, CRK7-4 isolated from $N$. benthamiana still displayed kinase activity (3.1.6.4). This suggests that either the observed difference in phosphorylation does not impact enzymatic performance, or that phosphorylation takes place in the in vitro assay. To investigate this hypothesis further, mutated versions of CRK7 were generated, in which the phosphorylatable amino acids in the activation and/ or the $\mathrm{P}+1$ loop were exchanged for non-phosphorylatable residues (3.1.6.4). Kinase assays revealed that mutations in the activation loop alone did not reproducibly alter kinase activity, while CRK7 protein with mutations in both the activation and $\mathrm{P}+1$ loop was kinase dead. These findings support the initial hypothesis that CRK7-4 may be less active than CRK7 in planta. Other differentially phosphorylated sites could not be investigated in this study due to time limitations and should be addressed in future work.

To investigate if CRK7 kinase activity is required for cerkl-4 cell death induction, it would be interesting to express the kinase dead CRK7 version in cerk1-4 crk7-4 plants. Since complementation experiments with semi-dominant $c r k 7$ mutant lines are rather difficult (3.1.3; 4.1.6), this experiment should be postponed until CRK7 complementation is established.

Taken together, it was shown that CRK7 is able to form homodimers (or oligomers), is an active kinase and highly phosphorylated at several residues in the activation and $\mathrm{P}+1$ loop. CRK7-4 is also kinase active in vitro, but phosphorylation at the activation and $\mathrm{P}+1$ loop is substantially reduced and dimerization is abolished. 


\subsubsection{CRK7 complementation approaches}

crk7-4 was confirmed as suppressor mutation by analysis of independent crk7 mutants (3.1.2). Since all investigated crk7 mutants showed semi-dominant phenotypes, likely due to dosis-dependency of CRK7 (4.1.1), complementation was rather difficult (3.1.4). Complementation experiments were conducted in two opposite approaches. In the first approach, CRK 7-4 was (over)expressed in cerk1-4. In this case, a dominant negative effect would result in a cerk1-4 suppressor phenotype. However, plants overexpressing CRK7-4 in cerk1-4 did not show any suppression of the cell death phenotype. Later in the course of the project, confocal microscopy revealed that CRK7-4 is retained in the ER, whereas the wild type CRK7 version is primarily located at the plasma membrane. Since CRK7-4 is mislocalized, it is unlikely to outcompete the native CRK7 at the PM. Additionally, CRK7-4 is unable to form homodimers/ -oligomers (4.1.4) suggesting that it is also functionally defective. Taken together, these findings explain why this approach was unsuccessful.

However, it has to be mentioned that some cerk1-4 plants transformed with p35S::crk7-4Citrine did show a suppressor phenotype. Confocal microscopy and Western blotting experiments revealed that these plants did not accumulate the transgenic protein, making gene silencing the most likely explanation. This notion is supported by the fact that a suppressor phenotype was also observed when the wild type CRK7-Citrine version was overexpressed in cerk 1-4. It is known that transgene expression from the $35 S$ promotor can lead to gene silencing, including co-suppression of the native and transgenic transcript (de Buck et al., 2013). Since overexpression of several CRKs was shown to induce cell death (Chen et al., 2004; Acharya et al., 2007; Yadeta et al., 2017, Lee et al., 2017) CRK expression above a certain threshold might lead to silencing to prevent unwanted cell death reactions.

In the second approach CRK7 was expressed in cerk1-4 crk7-4 and cerk1-4 crk7-5 plants. Here, complementation, i.e. re-establishment of the cerkl-4 cell death phenotype, was expected. Upon expression of CRK7 under the native promotor, complementation was unsuccessful, but CRK 7 overexpression under the $35 S$ promotor yielded about $30 \%$ transformants with cerk1-4 cell death phenotype. These results suggest that expression of CRK7 in noncomplementing lines either might not have reached the threshold level necessary for cell death induction, or CRK7 expression from the $35 \mathrm{~S}$ promoter may have led to silencing.

Surprisingly, none of the plants transformed with Citrine-tagged CRK7 re-established cell death. Since confocal microscopy and Western blotting experiments revealed that most of these plants did not accumulate the transgenic protein, gene silencing effects most likely con- 
tribute to this phenomenon. Nevertheless, in some plants strong expression of CRK7-Citrine was confirmed via confocal microscopy and Western blotting, but these plants did not show phenotypic complementation either. A possible explanation why these plants are unable to reestablish cell death, is CRK7-mCitrine OSER formation. As discussed before (4.1.3) OSER formation might be due to CRK7 overexpression and most probably does not represent native CRK7 localization. In cells in which CRK7 accumulated primarily in OSER structures, the amount of CRK7 left at the PM might be too low to reach the required threshold for cell death induction. Since less OSER formation was observed upon expression of CRK7mCitrine in $N$. benthamiana and Col-3 gll (3.1.5.2) it would be interesting to repeat complementation studies with this construct. However, Citrine is a large tag that might interfere with protein function. Therefore complementation experiments were also conducted by expression of CRK7-FLAG in cerk1-4 crk7-5. Expression of the transgene was confirmed via Western Blot at a low level and no phenotypic complementation was observed. Since it is not known, if the FLAG-tag is able to dimerize and therefore to support OSER formation, it is more likely that unsuccessful complementation in these lines was due to too low expression of the transgene.

Possibly the CRK7 complementation problem lies not with the transformed constructs or the properties of the CRK7 fusion proteins, but with the mutant backgrounds used. So far, complementation studies with CRK7 were conducted in cerk1-4 crk7-4 and cerk1-4 crk7-5. These lines carry mutated and truncated versions of CRK7, respectively, which could potentially interfere with complementation. The CRISPR/ Cas9 system was used to delete the entire CRK7 gene in cerk1-4 plants (3.1.2.3) and future complementation analyses should be conducted in this background.

Interestingly, the Arabidopsis ecotype Cvi-0 completely lacks the CRK7 gene (Dinischiotu, 2011). While Col-0 plants developed strong cell death responses upon S. epidermidis treatment, Cvi-0 plants were unable to induce cell death. This phenotype was fully complemented by expression of $p 35 S: \because C R K 7$ in Cvi-0. Moreover, Dinischiotu (2011) found that a crk7 TDNA insertion line in the Col-0 background was also insensitive to S. epidermidis. However, they faced similar problems as described in this study when attempting to complement this line. The described T-DNA mutant has a semi-dominant phenotype and expression of $p 35 S:: C R K 7$ conferred only a weak $S$. epidermidis cell death response to this line.

In summary, many questions are still open concerning the problems observed in CRK7 complementation studies. Nevertheless, it seems likely that the CRK7 protein amount is tightly 
regulated and has to reach a certain threshold for cell death induction, whereas overexpression results in OSER formation as well as gene silencing.

\section{$\underline{4.2 \text { CRK43 }}$}

\subsubsection{Confirmation of crk43-2 as cerkl-4 suppressor mutation}

In the forward genetic cerk1-4 suppressor screen an independent suppressor line was identified, which showed the same semi-dominant phenotype as observed for crk7 mutants. The identified suppressor mutation affects the gene encoding CRK43, another member of the CRK family (Bourdais et al., 2015). The mutation eliminates a spliceosome acceptor sequence leading to aberrant splicing and a potentially truncated protein (Figure 29). CRK43 is one of the three CRCKs in Arabidopsis without an ectodomain and the possibly resulting truncated protein CRK43-2 lacks more than half of the kinase domain.

Since the observed phenotype was semi-dominant, either the truncated CRK43-2 protein fulfills still a partial function in cell death induction, has a dominant-negative effect or the amount of CRK43 protein has to reach a threshold for the development of the full cell death phenotype, as discussed for CRK7 (4.1.6).

crk43-2 was confirmed as causative suppressor mutation by analysis of a T-DNA mutant line that mimics the semi-dominant phenotype upon E. cruciferarum infection. The T-DNA in this line is located in the first intron. A potential 5'prime transcript would be very short and would not contain any functional domains whereas a potential 3 'prime transcript would contain the entire kinase domain. If this 3 'transcript is translated, it could fulfill a partial function leading to semi-dominance.

Moreover, complementation experiments were conducted. For this purpose, untagged and mCitrine tagged CRK43 was expressed in cerk1-4 crk43-2 plants under the native promotor. Surprisingly, already a high number of uninfected plants transformed with untagged or tagged CRK43 showed a severe cell death and dwarf phenotype (3.2.2.2, Figure 33). Plants with normal development at the age of four weeks were inoculated with E. cruciferarum and showed a stronger cell death phenotype than cerk1-4 control plants (Figure 34). These results further confirmed crk43-2 as the cerkl-4 suppressor mutation and clearly indicate that CRK43 is involved in cell death formation.

Up to now, CRK43 was only expressed in the cerk1-4 crk43-2 background. Therefore it is not clear yet, if the observed cell death phenotype is dependent on cerk1-4 or may also occur in a CERK1 wild type background. However, preliminary data suggest that CRK43 can in- 
duce cell death also in $N$. benthamiana, which supports the idea that CRK43 can mediate cell death independently of cerk1-4.

To further investigate this, it would be interesting to transform $p C R K 43: \because C R K 43$ into Col-3 gll and to infiltrate $p 35 S:: C R K 43$ into $N$. benthamiana.

Previously it was reported that overexpression of CRK4, CRK5, CRK13, CRK19 and CRK20 leads to cell death formation in uninfected Arabidopsis plants (Chen et al., 2004; Acharya et al., 2007) and overexpression of CRK13, CRK28 and CRK29 induces cell death in N. benthamiana (Yadeta et al., 2017). Therefore, cell death induction by CRKs appears to be a more common mechanism.

RLCKs are typically associated with membrane-bound receptor complexes and act as downstream signal transducers (Liang and Zhou, 2018). Among the CRKs it was reported that CRK45, one of the three truncated CRCKs lacking the ectodomain, interacts with the fulllength receptor kinase CRK36 (Tanaka et al., 2012). Therefore, it would be possible that CRK43 interacts with CRK7 or another RLK necessary for cerk1-4 induced cell death for further signal transduction.

\subsubsection{PAMP responses are not altered in crk43 mutants}

Several CRKs were reported to play a role in PAMP-triggered immune responses (Yeh et al., 2015; Yadeta et al., 2017; Lee et al., 2017). One of them is CRK36, which also interacts with the truncated CRK45 (Tanka et al., 2012). Since overexpression of CRK45 leads to enhanced resistance to Pst and knockout results in higher susceptibility, CRK45 appears to be involved in disease resistance (Zhang et al., 2013b). This suggests that CRK45 is part of a receptor complex to mediate immune responses upon infection with bacterial pathogens. In general, CRK43 could be part of a similar receptor complex.

Therefore, it was examined if crk43 mutants are impaired in flg22 and chitin triggered immunity (3.2.3). The investigated crk43-2 and crk43-3 mutant lines were not impaired in CERK1 phosphorylation upon chitin infiltration (3.2.3.1) and showed WT-like ROS accumulation (3.2.3.2) as well as MAPK activation (3.2.3.3) upon flg22 and chitin treatment.

This suggests that CRK43 does not play a crucial role for the tested PAMP-triggered responses, similar to the observations for CRK7 (3.1.3; 4.1.2). Nevertheless, it cannot be excluded that the role of CRK43 in PAMP signaling might be masked by functional redundant $\mathrm{CR}(\mathrm{C}) \mathrm{Ks}$ in the crk43 mutant. However, given that CRK43 expression above a certain threshold results in cell death (3.2.4), analysis of overexpression lines does not seem feasible. 


\subsubsection{CRK43 localizes to the cell periphery, nucleus and nucleolus}

CRK43 is a CRCK and since no myristyolation sites or other lipid modifications are predicted (ARAMEMNON, Schwacke et al., 2003), cytosolic localization would be expected. Nevertheless, in the resting state most RLCKs interact with RLKs, resulting in enrichment at the plasma membrane. Upon ligand perception by the RLK, RLCKs become phosphorylated and dissociate from the complex for further signal transduction (Liang and Zhou, 2018). If CRK43 is also able to interact with plasma membrane localized RLKs, an enrichment of CRK43 fluorescence signal close to the plasma membrane would be expected.

The observed fluorescence signal of CRK43-mCitrine expressed from the CRK43 promoter in stably transformed Arabidopsis plants and transiently transformed N. benthamiana leaves was in general very low. This is in agreement with publicly available expression data (Winter et al., 2007). Since expression of $p C R K 43:: C R K 43-m C i t r i n e$ led to strong cell death in a large number of transformants, it can be assumed that even moderately or slightly higher CRK43 expression than in the wild type is harmful to the plant. A weak CRK43-mCitrine microscopy signal was present at the cell periphery as confirmed by co-localization studies with a plasma membrane marker in $N$. benthamiana and in cytoplasmic strands. To investigate if the fluorescence at the cell periphery is a plasma membrane associated signal, plasmolysis experiments should be conducted in the future. In addition to the observed florescence at the cell periphery, a more intense CRK43-mCitrine fluorescence signal was detected in the nucleus and especially in the nucleolus in $N$. benthamiana (3.2.4.1). In stably transformed Arabidopsis cerk1-4 crk43-2 plants weak CRK43-mCitrine fluorescence was detectable at the cell periphery and nuclear localization was observed in some cells. Nucleolar expression was not detected, which may be due to the overall very weak fluorescence. Nevertheless, the expression appeared to be slightly stronger upon E. cruciferarum infection than in uninfected plants. This is in line with the observed $C R K 43$ upregulation upon Bgh infection (Bourdais et al., 2015), but contrary to CRK43 downregulation upon Golovinomyces orontii infection shown in publically available microarray data (Chandran et al., 2010).

Sequence analysis of CRK43 predicted a potential N-terminal nuclear localization sequence (NLS) (cNLS mapper, Kosugi et al., 2009) and a C-terminal nucleolar localization sequence (NoLS) (Nucleolar localization sequence Detector (NoD), Scott et al., 2010).

Localization of RLCKs to the nucleus was reported earlier. For example, BIK1 localizes to the plasma membrane as well as to the nucleus (Lal et al., 2018). At the plasma membrane it interacts with immune receptors such as FLS2 and EFR, and in the nucleus BIK1 transphos- 
phorylates WRKY33, WRKY50 and WRKY57 transcription factors that negatively regulate SA and JA signaling (Lal et al., 2018). Possibly, CRK43 could fulfill similar functions in the nucleus.

The main function of the nucleolus is ribosomal RNA synthesis and ribosome biogenesis (Kalinina et al., 2018). Besides this, there is growing evidence that the nucleolus is also implicated in other functions such as regulation of cell cycle, growth and development, senescence, gene silencing as well as responses to biotic and abiotic stresses in plants (Kalinina et al., 2018). For example, it was shown that many proteins involved in diverse RNA silencing pathways accumulate in the nucleolar periphery in Arabidopsis (Pontes et al., 2013). Moreover, the potato and Nicotiana tabacum homologues of the Arabidopsis PUB17 E3 ubiquitin ligase, a positive regulator of plant disease resistance, localized especially to the nucleolus upon transient expression in $N$. benthamiana. It was demonstrated that PUB17 is involved in the early activation of PTI gene expression and silencing of NtPUB17 or NbPUB17 compromised R protein mediated but not PAMP induced cell death (He et al., 2015).

It is further known that a large number of viruses and also a number of other plant pathogens target the nucleolus to control the disease process (Kalinina et al., 2018). For example, several effectors of the oomycete pathogen Hyaloperonospora arabidopsidis have been shown to localize to the nucleolus of plant cells and regulate defense responses. One nucleolar $H$. arabidopsis effector interacts with the mediator subunit 19A, that is necessary for the association of RNA polymerase II with transcriptional regulators. This interaction leads to mediator degradation which switches transcription of plant defense genes from the SA to the JA/ ET pathway (Caillaud et al., 2013). Another nucleolar localized H. arabidopsidis effector was suggested to interact with a host protein involved in programmed cell death induction (Leonelli et al., 2011).

In humans, it was shown that proteins with a nucleolar detention signal are targeted by noncoding RNAs, which leads to immobilization in the nucleolus. Since these immobilized proteins can be released again upon demand, the nucleolus serves as a storage location for these proteins (Audas et al., 2014). Nevertheless, it is not known yet if a similar pathway is present in plants.

Since there are no published data available regarding localization of plant RLCKs in the nucleolus, it is not clear which function CRK43 might fulfill in this cellular compartment. 


\subsection{PAD4}

Mapping of a recessive cerk1-4 suppressor mutant line identified in the forward genetic screen revealed that all suppressor plants carried a single amino acid exchange of serine ${ }^{499}$ to phenylalanine in PHYTOALEXIN-DEFICIENT 4 (PAD4) (3.1.3). PAD4 is involved in SA signaling and the exaggerated cerkl-4 cell death phenotype is SA-dependent. Accordingly, cell death cannot be established in the cerk1-4 pad4-1 double mutant (Petutschnig et al., 2014). Since a functional PAD4 is known to be required for cerkl-4 cell death induction, it is most likely that the observed serine ${ }^{499}$ to phenylalanine exchange is the causative suppressor mutation in this line.

PAD4 is a lipase-like protein with a C-terminal lipase-like domain and an N-terminal EDS1PAD4 (EP)-domain that lacks homologies to other domains. Two PAD4 sequence-related proteins, ENHANCED DISEASE SUSCEPTIBILITY1 (EDS1) and SENESCENCE ASSOCIATED GENE 101 (SAG101) are present in Arabidopsis (Feys et al., 2001; Feys et al., 2005). EDS1 is able to form a complex with PAD4 to promote the accumulation of SA in basal resistance or with SAG101 to promote HR cell death in TIR-NLR mediated immunity (Feys et al., 2001; Feys et al., 2005; Cui et al., 2017 Gantner et al., 2019; Lapin et al., 2019). The EDS1-SAG101 complex was crystallized and both the N-terminal lipase-like domain and the C-terminal EP-domain are necessary for heterodimer formation (Wagner et al., 2013). Based on the structure of EDS1-SAG101, a very similar structure was modelled for EDS1PAD4 (Wagner et al., 2013). In both hetero-complexes a large interface for interaction with other proteins or ligands is formed between three long $\alpha$-helices resulting in a helical groove (Figure S9). A large cavity between the two EP domains creates a second C-terminal interface for protein-protein interaction or ligand binding (Wagner et al., 2013).

In general, an exchange of a polar neutral amino acid to a non-polar aromatic amino acid, like serine $^{499}$ to phenylalanine in the PAD4-14 mutant identified in the screen, could interfere with protein structure and stability. The PAD4-14 mutation is located in the EP domain and the mutated serine residue is conserved in SAG101, but not in EDS1 (Figure 42B). This conserved serine residue is located at the end of an $\alpha$-helix close to one of the long $\alpha$-helices forming the helical groove (Figure S9). This might result in alteration of one of the two interfaces, potentially making PAD4-14 unable to interact effectively with other proteins or ligands. Alternatively, it is also possible that the PAD4-14 protein is unstable because of an altered protein structure. Since there is a commercial PAD4 antibody available, protein stability could be tested via Western Blot analysis. 


\subsection{Potential roles of CRK7 and CRK43 in cerk1-4 cell death signaling}

Although several components required for cerkl-4 cell death have been identified yet, it is still unclear how these components interact with each other for cell death induction. Moreover, it is most likely that not all necessary components have been discovered so far. In this work, CRK7 and CRK43 were identified as novel components required for cerk1-4 cell death induction and possible functions of these CRKs will be discussed in the following.

Since CRK7 is an RLK, it is most likely that it binds a ligand with its ectodomain leading to signal transduction. CRKs have a number of highly conserved cysteine residues in their DUF26 domains and in general cysteine residues are sensitive to redox-modifications. Therefore it was speculated that CRKs could potentially act as ROS-receptors (Wrzaczek et al., 2010). However, recently the structure of the PDLP5 ectodomain containing two DUF26 domains was crystallized and shows strong similarity to the structure of fungal lectins (Vaattovaara et al., 2019). Since fungal lectins are carbohydrate-binding proteins (van Eerde et al., 2014; Zhang et al., 2017), it was suggested that DUF26 domain containing proteins might be involved in the recognition of carbohydrates as well (Vaattovaara et al., 2019). Indeed, mannose binding ability was demonstrated for the single DUF26 domain containing protein GNK2 from Ginkgo biloba (Miyakawa et al., 2014) and for the maize protein AFP1 that contains two DUF26 domains (Ma et al., 2018). Similarly, CRK7 could be involved in carbohydrate binding. The residues crucial for mannose binding in GNK2 (Miyakawa et al., 2014) are not conserved in CRK7 (Figure S1A), but it was suggested that CRK7 is involved in binding of a yet unidentified polysaccharide from Staphylococcus epidermidis leading to cell death induction (Dinischiotu, 2011).

One idea is that CRK7 could be involved in binding of glycosylated proteins or peptides. Five $\mathrm{N}$-glycosylation sites were determined in the CERK1 ectodomain (Liu et al., 2012) and it is tempting to speculate that CRK7 might bind glycosylated domains of either full length CERK1 (Figure 43 a) or the CERK1 ectodomain (Figure $43 \mathrm{~b}$ ) to monitor the integrity of this important immune receptor. The amino acid exchange in CERK1-4 (Petutschnig et al., 2014) is located directly next to an asparagine that is glycosylated (Liu et al., 2012). Therefore it might be possible that CERK1-4 glycosylation is altered and CRK7 binding of the differentially glycosylated domains might be abolished, leading to cell death induction. Alternatively it would be also possible the other way around, that CRK7 recognizes altered glycosylation of the CERK1-4 protein (Figure $43 \mathrm{c}$ ) or glycopeptides in turnover products of the CERK1-4 ectodomain (Figure $43 \mathrm{~d}$ ) as DAMP molecules, resulting in cell death formation. 


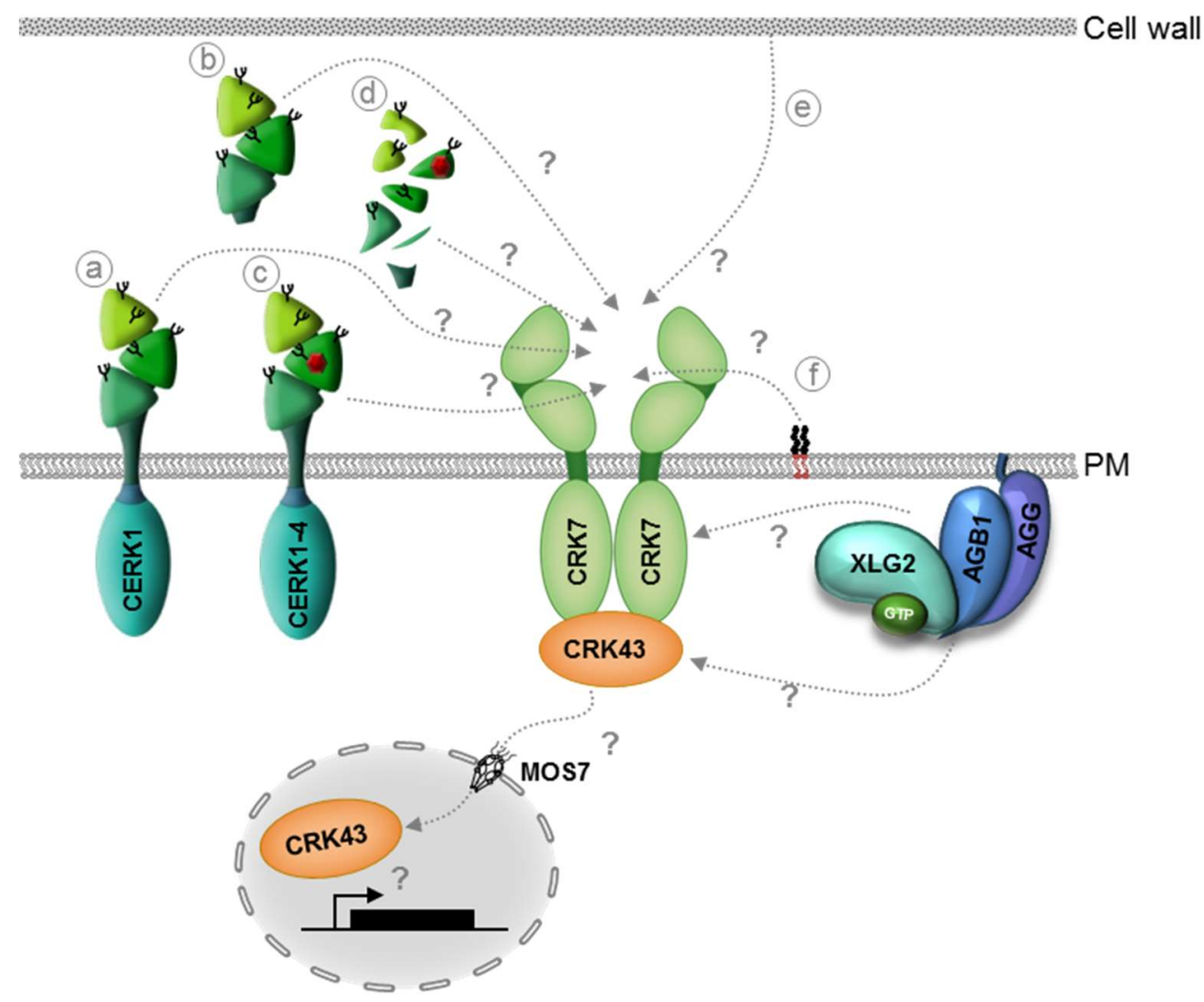

\section{Figure 43: Potential roles of CRK7 and CRK43 in cerk1-4 cell death induction.}

Potential molecules recognized by CRK7 are full length CERK1 (a) or CERK1-4 (c), the CERK1 ectodomain (b), turnover products of the CERK1-4 ectodomain (d), cell wall derived molecules (e) or glycolipids (f). Glycosylation (black) of CERK1 or its derivatives may be important for recognition. CRK43 might interact with CRK7 and is released from the complex potentially upon ligand perception. Subsequently, it might be translocated to the nucleus where it could potentially lead to altered gene expression. Import of CRK43 into the nucleus might require the nucleoporin MOS7/ NUP88. The heterotrimeric Gprotein complex AGG, AGB1 and XLG2 might interact with CRK7 and/ or CRK43 either direct or indirectly in a complex. $\mathrm{PM}=$ plasma membrane.

The hypothesis that recognition of CERK1 or CERK1-4 glycoproteins might be crucial for regulation of the exaggerated cerk1-4 dependent cell death phenotype is supported by preliminary data that suggest that the sugar transporter UDP-GLCNAC TRANSPORTER 1 (UGNT1) is necessary for cell death induction (A. Vasquez, unpublished). UGNT1 is required to transport uridine diphosphate $\mathrm{N}$-acetylglucosamine (UDP-GlcNAc) from the cytosol into the Golgi apparatus, where it is needed for N-glycan maturation (Ebert et al., 2018). Nevertheless, it might be also possible that CRK7 recognizes CERK1, the CERK1 ectodomain, CERK1-4 or turnover products of the CERK1-4 ectodomain directly and independent of glycosylation.

At the current state, it cannot be excluded that CRK7 might act more downstream in cerk1-4 cell death induction and is not involved in binding any ligands that directly stem from the CERK1/ CERK1-4 protein. 
Given the proposed lectin function of DUF26 domains (Vaattovaara et al., 2019), other potential CRK7 ligands are plant cell wall components (Figure 43 e) or glycolipids (Figure 43 f). In general, a potential CRK7 ligand should be present at a low level in WT plants and should accumulate upon infection with powdery mildews and in senescent plants. Nevertheless, it is not clear yet if a ligand is necessary for CRK7 dimerization, since CRK7 was able to dimerize upon transient expression in $N$. benthamiana. However, this potentially ligandfree dimerization could be also an effect of CRK7 overexpression.

The other CRK identified as crucial for cerk1-4 dependent cell death is CRK43, which lacks the ecto- and transmembrane domain and is therefore considered a CRCK (Bourdais et al., 2015; Vaattovaara et al., 2019). It is a common theme that receptor-like cytoplasmic kinases associate with membrane-localized receptors to form complexes and RLCKs are often released from these complexes for signal transduction upon ligand perception (Liang and Zhou, 2018). Since another Arabidopsis CRCK, CRK45, was shown to interact with the full length CRK CRK36 (Tanaka et al., 2012), it is an attractive hypothesis that CRK43 might interact with CRK7 to control cerk1-4 cell death formation (Figure 43).

CRK43-mCitrine localized predominantly to the cell periphery but also to the nucleus in $\mathrm{Ar}$ abidopsis. In the future it will be investigated, if both cell periphery and nuclear localization are necessary for CRK43 function in cell death induction. It is conceivable that CRK43 might interact with $\mathrm{CRK} 7$, be released from the complex upon ligand perception and might be translocated to the nucleus for signal transduction. CRK43-mCitrine localized predominantly to the nucleus/ nucleolus upon expression in $N$. benthamiana, but mainly to the cell periphery in Arabidopsis. These observations support the hypothesis that the potential interaction with CRK7 keeps CRK43 at the cell periphery. In N. benthamiana CRK7 is not present and $N$. benthamiana orthologues may not efficiently interact with CRK43, potentially leading to translocation of CRK43 to the nucleus. Upon CRK43 overexpression, a higher amount of CRK43 proteins compared to CRK7 would be present in the cell, possibly leading to translocation of cytoplasmic CRK43 to the nucleus, resulting in cell death formation.

A potential candidate for nuclear import of CRK43 is MOS7, a nucleoporin that was shown to be necessary for cerk1-4 cell death induction (Genenncher et al., 2017).

Amino acids crucial for kinase activity (Hanks and Hunter, 1995) are conserved in CRK43. Therefore it is conceivable that CRK43 might phosphorylate transcription factors inside the nucleus to regulate defense gene expression (Figure 43).

Besides CRK7 and CRK43, also a heterotrimeric G-protein complex consisting of AGG, AGB1 and XLG2 functions in cerk1-4 cell death signaling (E. Petutschnig, M. Stolze, C. 
Meusel, unpublished). Potentially, these G-proteins might interact with CRK7 and/ or CRK43 (Figure 43). Since there is no experimental evidence yet that these proteins directly interact, it might also be possible that interaction is bridged by another yet unidentified complex partner. Taken together, many questions are still open and future work will hopefully help to understand the mechanism underlying cerkl-4 cell death induction.

\section{$\underline{4.5 \text { Outlook }}$}

In this work it was shown that CRK7 and CRK43 are necessary for cerk1-4 dependent cell death formation. Additionally it is known that the extra-large G-protein XLG2 and its complex partners AGB1 and AGG1/2 (M. Stolze, C. Meusel, J. Anders, E. Petutschnig, unpublished), the nucleoporine MOS7 (Genennecher et al., 2017) as well as SA synthesis and signaling components (Petutschnig et al., 2014) are required for exaggerated cell death in cerk1-4. How these proteins interact with each other and how the mechanism works in detail remains to be determined.

One aspect future work should focus on is the identification of the CRK7 ligand. It was shown that infiltration with killed Staphylococcus epidermidis triggers CRK7-dependent cell death in Arabidopsis and biochemical assays suggest that the responsible substance is a polysaccharide (Dinischiotu, 2011). If cell death cannot be induced by infiltration of autoclaved $S$. epidermidis into crk7-4 mutant plants, the CRK7 ligand could be isolated from S. epidermidis. This might not only help to understand the role of CRK7 in cerk1-4 cell death induction, but could potentially also provide general concepts about the function of CRKs. Since CRKs occur in families of closely related genes, it should be tested, if the closest CRK7 homologues CRK6 and CRK8 are also involved in cerk1-4 cell death induction.

In this work it was shown that CRK7 homodimerizes and/or oligomerizes whereas CRK7-4 is unable to form homodimers (3.1.6.2). To further confirm these findings, FLIM-FRET (Fluorescence Lifetime Imaging Microscopy-Förster Resonance Energy Transfer) experiments should be conducted with CRK7-mScarlet-I and CRK7-mCitrine and CRK7-4 fused to the same fluorescence proteins.

Moreover, Co-IP experiments should be performed to investigate a possible direct interaction between CRK7/ CRK7-4 and the ectodomain of CERK1/ CERK1-4.

Concerning CRK43, further experiments should be conducted to investigate if CRK43 cell periphery, nuclear and/ or nucleolar localization are required for the cell death phenotype. To test this, the predicted nuclear and nucleolar localization signals could be mutated and locali- 
zation changes should be confirmed in $N$. benthamiana. Constructs with clear localization changes should be transformed into cerk1-4 crk43-2 plants to investigate if cell death is induced. If these experiments reveal that the nuclear localization of CRK43 is crucial for cell death formation, it could be examined if MOS7 is necessary for the nuclear import of CRK43 by localization studies in the $\operatorname{mos} 7$ mutant. To investigate if the potential interaction of CRK7 and CRK43 is crucial for CRK43 cell periphery localization, confocal microscopy could be performed with CRK43-mCitrine in the crk7 mutant background.

In general, CRK43-mCitrine expression under the native promotor was very weak, making microscopy challenging, but overexpression of CRK43-mCitrine appears to be lethal. Therefore it would make sense to clone CRK43 under the control of an inducible promotor for further microscopic analyses.

Amino acids crucial for kinase activity are conserved in CRK43. Nevertheless enzymatic activity of CRK43 should be confirmed experimentally.

In summary, these experiments should help to understand the roles of CRK7 and CRK43 in the cerk1-4 cell death reaction. 


\section{References}

Acharya, B. R.; Raina, S.; Maqbool, S. B.; Jagadeeswaran, G.; Mosher, S. L.; Appel, Heidi M. (2007): Overexpression of CRK13, an Arabidopsis cysteine-rich receptor-like kinase, results in enhanced resistance to Pseudomonas syringae. In: The Plant journal for cell and molecular biology 50 (3), p. 488-499.

Alfano, J. R.; Collmer, A. (1997): The type III (Hrp) secretion pathway of plant pathogenic bacteria: trafficking harpins, Avr proteins, and death. In: Journal of bacteriology 179 (18), p. 56555662.

Almsherqi, Z. A.; Landh, T.; Kohlwein, S. D.; Deng, Y. (2009): Chapter 6 Cubic Membranes. In: International review of cell and molecular biology 274, p. 275-342.

Alonso, J. M.; Stepanova, A. N.; Leisse, T. J.; Kim, C. J.; Chen, H.; Shinn, P. et al. (2003): Genomewide insertional mutagenesis of Arabidopsis thaliana. In: Science (New York, N.Y.) 301 (5633), p. 653- 657.

Amari, K.; Boutant, E.; Hofmann, C.; Schmitt-Keichinger, C.; Fernandez-Calvino, L.; Didier, P. et al. (2010): A family of plasmodesmal proteins with receptor-like properties for plant viral movement proteins. In: PLoS pathogens 6 (9), e1001119.

Amor, B. B.; Shaw, S. L.; Oldroyd, G. E. D.; Maillet, F.; Penmetsa, R. V.; Cook, D. et al. (2003): The NFP locus of Medicago truncatula controls an early step of Nod factor signal transduction upstream of a rapid calcium flux and root hair deformation. In: The Plant journal: for cell and molecular biology 34 (4), p. 495-506.

Antolín-Llovera, M.; Ried, M. K.; Binder, A.; Parniske, M. (2012): Receptor kinase signaling pathways in plant-microbe interactions. In: Annual review of phytopathology 50, p. 451-473.

Antolín-Llovera, M.; Ried, M. K.; Parniske, M. (2014): Cleavage of the SYMBIOSIS RECEPTORLIKE KINASE ectodomain promotes complex formation with Nod factor receptor 5. In: Current biology: CB 24 (4), p. 422-427.

Ao, Y.; Li, Z.; Feng, D.; Xiong, F.; Liu, J.; Li, J.; Wang, M.; Liu, B; Wang, H. (2014): OsCERK1 and OsRLCK176 play important roles in peptidoglycan and chitin signaling in rice innate immunity. In: The Plant journal: for cell and molecular biology 80 (6), p. 1072-1084.

Arribas, J.; Borroto, A. (2002): Protein ectodomain shedding. In: Chemical reviews 102 (12), p. $4627-4638$.

Arrighi, J.-F.; Barre, A.; Amor, B. B.; Bersoult, A.; Soriano, L. C.; Mirabella, R. et al. (2006): The Medicago truncatula lysin corrected motif-receptor-like kinase gene family includes NFP and new nodule-expressed genes. In: Plant physiology 142 (1), p. 265-279.

Audas, T. E.; Jacob, M. D.; Lee, S. (2012): The nucleolar detention pathway: A cellular strategy for regulating molecular networks. In: Cell cycle (Georgetown, Tex.) 11 (11), p. 2059-2062.

Ausubel, F. M. (2005): Are innate immune signaling pathways in plants and animals conserved? In: Nature immunology 6 (10), p. 973-979.

Backer, R.; Naidoo, S.; van den Berg, N. (2019): The NONEXPRESSOR OF PATHOGENESIS-RELATED GENES 1 (NPR1) and Related Family: Mechanistic Insights in Plant Disease Resistance. In: Frontiers in Plant Science 10, p. 102.

Bartels, S.; Boller, T. (2015): Quo vadis, Pep? Plant elicitor peptides at the crossroads of immunity, stress, and development. In: Journal of experimental botany 66 (17), p. 5183-5193.

Bartels, S.; Lori, M.; Mbengue, M.; van Verk, M.; Klauser, D.; Hander, T. et al. (2013): The family of Peps and their precursors in Arabidopsis: differential expression and localization but similar induction of pattern-triggered immune responses. In: Journal of experimental botany 64 (17), p. 5309-5321. 
Bateman, A.; Bycroft, M. (2000): The structure of a LysM domain from E. coli membrane-bound lytic murein transglycosylase D (MltD). In: Journal of molecular biology 299 (4), p. 11131119.

Birnboim, H. C.; Doly, J. (1979): A rapid alkaline extraction procedure for screening recombinant plasmid DNA. In: Nucleic acids research 7 (6), p. 1513-1523.

Boller, T.; Felix, G. (2009): A renaissance of elicitors: perception of microbe-associated molecular patterns and danger signals by pattern-recognition receptors. In: Annual review of plant biology 60, p. 379-406.

Bologna, G.; Yvon, C.; Duvaud, S.; Veuthey, A.-L. (2004): N-Terminal myristoylation predictions by ensembles of neural networks. In: Proteomics 4 (6), p. 1626-1632.

Bolton, M. D.; van Esse, H. P.; Vossen, J. H.; de Jonge, R.; Stergiopoulos, I.; Stulemeijer, I. J. E. et al. (2008): The novel Cladosporium fulvum lysin motif effector Ecp6 is a virulence factor with orthologues in other fungal species. In: Molecular microbiology 69 (1), p. 119-136.

Borràs, E.; Sabidó, E. (2017): What is targeted proteomics? A concise revision of targeted acquisition and targeted data analysis in mass spectrometry. In: Proteomics 17 (17-18).

Bourdais, G.; Burdiak, P.; Gauthier, A.; Nitsch, L.; Salojärvi, J.; Rayapuram, C. et al. (2015): LargeScale Phenomics Identifies Primary and Fine-Tuning Roles for CRKs in Responses Related to Oxidative Stress. In: PLoS genetics 11 (7), e1005373.

Boyes, D. C.; Zayed, A. M.; Ascenzi, R.; McCaskill, A. J.; Hoffman, N. E.; Davis, K. R.; Görlach, J. (2001): Growth stage-based phenotypic analysis of Arabidopsis: a model for high throughput functional genomics in plants. In: The Plant cell 13 (7), p. 1499-1510.

Bradford, M. M. (1976): A rapid and sensitive method for the quantitation of microgram quantities of protein utilizing the principle of protein-dye binding. In: Analytical biochemistry 72, p. $248-254$.

Broghammer, A.; Krusell, L.; Blaise, M.; Sauer, J.; Sullivan, J. T.; Maolanon, N. et al. (2012): Legume receptors perceive the rhizobial lipochitin oligosaccharide signal molecules by direct binding. In: Proceedings of the National Academy of Sciences of the United States of America 109 (34), p. 13859-13864.

Brunkard, J. O.; Zambryski, P. C. (2017): Plasmodesmata enable multicellularity: new insights into their evolution, biogenesis, and functions in development and immunity. In: Current opinion in plant biology 35, p. 76-83.

Buist, G.; Steen, A.; Kok, J.; Kuipers, O. P. (2008): LysM, a widely distributed protein motif for binding to (peptido)glycans. In: Molecular microbiology 68 (4), p. 838-847.

Burch-Smith, T. M.; Stonebloom, S.; Xu, M.; Zambryski, P. C. (2011): Plasmodesmata during development: re-examination of the importance of primary, secondary, and branched plasmodesmata structure versus function. In: Protoplasma 248 (1), p. 61-74.

Butaye, K. M. J.; Goderis, I. J. W. M.; Wouters, P. F. J.; Pues, J. M.-T. G.; Delauré, S. L.; Broekaert, W. F. et al. (2004): Stable high-level transgene expression in Arabidopsis thaliana using gene silencing mutants and matrix attachment regions. In: The Plant journal: for cell and molecular biology 39 (3), p. 440-449.

Büttner, D. (2016): Behind the lines-actions of bacterial type III effector proteins in plant cells. In: FEMS microbiology reviews 40 (6), p. 894-937.

Caillaud, M.-C.; Asai, S.; Rallapalli, G.; Piquerez, S.; Fabro, G.; Jones, J. D. G. (2013): A downy mildew effector attenuates salicylic acid-triggered immunity in Arabidopsis by interacting with the host mediator complex. In: PLoS biology 11 (12), e1001732. 
Caillaud, M.-C.; Wirthmueller, L.; Sklenar, J.; Findlay, K.; Piquerez, S. J. M.; Jones, A. M. E. et al. (2014): The plasmodesmal protein PDLP1 localises to haustoria-associated membranes during downy mildew infection and regulates callose deposition. In: PLoS pathogens 10 (10), e1004496.

Cao, H.; Li, X.; Dong, X. (1998): Generation of broad-spectrum disease resistance by overexpression of an essential regulatory gene in systemic acquired resistance. In: Proceedings of the National Academy of Sciences of the United States of America 95 (11), p. 6531-6536.

Cao, Y.; Liang, Y.; Tanaka, K.; Nguyen, C. T.; Jedrzejczak, R. P.; Joachimiak, A.; Stacey, G. (2014): The kinase LYK5 is a major chitin receptor in Arabidopsis and forms a chitin-induced complex with related kinase CERK1. In: eLife 3.

Chakravorty, D.; Gookin, T. E.; Milner, M. J.; Yu, Y.; Assmann, S. M. (2015): Extra-Large G Proteins Expand the Repertoire of Subunits in Arabidopsis Heterotrimeric G Protein Signaling. In: Plant physiology 169 (1), p. 512-529.

Chandran, D.; Inada, N.; Hather, G.; Kleindt, C. K.; Wildermuth, M. C. (2010): Laser microdissection of Arabidopsis cells at the powdery mildew infection site reveals site-specific processes and regulators. In: Proceedings of the National Academy of Sciences of the United States of America 107 (1), p. 460-465.

Chen, K.; Du, L.; Chen, Z. (2003): Sensitization of defense responses and activation of programmed cell death by a pathogen-induced receptor-like protein kinase in Arabidopsis. In: Plant molecularbiology 53 (1-2), p. 61-74.

Chen, K.; Fan, B.; Du, L.; Chen, Z. (2004): Activation of hypersensitive cell death by pathogen-induced receptor-like protein kinases from Arabidopsis. In: Plant molecular biology 56 (2), p. 271-283.

Chen, Z. (2001): A superfamily of proteins with novel cysteine-rich repeats. In: Plant physiology 126 (2), p. 473-476.

Chen, Z.; Agnew, J. L.; Cohen, J. D.; He, P.; Shan, L.; Sheen, J.; Kunkel, B. N. (2007): Pseudomonas syringae type III effector AvrRpt2 alters Arabidopsis thaliana auxin physiology. In: Proceedings of the National Academy of Sciences of the United States of America 104 (50), p. 20131-20136.

Cheng, C.-Y.; Krishnakumar, V.; Chan, A. P.; Thibaud-Nissen, F.; Schobel, S.; Town, C. D. (2017): Araport11: a complete reannotation of the Arabidopsis thaliana reference genome. In: The Plant journal: for cell and molecular biology 89 (4), p. 789-804.

Chern, M.; Xu, Q.; Bart, R. S.; Bai, W.; Ruan, D.; Sze-To, W. H. et al. (2016): A Genetic Screen Identifies a Requirement for Cysteine-Rich-Receptor-Like Kinases in Rice NH1 (OsNPR1)Mediated Immunity. In: PLoS genetics 12 (5), e1006049.

Cheval, Cecilia; Faulkner, Christine (2018): Plasmodesmal regulation during plant-pathogen interactions. In: The New phytologist 217 (1), S. 62-67.

Cheval, C.; Samwald, S.; Johnston, M. G.; de Keijzer, J.; Breakspear, A.; Liu, X. et al. (2020): Chitin perception in plasmodesmata characterizes submembrane immune-signaling specificity in plants. In: Proceedings of the National Academy of Sciences of the United States of America 117 (17), p. 9621-9629.

Chinchilla, D.; Bauer, Z.; Regenass, M.; Boller, T.; Felix, G. (2006): The Arabidopsis receptor kinase FLS2 binds flg22 and determines the specificity of flagellin perception. In: The Plant cell 18 (2), p. $465-476$.

Chinchilla, D.; Zipfel, C.; Robatzek, S.; Kemmerling, B.; Nürnberger, T.; Jones, J. D. G. et al. (2007): A flagellin-induced complex of the receptor FLS2 and BAK1 initiates plant defence. In: Nature 448 (7152), p. 497-500. 
Choi, H. W.; Klessig, D. F. (2016): DAMPs, MAMPs, and NAMPs in plant innate immunity. In: BMC plant biology 16 (1), p. 232.

Clough, S. J.; Bent, A. F. (1998): Floral dip: a simplified method for Agrobacterium-mediated transformation of Arabidopsis thaliana. In: The Plant journal: for cell and molecular biology $16(6)$, p. 735-743.

Copenhaver, G. P.; Nickel, K.; Kuromori, T.; Benito, M. I.; Kaul, S.; Lin, X. et al. (1999): Genetic definition and sequence analysis of Arabidopsis centromeres. In: Science (New York, N.Y.) 286 (5449), p. 2468-2474.

Cranfill, P. J.; Sell, B. R.; Baird, M. A.; Allen, J. R.; Lavagnino, Z.; de Gruiter, H. M. et al. (2016): Quantitative assessment of fluorescent proteins. In: Nature methods 13 (7), p. 557-562.

Cui, H.; Gobbato, E.; Kracher, B.; Qiu, J.; Bautor, J.; Parker, J. E. (2017): A core function of EDS1 with PAD4 is to protect the salicylic acid defense sector in Arabidopsis immunity. In: The New phytologist 213 (4), p. 1802-1817.

Cui, H.; Tsuda, K.; Parker, J. E. (2015): Effector-triggered immunity: from pathogen perception to robust defense. In: Annual review of plant biology 66, p. 487-511.

Cui, H.; Wang, Y.; Xue, L.; Chu, J.; Yan, C.; Fu, J. et al. (2010): Pseudomonas syringae effector protein AvrB perturbs Arabidopsis hormone signaling by activating MAP kinase 4. In: Cell host \& microbe 7 (2), p. 164-175.

Czernic, P.; Visser, B.; Sun, W.; Savouré, A.; Deslandes, L.; Marco, Y. et al. (1999): Characterization of an Arabidopsis thaliana receptor-like protein kinase gene activated by oxidative stress and pathogen attack. In: The Plant journal: for cell and molecular biology 18 (3), p. 321-327.

Dangl, J. L.; Jones, J. D. G. (2019): A pentangular plant inflammasome. In: Science (New York, N.Y.) 364(6435), p. 31-32.

Dangl, J. L.; Horvath, D. M.; Staskawicz, B. J. (2013): Pivoting the plant immune system from dissection to deployment. In: Science (New York, N.Y.) 341 (6147), p. 746-751.

Ding, L.; Pandey, S.; Assmann, S. M. (2008): Arabidopsis extra-large G proteins (XLGs) regulate root morphogenesis. In: The Plant journal: for cell and molecular biology 53 (2), p. 248-263.

Dinischiotu, N. (2011): STER1, a novel receptor-like kinase, functions in MAMP signaling in Arabidopsis.

de Buck, S.; de Paepe, A.; Depicker, A. (2013): Transgene Expression plant transgene expression in Plants plant, Control of. In: Paul Christou, Roxana Savin, Barry A. Costa-Pierce, Ignacy Misztal und C. Bruce A. Whitelaw (Hg.): Sustainable Food Production. New York, NY: Springer, p. 1570-1593.

de Jonge, R.; Thomma, B. P. H. J. (2009): Fungal LysM effectors: extinguishers of host immunity? In: Trends in microbiology 17 (4), p. 151-157.

de Mita, S.; Streng, A.; Bisseling, T.; Geurts, R. (2014): Evolution of a symbiotic receptor through gene duplications in the legume-rhizobium mutualism. In: The New phytologist 201 (3), p. 961-972.

de Oliveira, M. V. V.; Xu, G.; Li, B.; Souza V., de Luciano; Meng, X.; Chen, X. et al. (2016): Specific control of Arabidopsis BAK1/SERK4-regulated cell death by protein glycosylation. In: Nature plants 2, p. 15218.

de Wit, P. J. G. M. (2007): How plants recognize pathogens and defend themselves. In: Cellular and molecular life sciences: CMLS 64 (21), p. 2726-2732.

de Wit, P. J. G. M.; Mehrabi, R.; van den Burg, H. A.; Stergiopoulos, I. (2009): Fungal effector proteins: past, present and future. In: Molecular plant pathology 10 (6), p. 735-747. 
Desaki, Y.; Kouzai, Y.; Ninomiya, Y.; Iwase, R.; Shimizu, Y.; Seko, K. et al. (2018): OsCERK1 plays a crucial role in the lipopolysaccharide-induced immune response of rice. In: The New phytologist 217 (3), S. 1042-1049.

Dodds, P. N.; Lawrence, G. J.; Catanzariti, A.-M.; Teh, T.; Wang, C.-I. A.; Ayliffe, M. A. et al. (2006): Direct protein interaction underlies gene-for-gene specificity and coevolution of the flax resistance genes and flax rust avirulence genes. In: Proceedings of the National Academy of Sciences of the United States of America 103 (23), p. 8888-8893.

Dodds, P. N.; Rathjen, J. P. (2010): Plant immunity: towards an integrated view of plant-pathogen interactions. In: Nature reviews. Genetics 11 (8), p. 539-548.

Domazakis, E.; Wouters, D.; Visser, R. G. F.; Kamoun, S.; Joosten, M. H. A. J.; Vleeshouwers, V. G. A. A. (2018): The ELR-SOBIR1 Complex Functions as a Two-Component Receptor-Like Kinase to Mount Defense Against Phytophthora infestans. In: Molecular plant-microbe interactions: MPMI 31 (8), p. 795-802.

Du, D.; Liu, M.; Xing, Y.; Chen, X.; Zhang, Y.; Zhu, M. et al. (2019): Semi-dominant mutation in the cysteine-rich receptor-like kinase gene, ALS1, conducts constitutive defence response in rice. In: Plant biology (Stuttgart, Germany) 21 (1), p. 25-34.

Du, L.; Chen, Z. (2000): Identification of genes encoding receptor-like protein kinases as possible targets of pathogen- and salicylic acid-induced WRKY DNA-binding proteins in Arabidopsis. In: The Plant journal: for cell and molecular biology 24 (6), p. 837-847.

Durrant, W. E.; Dong, X. (2004): Systemic acquired resistance. In: Annual review of phytopathology 42, p. 185-209.

Ebert, B.; Rautengarten, C.; McFarlane, H. E.; Rupasinghe, T.; Zeng, W.; Ford, K. et al. (2018): A Golgi UDP-GlcNAc transporter delivers substrates for N-linked glycans and sphingolipids. In: Nature plants 4 (10), p. 792-801.

El-Gebali, S.; Mistry, J.; Bateman, A.; Eddy, S. R.; Luciani, A.; Potter, S. C. et al. (2019): The Pfam protein families database in 2019. In: Nucleic acids research 47 (D1), D427-D432.

Erwig, J. (2016): Analysis of the subcellular behavior of Arabidopsis thaliana LysM-proteins and their role in plant immunity.

Erwig, J.; Ghareeb, H.; Kopischke, M.; Hacke, R.; Matei, A.; Petutschnig, E. K.; Lipka, V. (2017): Chitin-induced and CHITIN ELICITOR RECEPTOR KINASE1 (CERK1) phosphorylationdependent endocytosis of Arabidopsis thaliana LYSIN MOTIF-CONTAINING RECEPTORLIKE KINASE5 (LYK5). In: The New phytologist 215 (1), p. 382-396.

Faulkner, C.; Petutschnig, E. K.; Benitez-Alfonso, Y.; Beck, M.; Robatzek, S.; Lipka, V.; Maule, A. J. (2013): LYM2-dependent chitin perception limits molecular flux via plasmodesmata. In: Proceedings of the National Academy of Sciences of the United States of America 110 (22), p. 9166-9170.

Fellbrich, G.; Romanski, A.; Varet, A.; Blume, B.; Brunner, F.; Engelhardt, S. et al. (2002): NPP1, a Phytophthora-associated trigger of plant defense in parsley and Arabidopsis. In: The Plant journal: for cell and molecular biology 32 (3), p. 375-390.

Ferrero, S.; Grados-Torrez, R. E.; Leivar, P.; Antolín-Llovera, M.; López-Iglesias, C.; Cortadellas, N. et al. (2015): Proliferation and Morphogenesis of the Endoplasmic Reticulum Driven by the Membrane Domain of 3-Hydroxy-3-Methylglutaryl Coenzyme A Reductase in Plant Cells. In: Plant physiology 168 (3), p. 899-914.

Feys, B. J.; Moisan, L. J.; Newman, M. A.; Parker, J. E. (2001): Direct interaction between the Arabidopsis disease resistance signaling proteins, EDS1 and PAD4. In: The EMBO journal 20(19), p. 5400-5411. 
Feys, B. J.; Wiermer, M.; Bhat, R. A.; Moisan, L. J.; Medina-Escobar, N.; Neu, C. et al. (2005): Arabidopsis SENESCENCE-ASSOCIATED GENE101 stabilizes and signals within an ENHANCED DISEASE SUSCEPTIBILITY1 complex in plant innate immunity. In: The Plant cell 17 (9), p. 2601-2613.

Fliegmann, J.; Canova, S.; Lachaud, C.; Uhlenbroich, S.; Gasciolli, V.; Pichereaux, C. et al. (2013): Lipo-chitooligosaccharidic symbiotic signals are recognized by LysM receptor-like kinase LYR3 in the legume Medicago truncatula. In: ACS chemical biology 8 (9), p. 1900-1906.

Fliegmann, J.; Jauneau, A.; Pichereaux, C.; Rosenberg, C.; Gasciolli, V.; Timmers, A. C. J. et al. (2016): LYR3, a high-affinity LCO-binding protein of Medicago truncatula, interacts with LYK3, a key symbiotic receptor. In: FEBS letters 590 (10), p. 1477-1487.

Fritz-Laylin, L. K.; Krishnamurthy, N.; Tör, M.; Sjölander, K. V.; Jones, J. D. G. (2005): Phylogenomic analysis of the receptor-like proteins of rice and Arabidopsis. In: Plant physiology 138 (2), p. 611-623.

Fu, Z. Q.; Dong, X. (2013): Systemic acquired resistance: turning local infection into global defense. In: Annual review of plant biology 64, p. 839-863.

Gantner, J.; Ordon, J.; Kretschmer, C.; Guerois, R.; Stuttmann, J. (2019): An EDS1-SAG101 Complex Is Essential for TNL-Mediated Immunity in Nicotiana benthamiana. In: The Plant cell 31 (10), p. 2456-2474.

Gao, M.; Wang, X.; Wang, D.; Xu, F.; Ding, X.; Zhang, Z. et al. (2009): Regulation of cell death and innate immunity by two receptor-like kinases in Arabidopsis. In: Cell host \& microbe 6 (1), p. $34-44$.

Gao, X.; Ruan, X.; Sun, Y.; Wang, X.; Feng, B. (2018): BAKing up to Survive a Battle: Functional Dynamics of BAK1 in Plant Programmed Cell Death. In: Frontiers in plant science 9, p. 1913.

Gay, Nicholas J.; Gangloff, Monique (2007): Structure and function of Toll receptors and their ligands. In: Annual review of biochemistry 76, p. 141-165.

Genenncher, B.; Lipka, V.; Petutschnig, E. K.; Wiermer, M. (2017): Nucleoporin NUP88/MOS7 is required for manifestation of phenotypes associated with the Arabidopsis CHITIN ELICITOR RECEPTOR KINASE1 mutant cerk1-4. In: Plant signaling \& behavior 12 (5), e1313378.

Gimenez-Ibanez, S.; Hann, D. R.; Ntoukakis, V.; Petutschnig, E. K.; Lipka, V.; Rathjen, J. P. (2009a): AvrPtoB targets the LysM receptor kinase CERK1 to promote bacterial virulence on plants. In: Current biology: CB 19 (5), p. 423-429.

Gimenez-Ibanez, S.; Ntoukakis, V.; Rathjen, J. P. (2009b): The LysM receptor kinase CERK1 mediates bacterial perception in Arabidopsis. In: Plant signaling \& behavior 4 (6), p. 539-541.

Góliebran-Gómez, L.; Boller, T. (2002): Flagellin perception: a paradigm for innate immunity. In: Trends in plant science 7 (6), p. 251-256.

Gong, B.-Q.; Xue, J.; Zhang, N.; Xu, L.; Yao, X.; Yang, Q.-J. et al. (2017): Rice Chitin Receptor OsCEBiP Is Not a Transmembrane Protein but Targets the Plasma Membrane via a GPI Anchor. In: Molecular plant 10 (5), p. 767-770.

Gubaeva, E.; Gubaev, A.; Melcher, R. L. J.; Cord-Landwehr, S.; Singh, R.; El Gueddari, N. E.; Moerschbacher, B. M. (2018): 'Slipped Sandwich' Model for Chitin and Chitosan Perception in Arabidopsis. In: Molecular plant-microbe interactions : MPMI 31 (11), S. 1145-1153.

Gupta, R.; Jung, E.; Brunak, S. (2004): Prediction of N-glycosylation sites in human proteins. In: in preparation.

Gust, A. A. (2015): Peptidoglycan Perception in Plants. In: PLoS pathogens 11 (12), e1005275.

Gust, A. A.; Felix, G. (2014): Receptor like proteins associate with SOBIR1-type of adaptors to form bimolecular receptor kinases. In: Current opinion in plant biology 21, p. 104-111. 
Gust, A. A.; Willmann, R.; Desaki, Y.; Grabherr, H. M.; Nürnberger, T. (2012): Plant LysM proteins: modules mediating symbiosis and immunity. In: Trends in plant science 17 (8), p. 495-502.

Haapalainen, M.; Dauphin, A.; Li, C-M; Bailly, G.; Tran, D.; Briand, J. et al. (2012): HrpZ harpins from different Pseudomonas syringae pathovars differ in molecular interactions and in induction of anion channel responses in Arabidopsis thaliana suspension cells. In: Plant physiology and biochemistry: PPB 51, p. 168-174.

Haeussler, M.; Schönig, K.; Eckert, H.; Eschstruth, A.; Mianné, J.; Renaud, J.-B. et al. (2016): Evaluation of off-target and on-target scoring algorithms and integration into the guide RNA selection tool CRISPOR. In: Genome biology 17 (1), p. 148.

Halter, T.; Imkampe, J.; Blaum, B. S.; Stehle, T.; Kemmerling, B. (2014b): BIR2 affects complex formation of BAK1 with ligand binding receptors in plant defense. In: Plant signaling \& behavior 9.

Halter, T.; Imkampe, J.; Mazzotta, S.; Wierzba, M.; Postel, S.; Bücherl, C. et al. (2014a): The leucine-rich repeat receptor kinase BIR2 is a negative regulator of BAK1 in plant immunity. In: Current biology: $C B 24$ (2), p. 134-143.

Hanks, S. K.; Hunter, T. (1995): Protein kinases 6. The eukaryotic protein kinase superfamily: kinase (catalytic) domain structure and classification. In: FASEB journal: official publication of the Federation of American Societies for Experimental Biology 9 (8), p. 576-596.

Hanks, S. K.; Quinn, A. M.; Hunter, T. (1988): The protein kinase family: conserved features and deduced phylogeny of the catalytic domains. In: Science (New York, N.Y.) 241 (4861), p. 4252.

Hann, D. R.; Rathjen, J. P. (2007): Early events in the pathogenicity of Pseudomonas syringae on Nicotiana benthamiana. In: The Plant journal: for cell and molecular biology 49 (4), p. 607 618.

Hartwig, B.; James, G. V.; Konrad, K.; Schneeberger, K.; Turck, F. (2012): Fast isogenic mappingby-sequencing of ethyl methanesulfonate-induced mutant bulks. In: Plant physiology 160 (2), p. 591-600.

Hayafune, M.; Berisio, R.; Marchetti, R.; Silipo, A.; Kayama, M.; Desaki, Y. et al. (2014): Chitininduced activation of immune signaling by the rice receptor CEBiP relies on a unique sandwich-type dimerization. In: Proceedings of the National Academy of Sciences of the United States of America 111 (3), E404-13.

Hayashida, K.; Bartlett, A. H.; Chen, Y.; Park, P. W. (2010): Molecular and cellular mechanisms of ectodomain shedding. In: Anatomical record (Hoboken, N.J.: 2007) 293 (6), p. 925-937.

He, Q.; McLellan, H.; Boevink, P. C.; Sadanandom, A.; Xie, C.; Birch, P. R. J.; Tian, Z. (2015): Ubox E3 ubiquitin ligase PUB17 acts in the nucleus to promote specific immune pathways triggered by Phytophthora infestans. In: Journal of experimental botany 66 (11), p. 31893199 .

Heese, A.; Hann, D. R.; Gimenez-Ibanez, S.; Jones, A. M. E.; He, K.; Li, J. et al. (2007): The receptor-like kinase SERK3/BAK1 is a central regulator of innate immunity in plants. In: Proceedings of the National Academy of Sciences of the United States of America 104 (29), p. 12217-12222.

Heigwer, F.; Kerr, G.; Boutros, M. (2014): E-CRISP: fast CRISPR target site identification. In: Nature methods 11 (2), p. 122-123.

Hellens, R.; Mullineaux, P.; Klee, H. (2000): Technical Focus: A guide to Agrobacterium binary Ti vectors. In: Trends in plant science 5 (10), p. 446-451.

Hemetsberger, C.; Herrberger, C.; Zechmann, B.; Hillmer, M.; Doehlemann, G. (2012): The Ustilago maydis effector Pep1 suppresses plant immunity by inhibition of host peroxidase activity. In: PLoS pathogens 8 (5), e1002684. 
Hong, Z.; Jin, H.; Tzfira, T.; Li, J. (2008): Multiple mechanism-mediated retention of a defective brassinosteroid receptor in the endoplasmic reticulum of Arabidopsis. In: The Plant cell 20 (12), p. 3418-3429.

Houston, K.; Tucker, M. R.; Chowdhury, J.; Shirley, N.; Little, A. (2016): The Plant Cell Wall: A Complex and Dynamic Structure As Revealed by the Responses of Genes under Stress Conditions. In: Frontiers in plant science 7, p. 984.

Howell, S. H. (2013): Endoplasmic reticulum stress responses in plants. In: Annual review of plant biology 64, p. 477-499.

Huang, P.-Y.; Yeh, Y.-H.; Liu, A.-C.; Cheng, C.-P.; Zimmerli, L. (2014): The Arabidopsis LecRKVI.2 associates with the pattern-recognition receptor FLS2 and primes Nicotiana benthamiana pattern-triggered immunity. In: The Plant journal: for cell and molecular biology 79 (2), p. $243-255$.

Huffaker, A.; Pearce, G.; Ryan, C. A. (2006): An endogenous peptide signal in Arabidopsis activates components of the innate immune response. In: Proceedings of the National Academy of Sciences of the United States of America 103 (26), p. 10098-10103.

Huse, M.; Kuriyan, J. (2002): The Conformational Plasticity of Protein Kinases. In: Cell 109 (3), p. 275-282.

Idänheimo, N.; Gauthier, A.; Salojärvi, J.; Siligato, R.; Brosché, M.; Kollist, H. et al. (2014): The Arabidopsis thaliana cysteine-rich receptor-like kinases CRK6 and CRK7 protect against apoplastic oxidative stress. In: Biochemical and biophysical research communications 445 (2), p. 457-462.

Ishikawa, K.; Yamaguchi, K.; Sakamoto, K.; Yoshimura, S.; Inoue, K.; Tsuge, S. et al. (2014): Bacterial effector modulation of host E3 ligase activity suppresses PAMP-triggered immunity in rice. In: Nature communications 5, p. 5430.

Jin, Q.; Thilmony, R.; Zwiesler-Vollick, J.; He, S.-Y. (2003): Type III protein secretion in Pseudomonas syringae. In: Microbes and infection 5 (4), p. 301-310.

Jirage, D.; Tootle, T. L.; Reuber, T. L.; Frost, L. N.; Feys, B. J.; Parker, J. E. et al. (1999): Arabidopsis thaliana PAD4 encodes a lipase-like gene that is important for salicylic acid signaling. In: Proceedings of the National Academy of Sciences of the United States of America 96 (23), p. 13583-13588.

Jones, J. D. G.; Dangl, J. L. (2006): The plant immune system. In: Nature 444 (7117), p. 323-329.

Kadota, Y.; Sklenar, J.; Derbyshire, P.; Stransfeld, L.; Asai, S.; Ntoukakis, V. et al. (2014): Direct regulation of the NADPH oxidase RBOHD by the PRR-associated kinase BIK1 during plant immunity. In: Molecular cell 54 (1), p. 43-55.

Kaku, H.; Nishizawa, Y.; Ishii-Minami, N.; Akimoto-Tomiyama, C.; Dohmae, N.; Takio, K. et al. (2006): Plant cells recognize chitin fragments for defense signaling through a plasma membrane receptor. In: Proceedings of the National Academy of Sciences of the United States of America 103 (29), p. 11086-11091.

Kalinina, N. O.; Makarova, S.; Makhotenko, A.; Love, A. J.; Taliansky, M. (2018): The Multiple Functions of the Nucleolus in Plant Development, Disease and Stress Responses. In: Frontiers in plant science 9, p. 132.

Kankanala, P.; Czymmek, K.; Valent, B. (2007): Roles for rice membrane dynamics and plasmodesmata during biotrophic invasion by the blast fungus. In: The Plant cell 19 (2), p. 706-724.

Karlova, R.; Boeren, S.; Russinova, E.; Aker, J.; Vervoort, J.; de Vries, S. (2006): The Arabidopsis SOMATIC EMBRYOGENESIS RECEPTOR-LIKE KINASE1 protein complex includes BRASSINOSTEROID-INSENSITIVE1. In: The Plant cell 18 (3), p. 626-638. 
Kim, S. Y.; Shang, Y.; Joo, S.-H.; Kim, S.-K.; Nam, K. H. (2017): Overexpression of BAK1 causes salicylic acid accumulation and deregulation of cell death control genes. In: Biochemical and biophysical research communications 484 (4), p. 781-786.

Kimura, S.; Hunter, K.; Vaahtera, L.; Tran, H. C.; Citterico, M.; Vaattovaara, A. et al. (2020): CRK2 and C-terminal Phosphorylation of NADPH Oxidase RBOHD Regulate Reactive Oxygen Species Production in Arabidopsis. In: The Plant cell 32 (4), p. 1063-1080.

Kleinboelting, N.; Huep, G.; Kloetgen, A.; Viehoever, P.; Weisshaar, B. (2012): GABI-Kat Simple Search: new features of the Arabidopsis thaliana T-DNA mutant database. In: Nucleic acids research 40 (Database issue), D1211-5.

Koncz, C.; Schell, J. (1986): The promoter of TL-DNA gene 5 controls the tissue-specific expression of chimaeric genes carried by a novel type of Agrobacterium binary vector. In: Molec Gen Genet 204 (3), p. 383-396.

Kosugi, S.; Hasebe, M.; Tomita, M.; Yanagawa, H. (2009): Systematic identification of cell cycledependent yeast nucleocytoplasmic shuttling proteins by prediction of composite motifs. In: Proceedings of the National Academy of Sciences 106 (25), p. 10171-10176.

Kourelis, J.; Kaschani, F.; Grosse-Holz, F. M.; Homma, F.; Kaiser, M.; van der Hoorn, R. A. L. (2019): A homology-guided, genome-based proteome for improved proteomics in the alloploid Nicotiana benthamiana. In: BMC genomics 20 (1), p. 722.

Kouzai, Y.; Mochizuki, S.; Nakajima, K.; Desaki, Y.; Hayafune, M.; Miyazaki, H. et al. (2014): Targeted gene disruption of OsCERK1 reveals its indispensable role in chitin perception and involvement in the peptidoglycan response and immunity in rice. In: Molecular plant-microbe interactions: MPMI 27 (9), p. 975-982.

Krol, E.; Mentzel, T.; Chinchilla, D.; Boller, T.; Felix, G.; Kemmerling, B. et al. (2010): Perception of the Arabidopsis danger signal peptide 1 involves the pattern recognition receptor AtPEPR1 and its close homologue AtPEPR2. In: The Journal of biological chemistry 285 (18), p. 13471-13479.

Kunze, G.; Zipfel, C.; Robatzek, S.; Niehaus, K.; Boller, T.; Felix, G. (2004): The N terminus of bacterial elongation factor Tu elicits innate immunity in Arabidopsis plants. In: The Plant cell 16 (12), p. 3496-3507.

Lal, N. K.; Nagalakshmi, U.; Hurlburt, N. K.; Flores, R.; Bak, A.; Sone, P. et al. (2018): The Receptor-like Cytoplasmic Kinase BIK1 Localizes to the Nucleus and Regulates Defense Hormone Expression during Plant Innate Immunity. In: Cell host \& microbe 23 (4), 485-497.e5.

Lapin, D.; Kovacova, V.; Sun, X.; Dongus, J. A.; Bhandari, D.; von Born, P. et al. (2019): A Coevolved EDS1-SAG101-NRG1 Module Mediates Cell Death Signaling by TIR-Domain Immune Receptors. In: The Plant cell 31 (10), p. 2430-2455.

Lee, D. S.; Kim, Y. C.; Kwon, S. J.; Ryu, C.-M.; Park, O. K. (2017): The Arabidopsis Cysteine-Rich Receptor-Like Kinase CRK36 Regulates Immunity through Interaction with the Cytoplasmic Kinase BIK1. In: Frontiers in plant science 8, p. 1856.

Lee, J.-Y.; Lu, H. (2011): Plasmodesmata: the battleground against intruders. In: Trends in plant science 16 (4), p. 201-210.

Lee, J.-Y.; Wang, X.; Cui, W.; Sager, R.; Modla, S.; Czymmek, K. et al. (2011): A plasmodesmatalocalized protein mediates crosstalk between cell-to-cell communication and innate immunity in Arabidopsis. In: The Plant cell 23 (9), p. 3353-3373.

Lee, S.-W.; Han, S.-W.; Sririyanum, M.; Park, C.-J.; Seo, Y.-S.; Ronald, P. C. (2009): A type I- secreted, sulfated peptide triggers XA21-mediated innate immunity. In: Science (New York, N.Y.) 326(5954), p. 850-853.

Lee, Y. R.; Assmann, S. M. (1999): Arabidopsis thaliana 'extra-large GTP-binding protein' (AtXLG1): a new class of G-protein. In: Plant molecular biology 40 (1), p. 55-64. 
Lenormand, C.; Spiegelhalter, C.; Cinquin, B.; Bardin, S.; Bausinger, H.; Angénieux, C. et al. (2013): Birbeck granule-like "organized smooth endoplasmic reticulum" resulting from the expression of a cytoplasmic YFP-tagged langerin. In: PloS one 8 (4), e60813.

Leonelli, L.; Pelton, J; Schoeffler, A.; Dahlbeck, D.; Berger, J.; Wemmer, D. E.; Staskawicz, B. (2011): Structural elucidation and functional characterization of the Hyaloperonospora arabidopsidis effector protein ATR13. In: PLoS pathogens 7 (12), e1002428.

Lerouge, P.; Roche, P.; Faucher, C.; Maillet, F.; Truchet, G.; Promé, J. C.; Dénarié, J. (1990): Symbiotic host-specificity of Rhizobium meliloti is determined by a sulphated and acylated glucosamine oligosaccharide signal. In: Nature 344 (6268), p. 781-784.

Le Roux, C.; Huet, G.; Jauneau, A.; Camborde, L.; Trémousaygue, D.; Kraut, A. et al. (2015): A receptor pair with an integrated decoy converts pathogen disabling of transcription factors to immunity. In: Cell 161 (5), p. 1074-1088.

Lesage, G.; Bussey, H. (2006): Cell wall assembly in Saccharomyces cerevisiae. In: Microbiology and molecular biology reviews: MMBR 70 (2), p. 317-343.

Letunic, I.; Bork, P. (2018): 20 years of the SMART protein domain annotation resource. In: Nucleic acids research 46 (D1), D493-D496.

Li, X.; Lin, H.; Zhang, W.; Zou, Y.; Zhang, J.; Tang, X.; Zhou, J.-M. (2005): Flagellin induces innate immunity in nonhost interactions that is suppressed by Pseudomonas syringae effectors. In: Proceedings of the National Academy of Sciences of the United States of America 102 (36), p.12990-12995.

Liang, X.; Zhou, J.-M. (2018): Receptor-Like Cytoplasmic Kinases: Central Players in Plant Receptor Kinase-Mediated Signaling. In: Annual review of plant biology 69, p. 267-299.

Liang, Y.; Cao, Y.; Tanaka, K.; Thibivilliers, S.; Wan, J.; Choi, J. et al. (2013): Nonlegumes respond to rhizobial Nod factors by suppressing the innate immune response. In: Science (New York, N.Y.) 341 (6152), p. 1384-1387.

Liebrand, T. W. H.; van den Burg, H. A.; Joosten, M. H. A. J. (2014): Two for all: receptor-associated kinases SOBIR1 and BAK1. In: Trends in plant science 19 (2), p. 123-132.

Limpens, E.; Franken, C.; Smit, P.; Willemse, J.; Bisseling, T.; Geurts, R. (2003): LysM domain receptor kinases regulating rhizobial Nod factor-induced infection. In: Science (New York, N.Y.) 302 (5645), p. 630-633.

Lin, W.; Li, B.; Lu, D.; Chen, S.; Zhu, N.; He, P.; Shan, L. (2014): Tyrosine phosphorylation of protein kinase complex BAK1/BIK1 mediates Arabidopsis innate immunity. In: Proceedings of the National Academy of Sciences 111 (9), p. 3632-3637.

Lipka, V.; Dittgen, J.; Bednarek, P.; Bhat, R.; Wiermer, M.; Stein, M. et al. (2005): Pre- and postinvasion defenses both contribute to nonhost resistance in Arabidopsis. In: Science (New York, N.Y.) 310 (5751), p. 1180-1183.

Liu, B.; Li, J.-F.; Ao, Y.; Qu, J.; Li, Z.; Su, J. et al. (2012b): Lysin motif-containing proteins LYP4 and LYP6 play dual roles in peptidoglycan and chitin perception in rice innate immunity. In: The Plant cell 24 (8), p. 3406-3419.

Liu, H.; Ding, Y.; Zhou, Y.; Jin, W.; Xie, K.; Chen, L.-L. (2017a): CRISPR-P 2.0: An Improved CRISPR-Cas9 Tool for Genome Editing in Plants. In: Molecular plant 10 (3), p. 530-532.

Liu, J.; Chen, S.; Chen, L.; Zhou, Q.; Wang, M.; Feng, D. et al. (2017b): BIK1 cooperates with BAK1 to regulate constitutive immunity and cell death in Arabidopsis. In: Journal of integrative plant biology 59 (4), p. 234-239.

Liu, T.; Liu, Z.; Song, C.; Hu, Y.; Han, Z.; She, J. et al. (2012a): Chitin-induced dimerization activates a plant immune receptor. In: Science (New York, N.Y.) 336 (6085), p. 1160-1164. 
Liu, Z.; Wu, Y.; Yang, F.; Zhang, Y.; Chen, S.; Xie, Q. et al. (2013): BIK1 interacts with PEPRs to mediate ethylene-induced immunity. In: Proceedings of the National Academy of Sciences of the United States of America 110 (15), p. 6205-6210.

Lorković, Z. J.; Wieczorek Kirk, D. A.; Lambermon, M. H. L; Filipowicz, W. (2000): Pre-mRNA splicing in higher plants. In: Trends in plant science 5 (4), p. 160-167.

Lovering, A. L.; Safadi, S. S.; Strynadka, N. C. J. (2012): Structural perspective of peptidoglycan biosynthesis and assembly. In: Annual review of biochemistry 81, p. 451-478.

Lozano-Durán, R.; Bourdais, G.; He, S. Y.; Robatzek, S. (2014): The bacterial effector HopM1 suppresses PAMP-triggered oxidative burst and stomatal immunity. In: The New phytologist 202 (1), p. 259-269.

Lu, D.; Wu, S.; Gao, X.; Zhang, Y.; Shan, L.; He, P. (2010): A receptor-like cytoplasmic kinase, BIK1, associates with a flagellin receptor complex to initiate plant innate immunity. In: Proceedings of the National Academy of Sciences of the United States of America 107 (1), p. 496-501.

Ma, L.-S.; Wang, L.; Trippel, C.; Mendoza-Mendoza, A.; Ullmann, S.; Moretti, M. et al. (2018): The Ustilago maydis repetitive effector Rsp3 blocks the antifungal activity of mannose-binding maize proteins. In: Nature communications 9 (1), p. 1711.

Ma, X.; Xu, G.; He, P.; Shan, Li. (2016): SERKing Coreceptors for Receptors. In: Trends in plant science 21 (12), p. 1017-1033.

Macho, A. P.; Schwessinger, B.; Ntoukakis, V.; Brutus, A.; Segonzac, C.; Roy, S. et al. (2014): A bacterial tyrosine phosphatase inhibits plant pattern recognition receptor activation. In: $\mathrm{Sci}$ ence (New York, N.Y.) 343 (6178), p. 1509-1512.

Macho, A. P.; Zipfel, C. (2014): Plant PRRs and the activation of innate immune signaling. In: $\quad M o-$ lecular cell 54 (2), p. 263-272.

Madeira, F.; Park, Y. M.; Lee, J.; Buso, N.; Gur, T.; Madhusoodanan, N. et al. (2019): The EMBLEBI search and sequence analysis tools APIs in 2019. In: Nucleic acids research 47 (W1), W636-W641.

Madsen, E. B.; Antolín-Llovera, M.; Grossmann, C.; Ye, J.; Vieweg, S.; Broghammer, A. et al. (2011): Autophosphorylation is essential for the in vivo function of the Lotus japonicus Nod factor receptor 1 and receptor-mediated signalling in cooperation with Nod factor receptor 5. In: The Plant journal: for cell and molecular biology 65 (3), p. 404-417.

Malamy, J.; Carr, J. P.; Klessig, D. F.; Raskin, I. (1990): Salicylic Acid: a likely endogenous signal in the resistance response of tobacco to viral infection. In: Science (New York, N.Y.) 250 (4983), p. 1002-1004.

Marshall, A.; Aalen, R. B.; Audenaert, D.; Beeckman, T.; Broadley, M. R.; Butenko, M. A. et al. (2012): Tackling drought stress: receptor-like kinases present new approaches. In: The Plant cell 24 (6), p. 2262-2278.

Maruta, N.; Trusov, Y.; Brenya, E.; Parekh, U.; Botella, J. R. (2015): Membrane-localized extralarge $\mathrm{G}$ proteins and $\mathrm{Gbg}$ of the heterotrimeric $\mathrm{G}$ proteins form functional complexes engaged in plant immunity in Arabidopsis. In: Plant physiology 167 (3), p. 1004-1016.

Maule, A. J.; Benitez-Alfonso, Y.; Faulkner, C. (2011): Plasmodesmata-membrane tunnels with attitude. In: Current opinion in plant biology 14 (6), p. 683-690.

Mélida, H.; Sopeña-Torres, S.; Bacete, L.; Garrido-Arandia, M.; Jordá, L.; López, G. et al. (2018): Non-branched $\beta$-1,3-glucan oligosaccharides trigger immune responses in Arabidopsis. In: The Plant journal: for cell and molecular biology 93 (1), p. 34-49.

Meusel, C. (2016): Analysis of CERK1 ectodomain shedding and the role of XLG2 in cerk1-4 cell death execution. 
Miya, A.; Albert, P.; Shinya, T.; Desaki, Y.; Ichimura, K.; Shirasu, K. et al. (2007): CERK1, a LysM receptor kinase, is essential for chitin elicitor signaling in Arabidopsis. In: Proceedings of the National Academy of Sciences of the United States of America 104 (49), p. 19613-19618.

Miyakawa, T.; Hatano, K.; Miyauchi, Y.; Suwa, Y.; Sawano, Y.; Tanokura, M. (2014): A secreted protein with plant-specific cysteine-rich motif functions as a mannose-binding lectin that exhibits antifungal activity. In: Plant physiology 166 (2), p. 766-778.

Miyakawa, T.; Miyazono, K.; Sawano, Y.; Hatano, K.; Tanokura, M. (2009): Crystal structure of ginkbilobin-2 with homology to the extracellular domain of plant cysteine-rich receptor-like kinases. In: Proteins 77 (1), p. 247-251.

Moling, S.; Pietraszewska-Bogiel, A.; Postma, M.; Fedorova, E.; Hink, M. A.; Limpens, E. et al. (2014): Nod factor receptors form heteromeric complexes and are essential for intracellular infection in medicago nodules. In: The Plant cell 26 (10), p. 4188-4199.

Müller, A. N.; Ziemann, S.; Treitschke, S.; Aßmann, D.; Doehlemann, G. (2013): Compatibility in the Ustilago maydis-maize interaction requires inhibition of host cysteine proteases by the fungal effector Pit2. In: PLoS pathogens 9 (2), e1003177.

Muzzarelli, R. A. A. (1978): Chitin. 1. ed. 1977, repr. Oxford: Pergamon Press.

Naito, Y.; Hino, K.; Bono, H.; Ui-Tei, K. (2015): CRISPRdirect: software for designing CRISPR/Cas guide RNA with reduced off-target sites. In: Bioinformatics (Oxford, England) 31 (7), p. $1120-1123$.

Nakagawa, T.; Kaku, H.; Shimoda, Y.; Sugiyama, A.; Shimamura, M.; Takanashi, K. et al. (2011): From defense to symbiosis: limited alterations in the kinase domain of LysM receptor-like kinases are crucial for evolution of legume-Rhizobium symbiosis. In: The Plant journal: for cell and molecular biology 65 (2), p. 169-180.

Nelson, B. K.; Cai, X.; Nebenführ, A. (2007): A multicolored set of in vivo organelle markers for co-localization studies in Arabidopsis and other plants. In: The Plant journal: for cell and molecular biology 51 (6), p. 1126-1136.

Neubauer, M.; Serrano, I.; Rodibaugh, N.; Bhandari, D. D.; Bautor, J.; Parker, J. E.; Innes, R. W. (2020): Arabidopsis EDR1 Protein Kinase Regulates the Association of EDS1 and PAD4 to Inhibit Cell Death. In: Molecular plant-microbe interactions: MPMI 33 (4), p. 693-703.

Noman, A.; Aqeel, M.; Lou, Y. (2019): PRRs and NB-LRRs: From Signal Perception to Activation of Plant Innate Immunity. In: International journal of molecular sciences 20 (8).

Obayashi, T.; Aoki, Y.; Tadaka, S.; Kagaya, Y.; Kinoshita, K. (2018): ATTED-II in 2018: A Plant Coexpression Database Based on Investigation of the Statistical Property of the Mutual Rank Index. In: Plant \& cell physiology 59 (1), e3.

O'Connell, R. J.; Panstruga, R. (2006): Tête à tête inside a plant cell: establishing compatibility between plants and biotrophic fungi and oomycetes. In: The New phytologist 171 (4), p. 699718 .

Ohtake, Y.; Takahashi, T.; Komeda, Y. (2000): Salicylic acid induces the expression of a number of receptor-like kinase genes in Arabidopsis thaliana. In: Plant \& cell physiology 41 (9), p. $1038-1044$.

Oldroyd, G. E. D.; Downie, J. A. (2008): Coordinating nodule morphogenesis with rhizobial infection in legumes. In: Annual review of plant biology 59, p. 519-546.

Otero, J. H.; Lizák, B.; Hendershot, L. M. (2010): Life and death of a BiP substrate. In: Seminars in cell \& developmental biology 21 (5), p. 472-478.

Panstruga, R.; Dodds, P. N. (2009): Terrific protein traffic: the mystery of effector protein delivery by filamentous plant pathogens. In: Science (New York, N.Y.) 324 (5928), p. 748-750. 
Park, C.-H.; Chen, S.; Shirsekar, G.; Zhou, B; Khang, C. H.; Songkumarn, P. et al. (2012): The Magnaporthe oryzae effector AvrPiz-t targets the RING E3 ubiquitin ligase APIP6 to suppress pathogen-associated molecular pattern-triggered immunity in rice. In: The Plant cell 24 (11), p. 4748-4762.

Petre, B.; Kamoun, S. (2014): How do filamentous pathogens deliver effector proteins into plant cells? In: PLoS biology 12 (2), e1001801.

Petutschnig, E. K.; Jones, A. M. E.; Serazetdinova, L.; Lipka, U.; Lipka, V. (2010): The lysin motif receptor-like kinase (LysM-RLK) CERK1 is a major chitin-binding protein in Arabidopsis thaliana and subject to chitin-induced phosphorylation. In: The Journal of biological chemistry 285 (37), p. 28902-28911.

Petutschnig, E. K.; Stolze, M.; Lipka, U.; Kopischke, M.; Horlacher, J.; Valerius, O. et al. (2014): A novel Arabidopsis CHITIN ELICITOR RECEPTOR KINASE 1 (CERK1) mutant with enhanced pathogen-induced cell death and altered receptor processing. In: The New phytologist 204 (4), p. 955-967.

Pfund, C.; Tans-Kersten, J.; Dunning, F. M.; Alonso, J. M.; Ecker, J. R.; Allen, C.; Bent, A. F. (2004): Flagellin is not a major defense elicitor in Ralstonia solanacearum cells or extracts applied to Arabidopsis thaliana. In: Molecular plant-microbe interactions: MPMI 17 (6), p. 696-706.

Pontes, O.; Vitins, A.; Ream, T. S.; Hong, E.; Pikaard, C. S.; Costa-Nunes, P. (2013): Intersection of small RNA pathways in Arabidopsis thaliana sub-nuclear domains. In: PloS one 8 (6), e65652.

Postel, S.; Kemmerling, B. (2009): Plant systems for recognition of pathogen-associated molecular patterns. In: Seminars in cell \& developmental biology 20 (9), p. 1025-1031.

Postel, S.; Küfner, I.; Beuter, C.; Mazzotta, S.; Schwedt, A.; Borlotti, A. et al. (2010): The multifunctional leucine-rich repeat receptor kinase BAK1 is implicated in Arabidopsis development and immunity. In: European journal of cell biology 89 (2-3), p. 169-174.

Pruitt, R. N.; Schwessinger, B.; Joe, A.; Thomas, N.; Liu, F.; Albert, M. et al. (2015): The rice immune receptor XA21 recognizes a tyrosine-sulfated protein from a Gram-negative bacterium. In: Science advances 1 (6), e1500245.

Qi, Z.; Verma, R.; Gehring, C.; Yamaguchi, Y.; Zhao, Y.; Ryan, C. A.; Berkowitz, G. A. (2010): $\mathrm{Ca}^{2+}$ signaling by plant Arabidopsis thaliana Pep peptides depends on AtPepR1, a receptor with guanylyl cyclase activity, and cGMP-activated Ca2+ channels. In: Proceedings of the National Academy of Sciences of the United States of America 107 (49), p. 21193-21198.

Radutoiu, S.; Madsen, L. H.; Madsen, E. B.; Felle, H. H.; Umehara, Y.; Grønlund, M. et al. (2003): Plant recognition of symbiotic bacteria requires two LysM receptor-like kinases. In: Nature 425 (6958), p. 585-592.

Rappsilber, J.; Ishihama, Y.; Mann, M. (2003): Stop and go extraction tips for matrix-assisted laser desorption/ionization, nanoelectrospray, and LC/MS sample pretreatment in proteomics. In: Analytical chemistry 75 (3), p. 663-670.

Rappsilber, J.; Mann, M.; Ishihama, Y. (2007): Protocol for micro-purification, enrichment, pre-fractionation and storage of peptides for proteomics using StageTips. In: Nature protocols 2 (8), p. 1896-1906.

Rasmussen, M. W.; Roux, M.; Petersen, M.; Mundy, J. (2012): MAP Kinase Cascades in Arabidopsis Innate Immunity. In: Frontiers in plant science 3, p. 169.

Rey, T.; Nars, A.; Bonhomme, M.; Bottin, A.; Huguet, S.; Balzergue, S. et al. (2013): NFP, a LysM protein controlling Nod factor perception, also intervenes in Medicago truncatula resistance to pathogens. In: The New phytologist 198 (3), p. 875-886. 
Round, E. K.; Flowers, S. K.; Richards, E. J. (1997): Arabidopsis thaliana centromere regions: genetic map positions and repetitive DNA structure. In: Genome research 7 (11), p. 1045-1053.

Roux, M.; Schwessinger, B.; Albrecht, C.; Chinchilla, D.; Jones, A.; Holton, N. et al. (2011): The Arabidopsis leucine-rich repeat receptor-like kinases BAK1/SERK3 and BKK1/SERK4 are required for innate immunity to hemibiotrophic and biotrophic pathogens. In: The Plant cell 23 (6), p. 2440-2455.

Rushton, P. J.; Somssich, I. E.; Ringler, P.; Shen, Q. J. (2010): WRKY transcription factors. In: Trends in plant science 15 (5), p. 247-258.

Sager, R. E.; Lee, J.-Y. (2018): Plasmodesmata at a glance. In: Journal of cell science 131 (11).

Sarris, P. F.; Duxbury, Z.; Huh, S. U.; Ma, Y.; Segonzac, C.; Sklenar, J. et al. (2015): A Plant Immune Receptor Detects Pathogen Effectors that Target WRKY Transcription Factors. In: Cell 161 (5), p. 1089-1100.

Sawano, Y.; Miyakawa, T; Yamazaki, H.; Tanokura, M.; Hatano, K. (2007): Purification, characterization, and molecular gene cloning of an antifungal protein from Ginkgo biloba seeds. In: Biological chemistry 388 (3), p. 273-280.

Schulze, B.; Mentzel, T.; Jehle, A. K.; Mueller, K.; Beeler, S.; Boller, T. et al. (2010): Rapid heteromerization and phosphorylation of ligand-activated plant transmembrane receptors and their associated kinase BAK1. In: The Journal of biological chemistry 285 (13), p. 9444-9451.

Schwacke, R.; Schneider, A.; van der Graaff, E.; Fischer, K.; Catoni, E.; Desimone, M. et al. (2003): ARAMEMNON, a novel database for Arabidopsis integral membrane proteins. In: Plant physiology 131 (1), p. 16-26.

Scott, M. S.; Boisvert, F.-M.; McDowall, M. D.; Lamond, A. I.; Barton, G. J. (2010): Characterization and prediction of protein nucleolar localization sequences. In: Nucleic acids research 38 (21), p. 7388-7399.

Selin, C.; de Kievit, T. R.; Belmonte, M. F.; Fernando, W. G. D. (2016): Elucidating the Role of Effectors in Plant-Fungal Interactions: Progress and Challenges. In: Frontiers in microbiology 7, p. 600 .

Seyfferth, C.; Tsuda, K. (2014): Salicylic acid signal transduction: the initiation of biosynthesis, perception and transcriptional reprogramming. In: Frontiers in Plant Science 5, p. 697.

Shimizu, T.; Nakano, T.; Takamizawa, D.; Desaki, Y.; Ishii-Minami, N.; Nishizawa, Y. et al. (2010): Two LysM receptor molecules, CEBiP and OsCERK1, cooperatively regulate chitin elicitor signaling in rice. In: The Plant journal: for cell and molecular biology 64 (2), p. 204-214.

Shinya, T.; Motoyama, N.; Ikeda, A.; Wada, M.; Kamiya, K.; Hayafune, M. et al. (2012): Functional characterization of CEBiP and CERK1 homologs in arabidopsis and rice reveals the presence of different chitin receptor systems in plants. In: Plant \& cell physiology 53 (10), p. 16961706.

Shinya, T.; Nakagawa, T.; Kaku, H.; Shibuya, N. (2015): Chitin-mediated plant-fungal interactions: catching, hiding and handshaking. In: Current opinion in plant biology 26, p. 64-71.

Shiu, S. H.; Bleecker, A. B. (2001): Plant receptor-like kinase gene family: diversity, function, and signaling. In: Science's STKE: signal transduction knowledge environment 2001 (113), re22.

Shiu, S. H.; Bleecker, A. B. (2003): Expansion of the receptor-like kinase/Pelle gene family and re ceptor-like proteins in Arabidopsis. In: Plant physiology 132 (2), p. 530-543.

Singh, P.; Kuo, Y.-C.; Mishra, S.; Tsai, C.-H.; Chien, C.-C.; Chen, C.-W. et al. (2012): The lectin receptor kinase-VI.2 is required for priming and positively regulates Arabidopsis patterntriggered immunity. In: The Plant cell 24 (3), p. 1256-1270. 
Smit, P.; Limpens, E.; Geurts, R.; Fedorova, E.; Dolgikh, E.; Gough, C.; Bisseling, T. (2007): Medicago LYK3, an entry receptor in rhizobial nodulation factor signaling. In: Plant physiology 145 (1), p. 183-191.

Snapp, E. L.; Hegde, R. S.; Francolini, M.; Lombardo, F.; Colombo, S.; Pedrazzini, E. et al. (2003): Formation of stacked ER cisternae by low affinity protein interactions. In: The Journal of cell biology 163 (2), p. 257-269.

Strasser, R. (2016): Plant protein glycosylation. In: Glycobiology 26 (9), p. 926-939.

Su, W.; Liu, Y.; Xia, Y.; Hong, Z.; Li, J. (2011): Conserved endoplasmic reticulum-associated degradation system to eliminate mutated receptor-like kinases in Arabidopsis. In: Proceedings of the National Academy of Sciences 108 (2), p. 870-875.

Sun, W.; Dunning, F. M.; Pfund, C.; Weingarten, R.; Bent, A. F. (2006): Within-species flagellin polymorphism in Xanthomonas campestris pv. campestris and its impact on elicitation of Arabidopsis FLAGELLIN SENSING2-dependent defenses. In: The Plant cell 18 (3), p. 764-779.

Sun, Y.; Han, Z.; Tang, J.; Hu, Z.; Chai, C.; Zhou, B.; Chai, J (2013): Structure reveals that BAK1 as a co-receptor recognizes the BRI1-bound brassinolide. In: Cell research 23 (11), p. 13261329.

Tanaka, S.; Brefort, T.; Neidig, N.; Djamei, A.; Kahnt, J.; Vermerris, W. et al. (2014): A secreted Ustilago maydis effector promotes virulence by targeting anthocyanin biosynthesis in maize. In: eLife 3, e01355.

Tanaka, H.; Osakabe, Y.; Katsura, S.; Mizuno, S.; Maruyama, K.; Kusakabe, K. et al. (2012): Abiotic stress-inducible receptor-like kinases negatively control ABA signaling in Arabidopsis. In: The Plant journal: for cell and molecular biology 70 (4), p. 599-613.

Taylor, S. S.; Radzio-Andzelm, E. (1994): Three protein kinase structures define a common motif. In: Structure (London, England: 1993) 2 (5), p. 345-355.

Thomas, C. L.; Bayer, E. M.; Ritzenthaler, C.; Fernandez-Calvino, L.; Maule, A. J. (2008): Specific targeting of a plasmodesmal protein affecting cell-to-cell communication. In: PLoS biology 6 (1),e7.

Thordal-Christensen, Hans (2020): A holistic view on plant effector-triggered immunity presented as an iceberg model. In: Cellular and molecular life sciences: CMLS. DOI: 10.1007/s00018020-03515-w.

Tilsner, J.; Linnik, O.; Louveaux, M.; Roberts, I. M.; Chapman, S. N.; Oparka, K. J. (2013): Replication and trafficking of a plant virus are coupled at the entrances of plasmodesmata. In: The Journal of cell biology 201 (7), p. 981-995.

Torres, M. A.; Jones, J. D. G.; Dangl, J. L. (2005): Pathogen-induced, NADPH oxidase-derived reactive oxygen intermediates suppress spread of cell death in Arabidopsis thaliana. In: Nature genetics 37 (10), p. 1130-1134.

Trusov, Y.; Botella, J. R. (2016): Plant G-Proteins Come of Age: Breaking the Bond with Animal Models. In: Frontiers in chemistry 4, p. 24.

Ueki, S.; Citovsky, V. (2011): To gate, or not to gate: regulatory mechanisms for intercellular protein transport and virus movement in plants. In: Molecular plant 4 (5), p. 782-793.

Ülker, B.; Peiter, E.; Dixon, D. P.; Moffat, C.; Capper, R.; Bouché, N. et al. (2008): Getting the most out of publicly available T-DNA insertion lines. In: The Plant journal: for cell and molecular biology 56 (4), p. 665-677.

Vaattovaara, A.; Brandt, B.; Rajaraman, S.; Safronov, O.; Veidenberg, A.; Luklová, M. et al. (2019): Mechanistic insights into the evolution of DUF26-containing proteins in land plants. In: Communications biology 2, p. 56. 
van Eerde, A.; Grahn, E. M.; Winter, H. C.; Goldstein, I. J.; Krengel, U. (2015): Atomic-resolution structure of the $\alpha$-galactosyl binding Lyophyllum decastes lectin reveals a new protein family found in both fungi and plants. In: Glycobiology 25 (5), p. 492-501.

van der Hoorn, R. A. L.; Kamoun, S. (2008): From Guard to Decoy: a new model for perception of plant pathogen effectors. In: The Plant cell 20 (8), p. 2009-2017.

Vaucheret, H.; Béclin, C.; Fagard, M. (2001): Post-transcriptional gene silencing in plants. In: Journal of cell science 114 (Pt 17), p. 3083-3091.

Via, V. D.; Zanetti, M. E.; Blanco, F. (2016): How legumes recognize rhizobia. In: Plant signaling \& behavior 11 (2), e1120396.

Wagner, S.; Stuttmann, J.; Rietz, S.; Guerois, R.; Brunstein, E.; Bautor, J. et al. (2013): Structural basis for signaling by exclusive EDS1 heteromeric complexes with SAG101 or PAD4 in plant innate immunity. In: Cell host \& microbe 14 (6), p. 619-630.

Wan, J.; Tanaka, K.; Zhang, X.-C.; Son, G. H.; Brechenmacher, L.; Nguyen, T. H. N.; Stacey, G. (2012): LYK4, a lysin motif receptor-like kinase, is important for chitin signaling and plant innate immunity in Arabidopsis. In: Plant physiology 160 (1), p. 396-406.

Wan, J.; Zhang, X.-C.; Neece, D.; Ramonell, K. M.; Clough, S.; Kim, S.-Y. et al. (2008): A LysM receptor-like kinase plays a critical role in chitin signaling and fungal resistance in Arabidopsis. In: The Plant cell 20 (2), p. 471-481.

Wang, Jizong; Hu, Meijuan; Wang, Jia; Qi, Jinfeng; Han, Zhifu; Wang, Guoxun et al. (2019): Reconstitution and structure of a plant NLR resistosome conferring immunity. In: Science (New York, N.Y.) 364 (6435).

Wang, Jizong; Wang, Jia; Hu, Meijuan; Wu, Shan; Qi, Jinfeng; Wang, Guoxun et al. (2019): Ligandtriggered allosteric ADP release primes a plant NLR complex. In: Science (New York, N.Y.) 364(6435).

Wiermer, M.; Feys, B. J.; Parker, J. E. (2005): Plant immunity: the EDS1 regulatory node. In: Current opinion in plant biology 8 (4), p. 383-389.

Wilkie, A. O. M. (2006): Dominance and Recessivity. In: John Wiley \&. Sons Ltd (Hg.): Encyclopedia of life sciences. London, New York, Vols. 21-32, Chichester, West Sussex, U.K.: Nature Pub. Group; Wiley, p. 1-10.

Willmann, R.; Lajunen, H. M.; Erbs, G.; Newman, M.-A.; Kolb, D.; Tsuda, K. et al. (2011): Arabidopsis lysin-motif proteins LYM1 LYM3 CERK1 mediate bacterial peptidoglycan sensing and immunity to bacterial infection. In: Proceedings of the National Academy of Sciences ofthe United States of America 108 (49), p. 19824-19829.

Winter, D.; Vinegar, B.; Nahal, H.; Ammar, R.; Wilson, G. V.; Provart, N. J. (2007): An "Electronic Fluorescent Pictograph" browser for exploring and analyzing large-scale biological data sets. In: PloS one 2 (8), e 718.

Wong, J. E. M. M.; Nadzieja, M.; Madsen, L. H.; Bücherl, C. A.; Dam, S.; Sandal, N. N. et al. (2019): A Lotus japonicus cytoplasmic kinase connects Nod factor perception by the NFR5 LysM receptor to nodulation. In: Proceedings of the National Academy of Sciences of the United States of America 116 (28), p. 14339-14348.

Wrzaczek, M.; Brosché, M.; Salojärvi, J.; Kangasjärvi, S.; Idänheimo, N.; Mersmann, S. et al. (2010): Transcriptional regulation of the CRK/DUF26 group of receptor-like protein kinases by ozone and plant hormones in Arabidopsis. In: BMC plant biology 10, p. 95.

Wu, C.-H.; Derevnina, L.; Kamoun, S. (2018): Receptor networks underpin plant immunity. In: Sci ence (New York, N.Y.) 360 (6395), p. 1300-1301. 
Yadeta, K. A.; Elmore, J. M.; Creer, A. Y.; Feng, B.; Franco, J. Y.; Rufian, J. S. et al. (2017): A Cysteine-Rich Protein Kinase Associates with a Membrane Immune Complex and the Cysteine Residues Are Required for Cell Death. In: Plant physiology 173 (1), p. 771-787.

Yamaguchi, Y.; Huffaker, A.; Bryan, A. C.; Tax, F. E.; Ryan, C. A. (2010): PEPR2 is a second receptor for the Pep1 and Pep2 peptides and contributes to defense responses in Arabidopsis. In: The Plant cell 22 (2), p. 508-522.

Yeh, Y.-H.; Chang, Y.-H.; Huang, P.-Y.; Huang, J.-B.; Zimmerli, L. (2015): Enhanced Arabidopsis pattern-triggered immunity by overexpression of cysteine-rich receptor-like kinases. In: Frontiers in plant science 6, p. 322.

Yu, X.; Feng, B.; He, P.; Shan, L. (2017): From Chaos to Harmony: Responses and Signaling upon Microbial Pattern Recognition. In: Annual review of phytopathology 55, p. 109-137.

Zambryski, P.; Crawford, K. (2000): Plasmodesmata: gatekeepers for cell-to-cell transport of developmental signals in plants. In: Annual review of cell and developmental biology 16, p. 393421.

Zhang, J.; Li, W.; Xiang, T.; Liu, Z.; Laluk, K.; Ding, X. et al. (2010): Receptor-like cytoplasmic kinases integrate signaling from multiple plant immune receptors and are targeted by a Pseudomonas syringae effector. In: Cell host \& microbe 7 (4), p. 290-301.

Zhang, J.; Shao, F.; Li, Y.; Cui, H.; Chen, L.; Li, H. et al. (2007): A Pseudomonas syringae effector inactivates MAPKs to suppress PAMP-induced immunity in plants. In: Cell host \& microbe 1 (3), p. 175-185.

Zhang, P.; Li, K.; Yang, G.; Xia, C.; Polston, J. E.; Li, G. et al. (2017): Cytotoxic protein from the mushroom Coprinus comatus possesses a unique mode for glycan binding and specificity. In: Proceedings of the National Academy of Sciences of the United States of America 114 (34), p. 8980-8985.

Zhang, W.; Fraiture, M.; Kolb, D.; Löffelhardt, B.; Desaki, Y.; Boutrot, F. F. G. et al. (2013a): Ara bidopsis receptor-like protein30 and receptor-like kinase suppressor of BIR1-1/EVERSHED mediate innate immunity to necrotrophic fungi. In: The Plant cell 25 (10), p. 4227-4241.

Zhang, X.; Han, X.; Shi, R.; Yang, G.; Qi, L.; Wang, R.; Li, G. (2013b): Arabidopsis cysteine-rich receptor-like kinase 45 positively regulates disease resistance to Pseudomonas syringae. In: Plant physiology and biochemistry: PPB 73, p. 383-391.

Zhou, J.; Wang, P.; Claus, L. A. N.; Savatin, D. V.; Xu, G.; Wu, S. et al. (2019): Proteolytic Processing of SERK3/BAK1 Regulates Plant Immunity, Development, and Cell Death. In: Plant physiology 180 (1), p. 543-558.

Zhou, J.; Wu, S.; Chen, X.; Liu, C.; Sheen, J.; Shan, L.; He, P. (2014): The Pseudomonas syringae effecttor HopF2 suppresses Arabidopsis immunity by targeting BAK1. In: The Plant journal: for cell and molecular biology 77 (2), p. 235-245.

Zhou, Q.; Zhang, Z.; Liu, T.; Gao, B.; Xiong, X. (2017): Identification and Map-Based Cloning of the Light-Induced Lesion Mimic Mutant 1 (LIL1) Gene in Rice. In: Frontiers in plant science 8, p. 2122.

Zhou, Z.; Wu, Y.; Yang, Y.; Du, M.; Zhang, X.; Guo, Y. et al. (2015): An Arabidopsis Plasma Membrane Proton ATPase Modulates JA Signaling and Is Exploited by the Pseudomonas syringae Effector Protein AvrB for Stomatal Invasion. In: The Plant cell 27 (7), p. 2032-2041.

Zipfel, C.; Kunze, G.; Chinchilla, D.; Caniard, A.; Jones, J. D. G.; Boller, T.; Felix, G. (2006): Perception of the bacterial PAMP EF-Tu by the receptor EFR restricts Agrobacterium-mediated transformation. In: Cell 125 (4), p. 749-760.

Zipfel, C.; Robatzek, S.; Navarro, L.; Oakeley, E. J.; Jones, J. D. G.; Felix, G.; Boller, T. (2004): Bacterial disease resistance in Arabidopsis through flagellin perception. In: Nature 428 (6984), p. 764-767. 
Zulawski, M.; Schulze, G.; Braginets, R.; Hartmann, S.; Schulze, W. X. (2014): The Arabidopsis Kinome: phylogeny and evolutionary insights into functional diversification. In: $B M C$ genomics 15 , p. 548 . 


\section{Supplemental material}

A

PDLP5

GNK2

CRK8

CRK6

CRK15

CRK10

V CRK5

CRK4

CRK23

CRK19

CRK13

CRK11

CRK34

CRK33

CRK14

IV CRK18

CRK18

CRK17

CRK32

CRK31

CRK2 4

III CRK3

CRK37

CRK28

I CRK41

CRK26

CRK27

CRK46

CRK2

CRK

CRK1
CRK42

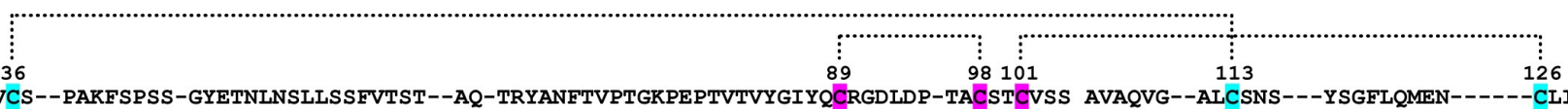
TDNYIYAVCS--PAKFSPSS-GYETNLNSLLSSFVTST--AQ-TRYANFTVPTGRPEPTVTVYGIYQCRGDLDP-TACSTCVSS AVAQVG--ALCSNS---YSGFLQMEN------CLIRYDN NPFYLNHDCPN-RTTYSSNS-TYSTNLKTLISSFASRN-ASYSTGFON--IRAGOT--PDRVTGLFICRGDLSP-EVCSNCVAF SVNESL--TRCPNO---REAVFYYEE------CIIRYSE DPFYLNHYCPN-TTTYSSNS-TYSTNTRTLSSLSSRN-ASYSTGFON--ATAGKA-- PDRVTGLFICRGDVSP-EVCRNCVAF SVNOTL--NLCPKV---REAVFYYEO------CILRYSH DPRFLAYYCPN-ATTYSSNS-TYLTNLKTLLSSLSSRN-ASYSTGFQN--ATVGAA--IDRVTGLFLCRGDVSP-EVCRNCVTF AVINTFF--SRCPNQ---REAVFYYEE------CILRYS DPRFLAYYCPN-ATYSSNS-TYLTNLKTLLSSLSSRN-ASYSTGFQN--ATVGQA--LDRVTGLFICRGDVSP-EVCRNGVTF AVNNTF--SRCPNQ---REAVFYYEE------CILRYS DPTYVYHTCQN-TANYTSNS-TYNNNLKTLLASLSSRN-ASYSTGFQN--ATVGQA--PDRVTGLFNCRGDVST-EVCRRCVSF AVNDTL--TRCPNQ---KEATLYYDE-----CVLRYSN DPTYVGHVCTN---RISPNS-IYTSTI DPTYVGHCTN---RISRNS-IYFSNLQTLLTSLSSNN-AYFSLGSHS--LTKGQN--SDMFFLYLCKGDLSP-ESCRECVIF AAKDTR--SRCPGG---KEFLIQYDE------CMLGYSD NFIHLNHSCPSSILTYSRNS-TYFTNLKTLLSSLSSRN-ASYSTGFQT--ATAGQA--PDRVTGLFLCRGDVSQ-EVCRNCVAF SVKETL--YWCPYN---KEVVLYYDE------CMLRYS DPTYVYHVCPS-WATFPRSS-TYMTNLITLLSTLSSP-SASYSTGFQN--ATAGRH--PDRVTGLFNCRGDVSP-EVCRRCVSF AVNETS--TRCPIE---KEVTLYYDQ------CTLRYS NPFYLYANCSI-TYYSSNS-TYSTNLKILLSSLSSRN-ASYSTGFQN--ATAGQA--PDNVL NTFYLYHNCSV-TTTFSSNS-TYSTNLKTLLSSLSSLNASSYSTGFQT--ATAGQA--PDRVTGLFLCRVVSS-EVCRSCVTF AVNETL--TRCPKD---KEGVFYYEQ-------CLLRYS
DPTYLYHICPN-TTTYSRNS-SYLTNLRTVLSSLSSPN-AAYASLFDN--AAAGEENDSNRVYGVFLCRGDVSA-EICRDCVAF AANETI---QRCPRE---KVAVIWYDE------CMVRYSN AS-VSAQTCIENRKYFTPNG-TYDSNRRLILSSLPNNT-ASQ-DGFYY--GSIGEE--QDRVYALGMCIPRSTP-SDCFFCIKG AAGWLI--QDCVNQ---TDAYYWALD---PTLCLVRYSI AS-VSAQTCIENRKYFTPNG-TYDSNRRLILSSLPNNT-ASR-DGFYY--GSIGEE--QDRVYALGMCIPKSTP-SDCSNCIKG AAGWLI--QDCVNQ---TDAYYWALD---PTLCLVRYSN AS-VSAQTCTTDKGTFRPNG-TYDVNRRLILSSLPSNV-TDQ-DGLYYN-GSIGQQ--PNRVYAIGMCIPGSTS-EDCSDCIKK ESEFFL--KNCPNQ---TEAYSWPGE---PTLCYVRYSN DS-VTAOE-ICFSGFFKPNS-TYDLNRROILSTLSSSNV-TSH-NGFFN--SKFGQA--PNRVFINGMCIPGTKP-ETCSDCIKG ASDKIS--ESCPNK---TDAYTWP-------DCCMVRYSN IGAISSOOCN-ETGYFEPWK-TYDTNRROILTSLASKV-VDH-YGFYN--SSIGKV--PDFVHVMGMCIDGTEP-TVCSDCIKV AADOLO--ENCPNO---TEAYTWTPH---KTLCFARYSN V---SAOECG-KTGFFVPOS-RYETNRGILISSIPSNV-SAR-GGEYN--SSIGOG--PDRVYALGMCTEGAEP-DVCSDCTFY ASNLTL--DTCLNO---TEGLAWPEK---RILCMVRYSN VSALOTLPCI-NTTYFIPNS-TYDTNRRVIISLIPSNV-TSH-FGEFN--GSIGOA--PNRVYAVGMCLPGTEE-ESCIGCISS ASNTLI--ETCLTE---ENALIWIAN---RTICMTRYSD ISVS-AOTCDNTTGTFTPNS-PYDKNRRLIISTIASNV-TAQ-EGYFT--GSIGIA--PDQVEFATGMCAPGSER-DVCSICIRS TSESLL--OSCLDQ---ADAFFWSGE---ETLCLVRYAN ISVAOOTCD

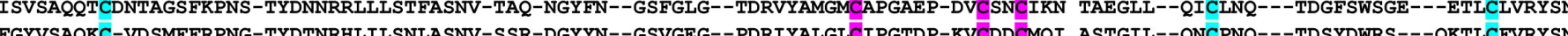
作 FGFVSAQKC-GESVFRPNG-NYDTNRRLVSSTLASNV-SSQNGR IIVSALCSER S-ISSAQKC-GKTGLFKPND-KYDINRHLLLSSLASNV-SAR-GGFYN--ASIGQG--PDRLYALG_LIQGSLPLLCSACIDS AFIRVI--KKCHNQ---TEALDWSSFN-EEYPCMIRYS C-AVAAPTCIQRSDFFKANG-PYDINLRAMLSSIPSRV-KDN-EGFYK--TPFKPG--PNIAHGLGMCSRGTTT-QDCSDCITS VSHTLL--HTCPNQ---AEAIDWSSG---DSLCLVRYS EIVN-AVGCT--GSFFNGNS-SYAQNRRDLFSTLPNKV-VTN-GGFYN--SSLGKSP--NIVHAVALCGRGYEQ-QACIRCVDS AIQGILTTTSCLNR---VDSFTWDKDEEDNVSCLVSTSN HVVN-AVKCF--GNSFNGNSSTFAQNRQKLFPTLADKV-IIN-DGFYN--ASLGODP--DKVYALVSCARGYDQ-DACYNCVQS LTQNTL--TDCRSR---RDSFIWGGN--DDVTCLVRSS HGVKAGFICV--GSSFPTNS-SYQKNRDSLFSTLSDDKV-TTN-GGFYN--ASLD------GVHVVGLCRRDYDR-QGCINCVEE SIRQIK--TSCSNR---VQSFHCNSDDRERVSCLVRTTD KYVHAGFICY--GDFFNVN---YGVSRTYLFSSLPSNV-VSN-GGFYN--ASFGRDSKNNRVHVVALCRRGYEK-OACKTCLEH VIEDTK--SKCPRO---KESFSWVTDEFDDVSCSLRYTM NGVHTTFVCG--DEDFSPNT-SYVENLESLLPSLASNV-IRE-RGFYN--VSLD------GVYALALCRKHYEV-OACRRCVDR ASRTLL--TOCRGK---TEAYYHWDSENDANVSCLVRYSN YEFPPGFNCVASGGNFTANS-SFAGNLNGLVSSLSSLT--SKPYGFYN--LSSGDSS-GERAYAIGLCRREVKR-DDCISCIQI AARNLI--EQCPLT---NQAVVWYTH-------CMFRYSN YEFDPDFNCVDR-GNFTANS-TFAGNLNRLVSSLSSLK--SQAYGFYN--LSSGDSS-GERAYAIGLCRREVKR-DDCVSCIQT AARNLT--KQCPLT---KQAVVWYTH-------CMFRYSN WEFPSNPLCLSQQSNFAKSS-QFSKNLDSLVSSIPSLK--SNTYNFYS--LSVGSISDQERVEAIGICNRVVNR-VDCLNCIAQ AAVNLT-TMYCPQH---RGAYVRATK------CMFRYS QPVPLNQICSNVTGNFTVNT-PYAVNLDRLISSLSSLR--RNVNGFYN--ISVGDSD--EKVNSISQCRGDVKL-EVCINCIAM AGKRLV--TLCPVQ---KEAIIWYDK------CTFRYS QNVTVHTICYYDGGNFTSNT-SYSLNLNRLISSLPDLT--PTINGFYN--ISIN-----GEVNAIALCRGDVKPNQDCISCITT AAKQLV--ESCPNI---IEANIWLEK-------CMFRYT RIDVLGYICNNGTVSN--EE-AYRRSYQINLDAIRGDM--RH-VKFGTH-E-HGDP--PERMYVLSQCVSDLSS-DECSLCWSR ATDLIS--QCFPA----TGGWFHLDG------CFVRADN RARAVKVTCSPLIEHN--ET-AYVPNFVATMEKISTQV--QT-SGFGVA-L-TGTG--PDANYGLAQCYGDLPL-NDCVLCYAE ARTMLP--QCYPQ-----NGGRIFLDG-------CFMRAEN RGDTVAQICNNRTTTPQQRS-LFVTNFLAAMDAVSPLV--EA-KGYGQV-V-NGTG--NLTVYAYGECIKDLDK-KDCDLCFAQ IKAKVP--RCLPFQKGTRGGQVFSDG-------CYIRYDD SSSESLLNCQPIDHHLV-NP-SRLIGFLRAMSSVNDFI--TN-DKLWVV-SSITDV--SPPIYVFLQCREDLSV-SDCRHCFNE SRLELE--RKCSG----SGGRIHSDR------CFLRFDD RTTVSGLFCGGRSKSSA-DP-NYIPTFVEDMHSLSLKL--TT-RRF--A-TESLNS--TTSIYALIQCHDDLSP-SDCQLCYAI ARTRIP--RCLPS----SSARIFLDG------CFLRYET 
GELIMR----NPNNISSIQNQRDQFIDLVQSNMNQAANEAA-------N-SSRKF-STIKTELTSIQTLYYGLVQ

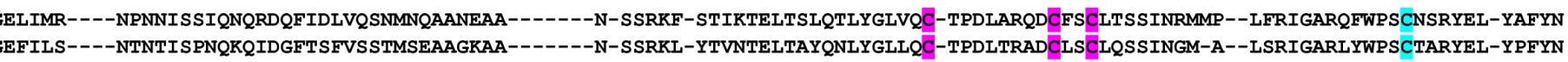
GEFILR----NPNHISPIQNQINQFTNLVLSNMNQIAIEAA-------D-NPRKF-STIKTELTALQTFYGLVO-TPDLTRADCLSCIQSSINGM-A--LSRIGARLYWPSCTARYEL-YPFYN GAWIRM-----NGNISIDQNOMNRFKDFVSSTMNQAAVKAA-------S-SPRKF-YTVKATWTALQTLYGLVOC-TPDLSRQNCMNCLTSSINRM-P--FSRIGARQFWPSCNSRYEL-YDFYN

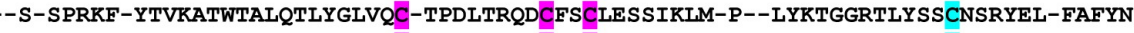
GVILV----NTRN--VTSNQLDLLSDLVLPTLNQAATVAL------N-SSKKF-GTRKNNFTALQSFYGLVQC-TPDLTRQDCSRCLQLVINQI-P--TDRIGARIINPSCTSRYEI-YAFYT TIITWN----TQKVTA---DQSDRFNDAVLSLMKKSAEEAA-------NSTSKKF-AVKKSDFSSSQSLYASVQC-IPDLTSEDCVMCLQQSIKEL-Y--FNKVGGRFLVPSCNSRYEV-YPFYK SAILLN----GANISSSNQNQVDEFRDLVSSTLNLAAVEAA-------N-SSKKF-YTRKV--ITPQPLYLLVQC-TPDLTRQDCLRCLQKSIKGM-S--LYRIGGRFFYPSCNSRYEN-YSFYN GIILAN----SQNMTSN---EQARFKDLVLTTMNQATIAAA-------N-SSKRF-DARSANFTTLHSLYTLVQC-THDLTRQDCISC IQQIIINQL-P--TEKIGGQFIVPSCSSRFEL-CLFYN GVFMQN----ARNPISV---RQDRFRDLVLNPMNLAAIEAA-------R-SIKRF-AVTRFDLNALQSLYGMVQC-TPDLTEQDCIDCLQQSINQV-T--YDRIGGRTFLPSCTSRYDN-YEFYN GMFMQS----ARNPLSV---KQDQFRDLVLTPMNLAAVEAA-------R-SFKKW-AVRKIDLNASQSLYGMVRC-TPDLREQDCLDCLKIGINQV-T--YDKIGGRILLLPSCASRYDN-YAFYN GVFLTN----KQNITE---NQVSRFNESLPALIIDVAVKAA-------L-SSRKF-ATEKANFTVFQTIYSLVQC-TPDLTNQDCESCIRQVINYLPRCCDRSVGGRVIAPSCSFRYEL-YPFYN IEPQYLV--LNTATIASDLTDFKNIWEDLTSRTITAASAARS---TPSSSDN-HY-RVDFANLTKFQNIYALMQC-TPDISSDECWNCLLQRGVLEYQSCCGNNTGGYVMRPICFFRWQL-FTFSK IEPQYLV--LNTATIASNLTEFKTIWEDLT SRTITAASAARS---TPSSSDN-HY-RVDFANLTKFQNIYALMQC-TPDISSDECNNCCLQRGVLEYQSCCGNNTGGYVMRPICFFRWQL-FTFSK LNPRNWL--TNTGDLDSNLTEFTKIWEGLMGRMISAASTARS---TPSSSDN-HY-SADSAVLTPLLNIYYALMOC-TPDLSSGDCENCLRQSAIDYQSCCSOKRGGVVMRPSCFLRWDL-YTYSN SETLYHT--GDIEDTGTNLTVFDRIWEELMLRTITAASLSSS---NGSSFGQKYF-AAEVASLTTFQTMYAMMQC-TPDVSSKDCEFCLIKTSVGDYESCCRGKQGGAVIRPSCFVRWDL-YPYAG LHPLYME--HSNVDIKSNLTYLNTIWEALTDRLMSDASSDYN---ASLS-SRRYY-AANVTNLTNFQNIYALMLC-TPDLEKGACHNCLLEKAVSEYGNL--RMQRGIVAWPSCCFRWDL-YPFIG AEPHFYI--HNVDDIT SNLTEFDQVWEELARRMIASTTS-------PSS-KRKYY-AADVAALTAFQIIYALMQC-TPDLSLEDCHICLRQSVGDYETCCNGKQGGIVYRASCVFRWEL-FPFSE LEPHREF--LSIHGYKTNETEFNTVWSRLTQRMVQEASSSTD---ATWS-GAKYY-TADVAALPDSOTLYAMMQC-TPDLSPAECNLCLTESVVNYQSCCLGRQGGS IVRLSCAFRAEL-YPFGG LGAIFNTGELNTN-----QTVFDIEWNNLTSSSMIAGITSSSS----GGNNSSKYY-SDDIALVPDFKNISALMQC-TPDVSSEDCNTCLLRQNVVDYDNCCRGHQGGVMSRPNCFFRWEV-YPFSG SNDFFNVNEIRKE----DQKEFDSVFDELMFRTIQGASSSVRNNSNSLSLSGKYY-AKDVAPEPVYGNISVVMQC-TPDVSSKDCNLCLERSSLDFYKKWYNGKRGTIIILRPSCFFRWEL-YTFFG TMV---IGDLNSGLFQGDLAAYTRTWEEFMNSMI TRVGR--TRY--LADISPR------IGS-----ARIYALMQC-IRGISSMECETCIRDNVRMYQSCCNGF IGGT IRKPVCFFRWDG-SEYLG THA---V--YNTMRFQGNLTAYTRTWDAFMNFMFTRVGQ--TRY--IADI SPR------INQEPLSPDLIYALMOC-IPGISSEDCETCLGKCVDDYOSCCNGFIGGVVNKRPVCYFRWDG-YKYYG VS-----IGYNVGNLSTNLTDFDRLWERLIAHMVTKASSASIKY--LSFDNSRFY-AADETNLTNSOMVYALMOC-TPDVSPSNCNTCLKOSVDDYVGCCHGKOGGYVYRPSCIFRWDL-YPFNG FFRNYNA-----TDFQVNLTEFYQKWEALMLGVIADAISSPN--------- PKFY--GAGTGKIGIQTVYAFVLC-SKDISPWNCSRCLRGNVDNYKLSCSGKPRGHSFSPSCYMRWDL-YQFYG IWAEYIEYKYNTSFGQTNLTEFKSTWQAL-MDRVINKVDGSL----------YANS--IQELGSFPFRSIYAIAQC-NKDLTKLNCERCLQHLRIDNRSCCRGIQVGY IARTSCFMRWDL-QPFLG SVRYQSP--NSIEP-SKNMTLFEQEWNAMANRTVESATEAET-------SSVLKYY-SAEKAEFTEFPNVYMLMQC-TPDITSQDCKTCLLGECVTLFKEQVWGRQGGEVYRPSCFFRWDL-YAFHG PVVWPSP--DTIES-SKNITLFKQQWEEMVNRTLEAATKAEG------SSVLKYY-KAEKAGFTEFPDVYMLMQC-TPDLSSRDCKQCL GDCVVMYFRKDYMGRKGGMASLPSCYFRWDL-YSFHN ATNDPSP--VAIDTFAKNMTLFRQEWEAMVDRTLEAVTIDNS------TTVLRYY-GALKSEFSEFPNVYMMMQC-TPDINSGACKRCLQASVTYFRDQNWGRQGGGICRPSCVFRWEF-YPFYG NTINPNP--NSIDSKFNNMAMF SQEWIAMVNRTLEAASTAEN------SSVLKYY-SATRTEFTQI SDVYALMQC-VPDLSPGNCKRCLLRECVNDFQKQFWGRQGGGVSRPSCYFRWDL-YPYYR IGNVPHS---SLDP-SSNLTRISQEFAARANRTVEVASTADE------SSVLKYY-GVSSAEFTDTPEVMMLMQC -TPDLSSSDCNNHCLLRENVRYNQEHNWDRVGGTVARPSCYFRWDD-YRFAG TLSFQAG-----KNISANRDEFDRLQIELIDRLKGIAAAGGP--------NRKYA-QGSGSGVAGYPQFYGSAHC-TPDLSEQDCNDCLLVFGFEKIPGCCAGQVGLRWFFPSCSYRFET-WRFYE TKAFIAG-----EEISANRDDFERIQRGLIDRLKGIAAAGGP-------NRKYA-QGNGSASAGYRRFYGTVQC-TPDLSEQDCNDCLIVFGFENIPSCCDAEIGLRWFSPSCNFRFET-WRFYE TKAFIAG-----EEISANRDDFERLQRGLLDRLKGIAAAGGP--------NRKYA-QGNGSASAGYRRFYGTVQC-TPDLSEQDCNDCLVFGFENIPSCCDAEIGLRWFSPSCNFRFET-WRFYE VLEAPNP-----SNATGDRNEFIRLQSELLNRLRSMAASGGS--------KRKYA-QGTDPGSPYTTFFGAVQC-TPDLSEKDCNDCLSYGFSNA---TRGRVGIRWFCPSCNFQIESDLRFFL

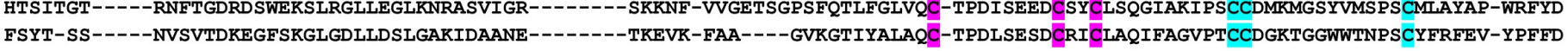
DTKICAS----D---K---EKSAEFKGLVKEVTKSIVEAAPY--------SRGFSVAKMGI---RDLTVYGLGVC-WRTLNDELCKLCLLADGALS-VTSCLPSKEGFALNAGCYLRYSN-YTFYN DSIVCGN-------TT---RKNKTFGDAVRQGLRNAVTEASG--------TGGYARASARAGESESESAFVLANC-WRTISPDSCKQCLLENASASVVKGCLPWSEGRALHTGCFLRYSD-QDFLN DRTVCAP----KEITG---VNRTVFRDNAAELVKNMSVEAVR---------NGGFYAGFVDR---HNVTVHGLAQC-WETLNRSGCVECLISKASVR-IGSCLVNEEGRVLSAGCYMRFST-QKFYN FDKANCE----E--TG---TGFGEFWRFLDEALVNVTLKAVK--------NGGFGAASVIK----TEAVYALAQC-WQTLDENTCRECLVNARSS-LRACD-GHEARAFFTGCYLKYST-HKFFD SDSFSCS----N--DT---VLDPRFGFQVSE---TAARVAVR--------KGGFGVAG-------ENGVHALAQC-WESLGKEDCRVCLLEKAVKE-VKRCVSRREGRAMNTGCYLRYSD-HKFYN 
Figure S1: Cysteine residues in DUF26 domains are highly conserved.

Alignment of DUF26-A (A) and DUF26-B (B) domains of all Arabidopsis CRKs with the DUF26 domain(s) of Ginkgo biloba GNK2 and Arabidopsis PDLP5. CRK9 and CRK35 that are not considered as CRKs anymore and the truncated CRKs CRK43-CRK45 lacking the ectodomain were excluded from the alignment. Cysteine residues belonging to the highly conserved $\mathrm{C}-\mathrm{X}_{8}-\mathrm{C}-\mathrm{X}_{2}-\mathrm{C}$ motif are highlighted in pink, additional cysteine-residues are highlighted in cyan. Numbers above the cysteine residues refer to amino acid positions in PDLP5 (Vaattovaara et al., 2019). Yellow highlighted residues were identified as essential for mannose binding in GNK2 (Miyakawa et al., 2014). Dotted lines indicate disulfide bridges identified in GNK2 and PDLP5 (Miyakawa et al., 2009; Vaattovaara et al., 2019). Roman numerals on the left refer to CRK subgroups according to Bourdais et al., 2015. 


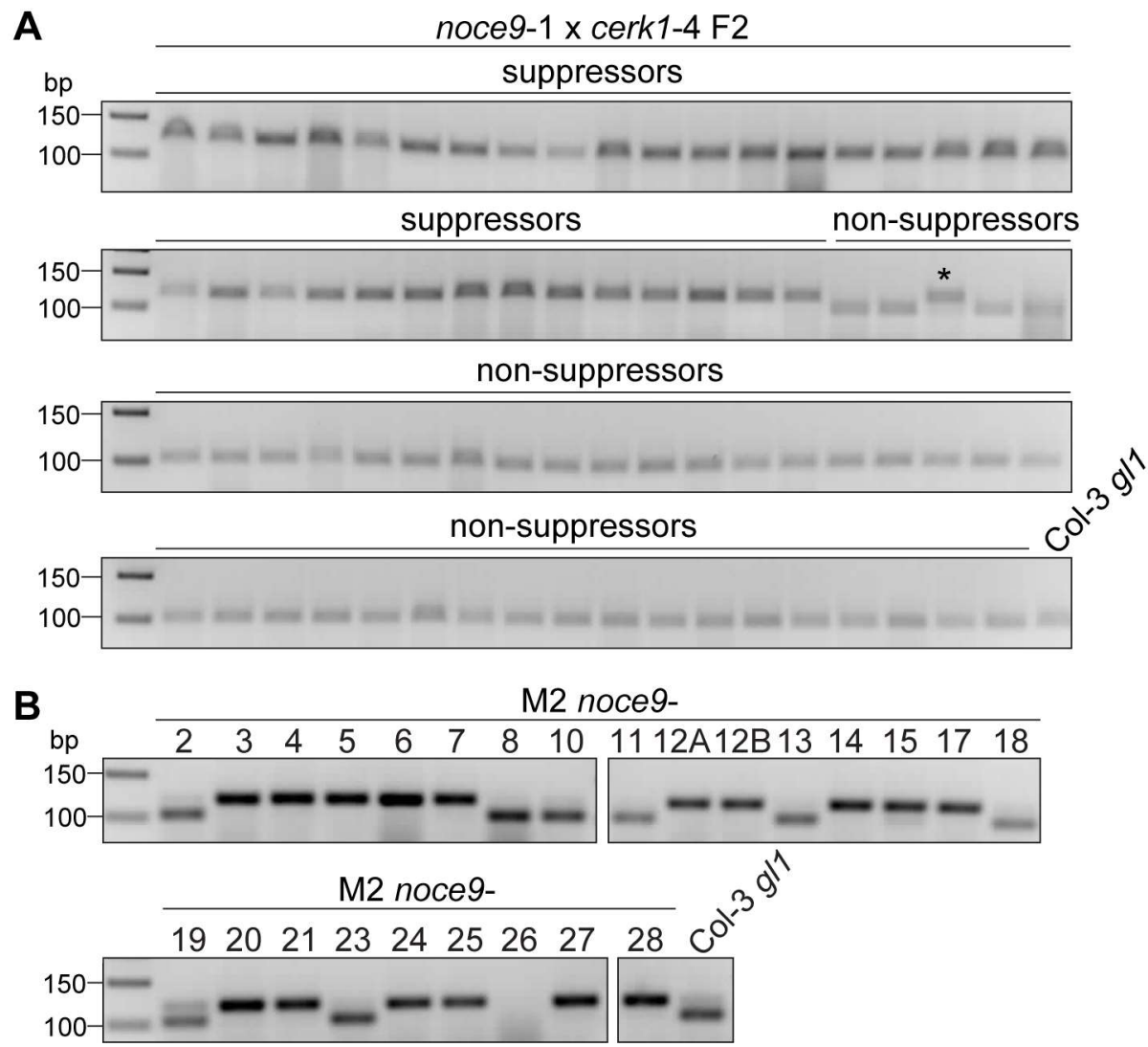

\section{Figure S2: Genotyping of $c r k 7-4$ in different plant lines.}

The $c r k 7-4$ mutation was genotyped by PCR using oCT3 + oCT4 and restriction digest of the PCR product with PdiI. PdiI cuts the $136 \mathrm{bp}$ PCR fragment only in CRK7 (104 bp $+32 \mathrm{bp}$ ) but not in crk7-4. Col-3 gll was included as control A: Genotyping of crk7-4 in 33 noce9-1 x cerk1-4 F2 plants with suppressor and 42 noce9-1 x cerk1-4 F2 plants with non-suppressor phenotype. All plants with suppressor phenotype are homozygous for $c r k 7-4$, whereas $41 / 42$ non-suppressor plants are homozygous for WT CRK7. The asterisk marks a plant scored as non-suppressor but heterozygous for crk7-4. B: Genotyping of noce9 M2 plant lines, 16 out of 25 lines carry the $c r k 7-4$ mutation. 
A

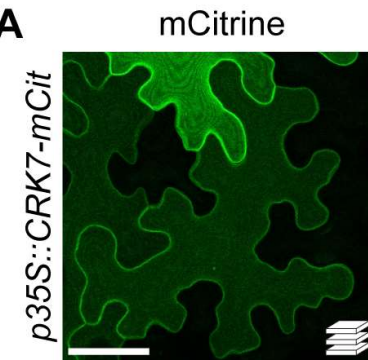

mKate2-SYP122

overlay
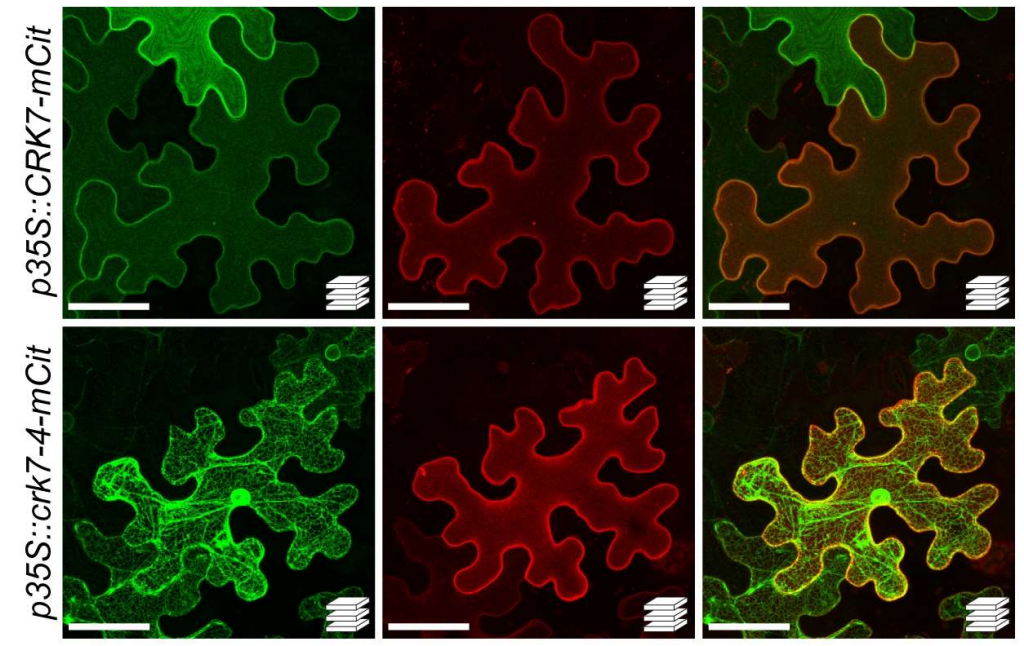

B
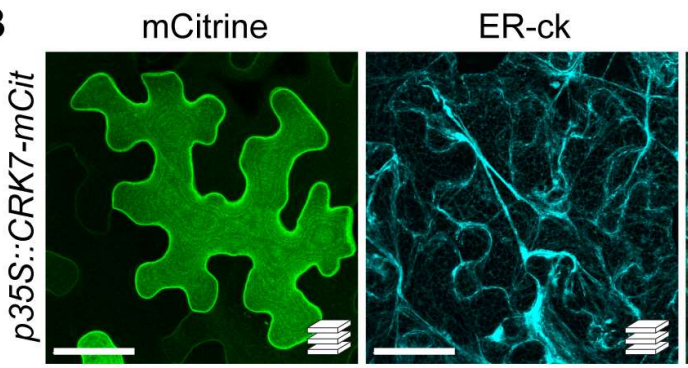

overlay
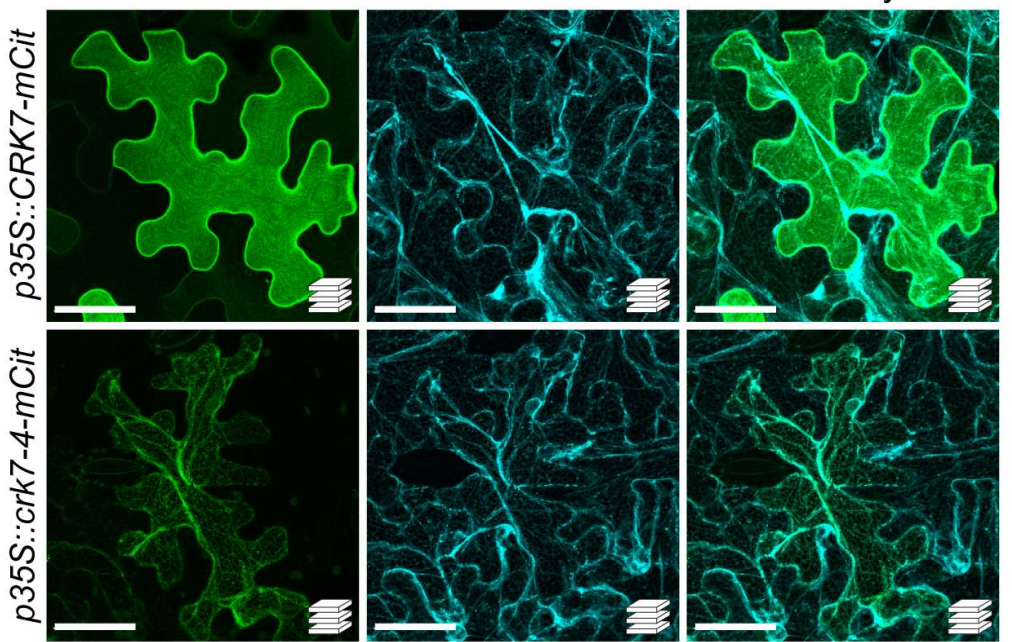

C

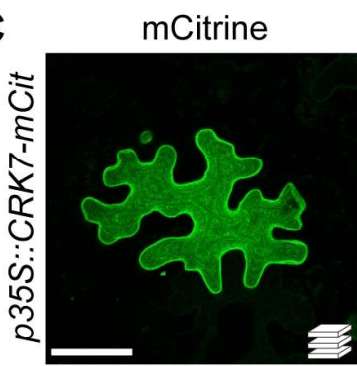

mKate2-N7

overlay
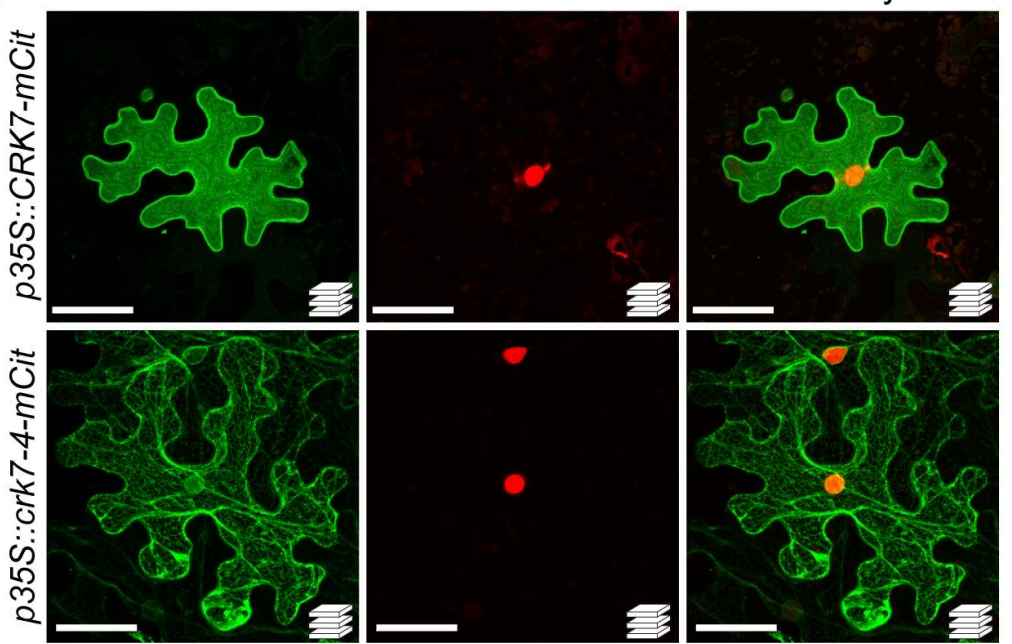

Figure S3: CRK7-mCit and crk7-4-mCit co-infiltrated with cellular markers.

A. tumefaciens cultures transformed with $p 35 S: \because C R K 7-m C i t r i n e ~(m C i t)$ or $p 35 S:: c r k 7-4-m C i t$ were mixed with cultures transformed with $p U B Q 10:: m$ Kate2-SYP122, p35S::ER-ck or $p U B Q 10::$ mKate2-N7 prior to infiltration into $N$. benthamiana. Confocal microscopy (Leica SP8) was performed 2-3 dpi. Images are representative maximum projections. Scale bar $=50 \mu \mathrm{M}$, Z-stack size $19 \mu \mathrm{M}$. Green $=$ mCitrine. A: Coinfiltration with mKate2-SYP122 (red; E. Petutschnig, unpublished). B: Co-infiltration with ER-ck (turquoise; Nelson et al., 2007). C: Co-infiltration with mKate2-N7 (red, H. Ghareeb, unpublished). 
A

\begin{tabular}{|c|c|c|c|c|c|c|}
\hline \multirow{2}{*}{ No } & \multirow{2}{*}{ Sequence } & \multirow{2}{*}{ Charge } & \multicolumn{2}{|c|}{ Modifications } & \multirow{2}{*}{\multicolumn{2}{|c|}{ Localization in protein }} \\
\hline & & & & & & \\
\hline 1 & KTYGTTPALDEDDKTTIESLQLDYR & 3-4 & 1 & & Juxtamem & brane domain \\
\hline 2 & TYGTTPALDEDDKTTIESLQLDYR & 3 & 1 & & & \\
\hline 3 & GGFGDVYKGTFSNGTEVAVKR & 3 & 1 & & & $1+11$ \\
\hline 4 & IFGMDQTQQNTSR & 2 & 1 & $0-1$ & & AL \\
\hline 5 & IFGMDQTQQNTSRIVGTYGYMSPEYAMR & 3 & $1-2$ & $0-3$ & & VIII, AL, P+1 \\
\hline 6 & IVGTYGYMSPEYAMR & 2 & 1 & $0-2$ & & VIII, P+1 \\
\hline 7 & SDVYSFGVLVLEIISGRKNNSFIETDDAQDLVTHAWR & 6 & 3 & & Kinase & $\mathrm{IX}+\mathrm{X}$ \\
\hline 8 & NGTALDLVDPFIADSCR & 2 & 1 & 1 & & $\mathrm{x}$ \\
\hline 9 & NGTALDLVDPFIADSCRK & $2-3$ & 1 & 1 & & $\mathrm{x}$ \\
\hline 10 & NGTALDLVDPFIADSCRKSEVVR & 3 & 1 & 0 & & $\mathrm{x}$ \\
\hline 11 . & CTHIGLLCVQEDPVKRPAMSTISVMLTSNTMALPAPQQPGFFVR & 4 & 1 & $0-1 \quad 2$ & & $\mathrm{XI}$ \\
\hline 12 & SRPGTNRLDSDQSTTNK & $2-3$ & $1-2$ & & & \\
\hline 13 & SRPGTNRLDSDQSTTNKSVTVSIDDK & 3-4 & $1-2$ & & & \\
\hline 14 & LDSDQSTTNKSVTVSIDDK & 3 & 1 & & C-terminus & \\
\hline 15 & LDSDQSTTNKSVTVSIDDKSMSDLDPR & 4 & 1 & & & \\
\hline 16 & SVTVSIDDKSMSDLDPR & $2-3$ & 1 & 1 & & \\
\hline
\end{tabular}

B

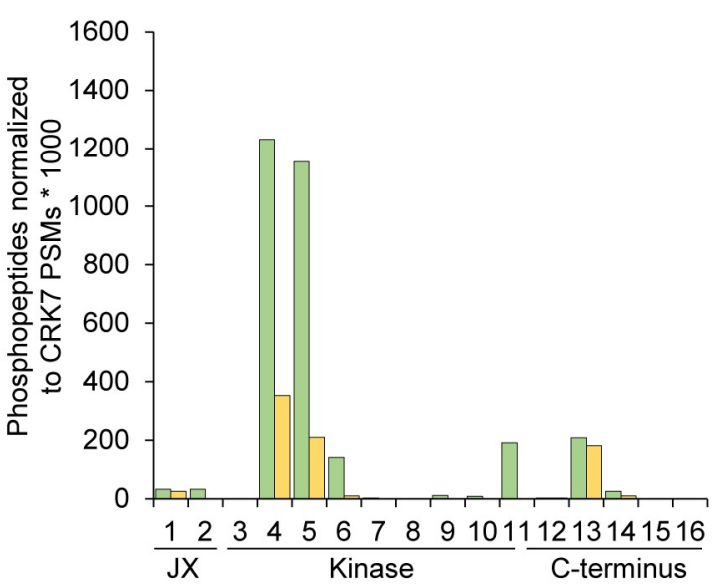

p35S::CRK7-mCit

p35S::crk7-4-mCit

Figure S4: CRK7 shows higher levels of phosphorylation in the activation and P+1 loop than CRK74.

p35S::CRK7-mCitrine ( $m$ Cit) and $p 35 S: \because c r k 7-4-m C i t$ were transiently expressed in $N$. benthamiana. Infiltrated leaf areas were harvested 2 dpi and proteins were extracted. An overnight $\alpha$-GFP pull-down was performed, followed by an on-bead tryptic digestion and a C18-purification. The samples were used for liquid chromatography mass spectrometry (LC-MS) with the Orbitrap Q Exactive HF mass analyzer. A: Sequences and localization of the analyzed phosphopeptides are shown. 20 phosphopeptide ions were chosen for targeted selected ion monitoring (tSIM) analysis. Peptide ions with identical sequence but different charges or modifications were summarized. Numbers of modifications are displayed; $\mathrm{P}=$ phosphorylation, $\mathrm{O}=$ Oxidation, $\mathrm{C}=\mathrm{C}$ arbamidomethylation. Roman numerals refer to kinase subdomains. $\mathrm{AL}=$ activation loop; $\mathrm{P}+1=\mathrm{P}+1$ loop. $\mathbf{B}$ : Data show the number of identified peptide spectrum matches (PSMs) normalized to total CRK7 PSMs *1000. Numbers 1-16 refer to the phosphopeptides in A. JX= Juxtamembrane domain. 


\begin{tabular}{|c|c|c|c|c|c|c|c|c|c|c|c|c|c|c|c|c|c|c|c|}
\hline \multicolumn{2}{|c|}{ A } & \multicolumn{4}{|c|}{$\begin{array}{c}\text { Juxtamembrane } \\
\text { domain }\end{array}$} & \multicolumn{7}{|c|}{ Kinase domain } & \multicolumn{7}{|c|}{ C-Terminus } \\
\hline Amir & o acid & $\mathrm{T}$ & $\mathrm{T}$ & $\mathrm{T}$ & $S$ & $T$ & $\mathrm{~T}$ & $S$ & $\mathrm{~T}$ & $S$ & $S$ & $S$ & $\mathrm{~T}$ & $S$ & $S$ & $S$ & $S$ & $\mathrm{~T}$ & $S$ \\
\hline \multicolumn{2}{|c|}{ Position in protein } & 307 & 308 & 317 & 321 & 491 & 495 & 496 & 501 & 506 & 573 & 577 & 611 & 626 & 635 & 638 & 643 & 645 & 652 \\
\hline \multirow{2}{*}{ p35S::CRK7-mCit } & iMS & & & & & & $\bullet$ & $\bullet$ & & $\bullet$ & $\bullet$ & & & & $\bullet$ & $\bullet$ & $\bullet$ & & \\
\hline & $\mathrm{tSIM}$ & 0 & 15 & 15 & 0 & 195 & 304 & 442 & 0 & 139 & 9 & 9 & 0 & 0 & 3 & 35 & 68 & 0 & 0 \\
\hline \multirow{2}{*}{ p35S::crk7-4-mCit } & iMS & & & & & & & & & & & & & & $\bullet$ & & $\bullet$ & & \\
\hline & $\mathrm{tSIM}$ & 0 & 0 & 0 & 0 & 0 & 71 & 162 & 0 & 10 & 0 & 0 & 0 & 13 & 16 & 10 & 55 & 0 & 0 \\
\hline
\end{tabular}

B

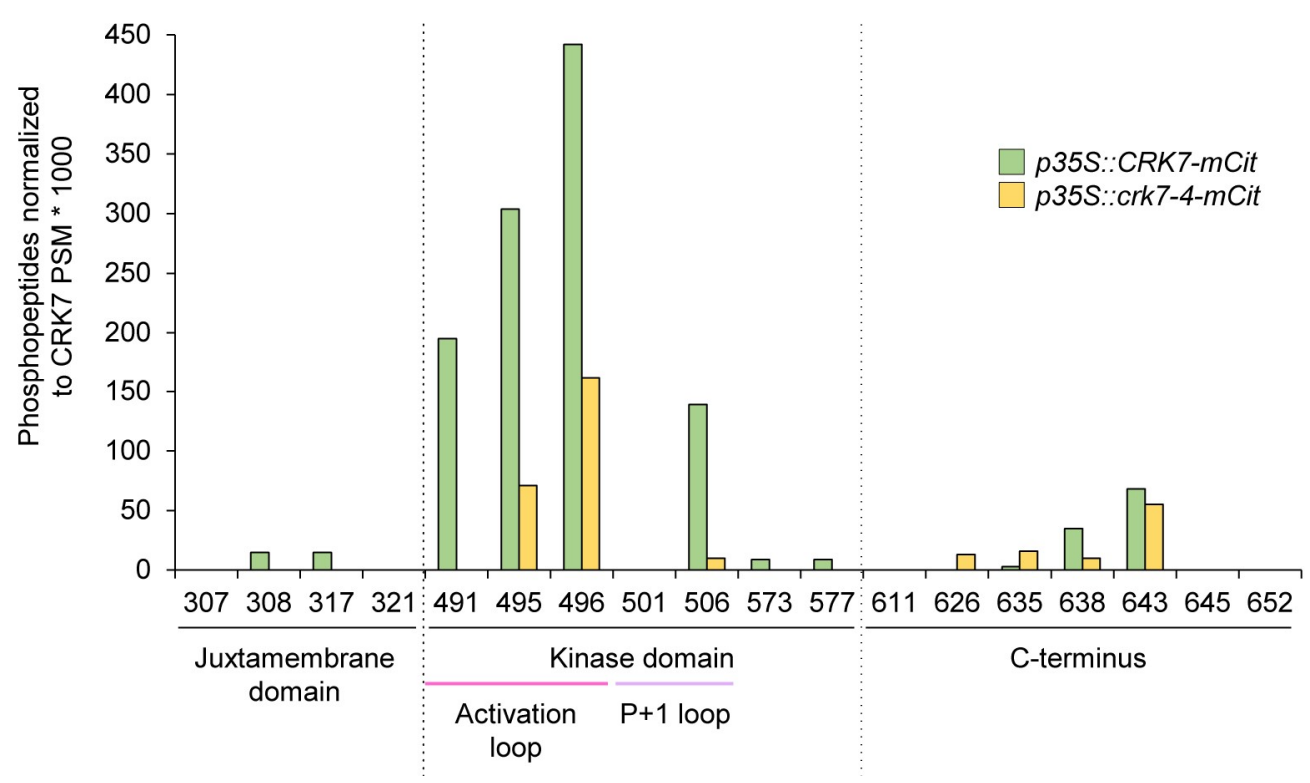

Figure S5: Phosphorylation sites identified in CRK7 and CRK7-4.

p35S::CRK7-mCitrine ( $m$ Cit) and p35S::crk7-4-mCit were transiently expressed in $N$. benthamiana. Infiltrated leaf areas were harvested $2 \mathrm{dpi}$ and proteins were extracted. An overnight $\alpha$-GFP pull-down was performed, followed by an on-bead tryptic digestion and a C18-purification. The samples were used for LC-MS with the Orbitrap Q Exactive HF mass analyzer. 20 phosphopeptides were chosen for targeted selected ion monitoring (tSIM) analysis. Phosphorylation sites were identified with the ptm $R S$ node. Numbers of identified phosphorylation sites with a probability of $\geq 95 \%$ normalized to the total CRK7 PSMs are displayed. A: Dots represent phosphorylation sites identified in the initial LC-MS run (iMS). Numbers show counts of identified phosphorylation sites normalized to total CRK7 PSMs * 1000. Amino acids highlighted in pink belong to the activation loop, amino acids highlighted in purple to the $\mathrm{P}+1$ loop. B: Phosphorylation sites identified in tSIM runs by $\operatorname{ptm} R S$ with a probability of $\geq 95 \%$ normalized to the total CRK7 PSMs * 1000 . 


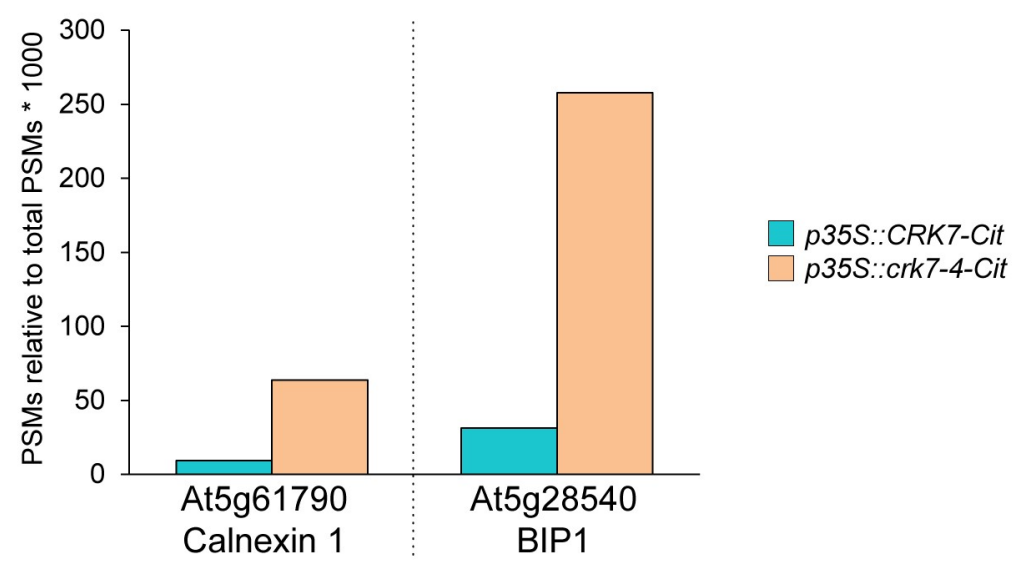

Figure S6: CRK7-4 interacts with ER chaperones in Arabidopsis.

p35S::CRK7-Citrine (Cit, turquoise) and p35S::crk7-4-Cit (orange) were stably transformed into cerk1-4 plants. Proteins were extracted from six-week old T2 plants. An overnight $\alpha$-GFP pull-down was performed, followed by an on-bead tryptic digestion and a C18-purification. The samples were used for LCMS with the Orbitrap Q Exactive HF mass analyzer. Peptide spectrum matches (PSMs) matching chaperones that potentially interact with CRK7/ CRK7-4 are shown. Only proteins with at least one protein unique peptide were considered. PSMs were normalized to total PSMs and multiplied with 1000 for better visualization. 
A

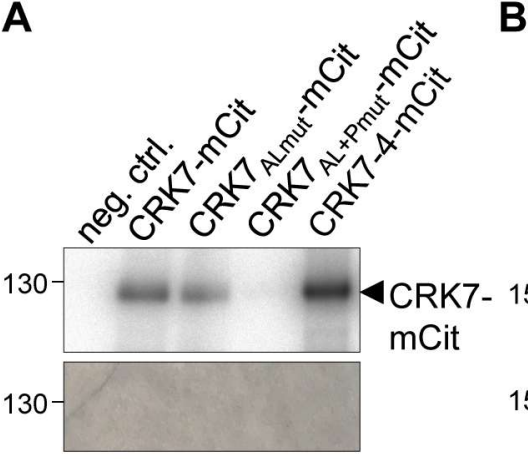

$8 \%$ gel

D

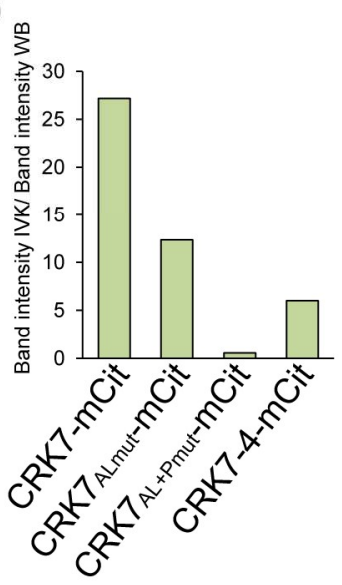

B

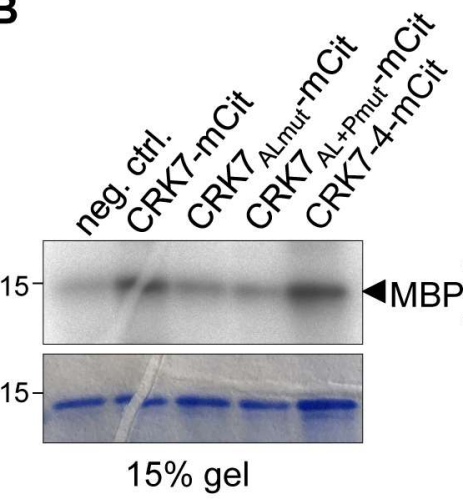

E

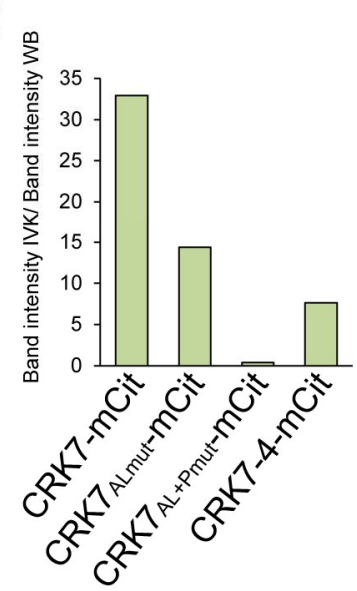

C

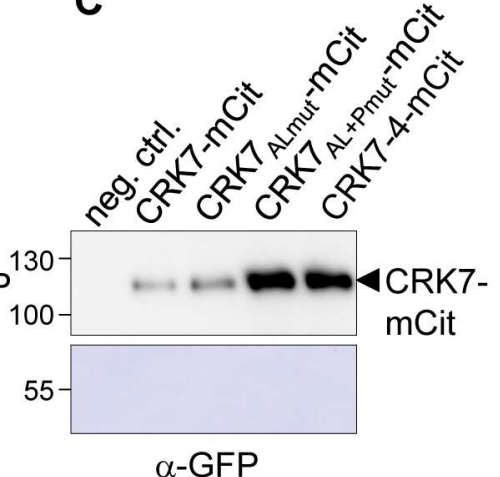

$\alpha-G F P$

\section{Figure S7: CRK7 is an active kinase whereas CRK7 $7_{\mathrm{AL}+\mathrm{Pmut}}$ is kinase dead.}

CRK7-mCit, CRK7 ALmut $^{-m C i t, ~ C R K 7 ~} 7_{\mathrm{AL}+\mathrm{Pmut}} \mathrm{mCit}$ and $\mathrm{CRK} 7-4-\mathrm{mCit}$ were transiently expressed in $\mathrm{N}$. benthamiana under the $35 S$ promotor. Expression was confirmed via CLSM and infiltrated leaf areas were harvested two dpi. Non-infiltrated leaves were used as negative control (neg. ctrl). Adjusted total protein extracts were used for an overnight GFP pull-down. CRK7 autophosphorylation (A) and phosphorylation of the artificial substrate myelin basic protein (MBP) (B) were tested. Coomassie stained gels are shown as loading controls. C: Western Blot of GFP pull-downs with the $\alpha$-GFP antibody. D $+\mathbf{E}$ : Quantification of the autoradiography band intensities of the $8 \%$ (D) and 15\% (E) gel normalized to the Western Blot band intensities. Intensities of the negative control in the autoradiograms were subtracted from the respective samples prior to normalization. 


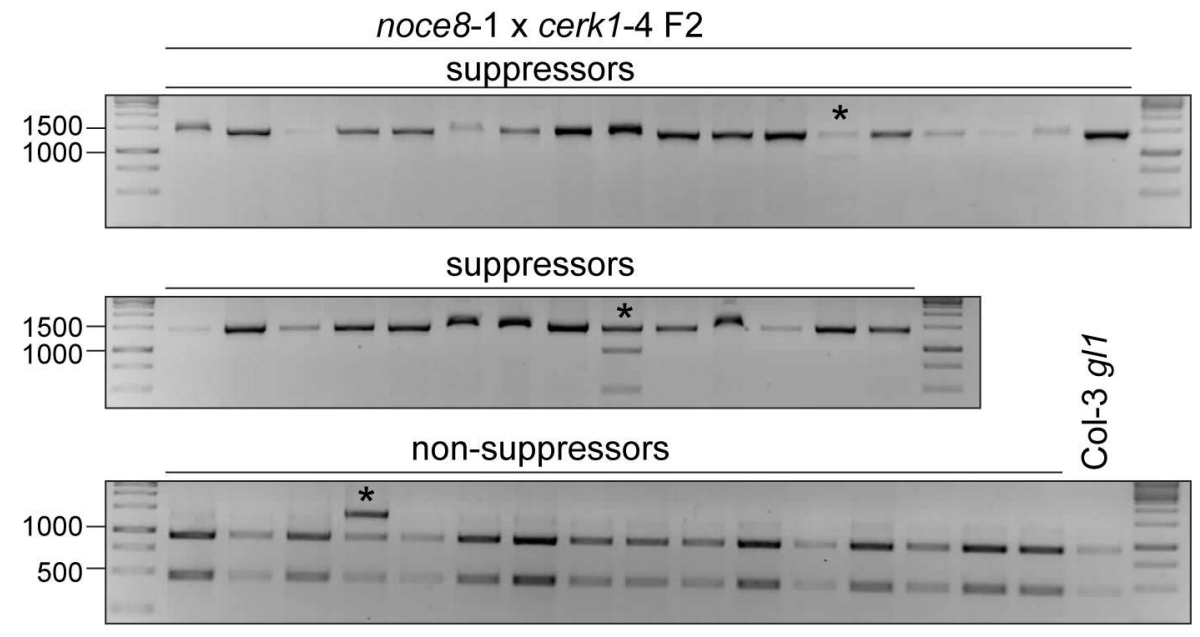

Figure S8: Genotyping of crk43-2 in plants with suppressor and non-suppressor phenotypes.

The crk43-2 mutation was genotyped by PCR using oCT86 + oCT90 and restriction digest of the PCR product with PfeI. PfeI cuts the 1506 bp PCR fragment twice in CRK43 (934 bp, 478 bp, 90 bp) but only once in crk7-4 (1412 bp, $90 \mathrm{bp}) .32$ noce8-1 x cerk1-4 F2 plants with suppressor phenotype and 16 noce81 x cerk1-4 F2 plants with non-suppressor phenotype were genotyped. 30/ 32 suppressor plants were homozygous for the crk43-2 mutation and 15/ 16 non-suppressor plants were homozygous for WT CRK43. The asterisks mark heterozygous plants. Col-3 gll was included as control.
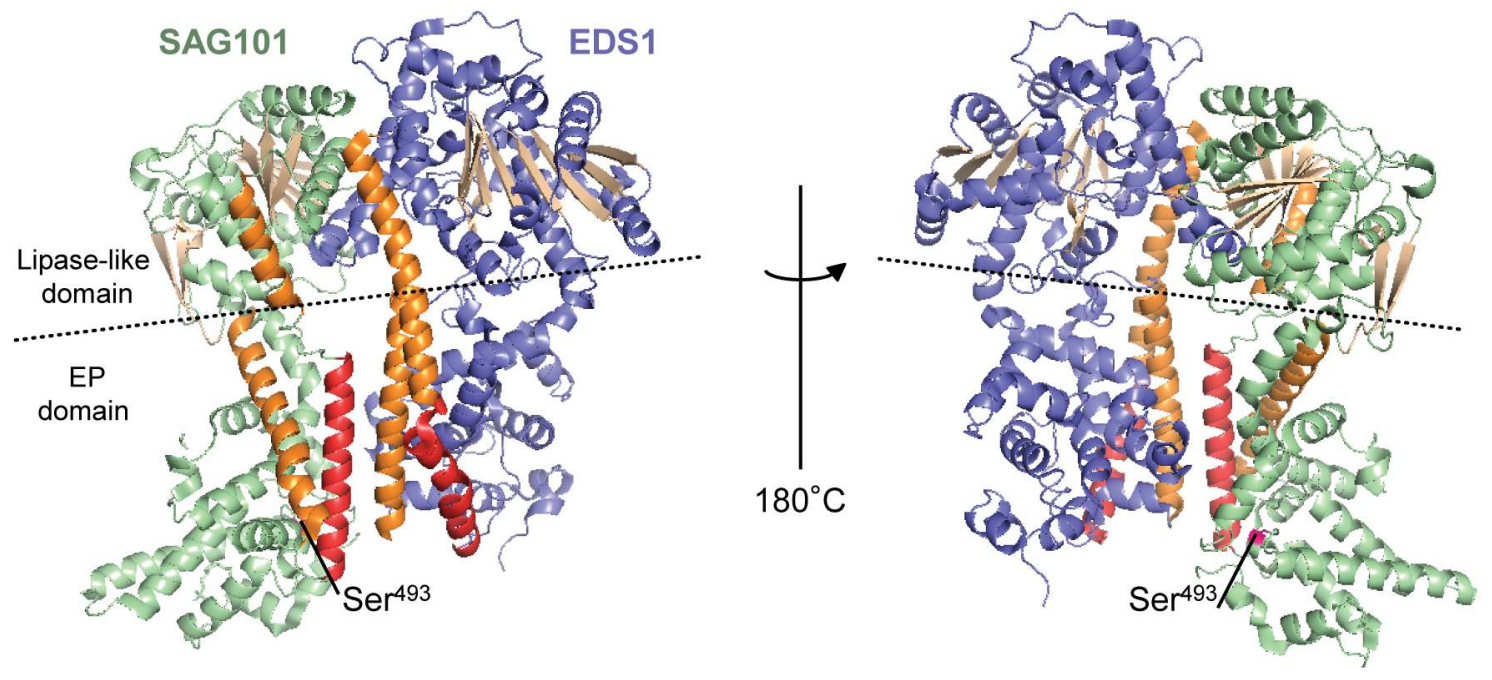

Figure S9: Protein structure of the SAG101-EDS1 heterodimer.

$\alpha$-helices (highlighted red and orange) form a helical groove likely important for ligand interaction. Serine $^{493}\left(\mathrm{Ser}^{493}\right.$ ) in SAG101 that is homologous to serine ${ }^{499}$ in PAD4 is highlighted pink. Structure was adapted and modified from Wagner et al., 2013 using the PyMOL Molecular Graphics System. 
Table S1: Phosphorylation sites in CRK7-Citrine and CRK7-4-Citrine proteins isolated from $N$. benthamiana.

CRK7-Citrine (Cit) and crk7-4-Cit were transiently expressed in N. benthamiana under the native or $35 S$ promotor. Infiltrated leaf areas were harvested 2 dpi and proteins were extracted. An overnight $\alpha$-GFP pull-down was performed, followed by an on-bead tryptic digestion and a C18-purification. The samples were used for LC-MS with the Orbitrap Q Exactive HF mass analyzer. 10 phosphopeptides were chosen for targeted selected ion monitoring (tSIM) analysis. Amino acids highlighted in pink belong to the activation loop. JX $=$ Juxtamembrane domain. Lighter colors represent expression from the $p C R K 7$ promotor, darker colors expression from the $p 35 S$ promotor. Results for CRK7-Cit are highlighted in turquoise, results for CRK74 -Cit in orange.

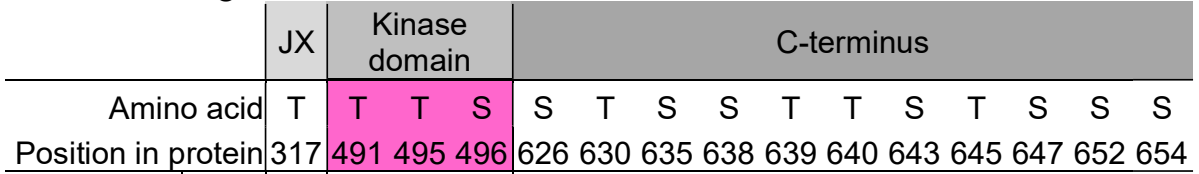

\begin{tabular}{|c|c|c|c|c|c|c|c|c|c|c|c|c|c|c|c|c|}
\hline \multirow{2}{*}{$\begin{array}{l}\text { pCRK7:: } \\
\text { CRK7-Cit }{ }^{A}\end{array}$} & iMS $^{B}$ & -D & & & & & & & - & & & $\bullet$ & & & - & $\bullet$ \\
\hline & tSIMc & 0 & 42 & 282 & 554 & 70 & 23 & 19 & 150 & 33 & 0 & 2441 & 17 & 9 & 169 & 23 \\
\hline \multirow{2}{*}{$\begin{array}{l}\text { pCRK7:: } \\
\text { crk7-4-Cit }\end{array}$} & iMS & & & & & & & & & & & & & & $\bullet$ & \\
\hline & tSIM & 0 & 0 & 0 & 0 & 41 & 0 & 0 & 172 & 16 & 0 & 3775 & 90 & 0 & 230 & 41 \\
\hline \multirow{2}{*}{$\begin{array}{l}\text { p35S:: } \\
\text { CRK7-Cit }\end{array}$} & iMS & & & & $\bullet$ & & & & & & & $\bullet$ & & & & \\
\hline & tSIM & 0 & 0 & 23 & 163 & 0 & 0 & 0 & 70 & 12 & 12 & 4192 & & 0 & 81 & 0 \\
\hline \multirow{2}{*}{$\begin{array}{l}\text { p35S:: } \\
\text { crk7-4-Cit }\end{array}$} & iMS & & & & & & & & & & & $\bullet$ & & & & \\
\hline & tSIM & 0 & 0 & 0 & 0 & 13 & 0 & 0 & 13 & 0 & 0 & 4363 & & 0 & 13 & 0 \\
\hline
\end{tabular}

${ }^{\mathrm{A}} \mathrm{Cit}=$ Citrine

${ }^{B}$ Identified in the initial LC-MS run.

${ }^{\mathrm{C}}$ Phosphorylation sites identified in targeted selected ion monitoring (tSIM) runs.

${ }^{D}$ Dots represent phosphorylation sites identified in the initial LC-MS run (iMS). 
Table S2: Phosphorylation sites in CRK7-Citrine and CRK7-4-Citrine proteins isolated from Arabidopsis.

p35S::CRK7-Citrine (Cit) and p35S::crk7-4-Cit were stably transformed into cerkl-4 plants. Proteins were extracted from six-week old T2 plants. An overnight $\alpha$-GFP pull-down was performed, followed by an on-bead tryptic digestion and a C18-purification. The samples were used for LCMS with the Orbitrap Q Exactive HF mass analyzer. 20 phosphopeptides were chosen for targeted selected ion monitoring (tSIM) analysis. Amino acids highlighted in pink belong to the activation loop, amino acids highlighted in purple to the P+1 loop. Turquoise colors highlight CRK7-Cit PSMs, orange colors CRK 7-4-Cit PSMs.

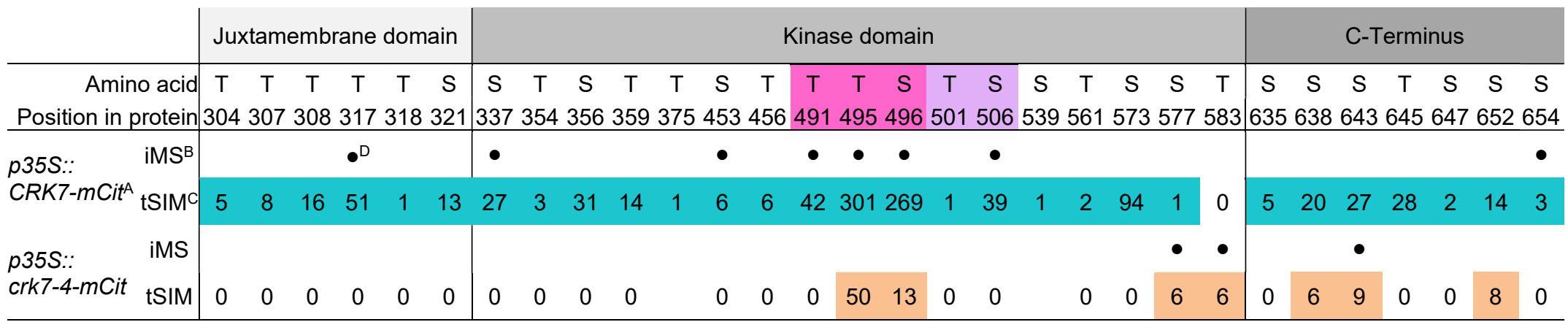

${ }^{\mathrm{A}} \mathrm{Cit}=$ Citrine

B Identified in the initial LC-MS run.

${ }^{C}$ Phosphorylation sites identified in targeted selected ion monitoring (tSIM) runs

D Dots represent phosphorylation sites identified in the initial LC-MS run (iMS). 


\section{List of figures}

Figure 1: Schematic overview of the two-layered plant immune system.

Figure 2: Domain compositions and protein structures of DUF26 domain containing proteins. . 18

Figure 3: Phylogenetic tree of all CRKs and overview of immunity-related CRK gene expression and mutant phenotypes.

Figure 4: E. cruciferarum infected noce9-1 x cerkl-4 F2 plants show a semi-dominant suppressor phenotype. 77

Figure 5: The suppressor mutation in noce $9-1$ is located on the lower arm of chromosome four.

Figure 6: The $c r k 7-4$ mutation identified in the suppressor screen. 81

Figure 7: A mutation in $C R K 7$ suppresses increased powdery mildew induced $P R I$ gene expression in cerk1-4. 83

Figure 8: Characterization of the $c r k 7-5$ T-DNA insertion line used in this study. 85

Figure 9: cerk1-4 crk7-4 and cerkl-4 crk7-5 show semi-dominant phenotypes upon $E$. cruciferarum infection.

Figure 10: The crk7-6 mutation identified in the cerkl-4 suppressor screen shows a semidominant phenotype.

Figure 11: The $c r k 7-7$ and $c r k 7-8$ knockout mutants generated with the CRISPR/ Cas9 system in this work.

Figure 12: cerk1-4 crk7-7 shows a semi-dominant suppressor phenotype upon infection with $E$. cruciferarum. .93

Figure 13: crk7 mutants show WT-like ROS bursts upon flg22 and chitin treatment. .94

Figure 14: crk7 mutants show WT-like MAPK activation upon flg22 and chitin treatment........95 Figure 15: WRKY3O and WRKY53 gene expression is not altered in crk7-5 upon PAMP treatment.

Figure 16: Localization of CRK7-Citrine and CRK7-4-Citrine after transient expression in $N$. benthamiana......

Figure 17: Localization of CRK7-Citrine and CRK7-4-Citrine in cerk1-4.

Figure 18: Localization of CRK7-mCitrine and CRK7-4-mCitrine transiently expressed in $N$. benthamiana...

Figure 19: $C R K 7-\mathrm{mCit}$ and $c r k 7-4-\mathrm{mCit}$ co-infiltrated with cellular markers. 108

Figure 20: Localization of CRK7-mCitrine and CRK7-4-mCitrine in Arabidopsis.

Figure 21: Tagged CRK7 proteins show a high molecular weight band in Western Blot analysis. 
Figure 22: CRK7 is able to form homo- or multimers whereas CRK7-4 is not.

Figure 23: CRK7 shows higher levels of phosphorylation in the activation and $\mathrm{P}+1$ loop than CRK7-4.

Figure 24: Phosphorylation sites identified in CRK7 and CRK7-4.

Figure 25: CRK7-4 interacts with ER chaperones in N. benthamiana. 120

Figure 26: $\mathrm{CRK} 7$ is an active kinase whereas $\mathrm{CRK} 7_{\mathrm{AL}+\mathrm{Pmut}}$ is kinase dead. 122

Figure 27: E. cruciferarum infected noce8-1 x cerk1-4 F2 plants show a semi-dominant suppressor phenotype.

Figure 28: The suppressor mutation in noce8-1 is located on the lower arm of chromosome one.

Figure 29: The crk43-2 mutation identified in the cerk1-4 suppressor screen. 128

Figure 30: A mutation in CRK43 suppresses increased powdery mildew induced $P R I$ gene expression in cerk1-4

Figure 31: Characterization of the crk43-1 and crk43-3 T-DNA insertion lines used in this study.

Figure 32: cerkl-4 crk43-2 and cerkl-4 crk43-3 show semi-dominant cerkl-4 suppressor phenotypes upon E. cruciferarum infection

Figure 33: Expression of CRK43 and CRK43-mCitrine triggers cell death in uninfected cerk1-4 crk43-2 plants.

Figure 34: CRK43 and CRK43-mCitrine expression in cerk1-4 crk43-2 re-establishes the cerk1-4 cell death. 135

Figure 35: crk43 mutants show a WT-like CERK1 band shift upon chitin treatment. 136

Figure 36: ROS burst upon chitin and flg22 treatment is not altered in crk43 mutant lines....... 137

Figure 37: crk43 mutants show WT-like MAPK activation upon flg22 and chitin treatment.... 138

Figure 38: CRK43-mCitrine co-infiltrated with cellular markers in $N$. benthamiana ................. 140

Figure 39: CRK43-mCitrine localization in cerk1-4 crk43-2 T1 plants. 142

Figure 40: E. cruciferarum infected noce12-23 x cerk1-4 F2 plants show a recessive phenotype. 143

Figure 41: The suppressor mutation in noce12-23 is located on the lower arm of chromosome three. 144

Figure 42: The pad4-14 mutation identified in the cerk1-4 suppressor screen. 146

Figure 43: Potential roles of CRK7 and CRK43 in cerk1-4 cell death induction. 166 


\section{List of tables}

Table 1: Arabidopsis thaliana single T-DNA mutant lines used in this work.............................32

Table 2: Arabidopsis thaliana single EMS mutant lines used in this work. ................................32

Table 3: Arabidopsis thaliana double mutant lines isolated/ generated in this work. ..................33

Table 4: Transgenic Arabidopsis thaliana lines generated in this work. .....................................33

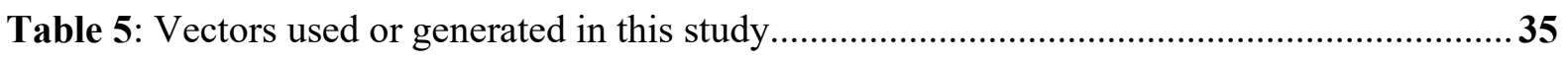

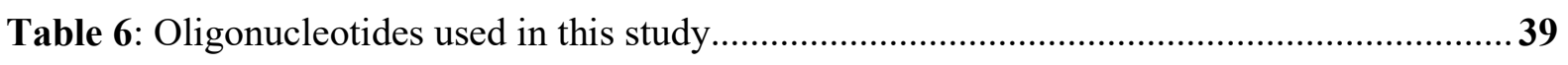

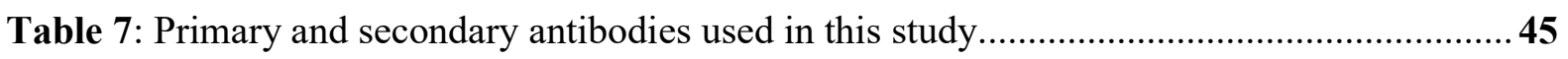

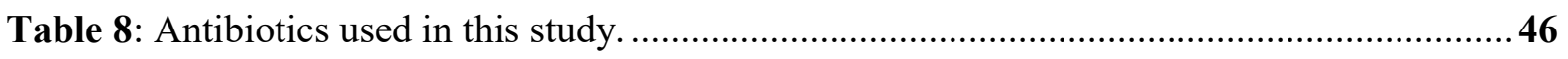

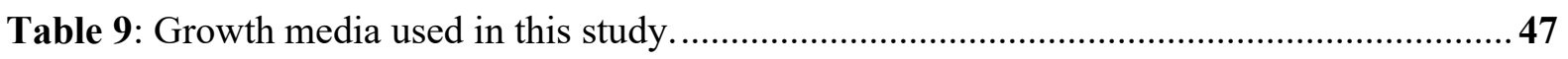

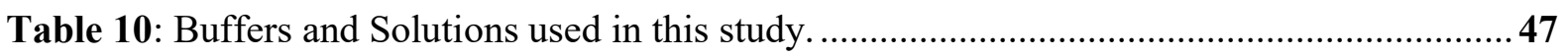

Table 11: Parameters used for detection of the different fluorophores. ......................................56

Table 12: Composition of mixtures used for resolving and stacking gel preparation in this study.

Table 13: Suppressor candidates in noce9-1.

Table 14: Overview of the analyzed lines for complementation transformed with untagged $C R K 7$.

Table 15: Overview of analyzed lines for complementation transformed with CRK7-Citrine (Cit) or CRK7-FLAG constructs.

Table 16: Overview of the analyzed cerk $1-4$ plants transformed with untagged $c r k 7-4$.

Table 17: Overview of the analyzed cerk1-4 plants transformed with crk7-4-Citrine(Cit)/ CRK7Cit. 101

Table 18: Suppressor candidates in noce $8-1$.

Table 19: Suppressor candidates in noce12-23. 


\section{List of supplemental figures and tables}

Figure S1: Cysteine residues in DUF26 domains are highly conserved..................................190

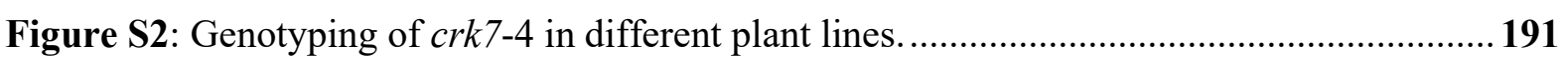

Figure S3: CRK7-mCit and crk7-4-mCit co-infiltrated with cellular markers. .......................... 192

Figure S4: CRK7 shows higher levels of phosphorylation in the activation and $\mathrm{P}+1$ loop than CRK7-4. 193

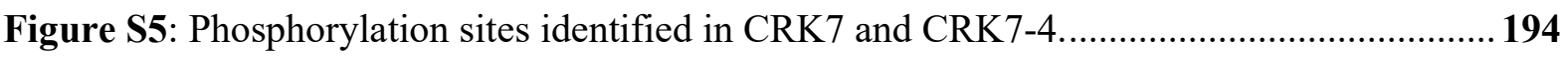

Figure S6: CRK7-4 interacts with ER chaperones in Arabidopsis. ............................................ 195

Figure S7: CRK7 is an active kinase whereas CRK $7 \mathrm{AL}+\mathrm{Pmut}$ is kinase dead. ...............................196

Figure S8: Genotyping of crk43-2 in plants with suppressor and non-suppressor phenotypes. . 197

Figure S9: Protein structure of the SAG101-EDS1 heterodimer................................................ 197

Table S1: Phosphorylation sites in CRK7-Citrine and CRK7-4-Citrine proteins isolated from N. benthamiana..... 198

Table S2: Phosphorylation sites in CRK7-Citrine and CRK7-4-Citrine proteins isolated from Arabidopsis. 


\section{Danksagung}

An dieser Stelle möchte ich mich bei allen Personen bedanken, die mich während meiner Promotion unterstützt und begleitet und somit erheblich zum Gelingen dieser Arbeit beigetragen haben.

Zuerst möchte ich mich bei Prof. Dr. Volker Lipka dafür bedanken, dass er mir die Möglichkeit gegeben hat, an diesem spannenden Thema zu arbeiten. Außerdem danke ich ihm für den Input in vielen Diskussionen und Meetings, der das Projekt vorangebracht hat und für seine Begeisterung. Den Moment, als bei meinem ersten Mapping „CRK7“ in der Kandidatenliste aufgetaucht ist, werde ich wohl - auch dank ihm und seiner Begeisterung - nie vergessen. Vielen Dank außerdem für das Vertrauen und die Unterstützung, die mir entgegengebracht wurden. Desweiteren danke ich ihm für seine Funktion als Erstprüfer und für die Begutachtung dieser Arbeit.

Ein großes Dankeschön geht auch an Dr. Elena Petutschnig für die Unterstützung während der gesamten Zeit meiner Promotion. Ich danke ihr von Herzen, dass ihre Tür immer offen stand und für die vielen Stunden, in denen wir mein Projekt diskutiert, umstrukturiert und geplant haben. Außerdem danke ich ihr, dass sie mich manchmal von Experimenten überzeugt hat, denen ich anfangs skeptisch gegenüber stand, die sich dann aber doch meist als sinnvoll erwiesen haben. Des Weiteren danke ich Elena für ihre geduldige Unterstützung vor allem am Mikroskop und bei den Kinase-Assays. Auch die vielen schönen Gespräche, die oft über den Laboralltag hinaus gingen, habe ich sehr genossen. Ein riesiges Dankeschön geht außerdem an Elena für ihren Input und die Diskussionen in diversen Zoom-Meetings, die mich in der Schreibphase immer sehr vorangebracht haben und natürlich für die Korrektur dieser Arbeit und die dazugehörigen Nachtschichten.

Ein weiteres großes Dankeschön geht an PD Dr. Marcel Wiermer für die kurzfristige Übernahme des Korreferats sowie für seinen Input in den Progress Report im Laufe meiner Promotion.

Desweiteren danke ich PD Dr. Thomas Teichmann für seine Unterstützung während der Promotion und seine hilfbereite Art. Besonders möchte ich ihm dafür danken, dass er sich stets um die „Cruci-Schränke“ gekümmert hat, wenn diese mal wieder Probleme bereitet haben und dafür, dass seine Tür immer offen stand.

Prof. Dr. Kai Heimel, Prof. Dr. Christiane Gatz und Prof. Dr. Andrea Polle danke ich herzlich für die Bereitschaft, Teil meiner Prüfungskommission zu sein.

Ein großes Dankschön geht an Lena - mit einer Freundin wie dir Seite an Seite durch die Doktorandenzeit gehen zu dürfen, war ein riesiges Geschenk und ich hätte mir niemand besseren auf dem Büroplatz neben mir wünschen können. Danke für die wunderschöne gemeinsame Zeit, den stetigen Austausch von privaten und laborinternen Neuigkeiten sowie MarburgErinnerungen, die dazugehörigen Lachanfälle und das gelegentliche gemeinsame Augenverdrehen. Danke fürs Zuhören und dein Verständnis. Egal, wo ich als nächstes arbeite - du wirst mir fehlen! Danke auch für deine Beratung in allen Labor-internen Fragen, hier ist vor allem die Unterstützung bei den Proteomics-Experimenten zu nennen. Ohne deine Hilfe und dein fantastisches Protokoll hätte ich vermutlich nicht ein einziges Replikat zu Ende gebracht.

Großer Dank gebührt auch Sabine und Ludmilla, ihr seid mit der Zeit nicht nur so etwas wie meine „Labor-Muttis“, sondern auch richtig gute Freundinnen geworden und habt viel dazu beigetragen, dass ich mich im Labor so wohl gefühlt habe. Ich habe den Austausch mit euch immer sehr genossen. Wir haben viel zusammen gelacht, aber auch aus den unterschiedlichsten Gründen zusammen geweint. Danke für euer Verständnis, eure Umarmungen und die liebevolle 
Atmosphäre, die ihr geschaffen habt. Es war mir ein Privileg zusammen mit euch auf der „Straße der Besten" arbeiten zu dürfen ;). Darüber hinaus danke ich euch von Herzen für all die kleinen und großen praktischen Aufgaben, die ihr für mich im Laboralltag übernommen habt und für das offene Ohr bei allen „Wo-finde-ich-was“ und „Wie-mache-ich-das“ Fragen. Danke auch, dass ihr euch immer so fleißig um das Auffüllen der Pipettenboxen, sowie oft um einen großen Teil des Gieß- und Spüldienstes gekümmert habt.

An Gaby und Melanie geht ein herzliches Dankeschön für alle Bestellungen, eure Hilfbereitschaft, die Übernahme vieler Gießdienste und für die Beantwortung ungezählter Fragen.

Ein großes Dankeschön geht auch an Ronja, du fehlst hier immer noch! Danke für die tolle Zeit, die wir gemeinsam im Labor, im Büro und in den Mittagspausen verbracht haben, für deine riesige Hilfsbereitschaft in absolut allen Dingen, deine Farbberatung bei Powerpoint Folien und dein Seele-streichelndes „Gut gemacht!“.

Moreover, I would like to thank Andrea for the short but great time we had together in the lab. I really enjoyed all our conversations that developed often later in the evening and kept us staying longer in the lab then planned. Thanks for being incredible friendly and helpful all the time and thanks for your emotional support! I was and will always be happy to answer your questions ;).

An Leon geht ein herzliches Dankeschön für die vielen unermütlichen Stunden des Siebens, Fotographieren, Erntens und Mörserns und die vielen Experimente, mit denen du das Projekt während deiner Hiwi-, Labrotation und Bachelorarbeitszeit vorangebracht hast. Danke, dass man sich immer auf dich verlassen konnte. Es hat mir wirklich Spaß gemacht, dich zu betreuen oder vielmehr mit dir zusammenzuarbeiten und ich habe mich immer gefreut, wenn du da warst.

Außerdem danke ich meinen Mit-Doktoranden Sina, Julia, Denise, Mo, Mascha, Leonie, Daniel und Mohammed sowie der Masterandin Josi für die Hilfbereitschaft, den Erfahrungsaustausch, die vielen schönen Gespräche, die sich glücklicherweise nicht immer nur um die Arbeit drehten, für aufmunternde Worte, wo sie nötig waren und das Teilen von Büroschubladeninhalten voranging kakaohaltiger Art. Danke auch für die schönen Unternehmungen außerhalb des Labors. Ein großer zusätzlicher Dank geht außerdem an Denise für den Austausch, den Zusammenhalt und die Unterstützung während der Schreibphase - das war Gold wert!

Ein weiteres großes Dankeschön geht an Feli und Susanne, ohne euch wäre unsere Arbeit so gar nicht möglich. Danke, dass ihr uns Woche für Woche mit frischen Erdtöpfen versorgt, saubere Schalen und Hauben bereitstellt; danke fürs Pikieren von tausenden Pflanzen und fürs Sieben von hunderten Samenpäckchen! Es war immer schön, bei euch vorbei zu kommen und einen Schwatz zu halten.

Feli, dir danke ich darüber hinaus für die Unterstützung und den Zusammenhalt in der schweren Zeit des Verlusts. Danke für deinen Trost, dein offenes Ohr, dein Verständnis, deine Umarmungen und das gemeinsame Trauern.

Ein weiterer lieber Dank geht an Super-Sekretärin Anja, für die Beratung und Unterstützung in allen organisatorischen und so manchen emotionalen Dingen, deine Hilfsbereitschaft sowie die vielen schönen Gespräche und lustigen Aktionen innerhalb und außerhalb des Labors. Vor allem aber danke ich dir, dass der Arbeitstag dank dir oft mit einer Umarmung angefangen hat.

Ein herzliches Dankeschön geht an Tobi, dafür, dass du wirklich alles reparieren kannst und auch immer die Zeit gefunden hast, eine notwendige Reparatur dazwischen zu schieben! 
Außerdem danke ich Lena, Ronja, Anja und Tobi, aber auch allen anderen, die sich mal zu uns gesellt haben, für die vielen schönen Mittagspausen und die durchaus häufiger erheiternden Gespräche.

Den Teammitgliedern der drei Great-Barrier Runs - allen voran dem harten Kern Thomas, Anja und Mo - danke ich für drei wunderschöne, lustige und anstrengende Tage (und den dazugehörigen tagelangen Muskelkater danach). Unser jährliches „Matsch-Fest“ wird mir fehlen.

Letzendlich danke ich dem gesamten Lipka-lab mit allen ehemaligen und jetzigen Mitgliedern dafür, dass mir die Arbeit trotz allen Herausforderungen letztenlich und zum großen Teil auch dank euch Spaß gemacht hat. Danke für die familiäre Atmosphäre und die tollen gemeinsamen Unternehmungen. Ich werde euch vermissen!

Außerhalb des Labors geht zuerst einmal ein riesiges Dankeschön an Alex, den besten Mann, den ich mir an meiner Seite vorstellen kann. Danke für dein Verständnis und deine Geduld, dein Aufmuntern, dein Launen-Ertragen, deine Power-Anti-Frust-Umarmungen, fürs zum Lachen bringen, die Ablenkung vom Schreib-Wahnsinn an meinen Freigang-Samstagen, deine Laborfenster-Besuche und dafür, dass du immer an mich geglaubt hast.

Ein großes Dankeschön geht auch an Franzi - vor allem dafür, dass du so nah an meinem Leben dran bist und ich so nah an deinem Leben sein darf. Danke für all die wundervollen Telefonate, Karten und Gebete, für dein Verständnis und deine Freundschaft, die einfach gut tut; danke für viele gemeinsame Wochenenden mit deiner wundervollen Familie und danke dafür, dass ich bei euch zu Hause sein darf.

Auch einer Reihe von anderen Lieblingsmenschen möchte ich für viele schöne Wochenenden und Treffen, Telefonate, Nachrichten, Karten und Durchhalte-Päckchen danken: Julia und Simone danke für eure beständige Freundschaft, die schon mehr als unser halbes Leben besteht; Rebecca - danke fürs Leben, Glauben und Kreativität teilen; Judith, Jana, Kristin und Simon - danke für die tollen Reisecrew-Wochenenden und Franzi - danke, dass du immer meine Lieblingsmitbewohnerin bleiben wirst, egal wo wir wohnen.

Ein liebes Dankeschön geht außerdem an meine Schwester Mareike und meinen Petter Markus fürs Zuhören und hinter mir stehen und an meinen „Mr. Neffe“ Ole, der einfach ein Goldschatz ist.

Zuletzt möchte ich meinen wundervollen Eltern danken, die diese Zeilen nicht mehr selbst lesen können. Danke für alles, was ihr mir in der viel zu kurzen Zeit mitgegeben habt. Ich empfinde es als absolutes Privileg, Eltern wie euch gehabt zu haben und alle Erinnerungen an euch trage ich als großen Schatz in meinem Herzen.

Papa, du hast meinen Start in die Doktorandenzeit miterlebt und ich hätte niemals damit gerechnet, dass du „mein Buch“, wie du es immer genannt hast, nie selbst in den Händen halten wirst. „Mein Buch“ ist jetzt geschrieben. 